\title{
WestVirginiaUniversity
}

THE RESEARCH REPOSITORY @ WVU

Graduate Theses, Dissertations, and Problem Reports

2018

\section{(De)Homogenizing Diaspora: An Analysis Of Indian Tamil Identities In The Us}

Christabel Devadoss

Follow this and additional works at: https://researchrepository.wvu.edu/etd

\section{Recommended Citation}

Devadoss, Christabel, "(De)Homogenizing Diaspora: An Analysis Of Indian Tamil Identities In The Us" (2018). Graduate Theses, Dissertations, and Problem Reports. 7173.

https://researchrepository.wvu.edu/etd/7173

This Dissertation is protected by copyright and/or related rights. It has been brought to you by the The Research Repository @ WVU with permission from the rights-holder(s). You are free to use this Dissertation in any way that is permitted by the copyright and related rights legislation that applies to your use. For other uses you must obtain permission from the rights-holder(s) directly, unless additional rights are indicated by a Creative Commons license in the record and/ or on the work itself. This Dissertation has been accepted for inclusion in WVU Graduate Theses, Dissertations, and Problem Reports collection by an authorized administrator of The Research Repository @ WVU.

For more information, please contact researchrepository@mail.wvu.edu. 


\author{
Dissertation submitted \\ to the Eberly College of Arts and Sciences \\ at West Virginia University
}

\begin{abstract}
in partial fulfillment of the requirements for the degree of
Doctor of Philosophy in

Geography

Karen Culcasi, Chair

Trevor Harris, PhD

Maria Perez, PhD

Cynthia Gorman, PhD

Ishan Ashutosh, PhD (Indiana University Bloomington)
\end{abstract}

Department of Geology and Geography

Morgantown, West Virginia

2018

Keywords: [India, Diaspora, Indian Diaspora, Tamil Diaspora, Indian Tamil, Tamil, Tamil Nadu, Diaspora, Sound, Memory, Soundscapes, Discrimination, Identity, Identity Politics, Scale, Hybridity, Indian Diaspora Politics]

Copyright 2018 Christabel Devadoss 


\section{ABSTRACT \\ (De)Homogenizing Diaspora: An Analysis of Indian Tamil Identities in the US}

\section{Christabel Devadoss}

The Indian diaspora, like many other minority communities in the US, has been homogenized and misrecognized because of lack of cultural awareness. Indians have recently experienced a rise in hate crimes and violence in a post-9/11 United States. This lack of cultural awareness is a concern for many minorities for multiple reasons, including the intensified fear of being marked as different, being "othered" or misrecognized, and the threat of discrimination and violence. The Indian diaspora is often homogenized as a single entity even in academic studies. Internal politics within Indian communities as well as discrimination within broader US contexts contribute to a very complex, multi-faceted web of identity politics. This dissertation broadly focuses on identity politics of diaspora communities. It provides insights into discrimination and othering in the US by critiquing generalizations and homogenized understandings of the South Asian/Indian diaspora in media and academia. It accomplishes this through an in-depth study of how TamilAmericans experience and perform their complex, scaled, and hybrid identities and how these identities intersect with sound - more specifically, music, accent, language, and environmental sounds. Sound is an often overlooked part of geographic inquiry, but especially important to how people experience identities and discrimination. The goal of this dissertation is to understand more deeply the nuanced ways that diasporic identity is shaped, represented, and lived with respect to sound, scale, and hybridity. This research contributes to diaspora theories by bridging sound and the everyday to broader theoretical concepts like Orientalism, postcolonialism, and critical race theory. This analysis of identity and discrimination brings attention to complex, heterogeneous, and lived experiences of the Indian diaspora, resulting in 15 findings, with a focus in five specific areas. The first set of findings relate to the emergence of hierarchical scalar identities in moments. The second set of findings relate to hybridity and the emergence of postcolonial identities in settler colonial states. The third set of findings link hybridity and scale, demonstrating a complex relationship between these concepts. In some moments, often connected to discrimination, people think about identities as hierarchical. In others, they show that these identities are hybrid, in-between, and challenge remnants of colonial binaries. The fourth set of findings focus on sound and identity politics. These findings suggest that sound is incredibly important to how identity is lived and represented. It also suggests that while discrimination can be based on the visual, people also monitor their own identities and the identities of others through sound. Finally, discrimination is multi-layered as Indian Tamils experience and reproduce discrimination through sound at multiple levels within and outside of Indian communities as well as toward other minorities. These forms of discrimination are often tied to geographic location, with many Indian Tamils experiencing more discrimination in rural, less diverse areas in the US. Through these findings, this 
dissertation contributes to literature on the relationship between identity, hybridity, and scale; identity and sound; and their importance to discrimination and Critical Race Theory. 


\section{Table of Contents}

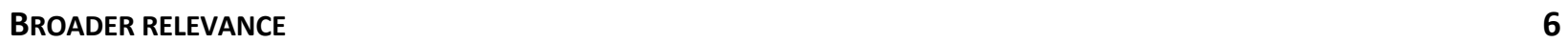

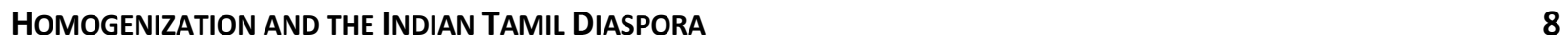

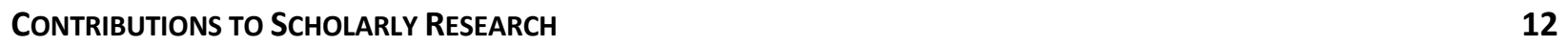

DEHOMOGENIZING IDENTITY THROUGH SCALE AND HYBRIDITY 13

SOUNDS - MUSIC, ACCENT, LANGUAGE, AND ENVIRONMENTAL SOUNDS

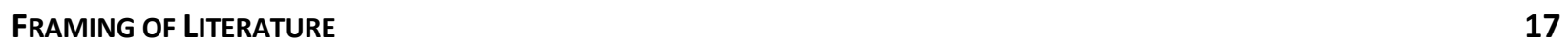

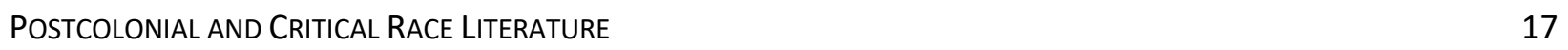

NON-REPRESENTATIONAL THEORY AND SOUNDS 18

$\begin{array}{ll}\text { DIASPORA } & 18\end{array}$

GOAL OF DISSERTATION $\quad 19$

$\begin{array}{ll}\text { STRUCTURE OF DISSERTATION } & 22\end{array}$

\begin{tabular}{lc} 
CHAPTER 2 - LITERATURE OVERVIEW & 24 \\
\hline
\end{tabular}

Postcolonial Scholars, Critical RaCe and Migration Literature, ANd Identity 24

SAID AND THE “OTHER"

HYBRIDITY, IN-BETWEEN, AND MIMICRY

CRItICAL MigRation Literature AND CRItICAL RACE THEORY

NON-REPRESENTATIONAL THEORY AND SOUND $\quad 39$

SOUND AND IDENTITY

DIASPORA - ORIGINS AND TRADITIONAL DEFINITIONS

SECOND-WAVE DIASPORA DEFINITIONS

SCALE AND DIASPORIC IDENTITY

UPCOMING ANALYSIS $\quad 61$

CHAPTER 3: CASE STUDY AND METHODS $\quad 63$

THE INDIAN DIASPORA - COMPLEX AND MULTI-FACETED

INDIAN DIASPORA IN THE US $\quad 65$

INDIAN TAMIL DIASPORA IN THE US

FigURE 1: STATE OF TAMIL NADU $\quad 68$

RESEARCH SITES $\quad 69$

FIGURE 2: STATES OF SOUTH INDIA $\quad 71$

$\begin{array}{ll}\text { METHODS } & 73\end{array}$

ALTERED METHODS

$\begin{array}{ll}\text { DATA, CODING, AND ANALYSIS } & 79\end{array}$

$\begin{array}{lc}\text { POSITIONALITY } & 82\end{array}$

$\begin{array}{lr}\text { ANALYSIS AHEAD } & 87\end{array}$ 
HYBRIDITY AND SCALE

FIGURE 3: SPATIALLY-ORIENTED IDENTITIES REFERENCED BY PARTICIPANTS, DEMONSTRATING RELATIONSHIPS BETWEEN SCALE AND HYBRIDITY.

HYBRIDITY AND SCALE DEBATES

SCALE AND HYBRIDITY EXISTING SIMULTANEOUSLY

VARYING HYBRID AND SCALAR IDENTITIES $\quad 100$

COMPLEX IDENTITIES $\quad 103$

$\begin{array}{ll}\text { TENSIONS BETWEEN IDENTITIES } & 109\end{array}$

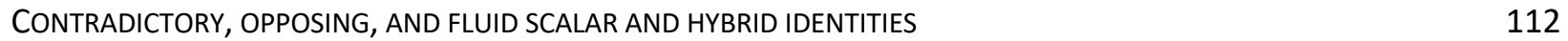

HYBRID AND SCALAR INDIAN VS TAMIL 113

HYBRID AND SCALAR INDIAN VS. TAMIL IN AMERICAN CONTEXT 127

$\begin{array}{lr}\text { IMPLICATIONS } & 140\end{array}$

\begin{tabular}{lc} 
CHAPTER 5: SOUND AND IDENTITY & 142 \\
\hline
\end{tabular}

OVERALL SOUND FOR TAMIL AMERICANS $\quad 143$

SOUND AND TAMIL IDENTITY 146

TABLE 1: SOUNDS AND VARIOUS IDENTITIES 152

FIGURE 5: A VISUAL DEMONSTRATION OF HOW SOUNDS INTERSECT WITH IDENTITIES.

ENVIRONMENTAL SOUNDS $\quad 154$

FOOD AND ENVIRONMENTAL SOUNDS $\quad 163$

MUSIC AND IDENTITY

Figure 7: 2016 CLEVELAND ThyAGARAJA PROGRAM GUIDE WITH MAP OF CLEVELAND, OH AT THE CENTER. SOURCE: 2016

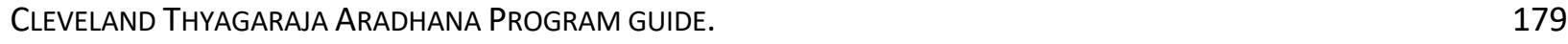

LANGUAGE $\quad 182$

$\begin{array}{ll}\text { LANGUAGE AT EVENTS } & 188\end{array}$

$\begin{array}{lr}\text { ACCENT } & 191\end{array}$

$\begin{array}{lr}\text { SUMMARY } & 197\end{array}$

CHAPTER 6: DISCRIMINATION AND “OTHERING” IN THE US FOR INDIAN TAMILS 199

FIGURE 8: WAYS THAT DISCRIMINATION AND OTHERING INTERSECT WITH SPATIAL/HYBRID IDENTITIES AND SOUND* 204

SOUTH ASIANS AND STEREOTYPES IN THE US $\quad 205$

CONSTRUCTIONS OF “OTHER" $\quad 209$

IMPORTANCE OF GEOGRAPHICAL LOCATION $\quad 212$

RECENT ISLAMOPHOBIA, XENOPHOBIA, AND WHITENESS $\quad 215$

THE RISE OF ISLAMOPHOBIA AND IMPLICATION OF BROWNNESS $\quad 227$

ORIENTALISM AND OTHERING $\quad 233$

WEB OF DISCRIMINATION $\quad 242$

$\begin{array}{ll}\text { CONTEXT AND US DISCRIMINATION } & 248\end{array}$

CHAPTER 7: DISCRIMINATION, “OTHERING,” AND INDIAN IDENTITY 252 
WHITENESS AND MARGINALIZATION OF TAMILS IN THE INDIAN DIASPORA

EMERGENCE OF HIERARCHICAL SCALAR IDENTITIES 296

HYBRIDITY - POSTCOLONIAL IDENTITY 300

THE TENUOUS RELATIONSHIP BETWEEN SCALE AND HYBRIDITY AND IDENTITY 303

SOUND AND IDENTITY POLITICS 304

MULTI-LEVEL DISCRIMINATION, OTHERING, AND IDENTITY 307

FUTURE WORK

DELIMITATIONS AND LIMITATIONS

CONCLUDING THOUGHTS

REFERENCES

APPENDIX A

APPENDIX B

APPENDIX C 
Dedication: I dedicate this to all of my grandparents who are the reason I am here today Dolores Boos, Hannah Christabel Devadoss, Donald Boos, and Sundaram Devadoss 
Acknowledgements: First and foremost, I would like to thank my advisor, Karen Culcasi for 4 years of dedicated support and detailed feedback. I would also like to thank my committee, Dr. Ishan Ashutosh, Dr. Maria Perez, Dr. Trevor Harris, and Dr. Cynthia Gorman for their feedback and guidance throughout the dissertation process as well.

Peer support is incredibly important, so I would like to thank my wonderful support group at WVU - Jothiganesh Shanmugasundaram, Barb MacLennan, Frank LaFone, Hari Hara Sudhan Lakshman, Pragya Srivastava, Joshua Lohnes, David Knieter, Jennifer Smith, Amanda Marple, Fang Fang, Alex Dye, Chris Ramezan, and so many others. I would also like to thank my former Kent State colleagues Samuel Henkin, Gordon Cromley, and Seth Johnson for additional support throughout this process.

I also received teaching evaluations, advice, and feedback during my time as a doctoral student, so I would also like to acknowledge Dr. Jonathan Hall, Dr. Timothy Warner, Dr. Jamie Shinn, and Dr. Brent McCusker for taking time to guide me. I want to also thank Hope Stewart for the administrative assistance during my PhD.

Finally, I am grateful for the support from my family - especially, my life partner, David Murad, my parents Jonathan Devadoss and Donna Devadoss and my brother, Joshua Devadoss. 


\section{Chapter 1 - Introduction}

“...the desire for a reformed, recognizable Other, as a subject of a difference that is almost the same, but not quite." - Homi Bhaba (The Location of culture).

Growing up, I heard a lot of stereotypes about Indians - Indians in India were poor and dirty, but in the US, they were supposed to be smart and wealthy. When some people found out that my father was not a doctor, they would say, "Well, you must be the poor Indians from those untouchable castes." It was clear to me that he was still too far from "almost the same, but not quite." More than once, someone told me that my dad reminded them of one of those cannibals from Indiana Jones and the Temple of Doom, because he was tall unlike most Indians they saw, but also because he didn't say much. He was too quiet, or they couldn't understand his accent. Before 9/11, in our rural Ohio town, my father, brother, and I were the Indians (both seriously and 'jokingly') that lived in "teepees" for those who were unaware that India was a country, or the savages from a place where people "eat with their hands." We were also Apu, the "Indian" character from The Simpsons. People assumed that all Indians were the same and spoke "Hindu" or "Indian." When I said that my dad spoke Tamil, most people did not even know that India had more than one language.

After 9/11, we were the "brown" people and potentially Muslim invaders. Yet, it was in this post-9/11 world that I witnessed my dad seemed to emphasize his Indianness. "I'm 
going to be Indian until I die," he would say. He has lived in the US for more than 35 years, but at times, talked about Indian identity as if it was simple and fixed, emphasizing it at the scale of the nation. However, the way in which he lived and experienced this Indian identity, I realized, was far more complex and hybrid, and changed scales depending on context. After events like 9/11, the election of Donald Trump, or when he was the focus of stereotypes, my father seemed to tie his identity to a broader scale of brownness (South Asian being one ${ }^{1}$ ), to distinguish himself (or reflect the distinction that others made) that he was not white. Simultaneously, I saw that he also emphasized his identity at the national scale of India. I gained the impression that it was important for him to be nationally Indian in political climates that threatened his perceived core identity. To counter this threat and reinforce the national scalar identity of Indian, it seems like my father often falls back on both Hindi and Tamil-language music and movies.

Like other identities, Indian identity can manifest through language, music, and even accent, and my personal experience emphasizes these manifestations. Such sounds can be become a double-edged sword. On one side, sounds reinforce someone's core identity. On the other side, they can be used to mark or differentiate. As many scholars have noted, sounds mark identities as other or non-normative, leading to potential discrimination or othering (Haldrup, Koefoed, \& Simonsen, 2006; Jazeel, 2005; Kanngieser, 2012; S. Sharma, 2006; Simonsen, 2010). In other words, sounds of Tamil or any other Indian language, accent, and/or music can be markers of difference to bring attention to non-whiteness in the US, which could potentially result in stares, racial slurs, threats, police racial profiling,

${ }^{1}$ I develop and elaborate on how brownness is a scale in later chapters. 
or the distinction between "American" and immigrant (Hopkins, Botterill, Sanghera, \& Arshad, 2017).

Sounds do not just differentiate national or supranational identity. They also have the potential to mark regional differences, especially within Indian communities. For example, in situations with other Indians, my father scaled down, emphasizing his regional, Tamil identity. He spoke and grew up with Tamil, not Hindi, the latter of which is the national language of India². I've heard my father describe himself as a Dravidian, which indicates one from South India. ${ }^{3}$ He talks about how Dravidian languages, especially Tamil, are the oldest in the world with the oldest sounds and oldest music. I've seen him struggle to speak Hindi when visiting India and or in crowds where everyone else speaks Hindi. I noted the angst reflected in his comments that some North Indians don't like South Indians or the Tamil language. Yet, he also/simultaneously seems embarrassed by his Tamil accent. He speaks a more Northern, colloquial, informal Tamil that, after living in the US for so long, is not proper, "pure" Tamil.

And while he makes distinctions between his identities, describing them in moments I interpret as both hierarchically scaled (i.e. Indian, Tamil, South Asian), and multi-scaled (Indian and Tamil simultaneously), these identities are also very much hybrid. Occasionally, though he would never admit it, he demonstrates that his identity is both Indian and American. For example, he talks about how Americans can be rigid with time schedules (not being late, etc.), yet has adopted these practices and is incredibly frustrated by the lack of scheduling when he is in India - describing it as chaos. He also enjoys popular

2 Officially, English and Hindi are national languages (Velayutham, 2008).

3 This term is a bit more complicated, but I explain it in more detail later in the dissertation. 
American movies and entertainment, not just Indian entertainment. My dad's identity is also hybrid in a way that he admits, because he always links it back to the shadow of colonization, which created conditions that both binarized and homogenized identities. He describes himself as Tamil, but yet shows that he is not "purely" Tamil. He describes himself as "Indian," but when visiting India, comes across as an NRI (non-resident Indian) someone who has been abroad for a significant amount of time. Living in the US for many years, he was often in a state of being "in-between". He is Tamil, but also listens to Hindi music and watched Hindi-language movies. He is Indian, yet changed his Indian, BritishEnglish over the years to reflect more American English. He connects this purposeful change to distancing himself from British colonialism and also as a way to better fit in the US.

I introduce my dissertation by discussing my father because his expressions of identities challenge the homogeneity of Indian identity. My brief reflection on his life highlights how scales, sounds, and discrimination are important components to navigating these identities. My father's life and complex identity has fueled my intellectual curiosities to critique the ways that popular culture, governments, and scholars discuss Indian diaspora identity - and particularly to examine how scale, hybridity, and multiple senses, specifically sound, all contribute to these diaspora identities.

Over the course of my data collection, I interviewed many participants who described similar experiences to that of my father in how they negotiated their identities. They also described experiences that differed from his in complex and variegated ways. It is through their stories and the concepts of hybridity, scale, and sound that I examine complex, varied Indian Tamil identities. 
Scale and hybridity have a complex, tenuous relationship but both are intricately weaved into identity. Those I interviewed expressed different identities dependent upon their position in a complex social hierarchy shaped by both a legacy of colonialism and the homogenization of Indian identities. These scalar hierarchies are reinforced by governments, academics, and popular media as static - either Indian, American, or Tamil. But these identities are hybrid in that they are not binary or static. They are often fluid and in-between. They are also hybrid because people of postcolonial descent often have to navigate colonial binaries (colonized and colonizer, us vs them, Tamil and Indian, Indian and European).

I describe this process of hybridity and scale in more detail throughout the dissertation, but a few brief definitions are valuable from the outset. Scale demonstrates how identities, often homogenized, are heterogenous but at times solidify. Hybridity, meanwhile, shows the complex, overlapping, and hyphenated nature of these heterogenous identities. Hybridity is a complex term that has a plethora of meanings. These meanings range from the blurring of identities (places or spaces), being in-between two identities (places or spaces), to a state of being that resulted from the aftereffects of colonialism (Anzaldúa, 1987; Gilmartin \& Berg, 2007; L. M. Harris, 2014; Jazeel, 2006; Loomba, 1998; Sharp, 2011; J.D. Sidaway, 2000).

To illuminate the importance of scale and hybridity to identities in a visual-heavy discipline, sound is one unique approach. It also demonstrates nuance in how people construct identities when facing discrimination or othering. Sound - specifically music, accent, language, and at times, environmental sounds - untangle everyday experiences of identity and experiences of discrimination. Sounds can be markers that people use to 
distinguish, discriminate, or monitor their identities and the identities of others. I analyze sound's role in identity in two ways: first, how people understand their identities through sound and second, how sound contributes to "othering", differentiation, or discrimination of specific identities.

My goal is to challenge homogenized identity and, in the process, argue that sound, scale, and hybridity lends significant insights to complex processes of identity, othering and discrimination. More specifically, my goal is to acknowledge and demonstrate the significance of recognizing nuances of identity and discrimination within the Indian Tamil diaspora.

\section{Broader relevance}

Generally, minorities have the intensified fear of being "othered," while also facing the threat of overt, structural discrimination and hate crimes (Chou \& Feagin, 2015; Hopkins et al., 2017). Among minorities, the Indian diaspora has encountered a great deal of homogenization and stereotyping that contribute to instances of discrimination and othering $^{4}$ - defined simply for this introduction as being marked as different or marked as not belonging (Hopkins et al., 2017; Velayutham, 2008b). This othering of Indian Americans has coincided with a long history of violence, including from the early $1900 \mathrm{~s}$ and prior to the 1965 immigration act that opened migration from Asia ${ }^{5}$ (Bhardwaj \& Rao, 1990; Safran, Kumar Sahoo, \& Lal, 2008). For example, in the 1910s, the Asiatic Exclusion

\footnotetext{
${ }^{4}$ Othering has a much more complex background linked to colonialism and Orientalism which I describe in greater detail in Chapter 2.

${ }^{5}$ Shifting the violence to other minorities in Central and South America - I discuss this a bit later in the dissertation footnotes, but I want to recognize this now.
} 
League and the American Federation of Labor described Sikhs as a 'Tide of Turbans', 'ragheads' and even a distinct 'menace'", excluding them from work and US society (Bhardwaj and Rao, 1990, p. 198). But even more recently, Indians have experienced a rise in hate crimes (Kishi, 2017; PRC, 2014; Safran et al., 2008; Sridaran, Raghunathan, \& Trivedi, 2017). The shooting of two Indian Americans in Kansas in 2017, the Wisconsin Sikh temple massacre in 2012, racist reactions to an Indian-American winning the Miss America pageant in 2014, police brutality that paralyzed an elderly Indian man in 2015, are a few of the many incidents that demonstrate recent othering, discrimination, and violence against Indian Americans (CNN, 2013; Sridaran et al., 2017; Talbot, 2015).

One irony is that while many Indians experience discrimination and violence in the US, as a group, they are perceived to be privileged, a model minority, and exempt from the racial and ethnic violence directed at Native Americans and African Americans (Chou \& Feagin, 2015; Dave, 2013). Part of the reason for this perceived privilege is because they have the highest median income of any 'ethnic group'6 (Chou \& Feagin, 2015; Skop, 2012). Asian Americans, and especially Indian Americans, have often been touted as the secret to "economic success" and used as a tool to show other "minorities" that they too can succeed and attain positions of many white Americans (Chou \& Feagin, 2015). However, while some Indians fit the economic "model minority" stereotype, they nevertheless have been affected by Islamophobia, anti-immigrant sentiments, the rise of white supremacy, and homogenization (Hopkins et al., 2017; Iyer, 2014; Modi, 2018; Sridaran et al., 2017). Thus, the most striking irony is that because Indian Americans are often associated with

\footnotetext{
${ }^{6}$ I use this term (used by the authors cited) to make a point, but recognize that the term
} ethnic is somewhat problematic. 
stereotypes like the model minority in popular narratives, many view themselves as closely connected to whiteness (Bhatia, 2007; Safran et al., 2008) - a point I develop later in the dissertation.

Ultimately, the homogenization or stereotyping of Indian communities has potentially violent consequences. Bringing attention to heterogeneity not only combats these potential consequences but illuminates the diverse ways in how people live and experience identity. Scale and hybridity, I argue, are also important to these complex identities. Analyzing sound, more specifically, music, accent, language, and environmental sounds, reveals nuance, as well as documenting how sounds contribute to othering and discrimination. I will delve into these concepts below, but first I introduce the Indian Tamil diaspora and address more specifically, the various ways in which Indian identities are homogenized. I point out this homogenization to further establish the relevance of my study.

\section{Homogenization and the Indian Tamil Diaspora}

The Indian Tamil diaspora is a subgroup of the Indian diaspora, often describing those with connections to the Indian state of Tamil Nadu. The Indian Tamil diaspora is a prime case study to discuss nuance in diasporic identity, precisely because it has its own unique history, cultures, and traditions (including its own music, accent, and language) that differ from what is broadly understood as Indian (Devadoss, 2017; Krishnan, 2008; Velayutham, 2008b). The Indian diaspora is heterogeneous, with many regional affiliations and diverse identities, but such diversity is often masked in academic, government, and popular media representations of Indian communities (Bhatia, 2007; Bose, 2018; 
Velayutham, 2008). Thus, examining the Indian Tamil diaspora specifically challenges such representations (Velayutham, 2008).

The Indian diaspora, I argue, is homogenized in five ways. First, it is homogenized by the US government. For example, the US Census Bureau classifies all Indians as "Asian Indian" in Census data - data used and standardized in academic and governmental research. This single category limits researchers from exploring the diversity of Indian diaspora and combating stereotypes of the Indian diaspora. As Bose (2018, p. 262) describes of Census data, it "is a poor way of aligning the various parts of the diasporic identity - region language, ethnicity, and culture being a few of these." In other words, it homogenizes.

Second, Indians are homogenized by US popular culture. Characters like Apu from the Simpsons (Indians as convenience store owners) or nerdy, science or tech people dominate US popular culture, especially in films and TV shows (Dave 2013). Popular Indian American comedians like Aziz Ansari and Hari Kondabolu have recently critiqued such Hollywood stereotypes. Ansari suggests that Indians are often typecast to play taxi drivers, gas station owners, and have a standardized "Indian" accent (Ansari and Yang, 2015). As Dave (2013, p. 55) states, "this lack of understanding ... signals how Indian Americans are lumped into one group regardless of significant linguistic, religious, political, and cultural histories." Ultimately, these representations contribute to a simple, stereotyped narrative of Indians in the US.

Third, Indians are homogenized in academic studies, which often focus on North Indian groups, Bollywood films, upper class Hindu narratives, or, given the Census data cited above, "Asian Indians". Academic studies on the diaspora commonly focus on North 
Western and North Central Indian states (Krishnan, 2008; Velayutham, 2008b). These studies also focus on ethnic communities in the Punjabi, Gujarati, or other Northern landscapes. Finally, these studies often examine Hindu landscapes of Indian communities, perpetuating the narrative that all Indians are Hindu and are not culturally, religiously, or linguistically diverse (Bhatia, 2007; Radhakrishnan, 2003; Ramaswamy, 2009).

Fourth, Bollywood films recreate many of these homogenized stereotypes. Films marketed directly at the Indian diaspora (often the US or UK), such as Namastey London, Kabhi Khushi Kabhi Gham, Dilwale Dulhaniya Le Jayenge, New York, generally portray Indians as North Indian Hindi speakers. Heroes and heroines often hail from North Western or North Central roots, especially. Meanwhile, peripheral groups like Tamils are typically portrayed as backward or unsophisticated (Ganti, 2013; Srivastava, 2013; Velayutham, 2008a). For example, the 2013 film Chennai Express presents the Tamil language as harsh, rough, and loud compared to the softer, more gentle tones of Hindi. The film also portrays Tamils as uneducated thugs with very dark skin compared to lighterskinned North Indian protagonists.

Fifth, the Indian government homogenizes the Indian diaspora through official documents. For example, many within the Indian diaspora are referred to as NRIs (nonresident Indians), a classification that ignores the fact that Indians have different citizenship policies and privileges. This single NRI category could potentially emphasize Indian as Hindu, Hindi-speaking, and North Indian and ignore states like Tamil Nadu (Devadas, Vijay and Velayutham, 2008). The Indian Government's Report of the High Level Committee on the Indian Diaspora (RHLCID) (2001) homogenizes the Indian diaspora as a singular category. It recognizes two waves of diaspora - the initial wave of forced 
migration from colonial rule and the second wave of voluntary migration - but continues to describe them as a unified diaspora (Dickinson \& Bailey, 2007). The RHLCID (2001) outlines the diaspora based on where the diaspora has immigrated, not from where they have emigrated.

Homogenization is but one of many factors that contributes to discrimination and othering. Yet, it is pervasive. Since homogenization is so ubiquitous, recognizing heterogeneity and nuance are important and as I argue in my dissertation, homogenization and lack of understanding of nuance have contributed to experiences of hate crimes and othering in recent political climates (Dave, 2013; Hopkins et al., 2017).

The shooting of Indian Americans in Kansas in 2017 and the Sikh temple massacre in 2012 have ended with loss of life, but recent blatant targeting of Indian Americans based on homogenized stereotypes is also rising. For example, websites like "Save American IT Jobs" in Columbus, OH made US headlines for targeting people of Indian descent, precisely because its creator emphasized stereotypes suggesting that all Indians are H1-B visa workers taking IT jobs. Though since taken down, the website encouraged non-Indian community members to stalk and target Indian Americans and collect evidence of how they are destroying the Columbus community. Huffington Post India (2017) quoted the website founder's video as the founder reinforced stereotypes: "The Indian crowd has ravished the Midwest. It's crazy. I ask this question -- what happened to all the American people that used to live in this middle, upper-middle class neighborhood. Where does all this money come from?" He then subsequently criticized the H1-B visa program for "destroying American lives", suggesting that Indians and Indian Americans are not really American, even if they have citizenship. This sentiment is furthered by current US government 
narratives. As of April 3, 2017 US Citizenship and Immigration emphasizes "targeted site visits" to detect "fraud" - suggesting that H1-B visa fraud is rampant across the US (USCIS, 2017b).

With the rise of white nationalism in post-Trump election climate, violence on South Asians has already reached unprecedented levels and may continue to rise (Modi, 2018; Sridaran et al., 2017). The broader implications of whiteness in post-Trump election America are important and relevant, but they are also old, pervasive issues entrenched in the US since the founding of the country (Bobo, 2017; Gökarıksel \& Smith, 2016; Morris, 2017; Steinberg et al., 2018), and are reflected in current experiences of Indian Tamils in the US.

Homogenization is but one aspect of discrimination and othering yet it is substantial. Indians face significant challenges with discrimination and othering in the US, often connected to stereotypes and homogenization. Homogenization also masks another important issue - discrimination within Indian communities, especially in the form of what Bhabha (1994) and Fanon (1963) describe as mimicry - simply defined as the tendency for the colonized to identify with colonizer to obtain privileges of the colonizer and/or blend in. Homogenized stereotypes ignore both the external and internal politics with respect to Indian diasporas - something I address in greater detail throughout the dissertation.

\section{Contributions to Scholarly Research}

My dissertation is relevant because of its focus on discrimination in current political climates within the context of the US and the Indian diaspora, but it also builds on and contributes to scholarly literature examining diaspora and identity more broadly. Scholars 
have linked homogenization and stereotypes to colonialism, non-representational theory, Orientalism, and critical race theory (Haldrup et al., 2006; Hopkins et al., 2017; Simonsen, 2010). My dissertation builds on this literature in two significant ways. First, it highlights the complex relationship between scale and hybridity and second, it demonstrates the significant role of sound to identity in these bodies of literature.

\section{Dehomogenizing identity through scale and hybridity}

In order to dismantle homogenization, I examine the nuances of identity through the concepts of scale and hybridity. Scholars have examined hybridity and scale separately but have not sufficiently engaged with them in unison (Bhabha, 2015; Nicley, 2009). The relationship between scale and hybridity is at times tenuous, but as I argue, these concepts are connected and draw attention to the nuances of identity.

Scale is an evolving concept in geography. Traditional scale is hierarchical, implying that scales like global, national, regional, local are fixed in a hierarchy, similar to a ladder with global at the top and local at the bottom (Häkli, 2018; Marston, Woodward, \& Jones, 2009). However, one way that geographers have critiqued this traditional view of scale is to argue that scale is contested, socially constructed, not fixed, emergent, not permanent, not pre-existing, and develops "at the moment" (Ferber \& Harris, 2013, p. 190; Marston, 2000). Even so, scale remains relevant in that it is simultaneously lived and experienced in everyday life. In terms of traditional views of scale, those with regional, local, or nondominant identities mobilize counter-narratives to "challenge(s) the hegemonic identity narratives" of the nation-state scale (Paasi, 2003, p. 476; Johnson and Coleman, 2012). Scholars have used scale to provide insights on how people use non-dominant identities, 
such as regional identity, to actively speak out against dominant, constructed national hegemony (Johnson and Coleman, 2012). Different scales of identities, such as regional or national, while socially constructed, are nevertheless mobilized during specific moments to dismantle national, homogenizing accounts of their complex identities (Culcasi, 2011; Johnson \& Coleman, 2012; Mackinnon, 2011; Paasi, 2003).

Geographers recognize that scales are not static and have thus challenged traditional and hierarchical scales, presenting them rather as hyphenated, multiple, and hybrid. However, 'hybrid scales' is not the same as 'hybridity'. Often, when geographers discuss hybrid scales, they describe the blurring and overlap of scales (Nicely, 2009). Yet, hybridity is not simply the blurring of identities or scales (though this is one part of it) - it is a complex process that incorporates colonial legacy and it points to the "destabilize(ing) (of) the fixity of identities" (Bhabha, 1994; Chacko and Menon, 2013, p. 99). Hybridity elucidates how identities come from multiple sources, and can be blurred and in-between, but most importantly, the concept brings to light that identities are often in flux because of the legacies of colonialism that depict identities as binary and fixed (Bhatia 2007; Bhabha, 2015).

Scholars have criticized the concept of hybridity for erasing uniqueness and minimizing instances where people actively engage with, and become less, hybrid in specific moments (Kompridis, 2005; Schrank, 2007). I argue that this is when scale becomes important. For example, people purposefully identify with regional scales to counter-narrate dominant national scales. In doing so, they momentarily reinforce fixity of regional or national identities, especially when facing the fear of being subsumed by homogeneity. In some instances, as Bhabha (2015) documents, scholars have actually used 
hybridity to homogenize. Nevertheless, the concept still calls attention to the conditions of postcolonial, in-flux identities - important to counteracting the traditional, fixity of hierarchical scale. As further discussed in Chapter 2, hybridity situates post-colonial diasporas and identities that are often in-between, blurred, and multiple (Bhabha, 1994).

Identities are hierarchically scaled, yet simultaneously blurred and hybrid, depending on the moment. These moments are also connected to discrimination and othering in the US. For example, my findings illustrate that in moments where interview participants felt othered or discriminated against by other Americans, many identified much more closely with national or supranational identities like Indian or South Asian. Yet, in moments when they faced discrimination or othering within Indian communities, they identified more closely with regional or local scales like Tamil or their specific villages. Thus, these identities, at times described as fixed, became hybrid and overlapped depending on situation and context. Scale demonstrated nuance yet fixity in identity, all tied to discrimination or othering, while hybridity undercut fixity to show how these identities were theorized as fixed precisely because of both former colonial legacy and broader homogenized narratives in the US.

\section{Sounds - Music, accent, language, and environmental sounds}

For years, sounds have been a relatively overlooked aspect of academic inquiry, especially in geography (Gallagher, Kanngieser, \& Prior, 2016; Revill, 2016). While relatively few scholars in other disciplines have examined sound as part of the urban and natural environment or how sound affects human emotion and preferences (Carles, Barrio, \& De Lucio, 1999; Pijanowski et al., 2011), scholars in geography have traditionally focused 
on visuals - maps, texts, flags, images, landscape, etc. (Kobayashi \& Peake, 2000; Rose, $2003,2016)$. Sparse geographic research on sound has produced studies varying from mapping environmental tones in urban environment within cities, or rural and national park systems, to theorizing how sound fits with community and political spaces (Anderson, Morton, \& Revill, 2005; Revill, 2016; Smith, 1994; 2000).

Studies examining how sound affects stereotyping and generalizations of groups and people (Haldrup et al., 2006; Zeitlyn, 2013), often de-emphasize sound's unique role within a multi-sense framework emphasizing senses like taste and smell. They also focus on othering and discrimination from the perspective of those doing the othering, and these are often white Europeans or Americans. For instance, Haldrup et. al (2006) show how certain communities in the Netherlands other people from the Middle East through food and smells (deeming Middle Eastern food as invasive) or billboards (describing people from the Middle East in a negative light). But, in their analysis, they also touch on how their Dutch study group othering the language and accent of Arabic and musical sounds like the call to prayer. While such analyses with sound provide insights into othering and discrimination (Dave, 2013; Haldrup et al., 2006; Kanngieser, 2012; Simonsen, 2010), my analysis demonstrates sound's precise role from the perspective of those being othered and discriminated against. I argue that sound is an important non-visual marker of difference from various perspectives. I found that many people monitor themselves and others through sound - often accent, but also music and language. 


\section{Framing of Literature}

I draw on multiple bodies of literature - Postcolonial, Critical Race, NonRepresentational, and Diaspora literatures - to examine the relationships between identity politics, diaspora, scale, hybridity and sound. Though they are connected and fluid, for the sake of organization, I have separated them. These items will be discussed in more detail in Chapter 2.

\section{Postcolonial and Critical Race Literature}

Postcolonial literature is seminal to examining diasporas and identity (Bhabha, 1994; Gilroy, 1993). Aspects of identity like hybridity, mimicry, and othering, which are central to my analysis, have been examined and theorized by many postcolonial scholars like Fanon, Bhabha, Gilroy, and Said.

It is crucial to situate the Indian diaspora within the history of colonialism, while recognizing how colonization itself has resituated the very conditions of hybridity, embedded within postcolonial diasporas and communities today. Postcolonial literatures suggest that colonialism has shaped boundaries, borders, identities, and contemporary global political situations (Sparke, 1998; Chakrabarty, 2000). In this, postcolonial literature overlaps with Critical Race Theory (CRT), whose foundational idea is that white bodies and culture often make up the standard norm (Dyer, 1997). Fanon $(1967 ; 1963)$ describes whiteness and constructions of race as a direct result of European colonization of the world. Other CRT theorists have echoed Fanon, arguing that everything is defined in relation to whiteness, a direct result of the historical process of colonization (Dyer, 1997). 
Many scholars have built on this CRT literature to describe present-day colonialism or settler colonialism in current climates (Bonds \& Inwood, 2016; Gregory, 2004).

\section{Non-Representational Theory and Sounds}

Non-representational theory, also referred to as non-representational theories (NRTs), focuses on the everyday, lived experience, which was traditionally overlooked in geography, a discipline that focused heavily on discourse, text, and visuals (Doel, 2010). NRTs provide both methodological and theoretical contributions that emphasize human practice and performance, as well as people's everyday experience, over broader media or textual representations (Doel, 2010).

Non-representational theory and postcolonial theory can complement each other to form a more comprehensive analysis of discrimination and othering. Scholars like Haldrup et. al (2006) and Simonsen (2010) have bridged postcolonial concepts like othering to the multi-sensory everyday experience. NRTs focus on the lived aspects of identity, moving away from visuals to highlight additional senses such as sound. Much of the qualitative geographical work on sound, explored further in this dissertation, has been examined through NRTs: for example, Anderson et. al's (2005) examination of music as sound, Kanngieser's (2012) examination of the tonality of voice, and Revill's (2016) discussion of sound in politics. Thus, my own work on sound has developed with attention to this body of literature.

\section{Diaspora}


I employ the concept of diaspora to analyze Indian Tamil identity in the US. Diaspora is a complex concept because it can apply to many diverse groups and each diaspora has a specific historical context. Meanwhile, many scholars have competing views regarding the concept (Braziel, 2008). Some scholars view diaspora as a category, a term used to describe globally-dispersed communities that have an extended connection to a home (real or imagined) and maintain that connection over several generations (Brubaker, 2005; Braziel, 2008; Kalra, Kaur, \& Hutnyk, 2005). Other scholars, however, view diaspora as a community embedded in a complex process - one that may involve displacement/dislocation or legacies of colonialism or oppression that challenge the idea of belonging (Ifekwunigwe, 2003). My analysis emphasizes the latter definitions because they are often used by those who examine postcolonial diasporas.

Yet, even these definitions cannot encapsulate the multidimensional nature of diaspora. Equally complex is that diaspora overlaps with similar concepts like transnationalism, ethnicity, and at times, race, all of which I delve into further in Chapter 2 (Rozen, 2008; Braziel, 2008). Regardless of precise conceptual definitions, diaspora as a concept and category is used by both the many scholars who study Indian communities in the US (Bose, 2018; Lal, 2006; Mishra, 1996b; Safran et al., 2008; Sahoo, 2006; R. Sharma \& Annamalai, 2003; Skop, 2012) and the Indian government to refer to dispersed Indians abroad (HLCID, 2001).

\section{Goal of Dissertation}

My research broadly focuses on identity politics within diaspora communities. I provide insights into discrimination and othering in the US by critiquing generalizations and homogenized understandings of the South Asian/Indian diaspora more broadly and 
provide a unique analysis of identity through a pointed examination of the role of scale, hybridity and sound through an in-depth case study of Indian Tamil Americans. I argue that sound, particularly music, accent, language, and environmental sounds, is understudied in relation to identity but has a significant role in experiences of identity, including those connected to othering and discrimination. Understanding how identity is shaped, represented, and experienced drives my research goal.

My second goal is to challenge homogenization about Indian identities, reveal the complexity of experiences, and highlight how discrimination affects many Indian Tamils in the US. Focusing on the Indian Tamil diaspora exposes the diversity within Indian diasporic experiences in the US, demonstrating how these experiences both align with and diverge from academic, governmental, and popular generalizations of Indians. I examine how Indian Tamils fit into conversations of race in a post-9/11 and post-Trump election world.

This research enunciates 14 distinct findings, grouped into five specific areas. The first set of findings relate to the emergence of hierarchical scalar identities. The second set of findings relate to hybridity and the emergence of postcolonial identities. The third set of findings link hybridity and scale, demonstrating a complex relationship between these concepts. In some moments, often connected to discrimination, people think about identities as hierarchical. In others, they show that these identities are hybrid, in-between. The fourth set of findings focus on sound and identity politics. These findings suggest that sound is important to how identity is lived and represented. It also suggests that while discrimination is related to how someone looks visually (hair, skin, color), people also monitor their own identities and the identities of others through sound. Finally, discrimination is multi-layered as Indian Tamils experience and reproduce discrimination 
through sound at multiple levels within and outside of Indian communities as well as toward other minorities. These are also tied to geographic location, with many Indian Tamils experiencing more discrimination in rural, less diverse areas in the US.

I conducted participant observation, 55 in-depth interviews, and three expert interviews with participants who identify as Indian Tamil. These participants are from different generations and have connections to a variety of places in Tamil Nadu in New Jersey, Northeast Ohio, and Morgantown, WV. Two of my expert interviews were conducted in Chennai, Tamil Nadu and Tirunelveli, Tamil Nadu along with participant observation of music festivals in Chennai, Tamil Nadu. Through in-depth interviews and participant observation, I analyze identity in four ways. First, I examine how participants conceptualize identities through scale and hybridity, discussed in Chapter 4. Second, I look at sound's integral role in identity politics, discussed in Chapter 5. Third, I analyze discrimination through Indian Americans' experiences in US society in Chapter 6. Fourth, I analyze discrimination through internal identity politics within Indian communities in Chapter 7.

The first question I ask is how do scale, hybridity, and sound inform identities of Indian Tamil Americans? I examine how hybridity and scale are important to understanding how people navigate and conceptualize multiple identities within the Indian Tamil diaspora in the US. I also discuss that sound - music, accent, language, and environmental sounds - are significant to participant's diasporic identities.

Secondly, I ask, what insights do scale, hybridity, and sound lend to contextualizing discrimination for Indian Tamils in the US? By investigating the role of scale, hybridity, and sound to discrimination, I hope to provide a more robust understanding of discrimination in both the context of the broader US, but also within 
Indian communities. I show that participants find sound significant to experiences of discrimination and that the ways in which participants conceptualize identities will change with regard to their understandings of discrimination.

\section{Structure of Dissertation}

I structured this dissertation in 8 chapters. Chapter 2 covers the broader literature and scholarship for important theoretical concepts employed in my analysis. Chapter 3 is an overview of my case study, including a more detailed account of the history of the Indian diaspora. I also highlight my data collection methods, analysis, and positionality. I broke my analysis into Chapters 4 - 7. Chapter 4 examines the role of scale and hybridity in Indian Tamil American identities, while Chapter 5 addresses sound's role in these identities. Chapter 6 examines discrimination in the broader context of the US and Chapter 7 focuses on the role of discrimination in Indian and Tamil communities and subsequently, how these communities reinforce or mimic discrimination toward other minority communities. Finally, I conclude with Chapter 8, connecting my analysis from Chapters 4-7 and discussing the implications of scale, hybridity, and sound within identity politics. 


\section{Chapter 2 - Literature Overview}

In this chapter, I situate my research within broader theoretical frameworks. First, I define concepts such as hybridity, mimicry, othering, and in-betweenness. I then connect this literature to critical race and critical migration theory, and specifically to the concepts of discrimination and whiteness to homogenization. I follow with non-representational theory as it relates to sound. Finally, I discuss the concept of diaspora, connecting it to the postcolonial literature on hybridity and geographic literature on scale. Because the concept of identity politics is so robust, I outline multiple bodies of literature important to my later analysis. While the concepts of scale, sound, and hybridity overlap, I have placed them in these specific frameworks for readability of how each concept connects to specific bodies of literature. In this chapter, I provide an overview of significant literature, and in Chapters 4-7, I provide more specific connections of scale, hybridity, and sound to these bodies of literature

Postcolonial Scholars, Critical Race and Migration Literature, and Identity

Postcolonial scholarship is broad, but many aspects are relevant to my research, particularly as they relate to discrimination and Critical Race Theory. Postcolonialism broadly examines postcolonial effects of formerly colonized nations. I draw most significantly from Bhabha (1994) and his work on hybridity, mimicry, and in-betweenness. I also build on mimicry through Fanon $(1967 ; 1963)$, and Orientalism and othering through Said (1978; 1993; 1997). These scholars were also influential to Critical Race Theory, 
which I discuss later in this chapter. I do not give an overview of every important postcolonial scholar, but instead focus on those most relevant to my research. I also connect these scholars to geography and how geographical scholars have employed their work.

Postcolonialism has been influential across the social sciences and humanities, including analyses of diaspora and identity. Beginning in cultural and literary studies, it examines the complex, diverse effects and impacts of colonialism on colonialized peoples.

Fanon (1963) was one of the first major postcolonial writers. He addressed the impacts of violence of imperialism and nationalism to subdue the "colonial subject". He described how the colonizer used violence to keep the colonized oppressed - through physical violence, but more importantly, psychological violence. The oppressor, he argued, belittles and subjugates the "native" into feeling inferior or of lesser value than the colonizer. The colonized learned to mimic the colonizer, imitating psychological violence that the colonizers inflicted upon them. Fanon (1967) contributed to the founding of Critical Race Theory, showing how colonizers used race in tandem with violence and whiteness to keep certain groups and people subordinate (Rabaka, 2010). Race was another way colonizers justified both physical and psychological violence, keeping the colonized separate from and subordinate to whiteness (Fanon, 1967; Rabaka, 2010).

Fanon (1967) deconstructed whiteness and blackness, showing how these social constructions led to very real, embodied realities. He developed the idea of mimicry suggesting that mimicry is not just replicating violence, but also mimics the values of the colonizer through reproducing characteristics of whiteness in what he referred to as "black 
faces with white masks" (Fanon, 1967). In other words, the colonized mimic colonizer's behavior and embody characteristics of whiteness in order to gain legitimization or agency.

Fanon's overall work has invoked a variety of critiques including his focus on violence, patriarchal narratives, and a masculine-centered approach (Loomba, 1998). More relevant to my research is the critique that he represented the colonized as homogeneous, not recognizing the diverse experience of colonialism (Loomba, 1998) and that he relied on binaries, both in the binaries between the colonizer and the colonized and in terms of understanding race, particularly whiteness and blackness (Saldanha, 2007). In other words, he reified colonial binaries, even if he critiques them. Despite these criticisms, Fanon's $(1963 ; 1967)$ work has greatly influenced many of the scholars who theorize postcolonialism and critical race theory particularly Said, Bhabha, Saldanha, and others.

\section{Said and the "other"}

Said (1978) expanded Fanon's (1963) critique of the binary lines of colonized and colonizer through representational and discursive analyses. He developed the critique of othering and geographical imaginings, which were complex processes created by the West to dehumanize the "Orient" or the East. He suggested that the "Orient" was created through academic texts, literature, art, media, plays, and the news. As Said (1978) argued, the West's construction of the "other", the "East" and more recently the "Middle East" is intricately embedded into Western understandings of the world.

Said showed how the West has historically constructed the "East" or the "Orient" as exotic and backward through geographical imaginings. Said (1993) also showed how culture works simultaneously alongside empire to reify and justify these narratives. 
Imperialism is reproduced in "culture" with dominant narratives subsuming counternarratives (Said, 1993). Western sources like media, books, films, and art minimize counter, often non-Western narratives. Said (1997) also examined media representations and academic work to develop the concept of American Orientalism.

He builds on American Orientalism in 1997, focusing more specifically how Islam is represented through popular media. He argues that along with texts, art, media, plays and news, that current popular media contribute to "othering" Arab and other non-Western people (Said, 1997). He suggests that non-white faces and people, particularly those that are Muslim and brown, become synonymous with terrorism and this discourse affects the political climate of the United States and the world (Said, 1997).

Geographers and historians have expanded Said's work. Lewis and Wigen (1997) took Said's (1978) Orientalism and renewed it in a more geographical context. Instead of focusing on representation broadly through various arts and sciences, the authors hone in on geographical representations, map-making, and the specific act of shaping of boundaries. Lewis and Wigen (1997) argue that while geologically, tectonic plates could provide a source of current categorization of the map, the divides reflected in understandings of regions in the world embody Western narratives as the center of history and modernism, essentially "othering” the non-Western parts of the world. Gregory (2004) has further developed this work, suggesting that the "colonial present" continues through emphasis on Western narratives as the center of development and modernism.

Scholars have also critiqued Said because his argument rests on binaries of "East" and "West" or "West" and "other" (Bhabha, 1994; Loomba, 1998). Much like Fanon (1963), Loomba (1998) critiques him for creating the West as a static oppressor. While indeed 
these binaries were used by colonizers to legitimize conquering people, as Loomba (1998, p. 91) has suggested, "by exposing them, we risk reproducing them." In other words, bringing attention to the dangers of and effects of colonial binaries in some ways, reifies them. Thus, it is important to not just recognize colonial binaries of identities but bring attention to the ways in which identities are nuanced and to the ways that people experience identities. Some geographers have also noted that focus only on colonial texts and discourse overlooks the role of individuals (Haldrup et al., 2006). For example, Haldrup et. al (2006) bridge theoretical concepts like Orientalism (which often created binaries) with how people and individuals experience Orientalism in their everyday lives.

Bhabha critiques Said for "neat representations" of the colonizer and the colonized and that he misses the complexity of the colonial experience. Scholars like Bhabha (1994) reject neat representations in general, and colonial binaries more specifically because they miss the postcolonial condition of complex identities. Binaries do not reflect the rich, complex nature of identities, leaving out their "in-between" and "hybrid" nature, which Bhabha (1994) argues, are seminal to the postcolonial experience.

\section{Hybridity, in-between, and mimicry}

Homi Bhabha (1994) is formative to subaltern and postcolonial studies. Bhabha (1994) examines the shifting boundaries of culture and identity. Bhabha argues that colonialism reproduced binaries of colonized and colonizer, but simultaneously, created conditions whereby people's postcolonial identities became in-between and hybrid. I draw significantly on hybridity, in-betweenness, and mimicry in order to examine the complex 
nature of especially post-colonial and diasporic identities (Gilroy, 1993; Hall, 1996; Sharp, 2011; Sparke, 1998; Spivak, 1988; Young, 1995).

Bhabha (1994) challenges traditional binaries associated with colonialism - the divisions of the colonized and the colonizer into neat categories. He suggests that in a postcolonial world, identities and cultures are much more complex. Hybridity, or the idea that identities and cultures are in-between binaries, is important for Bhabha.

Hybridity dismantles the idea of cultural homogeneity that was reinforced by colonial powers. Colonial powers reinforced such ideas of identities in order to maintain power over the colonized. Cultures and identities are not fixed, nor static. Bhabha (1994) equates this to a post-colonial condition where traditional binaries and lines of cultural purity or authenticity reinforced by colonizers are imagined. The colonizers used binaries as tools to keep them in power, yet identities born out of postcolonial conditions are in some ways, in-between binaries of colonized and colonizer (or West/East). Postcolonial identities are hybrid spaces of in-between that often go unrecognized and unacknowledged. Hybridity, for Bhabha (1994) is a space of "in-between" - neither here nor there, and most certainly not pure.

Geographers like Sparke (1998) draw on Bhabha while also challenging aspects of his work that suggest that hybridity and in-betweenness provide agency for identities., Sparke (1998) argues that in-between spaces are still constrained by colonial binaries. Sparke's (1998) case study focuses on a trial brought against British Colombia through two First nations - the Gitxsan and Wet'suwet'en, regarding their sovereignty. They attempted to use oral traditions in the court setting, which was not recognized by the federal and provincial government as a legitimate source. According to Sparke (1998), the performance 
of culture in the courts reinforced binaries. While the Native, non-colonial names were plotted on maps, the act of using maps confined expression to a colonial system. In other words, Sparke (1998) shows that the Gitxsan and Wet'suwet'en are bound to the legacy of colonial binaries even as they seem to actively and directly challenge colonial binaries. Sidaway (2000) has also shown how colonial practices are still embedded and visible in international relations that reify the colonial system. He suggests that even the most critical geographers are trapped by the colonial past (Sidaway, 2000).

Other scholars have built on the work of Bhabha (1994) to expand the definition of hybridity and situate in-betweenness geographically through scale, place, space, boundaries, and cartography. Sharp (2011) draws on "in-betweenness" in geopolitical case study of Tanzania. Sharp (2011) looks at a specific place, Tanzania, which she suggests is on the outskirts of power, both in discourse and in the literal geographic sense. She argues that in the United States, "the War on Terror" is framed as a binary - between the United States and the "Middle East". Tanzania serves as a place of "in-betweenness" from which to view the war on terror. She acknowledges the "danger" of essentializing and creating the very binaries that she attempts to deconstruct, but nevertheless, demonstrates the concept of "in-betweenness" in a grounded, material way.

Harris (2014) has also expanded the discussions of hybridity by drawing on emotions and everyday lives to examine narratives of environment within Turkey. She shows how binaries of East and West are actually quite complex and in-between, especially regarding people's identities. She further describes that environmental sites in Global South still continue to "intersect with colonial and power-laden logics and pathways," not just in discourse, but in everyday emotions and identities (L. M. Harris, 2014, p. 811). In 
other words, spaces of in-between are influenced by binaries, but nevertheless, remain hybrid, "in-between" and complex, situated by factors like emotion.

Hybridity is often used as a way to describe identities are not fixed, static, bounded by solid borders, and do not originate from a single source (Gilroy, 1993; Kraidy, 2005). For Chacko and Menon $(2013,99)$, hybridity "destabilizes fixity." Kraidy (2005, p. vi) describes hybridity not as a single idea or a unitary concept, but as "an association of ideas, concepts, and themes that at once reinforce and contradict each other." Academics have used hybridity, as Bhabha (2015) and Chacko and Menon (2013) have noted, as a stand-in term for the blending of identities and as a way to avoid binaries. Yet, hybridity is not an alternative classification to rectify binaries. As Bhabha (1994, p. 112-113) indicates, "Hybridity has no such perspective of depth or truth to provide: it is not a third term that resolves the tension between two cultures..." Simply put, hybridity is not just a term that describes in-betweenness or blurred identities. Instead it implies blurred, in-between (binaries), but also must be understood as a product of colonial past that influences the present.

For Bhabha (1994, p. 114) hybridity is always tied to the colonial legacy, connecting to how identities are represented by the colonizer and how the colonizer navigates and maintains control:

These metaphors are very much to the point, because they suggest that colonial hybridity is not a problem of genealogy or identity between two different cultures which can then be resolved as an issue of cultural relativism. Hybridity is a problematic of colonial representation and individuation that reverses the effects of 
the colonialist disavowal, so that other 'denied' knowledges enter upon the dominant discourse and estrange the basis of its authority - its rules of recognition.

Hybridity is connected to a colonial past and thus, the popular and societal rhetoric reifies colonial binaries, much like Harris (2014) suggests, also surfaces within people's everyday emotions and identities. Colonial binaries are not just limited to broader discourse or the historical past of colonialism. In fact, these binaries can be embodied, lived, and reified because colonialism legacy is inescapable (Sparke, 1998; Harris, 2014).

Hybridity is also embodied in everyday lives through mimicry. The formerly colonized mimic the colonizer's behavior to gain power or benefits (Fanon, 1967; Bhabha, 1994). Yet, as Bhabha (1994) explains, the colonized never have the power of the colonizer, they only appear to have power. Mimicry reflects a "doubleness" where the formerly colonized appear to have power, but simultaneously occupy the space of being colonized. This, Bhabha (1994) refers to as embodying a "double" or hybrid identity.

As Bhabha (1994) and Fanon (1967) describe of mimicry, it is related to whiteness. "Almost the same but not white: the visibility of mimicry is always produced at the site of interdiction" (Bhabha, 1994, p. 89). In short, the formerly colonized mimic the condition of whiteness, even if they never maintain full privileges of whiteness. For example, Jazeel (2006) draws from Bhabha's (1994) concept of mimicry to show how the privileged wave of diaspora emerged from the colonial sphere - specifically, the Sri Lankan diaspora in the Sri Lankan Women's association in London. The official Sri Lankan diaspora association maintains and reinforces gender roles set by the colonial mentality before independence women and men are separate and designated with specific tasks. He focuses more 
specifically on how the women mimic a colonial role as the guardians and protectors of underprivileged. These women occupy a double, hybrid identity in that they view themselves as exoticized and Orientalized, but also take on the role of the colonizer through othering and exoticizing Sri Lanka.

Many geographers have commented on concepts like hybridity, mimicry, and "inbetween," but have also advocated for new directions in postcolonial geographies (Gilmartin \& Berg, 2007; Nash, 2002; Sharp, Briggs, Sharp, \& Briggs, 2006). Gilmartin and Berg (2007) call for more inclusive postcolonial geographies, discussing how postcolonial geography is represented by primarily white scholars while non-white scholars remain on the periphery. Nash (2002) argues for further grounding postcolonial geography within the lives of everyday people, suggesting that it has focused too much on text and discourse. Sharp and Briggs (2006), reflecting some of Nash's (2000) sentiments, discuss the gap between development studies and postcolonial theory. They suggest that postcolonial theory, in the literary sense, is too removed from reality and everyday people, and development studies, though used more often, simply reinforces colonial relationships of power. Recently, scholars have called for decolonizing work that focuses on the perspectives and agency of the (de)colonized (Harris, 2014).

Concepts like hybridity, mimicry, and "in-between", though not without criticism, are critical to examining the Indian diaspora in the United States. The colonial legacy as Sparke (1998) suggests is embedded in the very structure of the world today and still affects and shapes how scholars and the people scholars study understanding identity today regardless of location. Often, as Jazeel (2006) suggests, the Indian diaspora that is in a more privileged position than other minorities can embody and mimic colonial attitudes, 
also essentializing and exoticizing aspects of "South Asianness". Postcolonial applications are incredibly valuable to my research, but concepts like mimicry and "otherness" overlap with whiteness as described by Bhabha, Fanon, and other scholars. Thus, critical migration literature and Critical Race Theory is similarly important to situating research on identity and also connects to literature on postcolonialism.

\section{Critical Migration Literature and Critical Race Theory}

In recent political climates post-9/11, US discourse has focused on the legality of citizenship and the policing of immigrant bodies (Jones, 2012, 2016; Mountz, 2010). As Hyndman (2012) indicates, the geopolitical "war on terror" transitioned to become biopolitical, focusing on the direct policing of immigrant bodies (Hyndman, 2012). Feminist approaches to migration such as those of Hyndman (2012) have shifted focus from larger geopolitical narratives of migration to focus on body politics of migration and immigration.

Body politics with regard to migration and foreign bodies have become increasingly connected to race and whiteness post-9/11 and post-Trump election (Gökarıksel \& Smith, 2016; Steinberg et al., 2018; Winders, 2005). The immigrant body is increasingly profiled, monitored, and policed (Mountz, Coddington, Catania, \& Loyd, 2013; Silvey, 2014). "Illegal" bodies are often depicted as ethnic, brown, black, or "other," in dominant in US discourse (Ngai, 2004; Winders, 2007, 2008). For example, non-white and immigrant bodies are central to xenophobic and racist narrative supported in Donald Trump's presidential campaign and current administration (Gökarıksel \& Smith, 2016; Steinberg et al., 2018). The Trump administration discourses use words like "legal" and "illegal" as a way to emphasize differences between white and non-white bodies specifically through depictions 
of people from the "Middle East" or from Central and South America, as terrorists or criminals (Gökarıksel \& Smith, 2016; Steinberg et al., 2018). Distinctions between whiteness and non-whiteness in political rhetoric on immigration and immigrant bodies demonstrates a link to racial politics (Dave, 2013; Winders, 2008).

Critical Race Theory (CRT) has helped to develop critical migration literature by building on concepts of othering, hybridity, and mimicry. CRT emerged in the 1970s after the Civil Rights movement as scholarship that disrupted traditional approaches to race (Delgado \& Stefancic, 2017). It suggested that although race is socially constructed, it has real, embodied effects on people's everyday lives (Delgado and Stefancic, 2017). Fanon's (1963; 1967) work on the colonial subject and race heavily influenced CRT (Rabaka, 2010). Black Skin, White Masks, demonstrates that blackness and whiteness are sociallyconstructed, but yet were used by the colonizer to dehumanize and subjugate the colonized (Fanon, 1967; Rabaka, 2010).

CRT has developed into a robust body of literature maintaining that even socially "progressive" approaches, or those that advocate reforming society and promoting greater equality have been regressive and silenced minorities (Delgado and Stefancic, 2017). CRT scholars critique "progressive" politics for ignoring color and race and thus perpetuating racial inequality. In other words, race heavily impacts the lives and bodies of people of color daily (Joshi, McCutcheon, \& Sweet, 2015). CRT scholars critique the idea that the US is a "post-racial" and equal society. The idea of equality masks discrimination because it homogenizes, suggesting that everyone has the same experiences. "Equality" ignores that people of color or marginalized groups face discriminatory practices and institutional racism (Delgado \& Stefancic, 2017). CRT scholars suggest that ignoring color and race is not 
possible for people of color, because they experience the effects of race daily. Simply put, people of color do not have the privilege to ignore race. Pretending to not see color or moving toward "lack of race" defines white privilege and ignores the implications of race in society (Delgado and Stefancic, 2017).

Building from CRT, Whiteness Studies developed to examine white privilege, or the privileges of white as the default norm, including that whiteness has the power to ignore race altogether (Bonds \& Inwood, 2016). It suggests that when people do recognize race, they do so in relation to non-white bodies. The condition of whiteness provides privilege to ignore color, because after all, color is based on the condition of being a non-white norm, as Bonds and Inwood (2016, p. 717) describe:

This emphasis calls attention to the simultaneous invisibility and ubiquity of whiteness as a racial position, such that the notion of 'race' is applied almost exclusively to non-white people. It reveals how whiteness acts as the unseen, normative category against which differently racialized groups are ordered and valued.

Simply put, whiteness is normative. Non-white exists as a category simply because it is compared to a default category - that of white. Richard Dyer (1997) demonstrates in his seminal text White, how the concept of "whiteness" is also socially constructed. It has evolved through film, media representations, and histories of colonialism to become ubiquitous with purity, non-race, and the standard to which all other racial categories are measured (Dyer, 1997). Saldanha (2007) comments on the significance of White by Richard 
Dyer in relation to geography and the social sciences. Saldanha (2007, p. 7) particularly draws on the concept of whiteness as outlined by Dyer (1997):

What then counts, in human geography, cultural studies, anthropology, and sociology, is often the discourse on, media images of, people's opinions about race, instead of the realities of embodiment, face, and location...The work of Dyer and others in white studies has been valuable in exposing how whites have historically erased their own racial specificity. Although blacks and reds are colored, that is, deviations from white, whites are just human. Humanity itself is defined on white terms.

Saldanha, echoes many other CRT scholars, pointing out that whiteness is norm whiteness is human. Thus, non-white, immigrant, or 'illegal' bodies become inhuman (Gökarıksel \& Smith, 2016; Steinberg et al., 2018). Saldanha and other CRT scholars stress the importance of actively recognizing when and how color becomes subsumed by default whiteness. Privileging whiteness as the norm renders people of color invisible and ignores how non-white bodies are depicted as inhuman and actively discriminated against (Saldanha, 2007; Winders, 2008). Whiteness also creates dichotomies (i.e. white and nonwhite) that erase hybrid and fluid identities. Binaries, as Fanon $(1963 ; 1967)$ and Bhabha (1994) argue, are also remnants of colonial legacy. Non-white groups become homogenized or misrecognized precisely because they are defined in relation to whiteness (Hopkins et al, 2017). Specificity, diversity, and individual experiences are marginalized and depicted as simply an "other". 
Whiteness studies has also demonstrated how groups, often brown or "ethnic", have become associated with "whiteness" because of economic success (Inwood \& Bonds, 2016). Bhatia (2007) refers to this condition as "brown privilege." Bhabha (1994) connects the privilege of whiteness to mimicry. The colonized mimic the colonizer in behavior, trying to attain the privileges associated with whiteness. In part, this is connected to binaries. As Bhabha (1994, p. 61) describes "turn white or disappear", people of color, often coming from a legacy of colonial exploitation, are forced to choose between whiteness or non-whiteness, but not both. Though the colonized may attempt to become the colonizer, they are always the colonized (Bhabha, 1994). In other words, despite that some groups strive to fit into "whiteness" or economic privilege and power, they are still brown and thus are viewed as non-white bodies (Chand \& Tung, 2014; Dave, 2013).

Stereotypes and myths, such as that of the model minority, affect those in "brown" communities (Dave, 2013; Hopkins et. al, 2017). Narratives of success can silence minority voices. For example, conflating model minority success with the Indian diaspora silences voices of those who are undocumented, face growing anti-Muslim violence, or who are struggling to survive (Passel \& Cohn, 2016; Sridaran et al., 2017). Binaries of black and white are problematic for many communities who do not fit within either of these categories. As many scholars have noted, tensions between being black and white in the US often presented as dichotomies, are problematic for those who are categorized by the American public as "brown" (Bhatia, 2007; Kurien, 2005; Sridaran et al., 2017).

Whiteness involves much more than skin color (Dyer, 1997). Whiteness as a social condition has been linked to accent, language, music, and even tonal sounds of speech (Haldrup et. al, 2006; Simonsen, 20120; Kanngieser, 2012; Dave, 2013). Kanngieser (2012) 
describes how accent and perceived speech tones can indicate whiteness or blackness. Dave (2013) describes how accents show non-whiteness through foreign bodies. She shows how Indian accents are used as racial markers that denote difference between white and foreign, "other" bodies. Haldrup et. al (2006) and Simonsen (2010) show how "othering" non-white bodies is a multi-sensory experience that relies on sound, smell, and even taste. I build on this literature in the subsequent section and further in Chapters 6 and 7.

\section{Non-representational theory and Sound}

Postcolonialism, critical race, and critical migration literature is important to how identity is represented, constructed, lived, and experienced because it demonstrates how pervasive whiteness and legacies of colonialism inform identities in current political contexts. Non-representational theories, have much to offer in regard to how people experience identity in relation to concepts like hybridity, "othering" (Haldrup et. al, 2006; Saldanha, 2007). Much conceptualization of identity, people, or places comes from representational sources like film, media, maps, images, and discourse (Rose, 2016). Many, but not all, of these representational sources overlap with literature on colonialism, Orientalism, and "othering" (Rose, 2016). Non-representational theories (NRTs) brings the focus to everyday experiences of people including their senses like sound, smell, taste, or their emotions and memories (Thrift, 2008). NRTs brings attention to individuals, emotions, subjectivity, and how people experience identity through the sensory - the tactile, visual, and aural aspects of the moment (Thien, 2005; Tolia-Kelly, 2006). Nonrepresentational theories contribute to and underscore the everyday, lived experience, and 
practice, not often emphasized in the past wave of "critical geographies" like postcolonial geography.

Postcolonial geography, though many geographers have recently applied it to everyday and lived experience (Haldrup et. al, 2006; Simonsen, 2010), once focused more on text and representation than everyday experience (Nash, 2002). Saldanha (2007) for example, grounds his research on critical race theory and postcolonial concepts of "othering" through non-representational theory, focusing on affect and experiences of bodies. He shows how "brown" bodies experience being ostracized and differentiated in white musical spaces in Goa, India. NRTs provide a theoretical contribution as well as a style of thinking that values practice, performance, and the everyday experience. As Pile (2010, p. 5) notes, the field of NRT continues to grow and "geographers have not only taken up a variety of positions, they have also shifted position over time". The development of NRTs intersects with phenomenology, post-structuralism, actor-network theory, feminism, and sociology (Anderson, 2009b). Within geography, scholars such as Thrift (2008) and Anderson (Anderson, 2009a) look at broader applications of bodies using affect or emotions from multiple bodies (i.e. emotions in unison), while others such as Thein (2005) and Davidson and Milligan (2004) employ a feminist lens to highlight the importance of emotion, subjectivity, and the individual.

The theoretical contribution of NRTs emphasize moments and experiences in a discipline that previously emphasized discourse, text, and visuals (Doel, 2010). They also provide methodological contributions that accentuate people's everyday experience over how they are represented (Doel, 2010). Representations can render life experience as "inert", but NRTs look at living for the sake of the moment and living for the sake of living 
(Dewsbury, 2010; Doel, 2010; Harrison, 2010; Lorimer, 2005; Thrift, 2008). The sensory highlights individual experience and how people process, understand, and live in moments (Thrift, 2008).

Phenomenology, arguably paved the way for non-representational theory (Pickles, 2009). Phenomenology first emerged as a philosophical framework the 1700s, but found its way into Geography in the 1970s (Pickles, 2009). It emerged in Geography as a critique of Marxist and structuralist approaches that thought of people and places as abstract (Pickles, 2009). Phenomenology emphasized the experience, the individual, and human subjectivity (Farina, 2014). Phenomenology scholars like De Certeau (1984) particularly stressed the importance of the everyday within social science. He described discourse as only part of the story and that mundane activities in daily life were a means to perform or exercise as an act of resistance against particular discourses. Discourses, as de Certeau (1984) saw them, only told one side of the story that ignored the everyday individual.

Scholars in geography like Saldanha $(2007,2010)$ and Anderson $(2009 a)$ rely on NRTs for their research. Saldanha (2010) research in Goa, India (2007) shows how race is constructed through a variety of factors such as skin color, emotion, and spatial inequalities played out by bodies. He describes race as an event that relies on bodily interaction and practice, demonstrating how material effects of emotion and spatial inequalities are Indian bodies being othered in Goa's white rave scene. In other words, Indian bodies are represented as other, but also these bodies experience everyday, material impacts of "othering" discourse.

Anderson (2009a) uses NRTs through applying 'affect', a culmination of emotions and experiences, in material observations, to his research. He (2009a, p. 80) suggests that 
"affective atmospheres emanate from the assembling of the human bodies, discursive bodies, non-human bodies, and all the other bodies that make up everyday situations", but have real, material effects in the landscape. He provides examples such as protective atmosphere like gated communities or inhibitive building designs or even concert halls and sports stadiums that encourage a specific affective response in unison, i.e. crowd excitement or cheering. Inhibitive elements of the landscape can also be created in a space, such as monetary power to gain access to a cultural event.

Thrift (2008), Anderson (2009a), and Saldanha (2007)(2010) have broadly theorized emotions as affect or how emotions such as anger, fear, happiness, etc. work together and play on other people's emotions and bodies. These create "atmospheres" that are "reducible to bodies affecting other bodies, yet exceeding the bodies they emerge from" (Anderson, 2009a, p. 79). In other words, atmospheres are made of multiple bodies, emotions, and they affect bodies, but they are also move above and beyond the confines of bodies. Affective atmospheres diffuse spatially and work as an assemblage through which to engage with space in a material, grounded way (Anderson, 2009a; Anderson \& McFarlane, 2011).

Some scholars have openly criticized NRT approaches for representing when it claims to not represent. "Apologies for being blunt, but this is a straightforward hypocrisy. It continually does what it says cannot be done: it cannot help but represent and represent affect - and in language" (Pile, 2010, p. 17). Responding to this criticism, some have pointed out that non-representational theory's goal is to try to not represent (Thrift, 2008), others have used it to move away from representation and focus on experience that had been previously overlooked (Harrison, 2010) or place limits on representation (Lorimer, 2008), 
and some highlight the importance of understanding that representation is merely a representation of a moment (Doel, 2010).

Feminist lenses on NRTs fill in the personal, subjective, case-by-case, individual, and emotional elements. Feminist scholars have contributed significantly to development of the emotional aspect of NRTs. Many have pointed out that focus on affect reflects masculinized "detachment, objectivity, and rationality", while "engagement, subjectivity, passion, and desire" have been feminized and thus, undervalued in geography (Anderson \& Smith, 2001, p. 7; Nash, 2000; Smith, 2011; Thien, 2005; Tolia-Kelly, 2006). Some scholars have openly critiqued the affective approach to NRT with the notion of it being a more "objective" way of viewing emotions (Anderson \& Smith, 2001; Lorimer, 2008; Thien, 2005; Tolia-Kelly, 2006). Some argue that these theories can be dangerous if employed as "objective" and "distancing", i.e. broad, sweeping generalizations of affect or emotion within cities such that Thrift (2008) employs that detach emotion from the individual person (Tolia-Kelly, 2006).

Feminist scholars have also suggested that broader affect can also be "Westnocentric" and "universalist" (Thien, 2005; Tolia-Kelly, 2006). Thrift (2008) addresses this point, noting that many examples of affect come from "European cities" that may in fact be "Anglo-centric", but acknowledges that emotions differ across "cultures,"; however, he his case studies are European cities. Sharp (2009) describes the danger of Thrift's broader affective theories as leaving out the personal, emotional, and subjective nature of affect, which is a key component of feeling. Additionally, current affective framework is often universalizing and does not always challenge power and knowledge hierarchies (Sharp, 2009). 
Emotions, subjectivity, and arguably creativity are very much a part how nonrepresentational theories contribute to understanding the world (Wylie, 2010). Overall, many feminist critiques have influenced and developed other aspects of NRTs. Lorimer (2008) describes how feminist lenses have been important to NRTs, especially in keeping affective geographies grounded and not too apolitical. Both can be utilized to understand the everyday, practice, and lived experience, without continuously separating them in academic debates. Anderson (2009a) suggests that affective atmospheres do understand the individual, subjective nature as affective geography seeks to understand a body as well as multiple bodies.

These theories address the sensory with regard to sound, smell, touch, movement as well as emotions (Thrift, 2008; Wylie, 2005; Zeitlyn, 2013). For example, identity can be understood beyond a representation, but also as an embodied experiences, rich with emotions and senses (Dewsbury, 2010; Thrift, 2008).

\section{Sound and identity}

"Most of us live complex daily lives that are often more than not experienced through varied competing auditory and visual representations..." (Aitken \& Kwan, 2010: 287).

Of particular importance to my research is the connection between identity and sound. Non-representational theorists have discussed the importance of senses such sound to identity and broader affective atmospheres (Anderson et al., 2005; Jazeel, 2005; Thrift, 2008). Geography has been a traditionally visual discipline and much of geography is 
grounded in visual analysis (Anderson, 1987; Groth, 1997; Rose, 2012; Sharp, 2011; Sparke, 1998) as NRTs show. However, sound provides key insights for many research projects (Pijanowski et al., 2011). For example, in a seminal study on sound referenced by subsequent soundscape scholars, Pijanowski et al. (2011) demonstrated that sound is a key indicator of environment health. Examining the Costa Rican rainforest, Pijanowski et al. (2011) found that visually, past and present images of the forest were similar. However, when they collected environmental sound data, they found that many species were missing from the environment (Pijanowski et al., 2011). They suggest that without the sound analysis, they would have overlooked the negative impact of logging on biodiversity within the Costa Rican environment (Pijanowski et. al, 2011).

Pijanowski et al.'s (2011) study focuses on environmental sounds related to broader environmental studies; yet sound has also been hailed in research in human geography. Scholars like Revill (2016), Kapchan (2016), Kanngieser (2012), Smith (1994; 2000), Jazeel (2005), and Anderson (2005) have shown how sound is crucial to political, social, cultural, and human environments, but do not focus on identity. Beyond geography, much literature has been written on sound and soundscapes (Jazeel, 2005; Miller, 2008; Pijanowski et al., 2011; Raimbault \& Dubois, 2005; Revill, 2016; Smith, 1994; Smith, 2000; Waterman, 2006). Southworth (1969) is credited for identifying the importance of sound in the urban landscape. What he refers to as a "sonic sign," or the awareness of a sound, creates visual images in the viewers' mind that reinforce an image that they see through sound that they hear (Southworth, 1969). Visuals are part of fluid and complex cultural landscapes that involve multiple senses (Longstreth, 2008; Riesenweber, 2008; Wylie, 2005). The viewer 
sees the image, but experiences memories and emotions to create or re-create an experience through sound, smell, taste, and touch (Wylie, 2005).

Many scholars have recently written on sound and geography, thus facilitating an important turn in geographic literature (Anderson et al., 2005; Jazeel, 2005; Revill, 2016; Saldanha, 2005; Smith, 1994; Smith, 2000; Waterman, 2006). As many NRT scholars show, sound also affects how many people conceive of space and place. Scholars like Dewsbury (2010) or Wylie (2005) highlight how sound is not only important to understanding place, but also experience. Wylie (2005) clearly and distinctly highlights how sounds made him feel uneasy and panicked during his walk through the woods to the coast on the South West Coast Path in England. In one example, Wylie (2005) is able to link sounds to memories and past experiences. "Suddenly the morning silence of the forest was broken by a cry. A loud, ululating cry, one which perfectly mimicked, in every detail of pitch, variation and length, the cry of Tarzan, lord of the jungle, familiar to me from old Saturday morning black-andwhite serials" (Wylie, 2005, pp. 238-239). In other words, sounds influenced how Wylie (2005) perceived and understood his environment. Scholars refer to sounds that affect an environment or trigger emotions and memories as environmental sounds (Wylie, 2005). Many scholars have also examined sound through music its emotional effects on listeners and/or bodies (Anderson, Morton, \& Revill, 2005; Gilroy, 1993; Jazeel, 2005; Saldanha, 2007; Sharma, 2006; Smith, 1994, 2000). Saldanha (2007) describes the music of the rave scene and how it creates racially-constructed space, demonstrating that sound and race are connected. As Saldanha (2007) shows, white bodies and brown bodies are visibly separated but simultaneously entranced by rave music - which is often associated with whiteness and privilege. Thus, white rave music creates a space where whiteness is 
privileged over brownness. For the white patrons of these bar scenes, brownness disrupts the white, aural atmosphere and thus, brown bodies are ostracized and separated from white bodies.

Music can even "disrupt ideas of purity and origins" to demonstrate hybridity (S. Sharma, 2006, p. 318). Sharma (2006) shows this through his case study of British and Asian fusion music. Bhangra music embodies hybridity in the Punjabi British diaspora that fuses traditional folk sounds with some Western elements. Sharma (Sharma, 2006, p. 324) suggests that the music goes beyond the "mainstream" to become an important part of "a lived diasporic identity". Music is a way, according to Sharma (2006, p. 324), to affirm an "Asian identity" and "agency" while also "responding to the harsh realities of a multi-racist Britain". BrAsian music "defies national boundaries and cultural authenticities" (Sharma, 2006, p. 325) to be a source of resistance to homogenizing Eurocentricness.

Attali (1985) describes sounds such as music or language as "organization of noise". He suggests that unorganized sound is often deemed as "noise" (Schafer, 1994). Noise resembles chaos, something that is hard for the human brain to understand or manage, which is why humans gravitate toward organized noise (Attali, 1985; Grosz, 2008). But, music and language are more than just organized noise - they are "humanized sound(s)" that reflect human emotions and thoughts (Waterman, 2006, p. 1). Music and language create pleasure, connecting people to the most basic feelings (Grosz, 2008). Sounds open up human awareness to emotions, feelings, and moments (Smith, 2000).

Scholars have also studied sound with regard to language and identity (Anzaldúa, 1987; L. D. R. Jones, 2001; Rhys Jones \& Merriman, 2009; Paasi, 2003; Segrott, 2001); particularly, how languages reinforce, assert, or cultivate specific identities (Jones \& 
Merriman, 2009; Paasi, 2003; Segrott, 2001). Language is often viewed as a signifier with intended meaning and significance (Rose, 2016). Dewsbury (2010), however, building on NRTs, moves from language as a signifier to language as a raw experience. He advocates for understanding language and words as an "experience and event", rather than focusing on their inherent meaning alone. To demonstrate, he describes the difference between doing art and a work of art - one is the act, the other is a representation of a moment. He suggests that language can be looked at as a representation, but it also must be examined as an event within a moment - this is we experience language in tones, timbre, tonality, inflection, pitch, and texture.

Kanngiser (2012) builds on sound through focus on accent, explaining that geography has not examined how people "listen". For example, she shows how Obama's perceived speech tones as "black" or "white" were heavily criticized during his campaign. But she describes that accent and perception of speech also involve tone. She explains how higher-pitched, softer voices are thought of as feminine, while lower pitched, louder voices are thought of as masculine. The loudness of a voice can also determine power, while silence can be a sign of protest. She posits that " $(t)$ he inflections and modulations of the voice contain forces that we must become more conscious of" (Kanngieser, 2012, p. 348). Accent, for Kanngieser (2012) is equally important to sound as music, language, and environmental sounds.

Dave (2013) suggests that accents are generally compared to what is considered standard, normal white speech and key to the process of "othering". Non-white accents are deemed by white dominant groups as "other" and even foreign. She provides the example of the typical "Apu" accent from The Simpsons that has been used to not only differentiate 
Indians, but also ascribe a singular accent to a diverse body of people who have many different "Indian" accents. Additionally, she analyzes the American accent, though it differs between regions and is often defined by broadcasters and Hollywood in a form of Standard American English, as a way to reinforce a singular definition of American identity - one that limits participation from those who speak in "foreign" or nonstandard accents (Dave, 2013).

Haldrup, Simonsen, and Koefoed (2006) and Simonsen (2010) bridge Orientalism to everyday experience through music, accent, language, and environmental sounds. They examine material effects of "othering" in what they term "practical orientalism", that is the lived, embodied, and experienced process of othering in "everyday life". They look at how people in Denmark use multiple senses to "other" those of Middle Eastern origin. More specifically, they look at how food, smells, sounds, and tastes can create an image of the "other" in a very real, lived way. For example, they show how the Danish described the sounds of Arabic or the sounds of the Islamic call to prayer as threatening, not pleasant, harsh, and even abusive. In essence, sounds were given and ascribed specific qualities in lived experiences.

Sound is not just important to lived experience and NRTs, it is also important to representation through film and other media (Dave, 2013). Samuels et al. (2010) demonstrate how film incorporates both an audio and visual narrative. Generally, sounds incorporated into films requires listeners focus on particular sounds and textures to enhance a story or broader narrative (Samuels et al., 2010). For example, in various cinemas throughout the world or performed reenactments, depiction of past wars incorporate both visual images of battle and sounds of explosions, firing guns, or clashing 
metal swords. Celebrations also incorporate sounds - The Fourth of July reenacts the War of Independence for the United States through booming fireworks, and the Christmas season all over the world has evolved in many ways to signify sounds such as bells or chimes.

Likewise, fictionalized books describe such sounds - from sounds of muffled motors or whizzing cars, to descriptions of battle with clashing swords, or even in the more mundane soft whisper or tone of a person's voice as they speak in a narrative. Even in today's journalistic practices, it is increasingly common to convey both the visual information of a photograph and the aural and environmental sounds to give the most comprehensive depiction of an event (Jenkins, 2007). Sounds are key to individuals' experiences, and are engrained in our existence, politics, and experiences (Revill, 2016; Smith, 2000), yet sounds connection to identity, othering and discrimination are just emerging.

Waterman (2006, p. 1) indicates, “...hearing and listening are both vital in the mediation of ideas and the transmission of culture. Senses of hearing (and smell) are capable of evoking memories and images more powerfully than things we see..." Music, language, accent, environmental sounds, and even representations of these elements in films or national dialogues are key to conceptualizing how communities use sound to express, recreate, shift between, or solidify identities. As many scholars have compellingly noted, sounds are extremely important to our daily experiences, politics, performances, and identities. Particularly in communities and diasporas, sounds play a strategic role in navigating, articulating, and defining identities. 


\section{Diaspora - Origins and traditional definitions}

Because it is contested, diasporic literature has followed many trajectories (Brubaker, 2005; Gilroy, 1993; Vertovec, 1999). Diaspora emerged from the study of Jewish exile, but now describes many types of communities and identities (Braziel, 2008; Dufoix, 2008). Brubaker (2005) identifies diaspora as a "way of formulating the identities and loyalties of a population." Brubaker (2005, p. 2) suggests that "its meaning has been stretched to accommodate the various intellectual, cultural and political agendas in the service of which it has been enlisted." Brubaker (2005) further states that diaspora has evolved into multiple definitions.

First-wave diaspora studies use diaspora as a category by which to describe a group of people (Brubaker, 2005). This definition acknowledges but does not always examine colonial context or power relations. Rather, it focuses on the growing connection of individuals based on post-1990's globalization, suggesting that diaspora dispersion is a direct outcomes of global capitalism and the expanse of the global labor market (Braziel, 2008). The Internet, information communication technologies (ICTs), and decreasing costs of travel have created diasporas (Kalra et al., 2005). Because of these things, people are more likely to migrate and disperse (Carles et al., 1999). ${ }^{7}$ While these reasons are indeed important to current diaspora dispersion, they still lack a postcolonial context (Dufoix, 2008). As Cohen (1997) suggests, globalization has not created diaspora, but rather augmented its presence as a "form of social organization".

\footnotetext{
7 It is worth noting that this is not the case for all people and is dependent upon context and agency
} (Mishra, 1996b). 
A second approach to diaspora focuses more on the politics of diasporic identity, often linked to colonialism, an approach that I take in my own research. This approach falls into the second-wave of diaspora studies. It suggests a non-static process or condition driven by a journey of collective identity that examines effects of power relations and colonialism on current diasporas (Kalra et al., 2005). Here, Bhabha's (1994) hybridity is key. These scholars critique the first definition of diaspora as being over-categorical, lacking colonial context, and masking the experiences of those in the diaspora, reducing them to only a category (Mavroudi, 2007). The colonial concept of "cultural purity" creates problematic classifications and definitions of diaspora (Kalra, Haur, \& Hutnyk, 2005). Culture is not pure is always influenced and hybrid (Bhabha, 1994). Colonial powers often used the idea of cultural purity to distinguish and marginalize people to gain power (Bhabha, 1994). The focus on the colonial implications are crucial to the second approach to diaspora. This approach also focuses on individuals and emphasizes storytelling about daily lives and experiences (Hartmann, 2008), crucial to my research on the Indian Tamil diaspora.

\section{Second-wave diaspora definitions}

The first wave of diaspora studies views diaspora as a category, but the secondwave views it as a process, intertwined with the aftereffects of colonialism (Bhabha, 1994; Dufoix, 2008; Gilroy, 1993; Hall, 2003; Hartman, 2008; Ifekwunigwe, 2003). First-wave diaspora scholars have critiqued second-wave diaspora studies. They are critical, arguing that it is too broad and limits the usefulness of diaspora (Braziel, 2003; Brubaker, 2005). Brubaker (2005, p. 2-4) suggest that diasporas were once "firmly rooted in the concept of 
homeland" but now include many types of communities, making the meaning of diaspora almost "useless". However, as scholars from both waves suggest, diaspora's analytic value comes from how it has changed the way that people think of nation-states and nationalities (Hall, 2003; Brubaker, 2005). Brubaker (2005, p. 13) states, “One of the virtues of 'diaspora', scholars have suggested, is that it provides an alternative to teleological, nationstatist understandings of immigration and assimilation". Nations are not created or constructed in binary ways, but are much more complex with globalization (Brubaker, 2005). Diaspora as a process illuminates non-binary, multiple definitions such as these (Dufoix, 2008).

The second-wave conceptualization of diaspora is also used to understand hybridity and theorize power, particularly colonial power (Bhabha, 1994; Hall, 2003). Diaspora identifies transnational practices, flows, and breaks down binaries of nationalism (Braziel, 2003). Brubaker (2005, p. 13) even describes that a critical approach to diaspora comes from "treating it as a category of practice, project, claim and stance, rather than as a bounded group". Diaspora as a process breaks down categories, colonial notions of purity, and also intersects with power relations and agency (Dickinson \& Bailey, 2007; Radhakrishnan, 2003).

Diaspora is complex precisely because it intersects with power, identity, agency, and has postcolonial implications. Not all diasporas are the same and have been affected differently by colonial and postcolonial migration (Dickinson and Bailey, 2007). For example, as Dickinson and Bailey (2007) suggest, the forced colonial migrants of India have fewer citizenship privileges than postcolonial migrants. In some ways, transnational processes in diasporas can deterritorialize creating a sense of "flexible citizenship" (Blunt, 
2007; Braziel, 2008; Brunea, 2010; Faist, 2010). But, they also re-territorialize by rearticulating specific territories or places or denying citizenship to certain groups or communities (Grewal \& Kaplan, 1994). Context is crucial. Some diasporas have limited mobility and cannot travel (Dickinson and Bailey, 2007). Limited mobility is true for not only larger diasporas, but also parts of diasporas (Dickinson and Bailey, 2007). The Indian diaspora's colonial and postcolonial migrants experience different types of mobility, especially regarding citizenship (Dickinson and Bailey, 2007) Academics need to address the diversity within diasporic communities precisely because two parts of the same diaspora can have very different experiences (Dickinson and Bailey, 2007).

Second-wave diaspora studies also illuminate hybrid and fluid identities (Hall, 2003). Identities are often fluid and not fixed. Sharma (2006) outlines this through an example of Bhangra, a product of hybridity. The Punjabi diaspora in Britain created Bhangra, a fusion of music from India and the West, but it spread to India. It now influences much music in India. In other words, the origins were not clear cut or singular. According to Gilroy (2003), the notion that a place that has no singular origins is key to diaspora.

Authors like Gilroy (1993) and Hartmann (2008) challenge binaries discussing how people engage with feeling. Through feeling and emotion, they experience identity, hybridity, and fluidity. Yet, feeling is also situated within a context and history of power. Gilroy (1993) suggests that people have found replacements for outdated notions of race, but these markers also carry similar othering and divisive implications. He suggests that this comes in part by representing fixed, single origins. This can be problematic, especially when identity, culture, diaspora, or life is rarely experienced in that way. Gilroy (1993) especially, contests notions of fixed origins and essentialist categories. He views culture, 
like identity and diaspora, as a process, not a category. The opening paragraph of The Black Atlantic describes the complexities of identity and diaspora:

Striving to be both European and black requires some specific form of doubleconsciousness. By saying this I do not mean to suggest that taking on either or both of these unfinished identities necessarily exhausts the subjective resources of any particular individual. However, where racist, nationalist, or ethnically absolutist discourses orchestrate political relationships so that these identities appear to be mutually exclusive, occupying the space between them or trying to demonstrate their continuity has been viewed as a provocative and even oppositional act of political insubordination" (Gilroy, 1993, p. 1)

First, like many identities, diasporic identities are unfinished and in a constant process of reformulation. They are also a 'double-consciousness,' a term that Gilroy (1993) borrows from W.E.B. Dubois. They include multiple intersections. Finally, as Bhabha (1994) theorizes, they are hybrid and "in-between". The "in-between" he refers to are the binaries associated with Orientalism and colonial power. Hartmann (2008) elaborates further. She brings in the emotionally-laden, lived aspect of diaspora, often missed in diaspora studies, but overlaps with the goals of NRTs. Hartman's (2008) goal is not to create a comprehensive overview of the "African-American diaspora". Rather, she creates a narrative based on emotion and experience that questions boundaries. She underscores the idea of "lose your mother" as a metaphor for losing your history, and in a sense, your identity and belonging: 
It is only when you are stranded in a hostile country that you need a romance of origins; it is only when you lose your mother that she becomes a myth; it is only when you fear the dislocation of the new that the old ways become precious, imperiled, and what your great-great-grandchildren will one day describe as African (Hartmann, 2008, p. 98).

Much like Gilroy's (1993) description of origins, Hartmann (2008) also sees origins as not fixed. There are no fixed places, spaces or origins, because they have been influenced by colonialism and become hybrid. For example, Hall (2003) suggests that the concept of an "original Africa" or an original anything, does not exist. Because we live in a dynamic, fluid world, an original reference point is unrealistic. Everyone has some "doubleness" or multipleness that does not fit fixed categories. Ifekwunigwe (2003) also highlights the complexity of diaspora in this way. She discusses it as an ongoing process, one that varies from person to person. While there is a sense of collective, imagined identity that ties people together, this identity changes by individual. Yet people are still affected by notions of origins. Even if origins and binaries do not exist, people still think about identities in such ways. In many ways, this is a product of hybridity. Hall (2003) suggests that while hybridity dismantles colonial purities, they continue to exist and displace identities. Thus, the critique that Hall (2003) and Gilroy (1994) make regarding origins, is in regard to dismantling colonial purity to stress the importance of hybrid identities. But, hybridity itself is problematic. Binaries and hyphens, as Mishra (1996a) suggests, imply an impure, problematic identity. For example, Indian-American, to Mishra (1996), implies that one is 
not fully American or Indian, but is impure American, and impure Indian. Grewal and Kaplan (1994, p. 7) describe this issue suggesting

...articulations of hybridity can be read to argue that Western culture is not pure, is not the origin or the destination of everything. Yet, what seems to get theorized in the West as "hybridity" remains enmeshed in the gaze of the West; Westerners see themselves alone as the ones that sort, differentiate, travel among, and become attached or attracted to the communities constituted by diasporas of human beings and the trade of commodities. Western culture continues to acknowledge difference primarily by differentiating the "exotic" from the "domestic"

In this way, hybridity and origins exist, but are often defined by the West, thus continue to be affected by colonial mindset, political agendas, and homogenization of identities into neat categories by governments, academics, and popular cultures. For scholars like (Ifekwunigwe, 2003), these binaries simultaneously creates days where origins are important and days, when they are not. She (2003, p. 196) describes:

On an empowered day, I describe myself as a diaspora(s) daughter with multiple migratory and ancestral reference points in Nigeria, Ireland, England, Guyana, and the United States. On a disempowered day, I am a nationless nomad who wanders from destination to destination in search of a singular site to name as home. 
These experiences are hybrid and "in-between" (Bhabha, 1994; Hall, 2003), but simultaneously affected by origins (Ifekwunigwe, 2003). Since 1994, the concept of hybridity has evolved. But, even scholars like Bhabha (2015) have critiqued scholarly applications of hybridity for missing the importance of locality and scale. While many scholars use hybridity to question origins, other scholars have discussed how place and scale are incredibly important to identity (Bose, 2018; Kaplan \& Herb, 2018).

\section{Scale and Diasporic Identity}

Scale has been important to geographic understanding of identity (Herb, 2018; Johnson \& Coleman, 2012; Kaplan, 2018; Nicley, 2009; Paasi, 2003) and to conversations on hybridity (Bhabha, 2015). Scholars have challenged the academic use of hybridity, noting that it is always defined by the West and homogenizes and "erases" differences between scalar identities, thus silencing the origins that make up the hybrid and inbetween (Bhabha, 2015; Papastergiadis, 2015). Bhabha (2015) has critiqued hybridity in part, because it overlooks the role of scale - especially in regard to local impacts on identities. Scale is central to geography but has received less attention in other disciplines (Culcasi, 2011). Geographers have identified scale as a means to mobilize counternarratives in communities. These, like hybridity, also "challenge(s) the hegemonic identity narratives" of nation-states (Paasi, 2003, p. 476). Scale has provided insights on how people use specific scalar (regional or local), often non-dominant identities to actively speak out against national hegemony (Johnson \& Coleman, 2012).

Scale is not a static, singular concept. Geographers emphasize scale's importance to movements against hegemonic identity. But, geographers also recognize that scale is 
contested and can be employed in multiple ways. Many emphasize that scale is sociallyconstructed, not fixed, emergent, not permanent, not pre-existing, and develops "at the moment" (Ferber \& Harris, 2013, p. 190; Marston, 2000). Marston (2000), for example, rejected scale in the traditional hierarchical sense, contributing to the wave of "flat" ontologies (Häkli, 2018). Flat ontological views of scale suggested that scales were not topdown, but rather flat and horizontal, meaning that hierarchical scales like global, national, regional, local were irrelevant (Häkli, 2018). But, some scholars interpreted scales such as national, regional, or diasporic not as fixed or essential, but instead as inherited (Häkli, 2018). In other words, they suggested that these scales can be mobilized during specific moments to dismantle national, homogenizing accounts of complex identities (Culcasi, 2011; Häkli, 2018; Johnson \& Coleman, 2012; Mackinnon, 2011; Paasi, 2003).

Scale, is inherently political (Johnson \& Coleman, 2012; Smith, 1996). Specific scales can be enacted for resistance and identity movements to highlight what is erased by dominant scales (Brenner, 2004; Johnson \& Coleman, 2012). For example, Johnson and Coleman (2012) show how regional identities create national identities. They argue that regional identities provide examples of negative or positive identities in a national context. For example, the authors show that Southern Italy or Eastern Germany were painted as "backward" and prohibitive of "progress". Meanwhile, Northern Italy and Western Germany were deemed as prime examples of ideal national identity. As the authors (2012) suggest, regional and national identity exist simultaneously and cannot be separated. These scales do not exist inherently but instead are created in the moment for a specific purpose (Brenner, 2004). 
How scales are enacted by certain groups, governments, and even academics are driven by complex power relations (Brenner, 2004). Power relations are embedded in everyday life in many ways (Brenner 2004). Power can be hierarchical, but can also be horizontal and flat (Brenner, 2004). People use scales, such as regional, or national depending on moment and context to navigate complex power relations (Brenner, 2004; Marston et al., 2009). Referring back to Johnson and Coleman (2012), people in Southern Italy used regional identities to counter-narrate dominant national identities. Yet, as the authors also suggest national identity relies on the existence of regional identity. For example, in their case study, governments and media often defined Italian national identity by defining it against the "backwardness" of Southern Italian regional identities. Thus, national identity should not be thought of as greater or further up in a hierarchy of scale. Scale is representational in that governments and media define particular scales or promote narratives of national, regional, or local identities, imbuing them with specific characteristics (i.e. patriotic, backward, unifying). These scales are also emergent because they are enacted in everyday life. People use scales to narrate or define identities within specific contexts (i.e. identifying as Southern Italian when threatened by the hegemonic narratives of Italian national identity). Scale is emergent also in part because regions or nations (whatever the scale may be) are not always bounded by specific spaces (Brenner, 2004). In other words, borders can be fluid. In some moments, identities become more fixed as they are enacted or performed. In others, they can be disregarded or ignored in favor of other scalar identities. They can be simultaneously fluid, changing based on moment. 


\section{Upcoming analysis}

Scale is seminal to geography. While some geographers have debated its importance, it remains relevant as one way that people understand identities in everyday life and navigate complex power relations. People mobilize different scales of (sociallyconstructed) identities, such as regional or national, during specific moments to dismantle national, homogenizing accounts of their complex identities. Scale demonstrates how identities, often homogenized, are heterogenous but at times solidify. Hybridity, meanwhile, shows the complex, overlapping, and hyphenated nature of these heterogenous identities. Hybridity is a complex process that elucidates how identities come from multiple sources, and can be blurred and in-between, but most importantly brings to light that identities are often in flux because of the legacies of colonialism that depict identities as binary and fixed. I develop my analysis of scale and hybridity in Chapter 4, demonstrating that participant's identities are both scaled (often fixed within moments), and simultaneously hybrid in others.

Identities, (particularly of people with ties to formerly-colonized nations or nationstates), have been influenced by colonial discourses and binaries (like West/East, colonized/colonizer). Postcolonial concepts like hybridity, demonstrate that identities are often in-between and blurred binary categories, and also influenced by the legacy of colonialism. Identities are also tied to colonial representations of whiteness which pervade in US society and within Indian communities. Mimicry, or the concept that the colonized mimic the colonizer to obtain the privileges of whiteness, is key to understanding how some diaspora communities in the US attain statuses of privilege or discriminate against other minority communities. I demonstrate the links between postcolonialism and 
whiteness in Chapters 6 and 7 when I discuss discrimination and othering both within the US and Indian communities. I show how the Indian

Identities are represented by governments and media (as other or in binary form like East/West or colonizer/colonized), but simultaneously lived and experienced. Diaspora communities are part of a complex process that involves displacement, hybridity, and tenuous links to origins. These identities are emotionally-laden, linked to memory, and experienced through a variety of senses including sound.

Sounds play a strategic role in navigating, articulating, and defining identities within diaspora communities. Sounds can be both represented to define identities in specific ways through scale. In Chapter 6, I show how musical events like the Cleveland Thyagaraja Aradhana use specific music and language to define and represent events as South Indian or Tamil. Sounds are also part of a non-representational experience of emotions and memory. In Chapter 5, I will demonstrate how sounds are important to how people experience their identities. For example, environmental sounds, like the whistling of a tea kettle, show how identities are connected to memories. 


\section{Chapter 3: Case Study and Methods}

In this chapter, I outline my case study, methods, data collection, and process of coding for analysis. First, I provide an overview of the Indian diaspora's migration to the US. Second, I provide an overview of my specific case study. Third, I discuss methods - both those used and those originally proposed but altered during the research process. Fourth, I discuss data compilation, coding, and analysis. Finally, I conclude with a discussion on my positionality and its implications to the research.

The Indian Diaspora - complex and multi-faceted

The Indian diaspora has two "official waves" - pre-independence and postindependence. However, some scholars acknowledge four phases rather than two waves. These include: first, forced colonial migration; second, voluntary migration to "Western" countries; third, the 1980s migration to the Persian Gulf; fourth, the 1990s "brain drain to the United States" (Dufoix, 2008, p. 42). However, most Indian diaspora scholars in the United States conceptualize the Indian diaspora in the two official waves recognized by the Indian government - pre- and post-independence diasporas (Dickinson and Bailey, 2007; Skop, 2012). Scholars, who study the diaspora in the US, often discuss the Indian diaspora in a post-independence context because the majority of diaspora population includes voluntary migrants that relocated after 1965 (Bhatia, 2007; Skop, 2012).

My dissertation focuses on the US diaspora post-independence, but it is important to note that both waves of the Indian diaspora are linked to the colonial past and have been 
affected by the violence of colonialism. The first wave encountered more direct colonial violence while the second experienced indirect violence, resulting from the aftermath of colonialization (Ray \& Mishra, 2009). The first wave of the Indian diaspora emerged from the colonial system of indentured labor in the 18th and 19th centuries - first, from Calcutta to Mauritius and soon to Fiji, Guyana, and other British 'sugar colonies' (Ray \& Mishra, 2009). This forced migration pattern changed after independence in 1947 and became "voluntary,"8 creating a second wave of migration (Ray \& Mishra, 2009). Now, with a diaspora of more than 20 million, it is the largest diaspora in the world with the fastest growing populations in North America (Dufoix, 2008; HLCID, 2001; Skop, 2012; UN, 2015). The Indian government's Report of the High Level Committee on the Indian Diaspora (2001) recognizes these two waves of diaspora, but generally discusses them as a singular entity. It acknowledges both the initial wave of forced migration from colonial rule and the second wave of voluntary migration. It also outlines the diaspora based on region and country of migration. The Indian government describes these diasporas a single diaspora at times yet enforces different policies for each wave (Dickinson and Bailey, 2007). It gives post-independence members some citizenship privileges but denies them for pre-independence members (Dickinson and Bailey, 2007). The voluntary wave of diaspora often has greater economic advantages and resources, thus is more valuable for return migration or visitation (Dickinson and Bailey, 2007). While the Indian government argues that citizenship privileges relate to India's independence and status as an official nation, Dickinson and Bailey (2007) argue that the citizenship privileges relate to economic

\footnotetext{
${ }^{8}$ Critical migration literature would question the voluntary nature of this migration.
} 
value as those in the second wave often have more money and wealth. Regardless of how these waves are categorized, it is evident that there are two very different diasporas.

They also differ with regard to origins as Cohen (1997) and Tölölyan (1996) discuss when creating typologies of diasporas. For example, pre-independence India and postindependence India would be two different Indias, and thus two different diasporas - one that existed as separate territories under British rule, and the other as a united nationstate. Like with many postcolonial nations, colonialism's influence complicated origins and categories as it shifted and changed many borders and boundaries around the world (Gilroy, 1993; Harris, 2014; Loomba, 1998; Mishra, 1996a; Sidaway, 2012; Sparke, 1998).

\section{Indian diaspora in the US}

Safran and Sahoo (2008) point out that the Indian diaspora, overall, is often classified as a larger, dispersed category across several nations. North America holds the fastest growing Indian population (Bhardwaj \& Rao, 1990; HLCID, 2001). The 1965 US immigration laws allowed increased migration from Asia, Africa, and the Middle East and the total Asian Indian population has almost doubled from 2000 to 2016, with close to 4.1 million living in the United States (Barrett, 2018; Bhardwaj \& Rao, 1990; Hoeffel, Rastogi, Kim, \& Shahid, 2012; Skop \& Li, 2005). This law allowed more Indians to come to the US for employment-based programs (Bhardwaj \& Rao, 1990; Skop \& Adams, 2009; Skop \& Li, 2005). Prior to 1965, Indian immigrants generally fell under broad categories like "Hindoo" and eventually "Hindu," despite religious affiliation (Bhardwaj \& Rao, 1990; Pew, 2015). In fact, the first Indian immigrants to the US were Punjabi settlers, often Sikhs, who provided 
agricultural support in California and worked the Western Pacific Railroad (Bhardwaj and Rao, 1990).

Punjabi settlers in California came as early as 1820 before India existed as a nationstate (Bhardwaj and Rao, 1990). Bhardwaj and Rao (1990, p. 198) highlight, "By 1910, this number (of Indian immigrants) was deemed alarming enough that the Asiatic Exclusion League and the American Federation of Labor began to brand the immigrant Indians variously as a 'Tide of Turbans', 'ragheads' and even a distinct 'menace'”. The Asiatic Exclusion League was specifically founded to stop migration from Asia. Anti-Asian sentiments beginning in the 1800 s and set the tone for views of Asians for much of the $20^{\text {th }}$ century (Eck, 2018). These sentiments were embraced by the Asiatic Exclusion League and also advanced by the 1917 Immigration Act that created the "Asiatic Barred Zone", prohibiting immigration from much of Asia including India (Bhardwaj \& Rao, 1990; Eck, 2018; Gibson, 1988). The 1924 Immigration Act placed quotas on the number of immigrants from Asia and was not repealed until the 1965 Immigration Act which gave preference to immigrants based on skills and family ties ${ }^{9}$ (Bhardwaj \& Rao, 1990; Chishti, Hipsman, \& Ball, 2015; Eck, 2018).

The US Census has categorized Indians in many ways since 1820, often shifting with immigration laws. Until 1980, Indian migrants to the United States were initially grouped with the original Punjabi settlers. Eventually, they were differentiated into an "Asian Indian" category after the 1980 rewrite of the Census categories (Pew, 2015). The most recent boom was in in the post-2000 era. The total Asian Indian population increased from

9 Though, it is also important to acknowledge that this bill was problematic for countries in the Americas in that it created conditions for "illegal" immigration from Mexico and other South and Central American countries (Massey \& Pren, 2012). 
$1,899,599$ in 2000 to 3,183,063 in 2010 and had the largest group in 23 states, most within the Midwestern, Southern and Eastern regions of the United States (Hoeffel et al., 2012). Many "Asian Indian" immigrants in the United States are highly skilled and migrate through securing jobs, educational opportunities or family sponsorship (Skop, 2012). This is in part due to the migration requirements and changes from the 1965 immigration laws, allowing migrants based on skill and family ties. According to the US 2010 Census, the Asian Indian subgroup has rates of $91.1 \%$ for high school completion and $70.7 \%$ for Bachelor's degree or higher education (Hoeffel et al., 2012). While this is the official data on the Indian diaspora, many organizations have estimated that Indians, and Asians in general, are becoming one of the largest group of undocumented immigrants in the United States (Passel \& Cohn, 2016, 2017; Sridaran et al., 2017)

\section{Indian Tamil Diaspora in the US}

The Indian diaspora is fractured at more than two divisions of pre- and postindependence India. Many scholars have referred to subdivisions of the diaspora along religious, regional, linguistic, and cultural lines as "diasporas within a diaspora" and "subdiasporas" (Bhardwaj \& Rao, 1990; Bhatia, 2007; Dufoix, 2008; Safran et al., 2008). Scholars such as Sahoo (2006) discuss the importance of regional divisions of the Gujarati diaspora within the Indian diaspora. Voigt-Graf (2004), though examining transnational networks, divides the diaspora on state or city location. Safran, Sahoo, and Lal (2008) argue that subregional focus is important precisely because homogenization masks various nuances of religions, languages, and other subregional divisions of the Indian diaspora. The 
Indian Tamil diaspora that I examine comes from the Indian state of Tamil Nadu (see Figure 1).

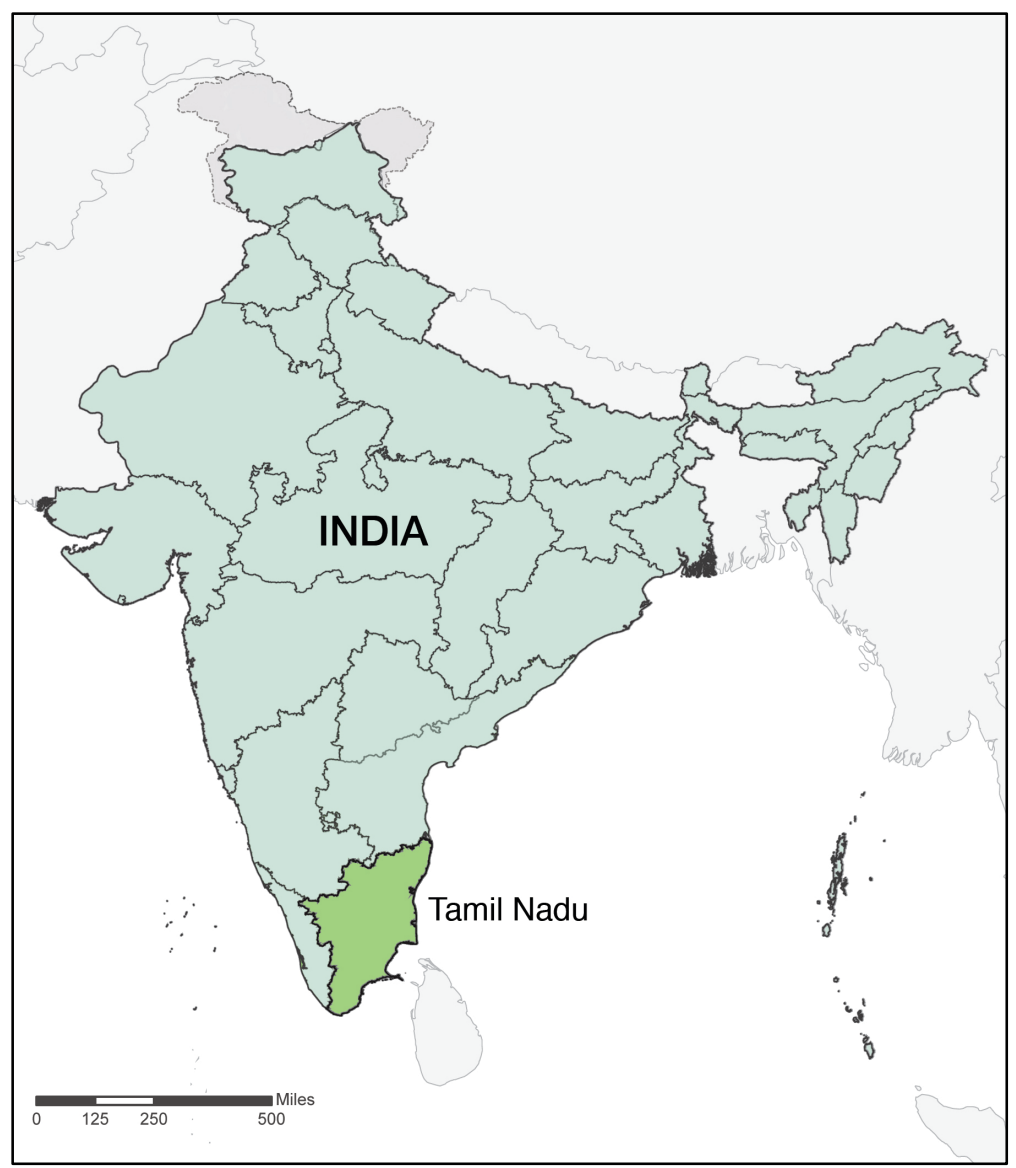

Figure 1: State of Tamil Nadu

Because the diverse Indian diaspora is often treated as a single, unified entity, those with origins in Tamil Nadu are often masked by the broad category of "Asian Indian". Regional identities as outlined by Bhardwaj and Rao (1990), Voigt-graf (2004), and Safran, Sahoo, and Lal (2008) are important to research on the Indian diaspora because academics and governments often homogenize the Indian diaspora. Research on Sri Lankan Tamil exile communities abroad exists, but studies on the Indian Tamil community specifically are sparse. Muddying an already complex diaspora, many Indian Tamil community organizations in the United States also incorporate Tamils from Sri Lanka. Overlap between 
Indian and Sri Lankan Tamils occurs in social circles, but distinction based on nation-state boundaries is still quite visible. Sri Lankan Tamil communities often have their own separate social organizations. Worldwide, there are approximately 70 million Tamil speakers in 50 countries with local Sangams, Tamil cultural organizations, for each community (Bhat \& Narayan, 2010). In the United States, there are three to four Sangams per state where nearly 131,000 Tamil speakers reside (Hoeffel et al., 2012).

Sangams, particularly those associated with Tamil Nadu in India, promote a Tamil anthem, attire, and flag that emphasize cultural elements of Sangam Age, yet often situate these within the context of India (Bhat and Narayan 2010). According to former Tamil Sangam president, the Northeast Ohio Tamil Sangam represents approximately 110 "official" families and 25-35 nonmember families from varying religious backgrounds, though the majority are Hindu. Often, members purchase one membership for the family or attend events without a membership. Members come from Chennai, Madurai, Coimbatore, Karaikudi, Nagercoil, Tiruchirappalli; other states of India such as Maharashtra, West Bengal, or Karnataka; and even countries including Malaysia and Sri Lanka. Despite having members from other countries, Sangam events specifically focus on India and Tamil Nadu.

\section{Research Sites}

I based my interview and participant observation on three sites - Northeast Ohio, northern New Jersey, and Morgantown, WV. I emphasized Northeast $\mathrm{OH}$ as a primary site for data collection but benefited from a multi-site analysis including northern New Jersey and Morgantown, WV. Cleveland, $\mathrm{OH}$ provides a significant "non-traditional gateway community" for Indian Tamils. According to the definition provided by Skop (2012), a non- 
traditional gateway community serves as one that is not the largest by population and physical size but has a population compared to other communities in the US and thus provides a lens that is generalizable to other communities in the United States. Skop (2012) and Skop and Li (2005) advocate for examining these non-traditional gateways that are often medium-size and reflect broader settlement patterns of Asian Indians. Nontraditional gateway communities like Cleveland are not outliers compared to many other cities in the United States and therefore provide key samples of diasporic communities that can easily be applied to other cities in the US (Skop, 2012). Cleveland, OH also hosts the Cleveland Thyagaraja Aradhana, the second largest festival of South Indian classical music in the world every year, bringing together Indian Tamils from all over the world (CTA, n.d.; Viswanathan \& Allen, 2004). South India usually refers to India's southern states of Tamil Nadu, Andhra Pradesh, Kerala, Karnataka, and more recently, Telangana. 


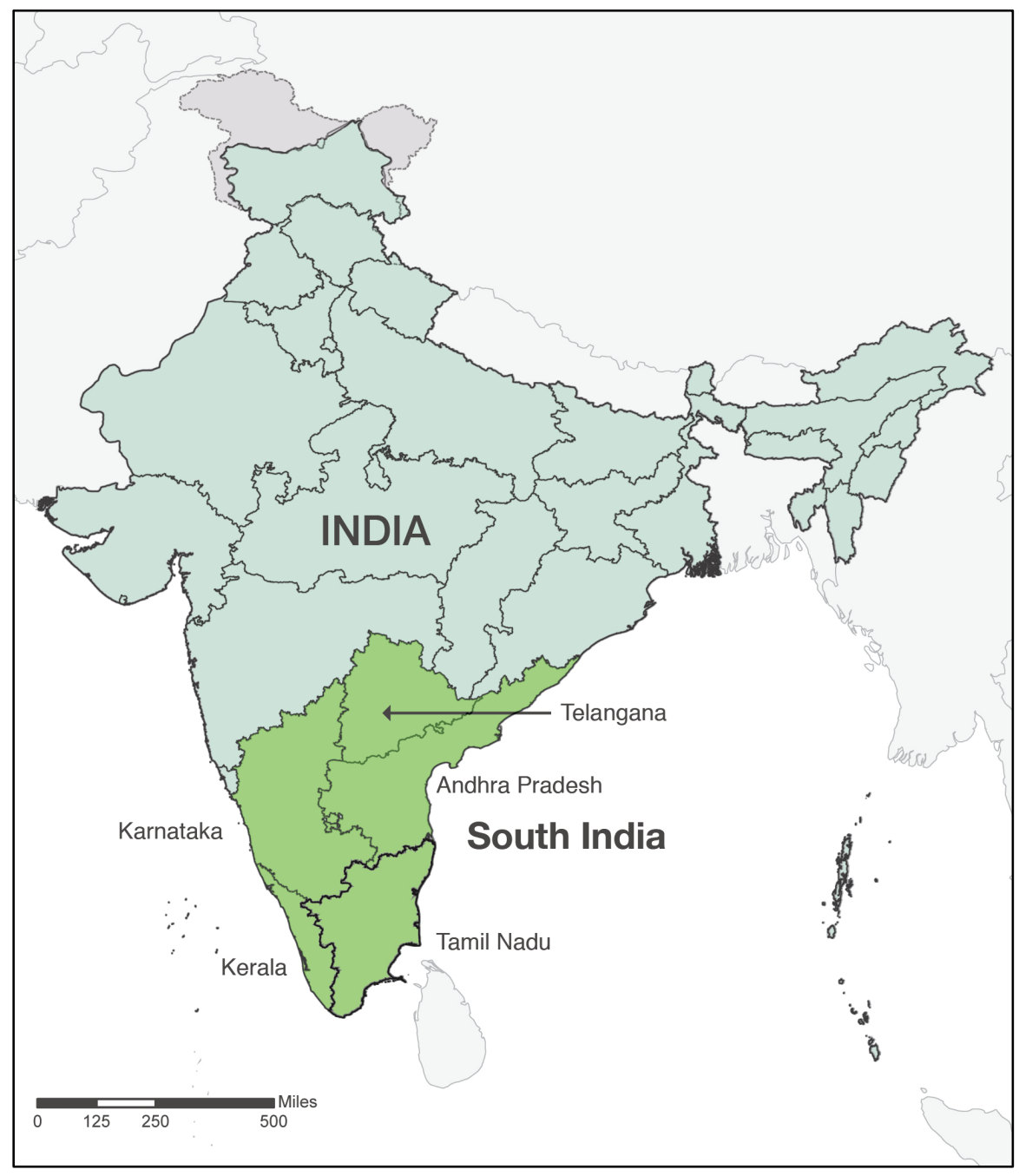

Figure 2: States of South India

It was important to contextualize my research with three differing sites.

Morgantown, WV is the smallest of the three and is the least-connected area in terms of Indian Tamil communities. For example, many participants I interviewed from this area said that there were not as many Tamils, so they attended broader "Indian" events or had more Indian friends. They described that they had to travel to Pittsburgh or other cities to attend more specific “Tamil events." Cleveland was larger than Morgantown, but smaller than New Jersey. It had a significant Tamil population, enough to have events, schools, and other programs, but was not an outlier like New Jersey. New Jersey has the largest 
concentration of Indian Tamils in the US as well as the largest Tamil association (NJTS, 2017; Zong \& Batalova, 2015). New Jersey had multiple sites, events, performances, and even communities. For my New Jersey research, I traveled to many areas in northern New Jersey, including Edison, Trenton, and suburbs. The New Jersey Tamil Sangam claims to be the largest in the US. This is important, but as mentioned by many participants, Tamil communities in places like New Jersey have resources unlike most other areas with Tamils in the US.

The bulk of my participant observation data came from Cleveland. I did this for three reasons. First, it is a non-traditional gateway community and is thus more applicable to other Tamil communities in the US (Skop, 2012). Additionally, much research already focuses on large communities like New Jersey, marginalizing Skop's (2012) definition of "non-traditional gateway" communities. Second, I had already established connections from previous research that allowed me to gain significant access to events and programs. Third, I was able to maintain consistent presence in the community instead of sporadically attending events or attending them as someone's guest as I did in Morgantown and New Jersey.

Although Cleveland and Northeast Ohio served as the basis for participant observation, interviews were connected to each site including northern New Jersey and Morgantown, West Virginia. The majority of my interviews came from connections to New Jersey and Northeast Ohio. I had fewer participants with connections to Morgantown, WV. But, as many participants mentioned and through my observation, Morgantown's Tamil community is much smaller than Cleveland or New Jersey. US census data does not identify subsets of the Indian diaspora and to revisit Bose's $(2018,262)$ description, "is a poor way 
of aligning the various parts of the diasporic identity - region language, ethnicity, and culture being a few of these." Additionally, identifying Tamil populations through language is also difficult as Tamil is spoken in more than India. Also, participants who identify as Tamil or participated in Tamil communities did not always speak Tamil. Thus, much of my information on Tamil community sizes came from observation and/or Tamil Sangam statistics.

\section{Methods}

My research relies on two main methods - in-depth interviews and participant observation. I recruited interviews in three ways. First, through snowball sampling, asking participants to identify other participants or have them share my recruitment letter (See Appendix C). In communities not necessarily recognized by official census data such as the Indian Tamil community, snowball sampling is the best way to identify members (Dunn, 2010b). Prior to my doctoral research, I had already spent three years involved in the community in Cleveland and developed several contacts.

Second, I asked community leaders/facilitators including presidents of Indian diaspora societies or organizers of events to participate and also identify other potential participants. My interviews in New Jersey often came from suggestions of contacts in Cleveland and I used snowball sampling after subsequently developing contacts in New Jersey. Finally, I recruited interviews when attending events during participant observation. I asked people I met at events or performances if they would be interested in participating in an interview. My interviews in Cleveland and Morgantown came from attending events and through snowball sampling. Interviews included individuals from two 
generations (all from the second wave of the Indian diaspora) - first and second - that identified as members of the Indian Tamil diaspora. I selected participants based on if they identified as Indian Tamil and lived in the United States. Interviews allowed people to discuss their identities in detail and depth. Interviews were connected to three specific areas - Northeast Ohio, Morgantown, West Virginia, and northern New Jersey. By connected to, I mean that participants had either lived in those areas at one point in their lives or had family who lived those areas.

Interviews are useful to gauge complex processes of identity and to contextualize observational data (Dunn, 2010a). Interviews identify what is relevant to interviewees, highlight multiple interpretations of meanings, give voice to people, and fill gaps in other data collection methods like participant observation (Dunn, 2010a; Tacchi, Slater, \& Hearn, 2003). Additionally, interviews with participants are important to establish perspectives beyond dominant narratives of film, media, or official representations, drawing attention to people's everyday experiences as NRTs suggest (Dunn, 2010a). My interviews lasted anywhere from 35 minutes to 3.5 hours. Most interviews averaged around 1.5 hours. I used an audio-recording device to record each of my interviews and wrote detailed notes, transcribing as participants spoke. I conducted some interviews in person and some over the phone, depending on the participant's availability. There was one instance that I had to conduct an interview through email because the participant was hearing-impaired and thus preferred to communicate via written text.

I conducted 58 interviews total. I removed five from the analysis after I found that they did not fit the initial criteria of identifying as Indian Tamil. Participants had identified as Tamil, but I found out during the interview that they identified as Sri Lankan Tamil, not 
Indian Tamil ${ }^{10}$. Out of the remaining 53, I used 39 as in-depth interviews in the analysis. The other 14 provided information described as "conversational interviews," but did not maintain the consistency of the other 39. In other words, these participants never completed the full interview, or only had time for part of the interview and thus, these interviews did not fit the consistency standard that I used to code in NVivo (discussed in subsequent section). I still considered these interviews valuable but did not feel that they should be classified in the same way as the other full 39 interviews.

Interview questions revolved around identity (See Appendix A). Crafted after Dunn's (2010) model, I relied on multiple types of questions. Descriptive questions included details about "events, places, people, and experiences." Storytelling questions allowed more personal input. Opinion-based questions gathered dialogue on feelings, impressions, or value judgments (Dunn 2010b, p. 106) (see Appendix A). I also included a question that allowed participants to discuss any other items that they felt were important. After introductions, I asked participants to describe their identities using at least 5 words. I then asked them to tell me what each identity meant to them personally. Afterward, I asked what qualities or characteristics they associated with these identities. I asked more specifically about Indian, Tamil, or American identities, even if these were not mentioned, as identifying as Indian and Tamil was required to participate in the interview.

After establishing a baseline for identity, I asked participants about sound. First, I asked them if they associated any sounds with any of the identities that they mentioned. Later, I asked them to rate the importance of sound to their identities. Finally, I asked them broadly about discrimination and if they had ever felt discriminated against or

${ }^{10}$ These interviews did provide preliminary data for future research. 
marginalized within the US or Indian communities. I also asked participants if they had ever felt discriminated against or marginalized regarding music, accent, language, or any other sounds.

I am familiar with Tamil but conducted interviews in English as it is often the preferred language for those in the second generation and for some in the immigrant generation of Indian Tamils in the US ${ }^{11}$. Also, English is a national language of India, used often in many parts of South India over Hindi, India's other national language. Occasionally, if participants referenced Tamil or Sanskrit words (See chapters 5-8). I gave all participants pseudonyms to protect their identities. In some cases, I chose to remove specific location information from interviews to further protect anonymity.

\section{Participant Observation}

Observational research is an active way to develop complementary evidence for contextualizing other methods such as interviews or surveys (Kearns, 2010). Participant observation, more specifically, has been used by many geographers in human geographic research because it allows geographers to uncover diversity and complexity of everyday life by observing and participating in events, functions, and communities and has been used quite frequently and successfully in many studies (Kearns 2010).

Participant observation was useful for understanding how communities, events, or performances represented or mobilized certain identities. I attended 20 Indian diaspora events over three years such as the Cleveland Thyagaraja Aradhana, Deepavali, Pongal, Chithirai Thiruvizha, and other Indian programs, each year. I attended a Tamil school in

${ }^{11}$ Especially when speaking to someone like me whose Tamil vocabulary is limited. 
Northeast Ohio every Friday for a period of one year. I also attended multiple informal events and gatherings from community members.

Through these events/gatherings, I looked for multiple insights. First, how people and events engaged with music, accent, or language. Second, how scale or hybridity was threaded throughout programs. Finally, I looked for how performance, events, and programs represented identities. I examined printed materials such as programs or flyers, performances, facilitation, and general audience interaction. I recorded observations through field notes, videos, photographs, and audio recordings. I then compiled these into NVivo for analysis.

Researchers often document participant observation through field notes to identify important elements of the observational experience (Tacchi et al., 2003). Thus, field notes contained entries of observations and experiences that could add, affect, or prove important to the research. Included in these notes were references to sounds. First, I included sounds of music such as songs from popular films, classical music such as the Karnatak tradition, folk music. Second, I included sounds of language and accent - Tamil, Tamglish (mixture of Tamil and English), English, Hindi, Telugu, Sanskrit, Malayalam. Third, I included environmental sounds like anklet bells from the feet of Bharatanatyam dancers, clapping, silk sarees sweeping across the floor - that occur in the performances or at the events. At times, I photographed or recorded audio or video clips to further document observations and subsequently compiled these with field notes.

\section{Altered Methods}


In my initial proposal I planned on using two additional methods - Audiovoice ${ }^{12}$, similar to PhotoVoice, and a brief questionnaire. However, after beginning the research, I found that I was best able to answer my research questions through interviews and participant observation. I initially asked participants to partake in Audiovoice but found that it did not yield the data I needed. Participants did not participate consistently and often forgot to send the data. Additionally, most participants would send items that they enjoyed like YouTube videos or websites, but these items were often unrelated to the research project. Thus, I stopped asking for participation after the first 10 interviews.

With the brief questionnaire, I found multiple issues. The first issue was that most of my questionnaire questions were similar to my interview questions. Thus, I was repeating the data that I was already gathering. The second issue I found was that the questions I needed to ask were too complicated for a questionnaire. To obtain the data I was looking for, the questionnaire required long answers, much too long for a brief questionnaire. I best obtained these answers through interviews where I could elaborate and explain the nature of the questions. I did, however, ask participants to rate the importance of sound to identity on a Likert scale. Instead of including this on a questionnaire, I asked this after completing the first interview. In this way, participants already understood the nature and scope of the project and could effectively understand the terminology that I used. So, I kept one component of the questionnaire (See Appendix A), using it after interviews. Most participants who completed interviews, answered this question.

12 This is where I described having participants record sounds or clips that were relevant to their identities over a period of two weeks. 


\section{Data, Coding, and Analysis}

I analyzed the 39 interviews and my notes from my observations in a qualitative software analysis program called NVivo. NVivo allows linkages and coding between multiple types of data including audio, images, video, textual data, field notes, and transcribed interviews. I conducted an qualitative thematic analysis, first coding interviews cases that include demographic information such as age, gender, location (where participants had grown up and where they lived now), and generation. I then coded themes at broader and more specific nodes. For example, broader themes like "discrimination" include subcategories such as gender, caste, power relations, hegemony, skin color, othering, hate crimes, orientalism, Indian racism, pronunciation, 9/11, and Trump election that intersect with "sound" through subcategories of music, accent, language, lack of sound, environmental sounds (for a full list, see Appendix B). I focused on categories that related to spatial identities. For instance, I coded for "identity" to include "Indian”, “Tamil”, “American”, “South Indian”, “desi”, and "brown." Participants identified as Indian, Tamil, and American most often. I would inquire when needed to ensure that we discussed their sense of identity in detail. I asked that participants identify as Indian and Tamil or at least as part of the Indian Tamil community to participate in the interviews. In my analysis, I only included interviews from participants who identified as such. In some cases, participants identified as Indian or Tamil, but said that they did not always feel Indian or Tamil. I would then ask them to elaborate. Not all participants identified as American. Most identified as Tamil and Indian. Some would identify as Indian and not Tamil and vice versa. 
I used NVivo for both a coding and organizational tool. I used NVivo to create different category folders (like an organizational Desktop folder) for each type of data. For example, I included interviews in one folder and participant observation in another. Within the participant observation folder, I also included subfolders to separate the data based on audio, visuals, events, and other items. I coded each type of data. I used coding to begin the analysis, but later, it became more of a place marker and categories so that I could easily find my data. I did not fully transcribe interviews but took detailed notes during them and included time stamps. I included all the main points of the interviews with time stamps directing me to specific areas in the audio file for further transcription if necessary. For example, in the analysis, to pull a specific quote, I referred to a time stamp and used it to listen and double-check my transcription of that part of the interview. I coded according to themes and sub-themes that related to identity and sound (See Appendix B).

During my analysis, I used both code and word search queries to compile the data that I had on specific topics. For example, word search queries allowed me to look for all data that contained the word "Tamil." I was then able to examine all instances where notes or interviews used the word Tamil. But, while word search queries were helpful for initial examining of the data, I relied much more on queries using codes for analysis. For example, when examining sound, I searched for all items that I coded as related to sound. From there, I was able to look more specifically at music, accent, or language. I could also cross examine multiple areas of coded data. For example, if looking at discrimination and sound or more specifically discrimination and accent, I could search for areas where accent and discrimination intersected in my data. These queries would include transcribed interviews, 
scanned copies of event program materials, or images of events where I had added keyword descriptors.

I coded audio data separately. NVivo is not a good tool to code audio or video data. For video and audio data (like videos or audio recordings of events and performances), I was able to store these data in the same project file as the textual and visual data but was not able to analyze them in the same way. So, NVivo worked well for organization (i.e. a digital location in which to store multiple types of data like transcribed interviews or video recordings), but I had to complete my analysis separately using the "notes" and "memos" tools in NVivo. In other words, I had to take notes about what the audio and video contained. I used the NVivo note and memo functions to type notes directly in the project file. In general though, the bulk of my data came from my interviews and textual notes, so this was not a major issue. It did, however, drive me more toward traditional, textual dissemination of my analysis instead of a creative, sound-based presentation of my data, I analyzed my data looking for how my interviewees' senses of identity fit into hierarchal scales as well as transcending scale to more hybrid identities. At times, participants would use the terms Indian, Tamil, American, Indian-Tamil, Indian-American or local villages, cities, regions in Tamil Nadu and the US to describe some aspect of their identities. In analysis, I connected the concepts of hybridity and scale with participant's terminology. I coded identities with words that participants used like Tamil, Indian, South Asian, desi, Madurai, Coimbatore, specific villages, etc. I then classified these in the analysis as regional, national, supranational, subregional, local, and global, based on how participants framed these identities. I also coded them as hybrid, hybrid scales, or multiscaled (prior to analysis), again depending on context of the interview and how 
participants described or discussed their identities. Not all participants used terms that I use in my analysis, though some did, like whiteness, anti-immigrant sentiments, or Islamophobia. But, they effectively described such items using phrases like "fear of Muslims" or "afraid of immigrants" or "threatened by immigrants". I often used these particular terms to classify what participants said. I did not code in NVivo in the way that I did for concepts like identity, sound, and discrimination. I found that NVivo was more supportive of descriptive codes. I used the first codes, intersecting them, and then laying them out to develop conceptual codes that reflected these concepts also stored through "notes" or "memos". These "notes" and "memos" were used to inform my written analysis in Chapters 4-7. This allowed me greater flexibility in interacting with the data, while still allowing me to ground my analysis in the previous coding.

While I did record audio and visuals, I found that traditional academic analysis has many constraints. I had discussed in my proposal the possibility of including visuals and audio, but found that in general, this type of analysis is supported through textual presentation of data, such as that of a dissertation. Therefore, audio and video, while it could provide useful information, still needs to be presented in textual format through textual analysis. At some point in my career, I would like to work toward creating a more multi-sensory presentation of data and writing this dissertation has inspired me to work toward this goal in the future.

\section{Positionality}

Scholars have addressed issues of positionality and its effects on interviews and participant observation (Aitken, 2010; Besio, 2003; Myers, 2010). Dowling (2010) 
describes that recognizing positionality is important when discussing issues of emotions, power, and intersubjectivity because the researcher's background inextricably influences how the researcher sees and understands the data. Myers (2010) describes "representing others" as personal, but also having the danger of creating a "partial story." Every story needs balance - recognizing the researcher's position but also remaining "neutral" (Myers, 2010). Some scholars have pointed out that neutrality is often overemphasized, suggesting that "detachment, objectivity, and rationality" have been valued in research, but "engagement, subjectivity, passion, and desire" have been undervalued (Anderson \& Smith, 2001, p. 7; Nash, 2000; Smith, 2011; Thien, 2005; Tolia-Kelly, 2006). Concepts like "objectivity" or "rationality" often do not acknowledge bias and can create broad, sweeping generalizations of people (Tolia-Kelly 2006). Emotions, passion, and subjectivity can bring a more nuanced understanding of a situation but are often under-examined (Tolia-Kelly 2006).

I have a Tamil background and I am conducting research on others with a Tamil background. My positionality is important to recognize as the stories that I share in my dissertation closely resemble my own personal experiences. Scholars describe how emotions affect how researchers situate themselves and their understandings in research (Aitken, 2010). Emotions are integral parts of encounters, both with the researcher and those being researched (Aitken, 2010). It would be unethical to pretend that I do not have some emotional investment in this project. Likewise, it would be unethical to not point out that while interviewees shared these stories, I also felt emotional at times. There were many instances I felt deep emotion, especially when participants described the struggle of their own identities, instances where they were othered or discriminated against, or even 
the heartbreak of feeling rejected in their own country after the recent election. I cannot pretend that I have not felt similar or described similar experiences.

I recognize that my position as someone with a Tamil background indeed influences the way that I view my own research. Yet, I also recognize that it was my position that inspired me to conduct this research in the first place. For example, it allowed me to relate to participants with particular topics like discrimination or even experiences of growing up with Tamil family. I was able to communicate with many, especially second generation participants, on topics that someone who did not share similar experiences might not be able to relate. Some participants had even asked me if I had experienced issues relating to discrimination that they described. I chose to share these stories when asked and thus, found that I was in a process of exchanging stories with other participants. Two participants actually asked for the interview recordings for their personal use. One participant even interviewed me simultaneously as I interviewed them. They asked me questions for their own personal research project on minority experiences in the US.

Though I began this research 5-6 years ago during my MA work, my interest in identity has been life-long. I have struggled with some of the issues that participants described. Being a part of the Indian diaspora means that I'm American, but yet because of I am also not American in the same way that some of my white friends were American. Some days I felt that I lived two, sometimes multiple, different lives. As the daughter of Indian Tamil immigrant and a white American mother, I struggled to occupy these multiple identities of South Indian, Tamil Indian, Indian diaspora, among others. In some moments, I think of my identity as quite hybrid - I think of myself as a blurred Indian-American. I also attribute my hybrid, in-betweenness to struggling to fit into the Indian/American or 
east/West binaries. Categories that suggest that Indian and American are two separate, distinct identities and being American means that I have to be white. Thus, I am inbetween.

Through conducting this research, I also realized that like many participants, I also at times, conceptualized my identities, depending on situation and context, in a very hierarchical scalar way. As an academic, I often emphasize that identity is fluid, but realized that in my own personal life, there are many moments that I think about identity as hierarchically scaled and describe it as fixed at times. I do not think about it as fixed all of the time, but like Marston (2000) describes, this notion of scale emerges in specific moments. I too, like many participants find that I describe my identity in a very hierarchical way, depending on situation. Much like participants described, if I'm talking to other Indians, I make note that my family is Tamil. If I'm talking to Americans, I describe my identity as Indian. In some circumstances, I will also say South Asian. When talking to other Tamils, I describe my family's identity as from Chennai. Though, in other circumstances, I do make note that my grandmother was from Vellore and my grandfather from Pudukkottai - two very distinct and different areas of Tamil Nadu. It really depends on the audience, situation, and context. Identity is never fixed yet stabilizes in moments even in my own experiences. This project influenced me to deconstruct my own position and identity, analyzing it in ways that I had not done before like through scale, hybridity, and sound.

Initially in this research, I did not intend to discuss issues of discrimination or marginalization in current political climates. Yet, it continuously surfaced in my research and interviews. I should also acknowledge my position on this issue as well. I grew up in a 
very rural part of Ohio in a town of about 800 other people. My father, brother, and I were the only non-white people in this town. My family experienced a significant amount of racism and anti-immigration sentiments, which increased after 9/11. My dad was Tamil, but also a devout Indian, who would never give up his Indian citizenship. It was a source of concern for my family, especially after 9/11. It is a recurring concern that surfaced again with the Trump administration. My father, like many other participants I spoke with, was a product of "chain migration" that the Trump administration continuously attacks. He is afraid to leave the country, fearing that he won't be let back in the US with a green card. When we talk on the phone or in person, he tells me stories of other people he knows with green cards that didn't come back and were denied entry. We returned from India right before the Trump election, knowing fully that it might be years before my father can visit his home and family again. Throughout this research, I was reminded that many people are experiencing similar fears and situations.

The recent 2016 election reminded me of the harrowing implications of identity. I first realized this after 9/11, when suddenly, I knew that I would always be "brown." I was again reminded by the 2016 election, that there are people who actively want to eradicate "brown" or "non-white" or even "hybrid" people in America. Many second generation participants also described this sentiment and I could easily relate. Through this research, I developed friendships with a few participants. It is not my intention to leave this community or abandon it, as I am intricately connected to it in so many ways. 


\section{Analysis Ahead}

In the next four chapters, I share the stories of participants and my learnings from participant observations. I focus on their senses/experiences of identity in four ways. First, I demonstrate how scale and hybridity are important to understanding participant experiences with identities in Chapter 4. Second, in Chapter 5, I discuss sound's integral role in identity politics. Third, I analyze how discrimination and othering affect Indian diaspora experiences in US society in Chapter 6. Fourth, I analyze discrimination and othering through internal complexities of identity politics within Indian communities in Chapter 7. I examine specific experiences of individuals as well as broader community sentiments regarding identity politics. But before this analysis, in Chapter 4 I discuss in more detail scale and hybridity as these are the foundational concepts relevant to the remainder of my analysis. 


\section{Chapter 4-Hybridity and Scale}

When I talk to people in my village, they will be able to identify me and my family because of my name - we know your parents and grandparents. When I talk to my American friends, they think, this person is from India. Sometimes, they will ask me which part of India are you from? But they know little geography of India. - Raj

Raj, who lived most of his life in Tamil Nadu, recently came to the US. He identifies strongly not just with being Tamil, but with a specific village. His village, near Puducherry, TN, is crucial to his identity. Yet, now that Raj lives outside of his village, he says he is forced to use a broader identity. Local and global scales emerge in specific moments for individuals to claim either national or local identities (Antonsich, 2018). Raj is forced to use a national identity when talking to non-Indian Americans but identifies locally with his village when he talks with Tamils. At the same time, he lives in the US and participates in US society, which still maintains binary colonial categories such as that of Indian, thus his identity is also hybrid, blurred and informed by his life in the US. Local and global scales are important to identity, but also blur and are influenced by a specific history and context (Antonsich, 2018). Raj's local identity in Tamil Nadu also influences his experience in the US and in US society.

The main focus of this chapter is hybridity and scale and how both concepts work simultaneously in everyday experiences, representation, and performances of identity. This chapter highlights how participants navigate both scaled and hybrid identity in the US. Tensions between participant's described identities like Indian, Tamil, American, and 
others are highlighted through scale and hybridity. The implications of sound on hybridity and scale will be developed in Chapters 5 .

\section{Hybridity and Scale}

In Chapter 2, I discussed separately the importance of hybridity and scale. Most literature has not addressed hybridity and scale together, but rather as isolated, often competing or non-related concepts. Recently, however, some scholars have broadly examined the links between these two important ideas. Researchers regularly discuss that identities are hybrid, intersectional, and fluid. Identities often connect, intersect, and blur in moments, while maintaining connections to historical contexts, like in the case of the Indian diaspora and colonialism. Both hybridity and scale are important to situating multiple origins and scales of the diaspora to prevent homogenization or erasure of nondominant or non-hegemonic identities, while also situating them within a specific historical context.

Scale, like hybridity, is not concrete, but unlike hybridity, is not always hyphenated. Instead, it is more often used as a lens to view, understand, or mobilize particular issues or identities. Scale is essentially a way for humans to bind and navigate space (Herod and Wright 2002). Scale, in general, it is a part of a larger network, connected in various ways that humans mobilize to understand relationships (Herod \& Wright, 2002). In Chapter 2, I discussed how Marston (2000) critiques traditional scales for their hierarchical nature that create essentialized categories. The debate over scale continued as many scholars built on Marston's analysis and thus, the wave of "flat" ontologies that rejected scale became prevalent in geography (Häkli, 2018). Yet, as Häkli (2018) concludes, scale has not 
disappeared with these debates but remains relevant and crucial to geographic discussions, especially relating to identity. As Kaplan (2018, p. 31) suggests, it is difficult to understand identity "without reference to scale" as identity and scale are invariably linked. Scale is not only relevant, but most scholar acknowledge that it is not fixed and is quite complex (Johnson and Coleman, 2012; Culcasi, 2018; Häkli, 2018, Kaplan, 2018, Johnson, 2018). Much literature has rejected traditional scale, suggesting that it is merely sociallyconstructed, has no ontological reality, and thus is not useful to human geography (Marston, 2000; Häkli, 2018). Yet, Häkli (2018) and Kaplan (2018) have indicated, traditional, hierarchical scale is still relevant to identity. In fact, within my own research, I found that many people, in moments, do think about identity through traditional, hierarchical scales. While academics like Marston (2000) and others are valid in their critique of the dangers of hierarchical scale, these socially-constructed categories still manifest in moments in everyday life.

Traditional, hierarchical scales surfaced in most of my data and interviews, yet, these scales were simultaneously hybrid and emerging depending on situation and context. Identities were hybrid in multiple ways. The first way in which they were hybrid was that they were multi-scalar, existing as two scales simultaneously, or hybrid in that they were blurred. As Antonsich (2018) notes, identities are clearly fixed as global, local, (in my study also regional) in some moments, and simultaneously blurred and multiple in others. As Kaplan (2018) suggests, "We live in a multi-scalar and geographically complex world, in which identities manifest themselves in several different ways..." Identities manifested as hierarchical, but in other moments as hybrid, blurred, and multiple. However, hybridity existed in multiple ways, not just as a product of blurred identity. 
Identities were hybrid in a second way. They were hybrid because of their links to colonialism. In other words, the very condition of having ties to a postcolonial state created tensions between reinforced colonial binary identities. In other words, hybridity did not just imply that identities are blurred, but also indicated as Bhabha (1994) describes that they were "in-between." For Bhabha (1994) being "in-between" is a result of colonial imaginings of colonizer and colonized and anything in between that binary is not legitimized as valid. Scholars have also suggested that even in settler colonial states like the US, colonial binaries are tools that define "civility and savagery" (C. Harris, 2004, p. 165; Seth, 2010; Veracini, 2013). Simply put, colonialism depicts identities as pure and fixed, but hybridity and "in-betweenness" can be a threat to colonial societies because they dismantle this fixity. So, in some ways, the second way that identities were hybrid was also connected to the first in that both instances of hybridity imply blurred lines between identities. Yet, importantly, hybridity that is postcolonial derives from a specific historical context that can both reinforce and challenge colonialism.

In an effort to best illustrate how identities worked within the context of my study, I've created a chart that demonstrates the relationship between the elements. I have charted responses from my participants to question about identity. Those included in the chart are spatially-oriented common descriptors that surfaced throughout the interview. 


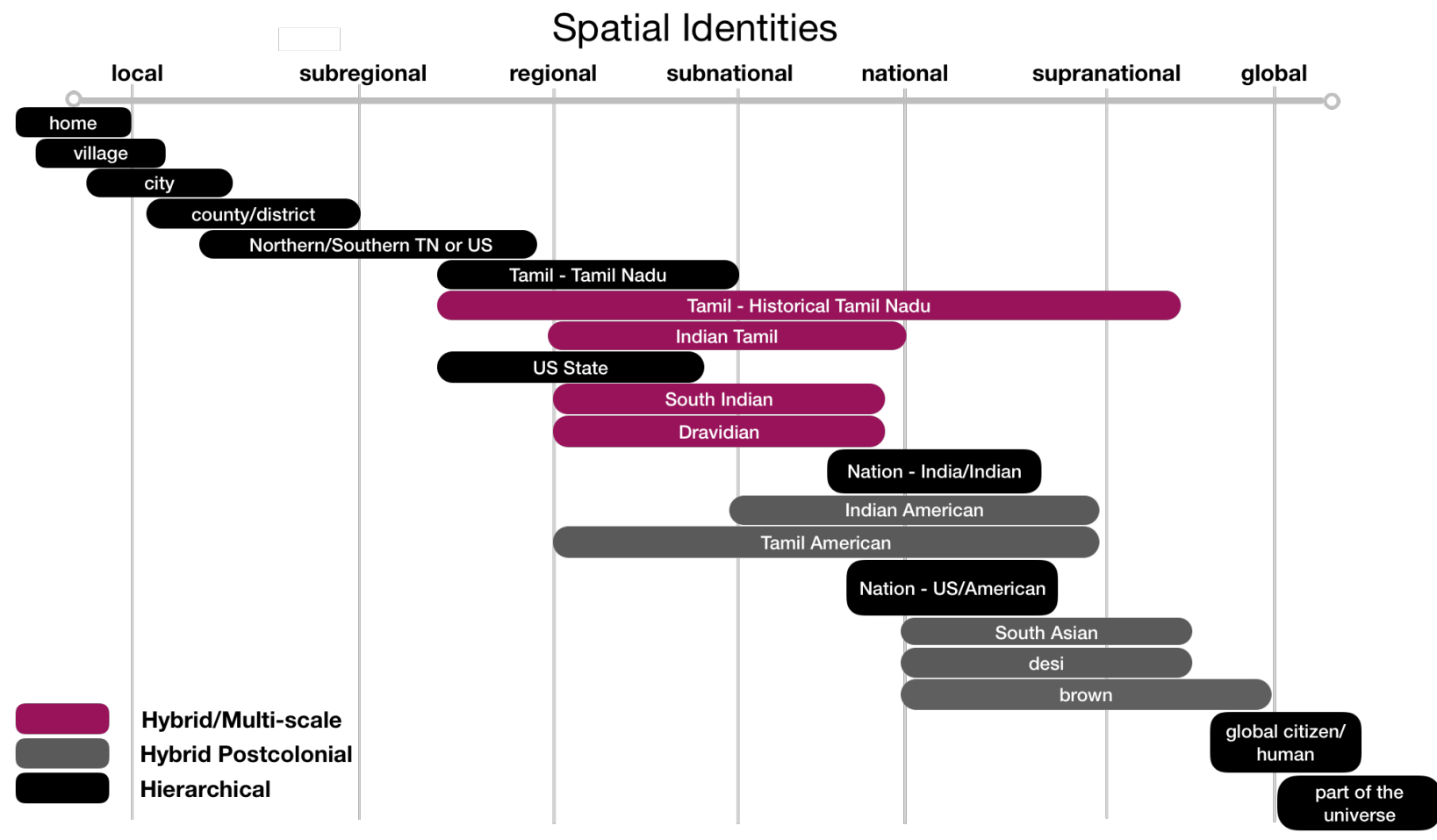

Figure 3: Spatially-oriented identities referenced by participants, demonstrating relationships between scale and hybridity.

Note* there is some overlap between hierarchical, hybrid/multiscale, and hybrid postcolonial. I graphed these however, with respect to the ways in which participants used them most frequently.

\section{Hybridity and Scale Debates}

While debates regarding scale flourish in geography, those that focus on hybridity and scale are limited. Bhabha (2015) is one of the few scholars to link hybridity with scale. Some scholars have used the term hybrid but have used it as a descriptor rather than connecting it to the history of colonialism (Bhabha, 2015). Bhabha (1994) suggests that hybrid identities often rely on spaces of in-between and thus inhabit in-between scales or even multiple scales. He discusses the links between scale and hybridity through connecting to in-between and contradictory spaces. "Indeed, it is in relation to these 'ordering' principles that hybridity derives its agency by activating liminal and ambivalent 
positions in-between forms of identification that may be asymmetrical, disjunctive and contradictory" (Bhabha, 2015, p. xi).

Bhabha (2015) ties hybridity to scale through diversity - where diversity itself becomes a scale. "The claims of global hybridity rest increasingly on the scale of diversity, not on the diversity of scale" (Bhabha, 2015, p. ix). Although connectivity is more prominent in a global world, scalar diversity is still important. Essentially, he argues that academic use of hybridity has been ambiguous and imprecise, and thus only contributes to homogenizing hybridity through global scale. I've already discussed the importance of dehomogenizing diaspora, but purposeful dehomogenization is also important for many communities within larger diasporas, especially regional identities that actively distinguish themselves from national identities (Johnson and Coleman, 2012). So, while some scalar identities, like Tamil, are listed as hierarchical (see Figure 3), participants at times, used this identity to purposefully challenge and dehomogenize identities, such as Indian, that emerged from a postcolonial context. Bhabha (2015) stresses the importance of using spatial, hierarchical scale like this with hybridity and more specifically local, global, national, and regional scales. Simply put, he suggests that the academic use of hybridity has emphasized global scale so much so that ignores context of locality, site, and specificity, important to nuanced and decolonized identities. Bhabha thinks of scale in very hierarchical categories, much like those who identify with flat ontologies would reject.

Yes, hybridity has been recruited into the service of global homogeneity. The largescale global frame pluralizes the conflicting rights and interests of demographic, democratic and global differences. The specifics of site, locality, history and territory 
are seen as nothing more that the moving parts of a larger pattern that only becomes meaningful when it is subsumed into a synchronic global design (Bhabha, 2015, p. ix).

In other words, identities are discussed simply through a global homogenized identity. This is problematic to Bhabha (2015) and Papastergiadis (2015), who suggest that the way that hybridity is implemented in current social science serves as a tool of erasure that removes any semblance of origin or history. Bhabha's (2015) critiques hybridity for no longer including nuance, suggesting scale is important to contextualizing hybridity.

Scale and hybridity can be used simultaneously to conceptualize complex, diasporic identity. They offer different yet connected insights into how people view and understand their identities. Hybridity informs how identities are blurred, hyphenated, or connected to colonialism. For example, through interviews I found that some participants viewed themselves as Indian-American or Indian-Tamil and saw these identities as interconnected and inseparable. Yet, others saw that these identities were quite binary and not representative of their "in-between" identities because they still mimicked colonial representations of authenticity or purity. In other words, colonial binaries of purity persisted in how people thought about their identities. For many, identities like American were associated with whiteness through qualities or characteristics, and by virtue of being brown, Indians or Tamils could not be fully American. I discuss this more in Chapters 6 and 7, connecting it to Critical Race Theory. Hybridity demonstrates connections to the colonial past and seemingly irreconcilable binaries constructed by colonial imaginings often linked to whiteness and purity; yet also demonstrates that these identities are fluid and in flux. 
Scale identifies when participants solidify identities with specific local, global, national, or even supranational identities, but is also connected to colonialism in that it can be used for the purpose of dismantling rigid binaries, products of colonialism.

Scale has multiple definitions as discussed in Chapter 2, but this research demonstrates the importance of hierarchical scale, that can also at times be hybrid, and multi-scaled. In this way, scale tells us how "human interactions" as Marston (2000) suggests, "create those scales." Scale, in the context of interviews, also remains hierarchical with categories like national, regional, subregional, etc., for many participants when conceptualizing identity. Yet, at times, this hierarchical scale shifted to multi and hybrid scales such as Indian-American, Indian-Tamil, Indian Tamil American, etc. In other words, participants' experiences and how they view representations of identities, inform how they use specific scales to define identity - either small-scale, as Raj did with a specific village with other Tamils or on a larger-scale, as Indian, in the context of the US.

\section{Scale and Hybridity existing simultaneously}

Scale and hybridity are much broader concepts, not always connected to identity. But, when connected to identity, scholars have thought of traditional scales and hybridity as contradictory for three reasons. First, in general, scale with regard to identity gives importance to specific origins, while hybridity at times can imply that the idea of origin is multiple or non-existent. As Bhabha (2015) voices, current use of hybridity is subsumed by "global homogeneity" that erases "origin." In this way, the concepts seem irreconcilable because scale implies an origin, while the use of hybridity that Bhabha critiques implies no origin. But, as Bhabha (2015) notes, hybridity does not need to be applied to suggest no origins, which is why he begins to draw on scale as it informs a deeper connection to origin 
or place. Second, the use of scale as Marston (2000) describes implies that scale is evoked, practiced or performed, while hybridity "is born out of the transgression of this boundary, figures as a form of danger, loss, and degeneration" (Papastergiadis, 2015, p. 259). Scale highlights boundaries, while hybridity blurs them. In relation to identity, however, identity is always in flux, unstable, and changes based on moment. Therefore, for the purposes of identity, boundaries may exist in one moment, yet lose importance or disappear in another moment. Third, hybridity has a specific historical context that links to colonial and imperial effects and does not fit into categorical boundaries of traditional, hierarchical scales. For example, pre-colonial notions of Tamil, often referenced by participants, do not conform to current boundaries or conceptualizations of post-colonial Tamil Nadu. Yet, people exist and inhabit these conceptions of identity daily.

But, if thinking about how scales and hybridity surface in moments, it is possible for hierarchical scale and hybridity to exist simultaneously. Hybridity does not have to only imply no origins but can at times can emphasize origins. Though it often implies not having singular origins and thus no fixed origins, it can also imply multiple origins, connecting it in many ways to hierarchical scale. As Papastergiadis (Papastergiadis, 2015, p. 259) states:

If however, the boundary is marked positively - to solicit exchange and inclusionthen the hybrid may yield strength and vitality. Hence the conventional value of the hybrid is always positioned in relation to the value of purity, along axes of inclusion and exclusion. In some circumstances, the 'curse' of hybridity is seen as a mixed blessing. 
When he refers to the "mixed blessing", he explains that hybridity can also work as a double inclusion from many sources - that the hybrid is not just exclusion, but also inclusion. It can be a way to bridge identities and groups of people that does not just require cultural purity, but instead embraces cultural multiplicity. At the same time, hybridity in other circumstances can still imply loss and coexist with scale. For example, some participants at times described their identities as very local, with villages or towns, but in other moments identified with broader identities like Indian or South Asian. Overall, their identities blurred and shifted, but solidified through scale in particular moments.

In other words, people can have hybrid identities, yet also describe them with hierarchical scales, like regional such as Tamil, perhaps even more broad like South Indian, national like Indian, or almost supranational in reference to South Asian or desi, in specific moments of time to mobilize specific identities and dismantle colonial binaries. They can even mobilize multiple scales to embody these identities. This does not have to fit under traditional hierarchical categories such as national, diasporic, or regional - rather it can be theorized as multiple scales or even hybrid scales at times. For example, some participants who described themselves as Indian, described themselves as Indian through a regional lens like Tamil, subsequently characterizing their identity as Indian-Tamil. Some even used framed Indian through more local scales like villages or districts that blurred with Tamil and Indian. Indian was a national scale, but also multi-scalar and hybrid, blurring all of these scales at times, and solidifying them as separate in others.

Scale much like hybridity, as Nicely (2009) indicates, is multiple, contradictory, and for specific case studies can constitute "alternative narratives and subsumed geopolitical actors at various scales and places" (Nicely, 2009, p. 20). Nicely (2009) stresses that 
"consideration of geopolitical narratives would benefit from a complementary focus upon these situated, meaning-laden practices of place-bound individuals heterogeneously constituted through racial, ethnic, class, gender, and other identities" (Nicely, 2009, p. 20). Nicely (2009) theorizes intersectional scale that she later characterizes as hybrid, pointing out that two different scales can exist simultaneously.

Nicely (2009) critiques Gerard Toal's (2004) essay on the 2004 terrorist and counter-terrorist attacks in Beslan, North Ossetia. She explains that trying to separate the scale or place them in hierarchical order is not always helpful and becomes more of an academic exercise than a reflection of everyday life. She claims that the North Ossetian understandings of the attacks are shaped by many geopolitical actors including the Kremlin, North Ossetia, the terrorists, and many others. These scales exist simultaneously and inform one another. But, as Johnson and Coleman (2012) argue, scales can exist simultaneously and inform one another, but yet can also be mobilized during specific moments for specific purposes. Essentially, as they argue, regional scalar identities can be purposefully mobilized to counteract dominant, national discourses, even if both national and regional scalar identities exist simultaneously and inform one another.

Additionally, while Nicely (2009) calls for greater emphasis on hybrid and multiscalar research, she does not place it in the context of diasporic hybridity, which requires a postcolonial context. In my own work, hybridity and hybrid scales are different concepts and while they overlap, they are separate. Hybrid scales implies that scales can be multiple, shift, and exist in spaces of "in-between" (Nicely, 2009); but, hybridity is a condition that describes spaces of "in-between" created directly by the legacy of colonialism (Bhabha, 1994). In other words, hybrid scales signify that scales are in flux and changing, but 
hybridity is an underlying process of post-colonial identity informed by specific histories and context.

I use many definitions of scale - hierarchical, multiple, and hybrid scales, but I see these as separate concepts from hybridity, yet also stress that work together. Scales can be multiple and even hybrid, but hybridity as a concept implies something much deeper, informed by historical processes of colonialism. Hybridity shows how identities come from multiple sources and compete but does not directly identify purposeful mobilization of specific scalar identities as counter-narratives to dominant nation-states or national scales (Bhatia, 2007). As Hartman (2008) or Gilroy (1993) suggest, these identities are transitional, and do not have singular origins. Yet, people can still draw on these types of origins through scales like national or regional through India, Tamil Nadu, or America to inhabit and experience their identities within specific moments. Someone can be Indian, but perhaps is only Indian under specific contexts. Nevertheless, they inhabit the idea of "Indian" in specific moments, evoking the use of a "national" identity. At other times, they can inhabit a "regional" identity, but at the same time also be American, Indian, or even transnational without specific ties to a particular origin. In other words, concepts like hybridity and scale are fluid and contribute to a larger synthesis of a complex, situational, represented, and embodied identity.

Identity can be both scaled and hybrid. In the next few sections, I provide examples of participants who express both hybrid and scaled identities when navigating identity in the United States and examples of how these concepts informed events and performances. In this analysis, I use scale and hybridity not as either/or but rather as two separate but linked concepts. Scale, for participants, though it is socially constructed, still manifests as 
traditional notion of hierarchical scales, but changes with moment and situation. I am not suggesting that these scales are not socially-constructed, but rather that participants view them as categorical, much like traditional notions of scale. In other words, participants perform these scales in everyday life. These scales are not always fixed, but can be blurred, fluid, and effected by hybridity.

\section{Varying Hybrid and Scalar Identities}

In each interview, after explaining the definition of identity in the social sciences, I asked participants to describe their identities. Participants described multiple identities, often intersecting with religion, gender, sexuality, race, among others. The three most prominent descriptions of identity included Tamil, Indian (sometimes South Indian), and finally American. These were conceptualized in very scalar ways and depended on situation and context. They were scaled hierarchically in moments when participants would describe Tamil as opposed to broader Indian or Indian as opposed to American in the broader US context. These were also hybrid. Although participants conceptualized these identities as quite hierarchical at times, they also described experiences where these identities were hybrid, blurred, or in-between.

Within each of these identities was nuance, especially scalar nuance related to state, city, town, village, or region. Many participants identified with a specific region, village, or area of Tamil Nadu or India or as a larger, more broad, all-encompassing identity like South Asian. In many cases, participants did not feel comfortable identifying as Indian, but would identify as South Indian. Sometimes participants would say "global citizen", indicating that they did not identify with any particular area and thought of the idea of a unified world, but 
would also mention "Tamil" as that shaped their experience. Much like Nicely (2009) described, this was an example of co-existing, multiple scales which happened frequently. This happens with many groups and people, but what was particularly noteworthy in the Indian Tamil diaspora was that there were multiple layers of scale, from village to broader South Asian and desi that were informed by discrimination on multiple scalar levels and had embedded effects of colonialism through hybridity and mimicry. This made definitions of Indian Tamil identity quite varied and complex.

The level of scalar identification, though, varied from person to person. I also found that participants described identities as hybrid or connected, like Indian-American. These were not always necessarily described as hybrid scales, however. Some participants linked these identities to a condition of hybridity informed by post-colonial processes. They described hybrid identities would often say that they do not really separate those identities or that they identified as such because they did not fit into one or the other. For example, one participant mentioned that she has to identify as Indian-American together because she feels neither fully Indian, nor fully American. While in one sense, this is a hybrid scale, it is also a product of the postcolonial dispersion of formerly-colonized diaspora that more directly falls under the concept of post-colonial hybridity. She was not just using scale to describe her identity but relied on the concept of a hyphenated identity, informed by spaces of in-between. Her identity was scaled, but also hybrid.

She was Indian in certain situations, American in others, but overall IndianAmerican, because each of her experiences were shaped by the other. In this case, she described both scaled and hybrid identities, much like Nicely (2009) and Bhabha (1994) indicate. She did not fit fully into either precisely because as Bhabha (1994) suggests of 
hybridity, the colonial legacy of binaries has been reinforced in diasporas. As Fanon (1967) and Bhabha (1994) both suggest, certain identities in postcolonial contexts are also linked to whiteness. Especially in a post-Trump election era, many participants described that they did not know if they could be fully American because American was now being pushed as culturally white. Yet she was also both, existing in-between and inhabiting both identities as Papastergiadis (2015) suggests is also true of post-colonial hybridity. She expressed scaled identity in moments as Marston (2000) suggests, but yet, also expressed a hybrid, blurred identity.

Both hybridity and scale intersected with many participants' identities. Participants described identities through the condition of hybridity and simultaneously, discussed scalar nuances of each of these identities. They described themselves as having multiple identities - they were Indian and Tamil or Indian, American, and Tamil, or sometimes, they were Indian and American and only Tamil because their families came from that region of India. Specific language used by participants included Indian-American, American-Indian, Indian-Tamil, Tamil-Indian, Tamil-American, or Tamil with a mention of a specific part of Tamil Nadu. Singular identity was rarely described and was often hyphenated and inbetween multiple identities. This was true for all participants, regardless of generation or other factors, which I will discuss further in Chapter 7.

Many participants described tensions and struggles of blurred in-between identities. These identities were hybrid, but also scalar in moments. For some, small-scale identification with villages or towns was crucial, while for others, large-scale identification with national or supranational identities was much more important, depending on situation and context. In other moments, they hybridized Indian as Indian-American or 
Tamil-Indian or Indian-Tamil. Others chose to describe themselves as South Indian, and in some cases, scaling broader to discuss their identity as South Asian. Some chose to use the word "desi" instead of Indian, also using a broader scale to describe their identity. Many South Asians use the term desi to describe people of South Asian descent. Desi served as a more all-encompassing term that indicated South Asian. In a few cases, participants used South Asian, desi, Indian, South Indian to describe their identity as Indian. These participants blurred the identities of South Asian, desi, and Indian but simultaneously described that their identities were informed by a particular scalar lens of South Indian or Tamil.

\section{Complex Identities}

The way that people described their identities varied significantly but hierarchical scales reflected in specific terms like Indian, South Indian, desi, South Asian, Tamil, etc. surfaced consistently. These hierarchical scales were not always reflected in a top-down way, but instead developed "at the moment" (Ferber \& Harris, 2011; Marston, 2000). For Nazeem, who identifies as Muslim, American, desi, woman, and also Tamil “by convenience" as she describes, she was much more comfortable describing her identity broadly at the supranational scale, often referring to herself as desi. Nazeem's identities were also beyond scalar as she addressed multiple intersections and categories including gender, religion, region, and traversed many boundaries. She is not able to identify with a specific regional lens of Tamil like some, or even with the hybrid/blurred category IndianTamil. She identifies more supranationally as "South Asian" or "desi" because her parents are from India, but lived in Pakistan making her Pakistani, Indian, and Tamil - evoking multiple national and regional scales. Unlike Nazeem, Raj, whose interview opened this 
chapter, identified with a specific village. This changed for him, though, based on situation and context as he had to scale up to identify at times as Indian with others who were not familiar with "the geography of India."

While Raj broadened his identity, he still feels strongly connected to his local village, even in the US. Though, he says, his identity shifts over the years as he lives and works in new places and is surrounded by new people. At times, he has to identify nationally as Indian, because that is how others see him. Maari, similar to Raj, also identifies with his small town near Tiruchirappalli where, he says, "everybody knows everybody" and everyone is like "family." Maari does identify with a national scale of Indian, but rather sees it as a country where he is a citizen. He says he is strongly Tamilian, identifying with the state of Tamil Nadu, and then describes his identity more locally as a Tamilian from Trichy. Now that he has been in the US for a few years, he says he identifies as American only because he has lived here and has friends here. "In my heart, I'll always be a guy from Trichy."

Although all three are positioned between multiple identities and hierarchical scales, but Nazeem does not pinpoint her identity to such a specific scale. She often uses a broad, large scale, supranational scale to describe her identity. But identity is not just scalar for Nazeem and many others. Nazeem describes identity as hybrid and shifting, noting that she weaves in and out of different identities. For someone like Nazeem, her scalar identities were affected by the changing borders and boundaries set in place and reinforced by colonial powers. Before partition and colonial rule, Pakistan and India were not divided by borders and Tamil Nadu was not even connected to North India (Edney, 1997). The way that she thinks about these identities currently are in a postcolonial context, but her 
family's history spans the pre-colonial context. Thus, these identities have become hybrid and in-between multiple rigid, often binaries like Indian/Pakistani.

For Nazeem, she sometimes describes these identities hybrid precisely because they are so complex and have a very specific history. However, other times, she describes them as scalar, often hierarchical, depending on situation and context. She uses her mom as an example. "My mom is a super Tamil type with her Tamil friends, again, that is a language thing, but like, she is not going to capitalize on being South Indian with her Pakistani friends." She shifts from talking about identity at the scale of the nation - Pakistani, to a smaller, regional scale of Tamil, and then broader to a subregional scale of South Indian. Though, Nazeem says she does not speak Tamil so feels that she is much less Tamil than her parents. Yet, she identifies with being Tamil, Pakistani, Indian, desi, or South Asian based on company and situation.

She compares this to her mom's experience. She describes it as both a conscious shift, but also a very emotional and sometimes involuntary shift. It becomes relational, common with many identities.

I feel very American when I'm amongst "real" Pakistanis or desis. When I'm around international students and expats I'm like 'I'm so American.' Then I'm around American friends and I'm like 'god, I'm so not American.' Largely, I feel that, when I look at my parents and my values, I definitely see myself more as American relative to them, but then you know, especially since 9/11 - what does being American even mean and is there room to be both? Or to identify as desi and Muslim and American - because people kind of expect you to choose one. I do consider myself American - 
but I feel that means something very different to different people. I was born here, raised here, and couldn't imagine myself to live anywhere else. I don't have a desire to go back to the motherland and live there. To visit there is ok, but that isn't my home. I cannot relate to someone who was raised there, there is too much of here in me for that to work (referring to arranged marriage).

But, while her identity is relational, it is also hybrid. For Nazeem, these identities are not clear-cut, and she does not fit into the represented binaries of identities created by colonialism, thus creating a space of hybridity or in-between. In other words, there is no pure definition of Indian, South Asian, or other identities. Therefore, she often finds herself in-between. She straddles multiple identities that change based on situation, context, and even political climate. Some identities, she feels, are positioned in a way where they cannot coexist, like being Muslim and American in current political climates. As Bhabha (1994) suggests, these identities are pitted against one another because they do not fit into spaces of authenticity. These related to hybridity because they are neither 100 percent the colonizer nor 100 percent the" Other," and therefore create a sense of hybrid that is not represented in the space which Nazeem lives. Hybridity is complex and is not simply the blending of identities. As Chacko and Menon (2013, p. 99) indicate, “... hybridity is not simply the fusion of two binarized categories of identity; hybridity instead destabilizes the fixity of these categories". Hybridity demonstrates that these categories, identities, and even scales are in flux and can overlap.

Nazeem's identities are also informed by implications of political context, described in the Introduction. She said that before $9 / 11$, she might have identified first at the 
supranational scale of desi, but after $9 / 11$ found that she identified first with a more hybrid, blurred identity - being Muslim AND American - Muslim-American. She says this became a purposeful statement about this identity in very Islamophobic climates. She purposefully hybridized her identity. After the recent election, she says she is now even more aware that she is a woman. Gender is not the focus of my study, but I want to demonstrate its importance to political context of identity. She says being a woman in a post-Trump election US is scary as an American. Political climate is significant for how Nazeem views, scales, and even hybridizes all of her identities, including her religious and gendered identity. In turn, her religious and gendered identities do not just influence how she sees being American, but also influence the way she views being desi:

When I think of Pakistani or desi standards for women, it's like oh, what you're expected to be a good wife, when you go to father's home to your husband's home, it is not even that you marry at 20 years old any more, used to marry earlier, it went from that to now - ok you are here now, you are going to go to school and get a good degree and you are still going to be Susie homemaker and then stay home and have kids and he is going to make enough money for both of us.

She says that being American gives her options and standards to choose how she wants to live - or at least, she thought it did. She says that it still does but changes often with political climate. Being American is also significantly problematic for Nazeem with the rise of Islamophobic and sexist rhetoric. Nazeem is one of many participants who described situation, place, and political climate as affecting their views of their identities. She is also 
one of many who described the issues of having a hybrid identity and never fitting into spaces of authenticity; yet also conceptualized her identity through traditional, hierarchical scales. More importantly, she is one of many who purposefully hybridized or identified with specific scales, depending on context and situation.

Santhya, for example, who now lives in the US, grew up in Switzerland and identified at the national scale of Indian. She thinks of herself as part of "Western culture," but said that she always felt Indian until she lived in India for a few years.

I felt very out of place there. I met my husband's friends' wives and they were all materialistic. I've never been friends with people like that. In India, I didn't have the confidence to make my own friends. I left feeling disappointed that I wasn't as Indian as I thought. But I also left with a peace knowing that ok, I look this way, I have this name, knowledge of these languages, but I'm much more comfortable living in a Western society.

Her identity was somewhere in-between. She was not Indian, but not fully "Western" either. Even living in the US, she still struggles with the hybridity of being Indian and Tamil. "If you are a generation that is growing up in the West and you don't speak your language (referring to Tamil) - you are a failure. I still feel inadequate that I don't speak it properly. There is a feeling that this is my language and I can't speak it properly." She said that this feeling of inadequacy is beyond just speaking a language, but a part of struggling with hybrid identities. Colonial binaries that reinforce whiteness pervade even in these situations. In other words, her brownness - a concept elaborated on further in Chapter 6 
and 7, requires her to fit into a pure "Indian" or "Tamil" identity. For many participants, "society" or power structures in the world, forced them to think about identities in a very non-fluid, non-hybrid way, when they found that these identities were in fact quite blurred and hybrid. As Nazeem described, it forces people to "choose" identities, when in reality, identities change and shift continuously.

In the same way, traditional, hierarchical scale was also not stable. While participants used specific scales in some moments, they were forced to use broader scales in others. Participants like Maari or Raj described that while they hold onto their village identities strongly and feel very much at home when they visit, they are still forced to identify as Indian with groups of people, often Americans, who do not recognize the nuances of places like India. In other words, they conceptualize their identities within hierarchical scales, but also demonstrate significant hybridity and fluidity in their identities.

\section{Tensions between identities}

While participants described hybrid or scalar identities, they did not describe the same identities nor agreed what those identities signified. In other words, "Indian," even when used as a national scale, did not mean the same thing to all participants. The same was true of "Tamil" and "American," indicating that "Indian," “Tamil," or even "American" were contested, heterogeneous identities. That is not to suggest that these are not valid categories or tools of inquiry, but rather to demonstrate that while people identify strongly with these identities, they often define them quite differently and sometimes in opposition 
to or overlapping with one another. As Bhabha (1994), Kraidy (2005) and Kaplan (2018) have described, identities can be contradictory, yet exist simultaneously.

In general, academics acknowledge that identities are not singular, are contradictory, and also fluid. But outside of academia, and what many of my participants expressed, is that in certain moments they feel their identities have to be rigid and fixed, and often used them in very traditional, hierarchical ways. In these moments, not only are identities often treated as homogenous, but certain identities are seen as threats or serve to disrupt other identities. For example, the idea of "American" is represented through societal rhetoric suggests "we are all American." Yet, academics acknowledge the complexity of such American identity suggesting that it is an imagined concept (Jansson, 2010).

There was not one way to define Tamil, Indian, or even American. American, for Nazeem, was a sense of freedom, but it was also a burden or threat because of the rise in Islamophobia, described earlier in this chapter. For other participants, American was the identity of opportunity and many participants associated it with capitalism and the "free market." Yet, to others, American was about giving and charity or alternatively, a threat of Western values waiting to dismantle traditional family values of "Indian" or "Tamil" culture.

Indian, like American, varied from being a set of cultural practices to a place where someone's family was from or even existing as a threat to Tamil identity. Indian identity became a scalar war and for many, it was the erasure of regional identities like Tamil. Devadas and Velayutham (2008) argue that cultural dominance, for example, through the influence of Bollywood, creates a hegemonic nationalism and national identity of India, 
largely shaped around North Indian narratives, thus marginalizing counter-narratives and histories of the South. For some, Indian was a large-scale that erased smaller-scale identities like Tamil or city and village-based identities. Even Tamil identity was sometimes described as a regional, small-scale threat to destabilizing unity of Indian, much like Johnson and Coleman (2012) describe of the process of creating national identities through opposition of regional identities. Regional identities often work to oppose dominant, national narratives, thus creating a regional opposition or redefining of national culture (Johnson and Coleman 2012). Regional, scalar identities outside of dominant national narratives can be painted as "backward" or inhibitive of national progress by everyday populations or in films and other mass media representations (Jacob, 2009; Johnson \& Coleman, 2012).

On the other hand, Tamil was also described as too large-scale and a threat silencing small-scale village identities or non-dominant rural identities. For some, Tamil identity could be used to reinforce dominant Brahmin, upper-class, or Hindu narratives of Tamil communities. Not only were each of these identities defined differently, but they were often defined against one another. Yet, these identities could also be hybrid in blurring lines between regional, village, and other identities. They exhibited hybridity in that they employed postcolonial mimicry of colonial mindsets, discussed in Chapter 8. Some discussed their identities and both Indian and American. The identities were defined in opposition to one another but co-existed to create a very specific identity. They also described these identities as reasons that they did not fit into binary categories and thus, as Chacko and Menon (2013, p. 99) describe, "destabilize(d) the fixity of these categories" like Indian, American, or Tamil. Participants often found themselves straddling multiple 
identities and rarely described identities without overlap at some point. Simply put, identities were discussed as hierarchical and separate at times, reinforced in specific moments as Marston (2000) stresses. Yet, they were also fluid, complimentary, co-existing simultaneously, blurred, and hybrid as Nicely (2009) describes.

\section{Contradictory, opposing, and fluid scalar and hybrid identities}

Participants also described identities, like Indian and Tamil, often described in a hierarchical scalar way, as being opposed to one another or describing tensions between them. These identities were dependent one another and co-constructed as Johnson and Coleman (2012) suggest. Some participants prefaced national 'Indian' as more important than regional 'Tamil' for specific political reasons. People who identified too strongly as regionally 'Tamil,' threatened to destabilize the broader national identity of 'Indian.' This was both a problem of scale and hybridity. It was a problem of hybridity because those who saw themselves as both nationally Indian and regionally Tamil, sometimes found these connected and blurred. These identities were seen as opposed by some, yet for others these identities were inseparable and could exist without the other. Colonial representations that simplified India into a single history and emphasized purity or authenticity sometimes overpowered complex and rich histories. For example, in my interview with Arvind, he said described that Tamil history was rich and often overpowered by dominant North Indian narratives that took precedent at the creation of India. Tensions between identities were connected to first, a history before colonialism, i.e. the often-referenced ancient history of Tamil Nadu, and second, the product of unified India post-colonialism, i.e. post-1947 India. 
This was visible not only in participant interviews, but also in much of my participant observational research that I discuss later in this section.

While hybridity was indeed important to understanding tensions between identities like Indian and Tamil, this was also a problem of scale, because as some participants described and some events referenced, the scale of Tamil Nadu's history was often lost within broader national Indian history that focused on subnational North Indian narratives. Yet, this scale developed "at the moment" (Marston 2000; Ferber and Harris, 2013, 190), giving meaning to particular regional and national scales in specific contexts. Participants drew multiple meanings from each of these 'scales.'

\section{Hybrid and Scalar Indian vs Tamil}

Vijaya, who came from Tamil Nadu to the US five years ago, says it is important for her and even others to identify more broadly as Indian because otherwise, she would fall into the "divisive" groups of Tamils. That is, those who identify regionally as Tamil first before nationally as Indian. “I don’t like to belong to a particular group. I want to be a generalized Indian. I like to speak to any people, not just to Tamil people. I can mix along with all the other people well. I can up mix up with any people and talk to them freely." She says this is part of being Indian. "Indian means to be - the family cultural values, I just respect that the most. That is from Indian origin. I like the joint family culture. I like to be as a joined family."

Diya, who came to the US around 10 years ago, also describes herself as nationally Indian first. She says that it is an important distinction to make. "I am an Indian first, and then I am Tamilian. I was saying I was Tamilian and I'm so proud of it. But I'm from India. I 
don't think I can weigh that on that scale, because in my state, there are many people who consider themselves as Tamilian first and then only Indian. The people who love literature, they consider more Tamilian and then Indian." She said this can be problematic because it creates divisions for Indians, although, she says there is nothing wrong with loving all Tamil things. She says that India is a very diverse place, but sometimes there is too much diversity. "In India I think we have too much. I'm not that kind of person. India is so diverse; every state has their own events. And the other states have no idea. We have like Diwali and others. But if you go to Andhra ${ }^{13}$ and all they have different events." Yet, she says, many Indian communities try to make universal events for everyone to participate. She sees this as positive. In her interview though, she often made distinctions between Tamils and other Indians, in essence, reifying the divisions that she wanted to minimize. Many participants who wanted to minimize difference, did reify these differences throughout interviews, which I describe throughout the next few chapters. Most participants agreed that "diversity is good", but for those like Diya, Vijaya, or others, diversity can mean division. That is to say that the idea of being nationally Indian is often at odds with being a person from a regional state of Tamil Nadu. During a visit to New Jersey, I interviewed two participants separately. These participants knew one another, and I spent the day with both of them. Later, after the interview while we gathered for dinner, they discussed the questions of my interview. Lakshmi and Arvind both who came to the US in the 70s from Tamil Nadu, discussed the questions on how they identified postinterview. Lakshmi was adamant that it was important to identify nationally as Indian first

\footnotetext{
${ }^{13}$ Andhara Pradesh is a state north of Tamil Nadu
} 
and then with Tamil second. Arvind said, "absolutely not, you are giving up your heritage of being Tamil to identify as Indian." They discussed back and forth for almost 30 minutes.

Arvind said in his interview and repeated to Lakshmi that even his daughter had changed how he viewed being Indian when she wrote a piece on why they had nothing in common with other Indians other than coming from the same country. He said to me:

Since we have lived in 50 years, my daughter answered once, I am not an Indian my daughter only identified as a Tamilian as opposed to an Indian. Or even more regional settings (referring to specific parts, cities and towns of Tamil Nadu). I have not one thing in common with other Indians. It is group behavior. Suppose I represent an Indian association, we have common issues we want to talk about. When I talk about Indians, I cannot talk for everybody.

He said that each Indian group has different languages, cultures, traditions, views, and all of these things make it difficult to identify as Indian.

Lakshmi said she felt very differently about this issue. This was in part, she said, because her father was a freedom fighter in Tamil Nadu during India's independence. For her, his fight against British colonization only solidified the idea that national identity of Indian needs to be before regional or subnational identities. Her father devoted his life to achieve unity of a national India and thwart colonial oppression. Yet, even after India achieved independence, she said he felt the country was divided. She said that her father was attacked during protests (in Tamil Nadu) because he would not say that "compulsory Hindi was bad." She brought this to current Indian diaspora politics, stressing that 
Tamilians often get upset when Hindi-speaking people assume they speak Hindi or only speak in Hindi and will say 'they are being rude North Indians.' But, she said, "it is on me for not learning my national language. We are all speaking in English, but that is not our country's language." English is the language of the British and those who colonized India. She says unity is important and the nation comes first.

Arvind said he understands where she is coming from, but that this ignores the national domination of the Hindi language. He also ties this into colonialism. He asks why Hindi is supposed to be superior to Tamil, or why Hindi needs to be the national language, and also why they are expected to change the way they have lived for thousands of years to fit in with people they have nothing in common with. The only reason that North and South India were together was precisely because of British occupation. While they tried to understand where the other came from, neither changed their mind.

Lakshmi, at a separate time, again brought this issue up to another participant who also sided with identifying regionally as Tamil-first ${ }^{14}$ and a similar conversation ensued. Lakshmi was not the only participant to feel this way, however. It seemed to be a struggle for quite a few and many prefaced their insights by saying that many Tamils do not agree with them. Identifying too strongly and regionally as Tamil threatened national Indian unity, while identifying too nationally as Indian threatened the very existence of regional identities like Tamil. In this instance and in many other interviews, both identities were pitted against one another as if they could not co-exist. Again, this problem was scalar in that participants voiced using one scale over another..$^{15}$ Socially-constructed and historical

\footnotetext{
14 This was a term used by participants.

15 No patterns emerged with factors such as where participants lived, gender, age, location, etc. In fact, members in the same family often had opposing views on this subject.
} 
meaning was given to regional and national scales. Each of these scales was then used to highlight a dominant culture or identity in the case of 'Indian' and as a means to resist dominant identities, i.e. 'Tamil.' Hybridity was also important in that the historical context of colonialism and colonial oppression bled into the very definition of these identities, in both first and second generations. Each participant described these identities initially as hierarchical and sometimes opposed, but at other times, they informed one another and were very much blurred and hybrid. They were also hybrid in that they were informed by tensions created through a legacy of colonialism. The importance of colonial resistance and co-creation of India by Indians was set in opposition to the "thousands of years" of Tamil history erased by the threat of India as the remaining product of colonial oppression. Participants could not reconcile differences because they described that choosing one rendered the other "inauthentic," thus reinforcing colonial binaries.

The differences between Tamil and Indian were also influenced by specific life experiences. For example, Lakshmi's father's experience was very important to her, while Arvind's daughter's experience was important to him. Lakshmi participated in many North Indian functions with friends while Arvind stayed mainly in Tamil and South Indian crowds. Though, for many participants who did attend North Indian functions and had many North Indian friends, this reinforced the differences between being Tamil and Indian. Life experiences were not indicative of how individuals would view Tamil and Indian as sources of division, but rather how they viewed or reacted to particular situations and experiences. It was an identity forced upon some, the last remnants of colonialism, while it was embraced by others, as a symbol of colonial defeat. Tamil was a threat to Indian 
national identity to some, while Indian was a threat to regional identity for others. Indian for some was a way of life, while for others, it was a national identity that they had no control over, while their regional identity was a means of resistance.

Rittika, who strongly identifies as Tamil, came to the US in 2013. When I asked about why she did not identify as Indian, she said, "I'm not, why? Because once I moved away from India only then I was identified as Indian. By immigration and others. There are no other circumstances in my life I was identified as Indian, because I was there so I didn't have to identify... We are not identified as Indians, though sometimes applications say Asian." For Rittika, Indian is a national identity that was given to her after she left home. Now that she is in the US, she says she is forced to scale up to Indian because that is what documents require. Many people in the US are not aware of India's diversity and so she ends up homogenizing her identity at the national scale. Only when she goes to Tamil functions and events and is around Tamil people is she able to express her Tamil identity and the regional and local differences embedded in her identities.

At events, I found that these scales were enacted in various ways. Much of the Indian community in Northeast Ohio and New Jersey identifies based on regional distinctions. Many participants that I interviewed often attended Tamil events rather than larger Indian events. In many of these events, pre-colonial Tamil history was often highlighted. Some said that they only felt Indian during national holidays. In fact, many saw the nationality of being Indian as just secondary to being Tamil. It was not necessarily in opposition to being Tamil. It was hybrid in the sense that it was connected and a space of in-between, but not laden with the entire colonial context in personal experience. Certain scales, like regional, were more important than national scales, like Indian. But, this was of course dependent on 
moment as Marston (2000) indicates. Paranthakan, who also came to the US in 2013 says that he generally feels more Tamil. He says it is important to be Tamil, but during national holidays, he also feels Indian. "I can describe more of my identity about Tamil rather than Indian. I do feel Indian during Independence Day, Republic Day, listening to Bollywood songs or watching cricket and so on. But mostly I feel as Tamil." Paranthankan would attend broader, larger scale Indian festivals that marked these holidays in Morgantown.

I attended some of these festivals with him. I found that Morgantown, had many broader, national-scale Indian events, and very few specific regional events. This was in part, according to many participants, because the Tamil community in Morgantown is much smaller. With smaller numbers, regional organizations and events are much less likely. Yet, even in these national 'Indian' events, attendees and organizers were often at odds with one another over what region of India to represent. Most of the events that I attended in Morgantown were broader "Indian" events. These were often tied to West Virginia University and organized by the Indian Students Association or AID (Association for India's Development) Morgantown. AID Morgantown connected Indians through giving back to India and raising money to support a cause focused on development. In fact, surveys were distributed asking Indians which region or area should be of concern for raising awareness and money. Answers varied as the community came from many different parts of India and sometimes did not know much about the area listed. It reminded me of my conversation with Arvind (and others) when he mentioned that with such a diverse group of people, “you can't represent everyone's interests." Aside from surveys, there were other discussions based on India's diversity. 
The Indian Students Association would organize events like TARANG around Diwali, which they have described as WVU's "principal event of diversity week" to bring together Indians from many parts of India. However, during my first observation, many attendees gave feedback saying that the event was catered mostly toward the Telugu community, who made up a large section of the participants. They mentioned that the songs, music, and audience were mostly in Telugu. The second and third year, the event incorporated skits, dances, film clips, and music from all over India. Some skits were designed to draw attention to the diversity and vastness of India's "rich cultural" background. Through music, dance, clothing, and film clips, they highlighted areas like Rajasthan, Gujarat, Punjab, Tamil Nadu, Andhara Pradesh and others. Throughout the latter programs, India's diversity was highlighted.

While Indian events are popular also in Cleveland and throughout New Jersey, many Indians also gather based on regional divisions. New Jersey boasts many regional societies and associations including one of the largest Tamil Sangams in the country, the New Jersey Tamil Sangam. Sangam derives from the Sangam Age of Tamil history (pre-colonial), where poets and scholars gathered under what is now referred to as a Sangam to promote learning, sharing, and education (Kalidos, 1976). "Sangam" is essentially a Tamil society or association, but the word connotes a direct connection to the purposeful learning of the Sangam Age. Present-day Sangams draw from ancient and pre-colonial Tamil history and literature for instruction, events, or performances. But, they also integrate and recognize American holidays like Thanksgiving and engage in activities like camping, fostering hybrid, blurred Tamil-American identities. In Ohio, events put on by the Tamil Sangam, the 
Bengali Cultural Society, the Kerala Association of Ohio, Odisha Society, among others as well as various language schools are quite popular.

In Cleveland, some of the regional societies are partners of the Federation of India Community Associations of Northeast Ohio (FICA), which began in 1962 as a student association. As of now, the Northeast Ohio Tamil Sangam (NEOTS) is not partnered specifically with this organization, but the Bengali Cultural Society, Marathi Mandal, and Odisha Society are listed on FICA's website as partner organizations. This organization seeks to:

Organize events that promote the rich cultural heritage of India in greater Cleveland; to facilitate ongoing dialog with civic leaders regarding issues of importance to Asian Indian families in the region; to facilitate the mainstreaming of Asian Indian families by creating a platform for the community's active participation in the civic life; to collaborate with local organizations to promote goodwill and understanding between diverse communities; to partner with business groups that promote ties between Indian and US firms and foster economic development.

FICA is proud to showcase their efforts to establish the India Garden as part of the Cleveland Cultural Gardens exhibit on Martin Luther King Jr. Blvd that promote diversity and inclusivity. On their website, they state that the seven-foot statue of Gandhi built by famous sculptor Gautam Pal of Kolkata is one of the largest statues of Gandhi in the United States. FICA describes that this statue serves as a unifying symbol of Indians in the Greater Cleveland area, emphasizing India at the national scale. FICA hosts events around Republic 
Day, (India) Independence Day, Deepavali, and Holi. Yet, many Indians choose to attend events through regional associations or through religious activity. Some participants I interviewed do not attend any national Indian events, nor do they belong to any associations. While these associations are part of the broader "Indian" community, they do not include all of the community. In fact, many Indian Tamils mentioned they preferred to either attend Tamil events, others attended events based on friends and family, and some, no events at all. Hamid, like others, for example, even made a point to purposefully not attend Indian or Tamil festivals. He told me, “I don't integrate with Indians outside of India. In America, I'm a world citizen. If someone asked me, I would say I'm Indian, but I don't need to prove to an Indian in America how Indian I am."

But most participants described that they had attended some Indian or Tamil festivals or events. Many who identified regionally Tamil before nationally as India, attended Tamil events. The Northeast Ohio Tamil Sangam, where I attended 15 events, describes itself as:

A membership based non-profit organization formed by the Tamil language speaking people of North East Ohio. NEOTS represents highly skilled professionals in various areas including Medical, Engineering and IT sectors hailing mainly from Tamil Nadu - India and Tamil Speaking expats from other parts of the world. The objectives of this organization are to promote the awareness of Tamil culture, Tamil heritage through social, cultural, literary, charitable and educational activities. 
Both the Northeast Ohio Tamil Sangam and New Jersey Tamil Sangam have organized fundraisers for aid to Tamil Nadu in the past for education or natural disasters but have also organized for aid to the US for disasters like Hurricane Harvey or even to provide clothing for "underprivileged" people our "local community" (this specifically referred to the Cleveland area). Beyond organizational efforts, in the Northeast Ohio Tamil Sangam functions, regionally Tamil identity was highlighted, followed by national identities of American, and finally Indian. Indian was strategically highlighted to define Tamil identity as being Indian. Yet, Tamil identity was also not the same as being Indian and Indian associated with Hindi or North India was minimized in most events, while Tamil was boasted. I elaborate further on this in Chapter 6 through examples of sound.

In essence, India was used to reify Tamil as Indian Tamil identity throughout events and performances. Tamil identity was associated often with Tamil festivals like Chithirai Thiruvizha (celebration of the Tamil New Year - Chithirai is the first month of the Tamil calendar), Pongal (Festival of the Harvest season), large Indian festivals like Deepavali (otherwise known as Diwali), Tamil food, language, and music. Tamil identity was represented in performances and events in different ways, depending on the year and audience. In some instances, music and dance performances featured Bharatanatyam and Karnatak music, often associated with very specific types of Tamil identity and not representative of all Tamils in Northeast Ohio. I further discuss these implications in Chapters 6 and 8. The Cleveland Thyagaraja Festival, not a part of the Northeast Ohio Tamil Sangam, but attended by some members who are part of NEOTS, celebrates a specific kind of music - Karnatak music, often available to upper class and caste Tamils. I will discuss these further in Chapter 6 and 8. 
Hierarchical scale and hybridity were both highlighted in a 2016 Pongal celebration put on by the Tamil Sangam. This celebration showcased a skit involving members of the community and their children. Some of this information introduces the importance of sound, which I will discuss in Chapter 6, but overall, demonstrates the ways in which these identities are navigated and represented. The opening of the program begins with Neerarum Kadaludutha, the invocation to the Goddess Tamil or Tamil mother, which is the state song of Tamil Nadu. In other events I attended, the United States Pledge of Allegiance follows or the National Anthem of the US. India's national anthem is always last. In this program, the US National Anthem came at the end of the program, but before India's national anthem. The 2017 program integrated the US National Anthem, right after Neerarum Kadaludutha, demonstrating that Tamil Nadu is highlighted first, the US is highlighted second, and India third. This order is quite consistent in the 15 programs that I attended. Regional identity takes precedent over national identities. The regional scale of Tamil Nadu is emphasized the most throughout the programs - through language, performances, visuals, and events celebrated. The area of Northeast Ohio is also highlighted through connections to Cleveland throughout the program. American national identity is highlighted second, and India third. These are represented as very separate, hierarchical scales with regional scale highlighted as most important - both through the emphasis on Northeast Ohio and Tamil Nadu.

Beyond highlighting regional identity, local scale is evident. Throughout the program, many skits were organized to tell a story of Pongal, a Harvest festival described as important to Tamil Nadu. The characters, second generation children in the skit, are played by second generation children and adorned in flashy leggings, mickey mouse purses, hats, 
sunglasses, Nike hoodies, and some armed with slow, few-word-sentences in Tamil. They follow an elder or mama (Tamil for non-paternal uncle) into the village to learn about the true origins of Pongal. True Tamil celebration of the festival is emphasized through the scale of the village. The village signifies the local, which becomes the most "authentic" and purest version of Tamil. The festival, of course, celebrates the harvest season which does establish the importance of farmers and people who work the land. The village participants, acted by some of the second generation children with a better command of Tamil, as well as their parents, are all decorated in traditional "village" attire, lungis and silk, Kanjivarum saris, as they partake in the 4 days, Bhogi, Pongal, Mattu, Kanum, of Pongal activities - Boiling milk over in a pot, racing and decorating the cow, drawing kolam (paintings from rice or chalk powder also called rangoli in other parts of India) and other festivities.

The skit also has implications for hybridity. It shows these two cultures as binaries, yet also in-between. The space that the children inhabit is in-between - they are represented as struggling to fit in-between two binaries - that of the purest local, village Tamil identity, and that of their American identity - mickey mouse, Nike, and accents. These binaries are reinforced further as the mama compares Pongal to Thanksgiving as a festival of harvest (this comparison in itself is really interesting) and tells the children things like, "in America you eat burgers and pizza, here in India we do not eat cows." This reifies the differences between Tamil and American identities. ${ }^{16}$ Food and language are used to demonstrate how these identities are opposed to one another. Everything is in

\footnotetext{
${ }^{16}$ With this particular example, though not in all, it also reifies that India is Hindu in some respects. Muslims and Christians eat beef.
} 
Tamil except for "burgers and pizza". The skit reinforces binaries between Tamil and American, suggesting that participants cannot occupy both identities. Yet, simultaneously, and somewhat contradictory, the overall program emphasizes that these identities are hybrid and both Tamil and American through inclusion of English and the US National Anthem. It suggests that Tamils in the US are Tamil and American.

These events are also emphasized as Indian through references to India and inclusion of India's national anthem, Jana Gana Mana. English words appear throughout the skit, but Hindi never does. Both Hindi and English are India's national languages, but scholars like Velayutham (2008) have described how Hindi dominates narratives of India. Nevertheless, references to India are made and being Indian is emphasized. But, these references are largely defined by a Tamil lens of India. Much of the program uses the regional scale of Tamil to emphasize India through Tamil Nadu and Tamil Nadu's history throughout the program. Yet, it also employs multiple scales of Tamil Nadu, India, Northeast Ohio, and the US - making it hierarchical, multi-scalar, and even hybrid.

In other NEOTS programs like Deepavali, a festival celebrated in other parts of India as well, is again defined through the regional lens of Tamil. Tamil is mobilized to specifically cultivate and define Indian Tamil in the United States. Distinction between Sri Lanka and Tamil Nadu is not verbally addressed, but is present with choice in national anthems, popular culture references, and ways in which festivals are described. For example, while the events are generally highlight through the regional lens of Tamil, they also incorporate the national lens of India to make distinctions between Sri Lanka and Tamil Nadu. The politics of these events is very layered. 
The display of identities at events beyond Pongal also reflect colonial notions of hybridity. For example, India is referenced as a place where original festivals happen where Pongal, Deepavali, or others are authentically practiced. Opposition between Indian and Tamil is silently highlighted, while opposition between Indian, Tamil, and American is more visibly and forcefully highlighted. Second generation children are struggling on stage for all to see with navigating these identities - being Indian Tamil as opposed to American, yet still connected through a diasporic experience and showing these identities exist simultaneously. These hybrid identities are also scaled, defined by one another, and inform one another. For the overall program, there is a purposeful mobilization of scale to define Indian as Tamil, yet not focus on broader Indian festivals, but rather on Tamil. Separately, there is a purposeful mobilization of specific identities to be opposed to one another in the skit, yet to an observer, hybridity is quite visible. Children struggle to fit into these seemingly separate but connected identities that are being represented as authentic or pure, when in reality, the very program is demonstrating that identities are hybrid, connected, and inform one another.

\section{Hybrid and scalar Indian vs. Tamil in American context}

Scale, as evidenced in the above program, is hierarchical, with traditional ideas of regional and national emphasized, often with regional taking precedent over national. But, these scales are also multiple as they are weaved in and out of the program, depending on moment and context. Hybridity is demonstrated in that identities are represented throughout the program as being separate, much like what is emphasized through colonial binaries, yet they also exist in spaces of in-between and are hyphenated. 
These identities and scales are not just confined to context of India. This distinction serves as a resistance in the United States as well. For many participants, like Raj in the introduction, "Americans know little of the geography of India." Many, if not most participants described that they felt this way in the US. They felt that this was evident not only with people they interacted with, but also with broader representations of Indians in the US, further discussed in Chapter 7.

Some participants not only described Indian as a broad scale that marginalizes Tamil identity, but also a broader statement or means to marginalize non-white people in the US. Indian, for them, was a homogenized category that erased historical nuance of people in the US. Sakhti, born in Tamil Nadu, but raised in the US says she does not like the term Indian. She says that it takes away from being Tamil and it does not really make sense for her. "I kinda feel like being called Indian, it sounds almost generic to me. Ok, you identified me as a brown person. I feel like being from a different part of India - most people that people run into - it is not the same thing or the same experience. I feel like people don't realize that. They are like, 'you are Indian you must know about that' (referring to popular Indian stereotypes). I'm like 'no."' She then explained that her experiences are very different than her North Indian friends. So even though she could identify nationally as Indian like her friends, their experiences would be vastly different.

Anusha, also born in the US, expressed similar sentiments as Sakhti regarding Indian identity. “Um, I know I’m supposed to, but I don't really, honestly. Sometimes I do. I much, much more identify with being Tamil than being Indian. If someone were to politely ask me if I'm Indian, I'm sure I would say yes, but it not how I would describe myself." She also 
describes her aversion to, or arguably rejection of Indian as being related to the way that people homogenize Indian identities.

I think part my resistance to it and what I do associate with it is the idea that as a country it is a homogenous thing. For me, my resistance to it is how people in America can lump me into a category or associate all these things that have nothing to do with me. I associate like Bollywood and Hindi, and Hinduism with being Indian, even though I know that is not really accurate, the generic things that Americans associate with being Indian - things that don't feel relevant or related to my experience like chana masala. I feel like I don't have a resistance to being Indian but being identified with Indian is like an oversight of what I associate with being on the subcontinent.

The national scale of Indian erases regional distinctions of Tamil for some in the context of living in the US society, not just with experiences in Indian communities. For others, it follows what Nicely (2009) described as scales informing, influencing one another, and being multi-layered. Indian identity is a threat for some, and for others, it is simply problematic. Kumaran was born in India but grew up in the US. His mom, Lakshmi, who I also interviewed, was visiting family in India when he was born. Kumaran had a few different views on the relationship between Indian and Tamil than his mother. In Kumaran's descriptions, these typical scales of national or regional are not always opposed, but often inform one another as Nicely (2009) stresses. He also does not view these scales as the typical national/regional hierarchical divide, but rather a scalar divide of culture and 
language situated by a specific history. But, that history is not as close to his identity as it is his mother's. He is another generation removed from the colonial struggle and grew up in an entirely different setting.

Colonialism was directly relevant to his grandfather, who fought for India's independence. At that time, as Devdas and Velayutham (2008) indicate, Tamil Nadu promoted a concept through state-sponsored media, films, cinema, newspapers, and other forms of discourse to spread the message of unified Indian. This was promoted as a means of resistance to British colonialism. At the time, the direct threat was the British empire, and thus, Tamil Nadu scaled up in representation to promote the concept of a national Indian, representing Indian as being opposed to other nationalities (Devadas, 2008). It was after independence, that Tamil Nadu began to see North Indian domination and the Hindi language as a threat (Devadas, 2008). Thus, the regional scale of Tamil Nadu was promoted again through various state-sponsored media described above. Though the regionalistic fight against Hindi mellowed in many ways, it surfaced again with Prime Minister Narendra Modi's policies to push Hindi in many parts of India and in official government proceedings (Kalra \& Asokan, 2014) . Lakshmi's identity was situated by her father's struggle during and after colonialism, which she grew up hearing. She was also born and living in India shortly after independence. Kumaran, however, lived much of his life in the United States and knew a postcolonial India much later after independence, so he was far removed from any of these factors.

Kumaran's hybrid identity is American, Indian, and Tamil and these sometimes blur. For Kumaran, national Indian and regional Tamil can sometimes be used interchangeably, especially in relation to his American identity, but, as with most hybrid identities, it often 
depends on the context. He does differentiate between North and South India, giving meaning to these subnational scales, but in terms of hybridity, constructs the idea of being Indian through his experiences as Tamil. He identifies with a national scale of American but demonstrates that the national scale of Indian informs the regional scale of Tamil. He says, "So I think of Tamil as Indian. I knew when I was younger that the Southern Indian language are related and different from Northern languages. I had Northern Indian friends and I knew all of these things are Indian. I didn't know Hindi, but I knew English and Tamil." He said though, that despite this, Indian was a difficult identity to maintain. He expresses similar sentiments regarding Indian as Sakthi or Anusha regarding Indian being used as a homogenous category to erase nuance in the US by Americans, Indians, and Tamils, often leading to stereotypes or discrimination. In the context of the US, he says, "I recognize that Indian itself doesn't make any sense. There is no Indian. People would ask, do you speak Indian? You have to explain that there are a bunch of languages." When he thinks of Indian, he doesn't often think of Hindi even though it is the national language, but rather Tamil. He defines Indian through the regional lens of Tamil, rather than a national lens of Indian. He does not conceptualize this scalar relationship in the same way as Lakshmi or even Arvind. In fact, he says he has always been American, Tamil, and Indian, but defines Indian based on his experiences as a Tamil-American. Unlike how some participants felt forced to choose between identities, Kumaran, though he says that he has definitely been forced into that experience multiple times, in general, describes that all of his identities inform the others. Yet, while he described this personal experience, he does note that these identities are represented by societies in specific ways - often as 
separate and distinct, which makes it difficult to identify when these identities often blur and overlap.

While Kumaran described some difficulties in navigating these identities, some participants did not make a distinction between being Indian and being Tamil at all. Vikram, for example, said that they are the same for him because being Indian really meant describing where his family came from and Tamil, he thought of as more of a language than a culture. He did not make a scalar distinction between Indian and Tamil, because he did not address these identities as scalar concepts. In terms of hybridity, he described himself as Indian, which he elaborated really meant Tamil or hyphenated Indian-Tamil. He acknowledged that other Indians have different experiences but suggested that it is based on individuals rather than some grand distinction. Overall, he said he felt more American anyway.

His sister, Sundari, also said that being Indian referred to someone "who grew up there." So like Vikram, Sundari attached place to the meaning of Indian. Unlike Vikram, however, she definitely made a distinction between being Indian and Tamil. Her definitions of these two categories that she separated found deeper grounding in hybridity. For Sundari, these identities were related and dependent upon one another. India was indeed a place where parents were from, but they were also Tamil. Sundari said that she was Indian because of her parents, but simultaneously not really Indian. Tamil was what she described as her "cultural identity." India was just a place, not really related to the way that she felt about her identity. It was ascribed to her through her experience of living in the US. In other words, she had to scale up to identify as Indian with people who had no knowledge of India's diversity. 
Participant responses varied significantly based on emotions and experience. For many participants identities were scalar - either hierarchical and/or multiple, but they were also hybrid and interconnected. Indian and Tamil were conceptualized as scalar in the sense that they are either broader, large-scale identities like Indian or used interchangeable with South Asian or desi (and at times even "brown"), or small-scale identities that focused on regional Tamil, or even specifics like villages or towns. These identities were co-constructed as Johnson and Coleman (2012) suggest, and purposefully mobilized as Marston (2000) indicates, for specific purposes - either to cultivate unity or prevent erasure. But yet, these identities were very much hybrid, and many viewed themselves as Indian-Tamil or Tamil-Indian, suggesting that both parts were blurred together or that Indian and American, American and South Asian, coexist and build off of one another. Others described they were in-between identities, making them hybrid identities. Yet still, as I will discuss in Chapter 8, identities were also hybrid products of mimicry and contradiction where identities mimicked colonial behavior, creating tensions between whiteness and blackness. In other words, there was a tendency to mimic colonial binaries of white and black/non-white where to fit in, one must mimic whiteness.

For example, some participants actively described Indian as a "safe" category in the US, much safer than some of their other identities. Essentially, Indian was closer to whiteness. As critical race scholars and geographers like Dyer (1997), Delgado and Stefancic (2017), and Bonds and Inwood (2016) suggest, the default norm is whiteness. For Nazeem, and many others, Indian is closer to whiteness than Pakistani. I discuss the links between Indian and whiteness further in Chapter 8. For Nazeem, identifying as Indian is safer than identifying as Pakistani: 
Being Indian and Pakistani to me didn't make much of difference because I was here. For where it matters, I would probably identify with being Pakistani more, but here, it plays more into politics. People (in the US) think they understand Indian better. Because if you say you are Pakistani, they consider you more from the Middle East. It is easier to say I'm Indian than Pakistani. Among Indians, it makes a difference too. She describes Islamophobia in both American and Indian communities as a source of concern for her, which is why she will sometimes strategically place Indian at the forefront of her other identities. Because as Nazeem and other participants indicated, Americans don't think of Indians as Muslims. This is further discussed in Chapter 7.

For others who also grew up in the US, defining themselves as hybrid - Indian in conjunction with American creates a safe identity to build connections. Durga, for example, sees Indian as a hyphenated identity with American. Indian and American created a unique view of being Indian that was important to establishing solidarity in her life experiences. She views identity somewhat in way that Papastergiasdis (2015) describes hybridity as "cultural multiplicity." She sees Indian and American as intertwined and an experience that cannot be separated. She describes this identity as, "shared sense of values. With other Indian-American people, I don't have to explain. Just feels like there is a short hand to that." She says that she is not just Indian and not just American, but both. With people are are strictly Indian or American, she has to explain too much, but other "Indian-Americans" just get her. In other words, Durga sees a blurred hybridity as key to her identity. 
For Durga, Indian is very different than Indian-American. She says this is because the experiences of growing up are different as well as world views. Indian-Americans have a shared experience, while people from India do not really share that same experience. Likewise, explaining also comes with trying to identify as American. For Durga, her experience is separate, but hybrid. In many ways, she rejects the notion that she is forced to fit into binaries. She also attributes this the places that she has lived, noting that most of them were very diverse and urban. Other participants, who grew up in rural or homogenous areas with lack of diversity, mentioned that they did not have the agency to assert their identity in such a way. This was quite significant, and I discuss this further in Chapter 7. This was especially true if they did not grow up around other Indians or IndianAmericans. Again, place, context, and situation were important.

Sakthi's sister, Vimala, who grew up in the US, describes herself even more broadly as South Asian and American, like Nazeem. Though she sees them as more of a "dichotomy" of hybridity rather than a connected experience. She says that she is always struggling to fit into both, especially with the tensions between what she sees as "individualistic in American culture" and "collectivist in South Asian culture." Vimala defines each identity by comparing them to one another. Initially, she describes South Asia in opposition to American and American to South Asian. But, this changes a bit when discussing discrimination, which I will elaborate on in Chapter 6. Overall, South Asian incorporates is Indian, Tamil, and Hindu for Vimala.

The meaning for me in that is that I was born and raised here, in the US as a person of South Asian descent. If you had asked me when I was younger, I probably 
wouldn't have said that then, not that I wasn't, there was just a lot of bullying and things (she referred to bullying that happened to her in school by her peers, often white). My research now focuses on intimate partner violence on South Asian women. As I've gotten older, I've become more connected to some of those cultural values. I also feel that when I identify myself as South Asian, I connect to my family here and in India. I don't separate the whole South Asia culture from being Tamil or being a Hindu.

Indian and Tamil were hyphenated with being American for many or defined in opposition to one another. Living in the US has influenced many identities, but American identity is not always seen as a hybrid identity with Indian or Tamil. In the 2016 Pongal festival, American was represented as opposed to being Tamil at times and connected to it in others. For some participants, though, American identity was not just defined in opposition to Indian and Tamil but was a threat to Indian and Tamil identities.

Diya, for example, who advocates identifying nationally as Indian first, says that Indian identity is important because it means having a common culture even with diversity, and this common culture is centered around family values. I asked her to elaborate and she said:

I don't see that kind of upbringing in the culture here. It is completely different. When you see Indian, it is automatically so different from others. I grew up in a principled manner. That helped me to be a better person. Because of that... I'm not sure here. It is very loose here. I don't see that kind of - like for example, the 
sibling's affection, the way you look at taking care of your parents, family relationships. You are always responsible for yourself and others. That is very important to me.

She says that other parts of India have these same values and yet she does not see that in the US. Though she has lived here, this is why she does not identify as American. Others, even those who had been here a while or grew up in the US, expressed similar sentiments.

Many who expressed that these identities were in opposition identified as American instead of Indian or Tamil. Matthew, who came to the US from Tamil Nadu when he was nine years old and grew up in Alabama describes himself first as with the national scale of American, but then as Indian. This came up casually as I did not ask him to rank his identities. He says he only describes himself as Tamil when others ask what part of India he is from. But even being Indian, he says he feels much more American based on values he associates with being American. “My blood is Indian, and my family is Indian. My qualities/how I live is not really Indian. I cook Indian food, but I'm pretty limited as far as the things I do or how I feel that I'm Indian." His father was born in Myanmar and his mother in Sri Lanka, but he describes them as Tamil. They lived in Tamil Nadu for a good portion of their lives. I asked him what he meant by Indian values. He said mostly food, religious activities (though he is Christian, he often associates this with Hindu culture), and family values like spending lots of time with family. I asked why he felt more American and he described that he had more liberal, democratic views on social issues, though he also works in defense in what he describes as "geared toward the armed forces," so also holds America's defense as important to him as well. Mostly, he says, he has a lot of pride in being 
an American citizen. His narrative of being American did not necessarily contradict his narrative of being Indian, but yet he said he didn't feel that he was really Indian in the "traditional" way that has been represented to him, through family, films, media, throughout his life.

Indira, who came to the US in the late 60s, says she has adapted to being American. She describes American qualities as good, but yet still describes them as in opposition to being Indian or Tamil. While Indian and Tamil can be hybrid identities, American is just so different from these other identities. "I have noticed one thing in Americans, that they are giving, I want to absorb that quality from them. There are very good qualities we have to learn from Americans. Like when disaster comes - they don't think twice they just give to the people. That is the thing that comes first in my mind." She says that she is frustrated when Indians or Tamils try to segregate themselves from other living in "this country."

Ruth expressed similar sentiments as Indira. "America is very giving culture. Which we don't do in India. Especially Christians. It is difficult to give. I've learned a lot of good things about Americans. If they say yes - yes. If they no - no. For example, if they (Indians) say come home for dinner, I don't know if they mean it. But Americans mean it. I love that culture - that is very American." Many others described this as American, the idea of saying it "straight" or "telling it like you mean", while also being very giving and charitable. Yet, this is not a consensus. Many related being American to business-oriented or capitalistic, associated it with the destruction and disregard of the environment, and loss of nature. And others, described these same things of being Indian. Some said American was "being polite to your face but talking behind your back." But, for other participants, this wording was used to describe being Indian. 
So, American was associated with specific qualities that at times, were also used to describe "Indian" and vice versa. These identities overlapped and varied in participant definitions. Similar to Indian, American was also defined as nationality or citizenship often placed on participants or was associated with specific privilege or qualities. Many participants like Kalai, who grew up in the US, described American as a default identity associated with citizenship. American is her default and when she has to discuss it, it is usually because people are discriminating against her. "I assume I am American and I don't say it out loud. If I have to say I'm American, it is usually because people are threatening me. I'm being forced to self-identify." For many, American was their citizenship that came with many privileges. Many said that they could tell me what American meant for them, but they recognized that there were no specific values that all Americans shared. Many described American as opportunity.

Suhail, for example, sees American identity as connected to opportunity. "American, I have a lot of opportunities, resources. I identify as an American because it has given my family so much. I feel like I have a lot of resources compared to most people on this Earth." While for some like Suhail, being American and having opportunities was a source of pride, for others Anusha, it was not a comfortable identity. Anusha describes it as, "I don't know if it is necessarily an identity I'm proud of, but it is a huge privilege in a lot of contexts. To not identify with it would be disingenuous, and not accountable. I don't think I identify with being American in an affirmative, particularly proud way. But I know that it doesn't feel like that when I'm in American, but it situates my access and opportunities, so I want to be accountable." Anusha said that she is very aware of privilege and describes herself as “Tamil, woman, person of color, brown person, queer, gay, I think about identifying as like 
having socioeconomic advantage, I'm like educated in a pretty mainstream way, lawyer, social justice advocate." Her identities intersect in various ways that affect the way that she sees privilege.

For some, American and American ways of life threatened Indian culture in the US. For others embracing an American identity was a way integrate into US society. America was associated with "Western" or with being individualistic. Individualistic meant, for some, ignoring family values and for others, individualistic meant freedom. American also meant honesty and charity for some for others it wasn't just honesty, but it was a way to silence being Indian.

\section{Implications}

No one person described their identity or interactions with their identities in the same way as another. Like most people, my participants fluctuated between many different identities. Identity varied with individuals, yet many described a sense that Indian, Tamil, or American, were larger identities based on some of their commonalities. With most participants, at times, identities were scalar - often hierarchical, sometimes multi-scaled, and also hybrid. In terms of scale, some scales took precedent over others, depending on situation and context. For Indian and Tamil, these scalar tensions were characterized in many ways. For example, some participants suggested that identifying as Tamil was important to prevent being subsumed by Indian homogeneity. Yet, others described that Indian unity, was more important than divisive Tamil politics.

Indian and Tamil also began to be used somewhat interchangeably in some situations, in relation to American identity. For some, American sometimes overlapped 
with Indian, depending upon those defining. Essentially, most participants and some events suggested that there were qualitative differences between these identities, creating binaries and essentialized ideas about each of these identities. Yet, participant descriptions and event representations often contradicted the idea that these identities were homogenous and, in many cases, demonstrated incredibly fluidity and hybridity.

Indeed, identities are more than processes of hybridity and scale. But, I chose to focus on how these two concepts inform identities in this analysis precisely to highlight the complex, variegated nature of identity in the Indian Tamil diaspora in US. In these examples, hybridity and scale are important to understanding participants' identities. They are also important to understanding how identities are represented through events and performances and even in governments, media, academics. People live and experience identities that both reinforce and diverge from representations. People understand identities as hierarchical and traditionally scalar in some moments, but simultaneously experience diverse, blurred and hybrid identities in others.

In the next chapter, I use specific examples to illustrate how sound informs identity. Sound illustrates how people experience both hybridity and scale in everyday life. Sound can also identify how specific ideas about identities are part of a larger discourse, but they are equally important to providing everyday, embodied examples. Diasporic identity is complex, multiple, contradictory, and not singular and I will demonstrate this further in the next three chapters drawing on sound, discrimination in the US, and discrimination in Indian communities. Before addressing discrimination, however, I will first focus on sound as it provides countless insights into moments, memories, feelings, and nonrepresentational elements of identity. 


\section{Chapter 5: Sound and Identity}

Sound's importance to identity is a key part of this dissertation. Hybridity and scale lend crucial insights into identity, but I build on identity by also integrating sound into my analysis. This chapter focuses on the links between sound and identity. There is some overlap between sound, scale, and hybridity, especially in the sections on how various sounds reinforce or define fluid hybrid, scaled identities like Indian, Tamil, or American. The literature that I reviewed in Chapter 2, demonstrates the usefulness of sound to identity analysis and more specifically, to understand the experiences of Indian Tamils in the United States.

Before discussing the results of my data on sound, I want to address three points. First, much like with hybridity and scale, individuals had varying experiences and ideas about what sounds influenced identities and how sounds influenced identities. While individuals described some similar views on sound, I found that like hybridity and scale, these experiences and ideas changed based on situation and context.

Second, how specific sounds linked to identities like Indian, Tamil, or American, varied based on participants. For example, while multiple participants identified a particular sound, like the pouring of tea, they would associate it with different identities. Some would say it reminded them of being Tamil, others, being American, and so forth. Overall, most participants had very different views of what each sound signified or how it interacted with other identities. These ideas and experiences are not surprising, but as I demonstrate in this chapter, the meanings of these experiences are significant. Third, 
identity and sound intersect with discrimination in many ways. I address the bulk of data on discrimination in chapters 7 and 8 but introduce it in this chapter. I first provide an overview of identity and then demonstrate the importance of environmental sounds, music, language, and accent to identity before addressing discrimination in the subsequent chapters. I found that music, accent, language, and environmental sounds were significant to identity.

\section{Overall sound for Tamil Americans}

Music, accent, language, and environmental sounds were significant to participants' identities and also to events and performances. For events and performances, for example, facilitators of all of the Northeast Ohio Tamil Sangam (NEOTS) events spoke in Tamil and used Tamil script throughout. In the NEOTS events, music was often in Tamil and environmental sounds such as Bharatanatyam bells or anklets permeated the atmosphere. As described in the last Chapter, accent was important to how Tamil identity was represented in performances. For example, the Pongal skit represented some accents as more authentic than others. Specific Tamil accent associated with more local scales like Madurai or Chennai were not necessarily highlighted, but for example, Madurai was often referenced as having the purest Tamil. For a 2016 Deepavali ${ }^{17}$ event, a comedian from Chennai did perform a skit that highlighted different accents in Tamil Nadu but did not make an overall commentary on accents. He simply identified that they were different. For the most part, accent was acknowledged, but the idea of a universal, united Tamil Nadu

17 Deepavali is the South Indian equivalent of Diwale, the Hindu festival of lights. Though, many religions celebrate Deepavali, not just Hindus. 
seemed to be dominant. Much spoken Tamil at events in the US was often mixed with English. Events in New Jersey also mirrored these events in use of language, music, and environmental sounds. I will delve into specifics later in the chapter, I want to show that overall, sound was integral to these events.

While participants described sound in their interviews, they also indicated sounds' importance in the Likert scale rating after the interview (see Appendix A). Most participants who answered this question indicated that sound was quite significant to their identity. Participants rated sound's importance to their identities from a 1-5 on a Likert scale. Responses ranged from 1-5, some even suggesting 7s and 8s. When I explained that the numbers stopped at 5, these individuals stressed that they marked 7 or 8 because they wanted to demonstrate that sound was extremely important to their identity. Out of 39 participants who answered the question, the average response was 3.97 out of 5 . I did not include 7 or 8 , but rather placed that number as a 5 . The median response was 4 out of 5 .

Sound was temporal and changed throughout individual lifetimes. Some individuals said sound was more important during certain periods of their lives than others. One participant, Sam ${ }^{18}$, described how the loss of hearing changed his relationship with sound. This has impacted the ways in which he views sound in relation to his identity. I asked him how important sound is to his identity on a scale of $1-5$. He responded:

I will be biased regarding this question. If you had asked me this question 6 or 7 years ago, my answer would have been very different. In the last few years my

18 Sam's interview was one of the only interviews that I conducted via electronic communication, because of his hearing loss. 
hearing loss is so severe that even with the hearing aid the sound is not as crisp or vivid. I used to have surround sound. I have many speakers one time in my home and used to record with my pro stereo recorder. Today I would rate it 3. Sound has always been very important to me. I love universal music and I find that music is a universal language just like photography. I used to record almost all sounds with my professional recorder. Birds singing to crashing waves in the ocean. During my extensive travels, I used to record local music in all parts of the world. I would (have) rate(d) at 5 out of 5 .

Sam's views on sounds changed significantly because of his hearing loss. Many scholars have written on sound and identity's importance for hearing-impaired in a visually-centered world (Smith, 2000). Focus on visuals can exclude people who are visually impaired or blind (Smith, 1994). These individuals often rely on sound or other senses to understand the world and visual-centered research can ignore these voices (Smith, 2000). Yet, at the same time, as Sam's story demonstrates, focus on sound can mask those who are hearing-impaired. It is important for researchers to value multiple senses as not to exclude those who do not rely on singular senses to understand the world. Relying on multiple senses exposes rich and diverse experiences.

Overall, sound was extremely important to understanding hybrid and scalar identities in both participant observation and interviews. In the next few sections, I describe how sound connects to identity. I break up sections into environmental sounds (including links to food and memories), music, language, and accent for clarity. First, I overview sound's links to Tamil identity as described in various events and interviews. 


\section{Sound and Tamil Identity}

Many participants described the importance of sound (music, accent, language, environmental sounds) to defining identities like Indian, Tamil, and American, but most often connected sound to Tamil identity. Some participants said that they did not think of sound with regard to American identity. Others mentioned this of Indian identity, though less often than with American identity. Though responses varied with regard to American and Indian identities, most participants agreed that sound was crucial to Tamil identity.

In many interviews, participants connected language, music, and sound to Tamil identity through literature. I did not find this connection as emphasized with regard to other identities that participants described. They discussed how literature and Tamil identity were connected to the history of Tamil Nadu through sound. Thus, when discussing literature, many participants connected it to sound. At least half of participants referenced the incredibly long and complex history of Tamil Nadu. Though, it is important to note that Tamil has not always been conceptualized by its boundaries and territories of today and was once part of a much larger geographical body known as Tamiliham or TamilNad ${ }^{19}$ (Selby \& Peterson, 2008).

Tamil Nadu's history is contested and often vague, and while many scholars argue that it can be traced back as far as 300,000 years, most accounts start at around 300 BCE (Kulke \& Rothermund, 2016; Sastri, 1975). Scholars have long suggested that Tamil Nadu's history is shaped by its geographic isolation from North India and its cultural and structural influences (Kalidos, 1976; Kulke \& Rothermund, 2016; Selby \& Peterson, 2008).

${ }_{19}$ Many participants still refer to Tamil Nadu as TamilNad. 
What many participants and programs like the Tamil language school, Tamil Malar Manram Kalvikkoodam (TMM), note is that sound is key to Tamil Nadu's history and is referenced in classic texts and literature, including the Thirukural, Silapathikaram, or Tolkappiyam, as early as 300 BCE. Songs, poetry, rhythm, and language were also linked to the physical landscape in many ways - architecture used tala (rhythm) to purposefully mimic musical structure (Sthapati, 2008). One participant sent me a few links to her favorite temples that use the musical structure in construction. These included famous Tamil Nadu temples such as Nellaiappar Temple in Tirunelveli, the Meenakshi Temple in Madurai, and the Alwar Tirunagari Temple in Alwar Tirunagari ${ }^{20}$. In Tamil Nadu, there are countless temples linked to sound and have been constructed through a variety of rulers and dynasties (Sthapathi, 2008).

As briefly mentioned in Chapter 5, of particular importance to many participants and often the foundation of Tamil Sangams in the US is the Sangam Age, from approximately 300 BCE to 300 ACE (Kalidos 1976; Selby 2008). Not mentioned in the previous chapter, but relevant to theme of this Chapter, are connections of Sangam culture to sound. Sangam culture idealized the trinity of iyal (poetry), isai (music), and natakam (dance) (Kalidos 1976). The Sangam Age is credited for establishing the classics of Tamil literature and described as crucial to Tamil history, with Tamil Sangams in the United States following these traditions to "educate future generations" (Chattopadhyaya, 2008). Much of the literature mentioned above is used in instruction for Tamil schools or was referenced by participants as part of the historic, emotional, and poetic sound

\footnotetext{
20 This temple was constructed during the reign of the Pandya kings, a different historical period of Tamil Nadu than the Sangam period, but is also equally important to the links between sound and Tamil identity.
} 
connections to Tamil identity. For example, the Tolkappiyam, one of the famous literary grammatical works in Tamil history, outlines tinai, the association of geographical lands and features with specific behaviors; akam, the interior themes, often love; and puram, the exterior themes of non-love (Ramanujan, 1967; Rathinasabapathy, 2008; Selby \& Peterson, 2008). The significance of tinai is not that it embodied five geographical regions, though. It is significant because each with its own form of svarams (musical notes) and instruments that connect to these larger themes and geographical areas, thus intricately connecting to sound (Kalidos, 1976; Kulke \& Rothermund, 2016; Manuel, 1997; Ramanujan, 1967; Selby \& Peterson, 2008).

Sangam and post-Sangam literature connect to sound in many more ways (Manuel, 1997). For example, poetry, according to the Manuel's (1997) interpretation of the Tolkappiyam, is outlined as structure, marapu (tradition), valakku (content), implication and medium (language emphasis) is often one of the most important of the five. These five components create Tamil poetry - complex with many meanings and relationships related to sound (Manuel, 1997). Many references to sound appear integrated into the poetry of the Sangam Age. These elements place importance on sound and its qualities. Manuel (1997, p. 81) stresses the importance of the sound in literature, highlighting some of the components such as cerivu/cilttam - density of words and similar sounds; olukicai - harsh, sweet, or soft sounds; and camanilai - mixing of sounds. The Tolkappiyam is not the only referenced Sangam text that highlights sound. Another Tamil literary work, the Silappadikaram also known as The Anklet Story, describes many intricacies of not only the sound, but also dance and culture. Participants who identified literature as important to 
sound and Tamil identity did not always reference these items directly, but many knew that they existed, and they were integral to the historical connections of Tamil Nadu and sound.

While these works are not the focus of this research, I outline them because many participants referenced them, often when describing that sound is intricately connected to Tamil identity through these items. So, to some, these were extremely important to linking sound to identity. In Tamil Sangam events, students often recited ancient poems from classic literature texts like Thirukkural. Also, in the Northeast Ohio Tamil school that I attended, students were taught ancient Tamil poems to learn the sounds and literary text of old Tamil. Students start by learning Aathichudi, written by the famous poet, Avvaiyar ${ }^{21}$, who starts all of her lines with each of 247 letters of the Tamil alphabet. The importance of this poem is two-fold. First, it teaches children the sounds of ancient Tamil - tones, alphabets, inflections. Second, each line teaches students good deeds along with sound. The first line, "Aram seiya virumbu" roughly translates to "have intent to do good deeds". The second line, "aaruvathu sinam" translates to "reduce anger", and so forth for 247 lines. Tamil teachers at TMM, described in the classroom that learning Tamil was both learning the sounds of Tamil, but also connected sounds to learning how to be a good person. In these instances, sounds - language, poetic diction, and quality of Tamil sounds are associated with specific behaviors. Tamil is a language, it is a sound, but it also denotes Tamil identity, and more specifically, positive qualities of Tamil identity.

Essentially, sound was intertwined and crucial to learning, practice, and performance of Tamil identity. Tamil schools were one way that Tamil communities in the

21 There are actually multiple Avvaiyars, the most famous of them existed during the Sangam Age and the $10^{\text {th }}$ century during the Chola dynasty. This Avviyar is the second Avviyar, but people often just refer to Avviyar as one Avviyar who became sort of a legend that lives on. 
US emphasized Tamil identity in children through sound. More specifically, sound was intricate to the passing on of Tamil identity. Many participants I interviewed from Northeast Ohio and New Jersey sent their children to Tamil school. Participants in Morgantown, though they did not send their children to Tamil school, described that they wished that Morgantown had resources to create a Tamil school, so they could learn how to properly speak Tamil.

Tamil school represented Tamil as more than just a language, but rather an integral part of an identity through language and sound. Learning ancient texts and specific sounds were important to learning qualities of being a good human, but also denoted specific differences from other Indian languages. Tamil was discussed as one of the oldest, most ancient languages and described as separate from any North Indian languages. These differences between Tamil and other Indian languages were stressed. The official textbooks came from the state of Tamil Nadu so the context of stories, reading, and language was situated in Tamil Nadu. Much like the examples in the last chapter, the regional lens of Tamil was purposefully mobilized through language to define Tamil as separate from Sri Lanka as Tamil was associated with the national scale of Indian, but also to highlight Tamil as a regional scale and demonstrate that Tamil is different or more unique than other parts of India.

Though Tamil instruction was important to many participants, often, those in the second generation that I interviewed never had opportunities or desires to attend Tamil school. Even first generation participants did not attend Tamil school in the US, but rather volunteered or sent their children to school. Two participants I interviewed, both second generation, actually attended Tamil school and only one of them attended continuously. 
Additionally, not all participants had knowledge of these texts, histories, or background information. For some participants, the response to sound was much more visceral. These links were established through memories, feelings, and familial connections rather than a deep tie to historical Tamil Nadu.

In fact, almost all of the participants connected to what they described as American identity and Indian identity often in a more visceral way than how many connected to Tamil. For example, many described Indian identity with sounds of family members, this was often cooking. They also described Indian, American, or Tamil with some Bollywood, Kollywood, or American pop song their parents of family played for them via movie or audio when they were young or that they remember from school. Connections to music were prominent with many described identities and often linked to memory. Rarely, were references to Indian or American associated with history or literature emphasized in the way that participants referenced Tamil literature. Although, a few participants did reference India's religious texts, the history of the Vedas, Sanskrit proverbs, and India's rich cultural history. One participant referenced American documents like the Declaration of Independence, referring to themselves as a "history nerd." But, overall, the links to Tamil history and its connections to sound were much more pronounced.

In the next few sections, I provide further examples of sound's links to identity. For the purpose of clarity, I have broken sound into four main sections - environmental sounds, which also includes a discussion of memory and food and sound, music, language, and finally accent. Accent leads into the discussion and subsequent chapters on discrimination. This chapter focuses on how sound informs identity, while the subsequent chapters address how discrimination overlaps with sound, hybridity, and scale. I have 
provided a table (see Table 1) and figure (see Figure 5) for the next few sections to demonstrate how sound intersects with some of the spatial identities described in Chapter 4. Participants often linked specific sounds to spatial identities as shown in the table.

Table 1: Sounds and various identities

\begin{tabular}{|l|l|}
\hline \multicolumn{1}{|c|}{ Identity } & \\
\hline home & Environmental sounds \\
& Music \\
& language \\
\hline village & Accent \\
& music \\
\hline city & Accent \\
& Environmental sounds \\
\hline County/district & Accent \\
\hline Northern/Southern Tamil Nadu or US & Accent \\
\hline Tamil - Tamil Nadu & Language \\
\hline Tamil - Historical Tamil Nadu & Music \\
\hline Indian Tamil & Language \\
& Music \\
\hline US State & Language \\
\hline South Indian & Music \\
& Accent \\
\hline Dravidian & Accent \\
\hline Nation - India/Indian & Music \\
& Language \\
\hline Indian American & Language \\
\hline Tamil American & Music \\
\hline Nation - US/American & Language \\
\hline & Environmental Sounds \\
Accent \\
Music \\
\hline & Accent \\
\hline Environmental Sounds \\
Music \\
\hline & Language \\
\hline Environmental Sounds \\
Music \\
\hline & Environmental Sounds \\
Music \\
Accent \\
\hline
\end{tabular}




\begin{tabular}{|c|c|}
\hline South Asian & $\begin{array}{l}\text { Environmental Sounds } \\
\text { Accent } \\
\text { Music }\end{array}$ \\
\hline desi & $\begin{array}{l}\text { Environmental Sounds } \\
\text { Accent } \\
\text { Music }\end{array}$ \\
\hline brown & $\begin{array}{l}\text { Environmental Sounds } \\
\text { Accent } \\
\text { Music } \\
\text { Language }\end{array}$ \\
\hline Global citizen/human & Music \\
\hline Part of the universe & --- \\
\hline
\end{tabular}

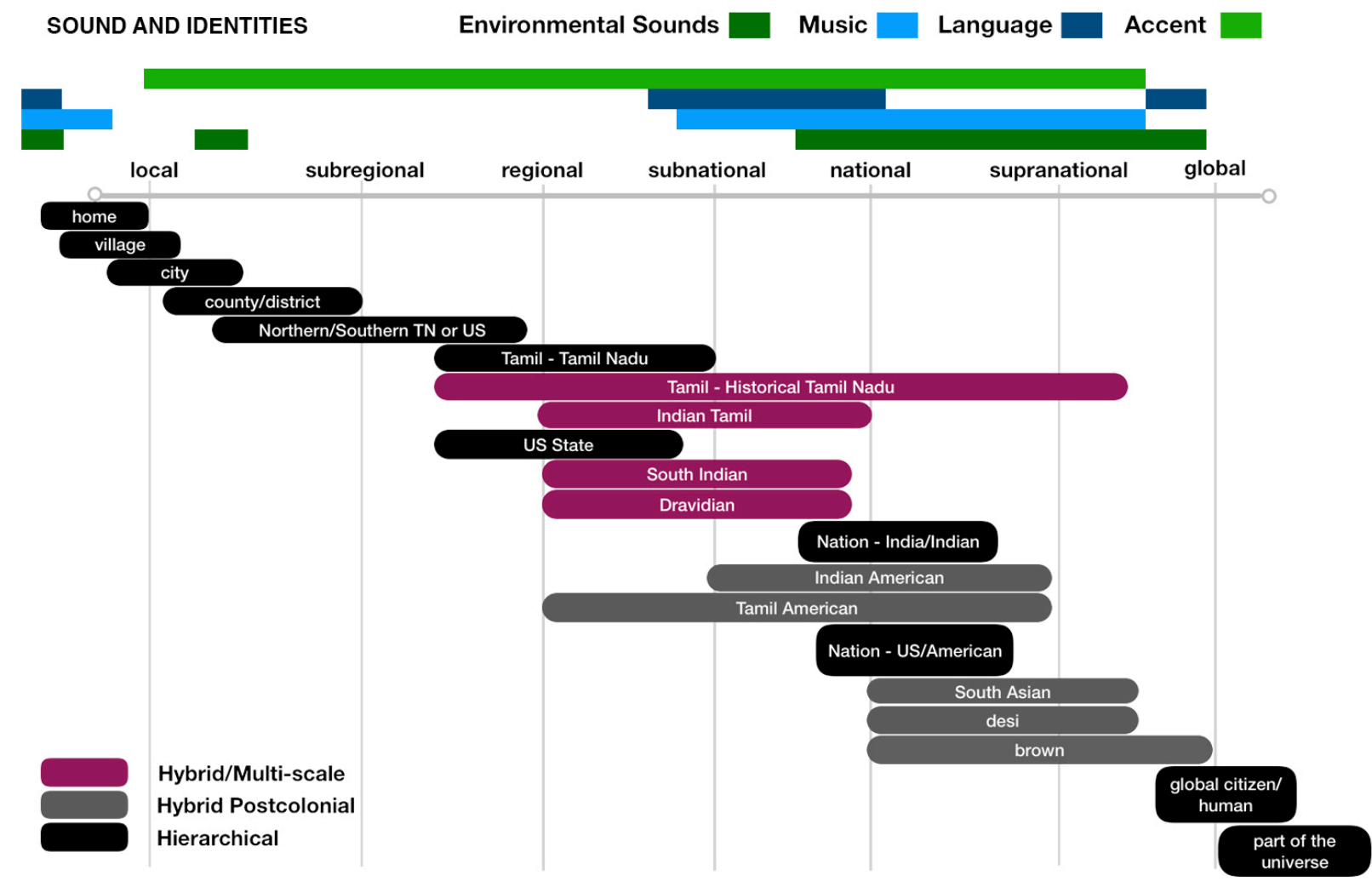

Figure 4: A visual demonstration of how sounds intersect with identities. 


\section{Environmental Sounds}

While soundscapes are all encompassing and include items like music, accent, and language, soundscape researchers generally categorize environmental sounds as the sounds that make up the background of an environment (Pijanowski et al, 2011). Essentially, many researchers have used this term to encompass the background noises that contribute to specific environments, memories, or situations. Often, when scholars refer to the term 'soundscapes,' environmental sounds are often what they highlight (Smith, 2000). As Miller (2007) explains, they are noises that permeate a specific environment and are recognized aurally. Southworth (1969) is often credited for identifying environmental soundscapes as an important element of the urban landscape. What he refers to as a "sonic sign," or the awareness of a sound, creates visual images in the viewers' mind that reinforce an image that they see through sound that they hear (Southworth, 1969). For example, imagine coffee brewing - you see the coffee dripping into the pot and you smell the aroma of a fresh grind. You also associate the sound - perhaps the sputters and whistles of coffee pot and, depending on the pot, or perhaps the sounds of droplets plopping into the liquid. Then, you imagine the next sequence of events - the coffee pouring into a ceramic mug as it swirls around the edges, finally settling into a calm mass of liquid ready to drink. Environmental sounds are connected to other senses, memories, and actions and much like other sounds, they create a multi-sensory experience (Kapchan, 2016; Waterman, 2006).

Throughout my observational experiences, environmental sounds were key to interactions, events, and performances. These sounds overlap, of course, with music, language, and accent, but also remain distinct in some ways. In many events and 
performances, the sounds of silk sarees or dhotis, clinking bangles, anklet bells, clapping, rhythmic clapping for music, bustling conversation in Tamil rebounds from multiple chattering groups. Yet, many of these sounds might be reflected in other Indian events as well. These are not clearly Tamil sounds, but rather sounds that make up a specific environment - in this case, an Indian musical gathering. Tamil tones in language, however, are identifiable to those familiar with the language as they are complex, heavy in the mouth, and ripple across vowels and retroflex consonants. While these events that I attended were specifically Indian and Tamil, environmental sounds are not unique to Indian or Tamil events, but also permeate American landscapes.

Environmental sounds in general, surface in everyday, banal ways - perhaps through a coffee pot brewing or soft or booming chatter of conversations in a university hallway, the inflection, tonality, and accent of broadcast news anchors speaking in the American Standard version of English, yesteryear's refrain of Elton John's “Your Song” echoing through a grocery store, muffled engines of traffic, coats, boots, and winter clothes shuffling and sweeping across a university floor, a fan whipping through a window while birds chirp and a lawnmower roars - and also in more augmented, ritualized ways. Imagine a winter evening in Northeast Ohio, where Americans cross towns and join friends to swarm into barrooms or living rooms for an annual, ritualized event. The fluctuating and muffled crowd noises emanate from the television, while crinkling bags, harsh and chattering voices, crunching chips, and frustrated yells at the TV permeate the atmosphere. Soon, the National Football League theme song plays, signaling the commercial break of the Super Bowl. For some, these sounds elicit positive feelings or memories, while for others, they can be irritating or crass. These are a few examples of how sound permeates 
environments. But, the significance of sound has a variety of meanings regarding scale, identity, and discrimination.

Sound's significance varies from person to person and can be produced in augmented ritualized ways like the American Super Bowl Sunday or through formal performances of South Indian Music at the Cleveland Thyagaraja Festival held at Cleveland State University in $\mathrm{OH}$. Notably, despite how they are enacted or encountered, environmental sounds abound in various facets of life. Also, notably, while some sounds are associated with specific identities - like American football or Karnatak music, these sounds are contested and can represent hegemonic, normative identities. For example, American football promotes very male-centered, heteronormative, nationalistic American culture, yet also exploits race and labor in a way that mimics colonialism (Gems, 2006). Karnatak traditions promote Brahmin-centered music and sounds. In many ways, normative sounds can marginalize non-dominant identities. I will go more into depth with this point further in Chapters 7 and 8.

Sounds are linked to broader scaled/hybrid everyday geopolitics, but some connections exist between and among these identities. Many sounds can also be universal to some identities. For example, when participants described "Tamil" sounds, they referenced the sounds of the tea kettle whistling, curry leaves and mustard seeds popping in a pan full of oil, Tamil TV audible in the background, and in some homes, prayers from prayer rooms or sarees sweeping across the carpet that saturate the atmosphere. They also described that the Tamil language sounded very different than North Indian languages and some even said that it was quite different from other South Indian languages in pitch, tonality, tone, and structure. 
Yet, while there were many commonalities, there were also significant differences in how participants theorized sounds. For example, many sounds that participants used to describe Tamil identities above, other participants used to describe "Indian" and even "American" identities. Specific sounds would reinforce very different identities for each participant. Additionally, I also found that some participants interchanged sound references of Indian and Tamil. Then, they would describe that much of their reference of India was shaped by experiences in Tamil Nadu or having Tamil family. I also found that Tamil participants who had previously lived in places like Delhi, Bhopal, or Mumbai and often made more distinctions based on music, language, or accent. In other words, they had an additional layer of comparison to situate their idea of Tamil. They did not just compare it to the United States but compared it also to the broader notion of being "Indian" in areas where speaking Hindi was a norm.

One sound that almost all participants seemed to emphasize was the sound of traffic - car horns, motors, and the sheer quantity of these sounds. This was used frequently to describe both Tamil identity and Indian identity. Often, it was used interchangeably. While most participants, both first and second generation identified these sounds as either related to Tamil identity or Indian identity, some even suggested these sounds reminded them of their American identities. These traffic sounds also overlapped with identities like Pakistani and Malaysian as well.

In all cases, when discussing sounds of identity, many participants initially connected their identities to particular places like India, Tamil Nadu, or the US. These identities that connected to sound became defined by place. Much literature has been written on the links between identity and place as well as its connection with emotion and 
memory (Keith \& Pile, 2004; Paasi, 2003; Wylie, 2005), but, place was not the only connection to these identities. While place permeated descriptions of identity, it was not the sole factor in how participants conceptualized their identities. In fact, participants relied on a variety of items to discuss sound's role in their identities including place, memories, feelings, other senses, preferences, and experiences.

For example, one participant, Matthew, connected the sounds of engines and cars to a memory that he had growing up in the US. "When I hear a car rev, or it sounds loud growing up I loved fast cars, it brings me back to my previous car. I miss my old car." He associated these sounds with being American but connected them with his memories of his old car, not necessarily the actual place that he grew up. As evident in this chapter, many of these identities overlap and are fluid.

Identities overlap and sentiments regarding sounds can change over time and with experience as well. I asked participants first, if they associated sounds with certain identities that they described, and second, if so, to describe them. For example, Santhya, who lived much of time in the US in New York City also associated American with the sounds of traffic. She said that traffic noises often made her think of taxis in New York City, or large urban areas of the United States. Now, however, she lives in a rural part of Pennsylvania and says that even though she associates those traffic sounds with the US, she is more aware that many parts of the US are quiet. She described that her association of American sounds with traffic noise, now also includes a sense of quiet.

Many participants described the lack of sound in the United States as significant to American identity or loss of Tamil or Indian identity. Many acknowledged that this is true of different places in the world and some connected this to the idea of development. One 
participant, Hamid, who grew up in Madurai, Tamil Nadu with parents from Iran, said that he sees the lack of sound as indicative of a stage of development and also prominence of ecological dangers. Kumaran, who grew up in the US, also hinted at some of the same sentiments, referred to the "sounds of development." He said that the sounds of India reflected a specific stage of development, while lack of those sounds in the US, suggested that it was more developed. "I think the sounds of India reflect the hustle bustle and the population density along with the third world status and growing industrialization mixed in with the old-world things like cows and water buffaloes." Kumaran and Hamid were not the only ones to make this observation. In some ways, these views of sound reinforced Orientalist ideas about developing nations. Many participants though, looked on this sound nostalgically, and others described it as a product of colonization and foreign development.

Unlike many of the other participants though, Hamid was hyper-aware of the differences between soundscapes. He says, like many participants, the issue for him as he is now in the US and identifies somewhat as American, is the lack of sound. Hamid, however, noticed details that others did not. He says,

I'm a wildlife ecologist. I pay a lot of attention to nature sounds. When I do hear birds chirping in America, I kind of like it, but I also know that I'm not in India at the time...In America in a woodlot, there are 10-15 species of birds, maybe less. In India, the sounds are coming from 30-40 species of birds... When I hear sounds of nature in America, it is negative because I feel sad that I'm not in India and can't hear all the number of species. I think, shit, I'd rather be in an Indian forest right now. India has like 11,000 species of birds. America has like 400. 
In this example, Hamid is describing sounds associated with places, but also identities. He described this when discussing sounds associated with being American and being Indian. He describes emotions and connects these experiences to his knowledge and profession. He says the biodiversity in the US in general is not as rich as India and this makes him feel sad in many ways. He associates this sound with being American, with the emotion of sadness, and place. Yet, this place is not specific, but a bit more generalized to include the US as a whole. He brings attention to the fact that the US has depleted so many of its natural species, a product of his experience and knowledge. Because of sound, he feels adamantly aware that he is not in India. From the context of the rest of our interview, to Hamid, being in India is a significant part of being Indian. This is in part, perhaps, because Hamid sees himself as not Indian by visuals or looks, but rather by language, culture, and other aspects of his identity.

Sounds of nature can remind some of being Tamil, while for others, it reminds them of being American. For participants like Bharathi, birds and nature sounds, as she describes, remind her only of Tamil Nadu, triggering her memories. "The natural sound of the environment, it brings my memory back to the time I was raised as a young kid. That natural sound like birds or insects making noises." She said it reminds her of growing up in Tamil Nadu and while she has now lived in the US for almost 40 years, when she hears these sounds, she feels Tamil.

Yet, while these sounds trigger identities of being Indian or Tamil for some, they can also trigger the idea of being American for others. For some participants, these sounds are very clear-cut and distinct. For others, they are hybrid, in-between, and remind them of 
both. It is not my goal to create a list of "Indian" sounds, "Tamil" sounds, and "American" sounds, because I found that more often than not, these sounds overlapped and changed context based on the experiences of a particular individual. But for some participants, they were distinct. For those like Kumaran, nature sounds remind him of growing up in a rural part of Ohio. He described that they reinforced his American identity, rather than his Tamil identity. Indian and Tamil, for Kumaran, is a place with a lot of people, urban, industrializing, street noises, music, chanting, etc. While the US he says:

I associate rustling leaves and swaying branches, chirping of birds, the sound and popping noise of wood burning in the fireplace in the winter which wouldn't be needed in a warmer place like India, the more organized sound of highway or street traffic, and the sound of crowd noise at baseball and football games.

For many participants, the sound of sports and games were very "American" sounds. But, these could also be hybrid and/or "Indian" sounds if associated with sports like cricket. Some participants said that sports sounds reminded them of being both Indian and American - they often blurred these sounds when thinking about identities. Likewise, participants like Prisha said that sounds of a food market reminded her of both American and Indian identities simultaneously, especially because she lived in an area in the US where food markets were available.

Other participants, for example, associated fireworks with both Indian and American identities. Many associated them with American identity, reminding them of the $4^{\text {th }}$ of July. These included both first and second generation participants. For others, 
however, fireworks reminded them of celebrating Deepavali in India, not the United States. In other words, many of the same sounds triggered a variety of memories and associations with different identities - some distinct and others hybrid. Generational differences were not as much a factor as the specific life experiences of the individual. I did find, that the more time participants had spent in the US, the more likely they were to associate fireworks with the $4^{\text {th }}$ of July. However, even so, this was not the case with all participants and some participants, who had been in the US even for a short time, mentioned fireworks and $4^{\text {th }}$ of July. Similarly, those that had spent a long time in the US still associated fireworks with being in India or Tamil Nadu during Deepavali.

But, despite the many differences in sound associations, participants did agree on some sounds. Many participants agreed that the level of noise in India and/or Tamil Nadu was much higher when compared to the US. Though, each participant reacted differently. Hamid described both positive and negative emotions somewhat contradictory when discussing the noise level. For nature, he described this as positive. However, for other environments, he described this as negative. He referred to this volume of sound as 'noise pollution,' and specifically ties it to Tamil Nadu. While he describes sadness about living in the US as an American with depleted resources, he also describes the frustration of his memories of being Tamil in Tamil Nadu. He says that all the loud speakers, the politicians, religious sounds, including Hindu and Christian, make him angry.

This time..., it has become so bad, the church had Tamil songs blasting, and the temple around the corner, the church had decided to blast Tamil religious songs for like two hours. I'm like who the hell gave you the right to do that? 
Quite a few participants described anger at 'noise pollution' in their memories of Tamil Nadu. Yet, they did not always associate the noise pollution with the same things. Ruth, for example, also describes anger and irritation, but instead describes it regarding cars and beeping specifically. Yet, she says that later, when she moved to the US, she felt sad when she did not hear them. Many participants responded this way and often contradicted their previous statements. I asked her how she felt now, and she says that she feels peaceful, not having to hear them, but still sometimes feels sad. That is, until she goes to India and then she is no longer sad, but frustrated and angry. But, upon return, she again might feel sad. Many participants described this contradictory feeling regarding noise levels. Others, while they described these sounds, did not feel strongly about them and just associated them with memories of Tamil Nadu or India. Often, participants described that the sound of car horns reminded them of a memory in Tamil Nadu or India.

\section{Food and environmental sounds}

I found that many participants also described connections to identity through sounds of food and cooking. In fact, most participants referred to connections to food that often then connected to their families. If they did not describe specific memories connected to food, they often referenced food at some point in the interview. Almost every participant connected sound to food and smell at least once during the interview. I did not specifically ask questions about food or smell, but almost everyone addressed them in some way. The sounds of boiling tea and cooking food were two sounds almost always mentioned. Although participants described cooking of different items, they always described the 
sound of oil and spices popping in a pan or the sound of the whistle from boiling tea. Many went further to describe the sound of tea pouring in steel cups back and forth. Some described the sound of the pressure cooker, but also described the oil popping in the pan.

Food overall was important to scalar distinction of Tamil for almost every participant. Though, the types of food described varied depending on if the participant was vegetarian, Brahmin, Hindu, Muslim, or Christian. Regardless of preferences of veg or nonveg, many participants described items like idli, sambar, dosai, vadai, rasam, and a few other staples of South Indian food. While there were distinctions with regional food and participants mentioned that food varies based on family background and recipes, many described that food was a universal part of identifying as Tamil. For example, Paranthakan says that "every person will make a different sambar. Everyone gets tired of their own sambar and wants to try someone else's." Paranthakan and others described that North Indians do not make sambar the same as Tamils. Sambar, while its recipe can vary, has a very specific 'South Indian' taste. Sakhti describes an experience where she ate Gujarati sambar.

One of my Gujarati friends, she bought a house and was having a housewarming puja at her house... One of the foods there was sambar and I didn't recognize any of the other food. I got myself a big bowl of rice and sambar. I didn't know Gujarati people added sugar to their sambar. I felt obligated to eat it all. I want to cry so much but didn't want to insult my friend. I'm like, never again am I going to try anything other than Tamil sambar. Tamil food is the best. 
Many people identified as Tamil through food, distinguishing Tamil from other regions of India. Nazeem, for example, identified herself as Tamil because of the food that her family ate. She is a second-generation Pakistani and Indian and separates her scalar identities by food. She said that she was Indian because she had "a very specific requirement for biryani", describing that it needs to mimic a local, Hyderabad-style biryani. Though, overall, she describes herself as South Asian, she references the local importance of Hyderabad ${ }^{22}$ to her South Indian identity when describing food. She attributes the region to her specific food identity. She said she was "Madrasi"23 because she liked idli, sambar, dosai, vadai, and other Tamil foods. She changed her scale of reference when discussing her identity as it related to food. She often was reminded of these foods when she heard sounds of the kitchen. Cooking sounds brought back memories of these foods and specific memories of her family cooking in the kitchen.

Almost every participant who described the sounds of food, associated these sounds with a particular memory of family members in the kitchen. Often, these were mothers or aunties. Very few participants mentioned memories of fathers or uncles. While, it seems that these values of cooking and food were often gendered, and this is an important observation, this chapter focuses more on the hybrid and scalar identities regarding Indian, Tamil, American, and others. ${ }^{24}$ One participant, Sakhti, described the sounds of the mortar and pestle and the sound of mashing up ginger and garlic.

\footnotetext{
22 Hyderabad is located in the South Indian state of Telangana

23 This term will be discussed further in Chapter 8.

24 It is important to also not that while for many, memories consisted of mothers or aunties cooking, for those born in the US, cooking was not based on gender identity. Both female and maleidentifying participants equally described cooking or not cooking at all.24
} 
I have one of those - a really old one from my dad's great aunt. I don't use it for cooking, but sometimes I like to play with it and make noises with it - like dad's great aunt used this. It makes me think of her. I met her only once after we moved to the US. She was really old when I was young, and she was bed-ridden, so I only have that one memory of her.

Sakthi described this in association with being Tamil. She does not actively refer to it in her daily experiences but thinks about it from time to time. She considers this sound and its connection to these memories as part of her identity as Tamil. Many participants connected these memories and specific sounds of food to their identities as Tamil.

Another example is provided by Vimala, who described her connections and memories with family through the sounds of the street. "Whenever I hear lots of tracking and beeping, it reminds me of street food. We'd get paratha on the street. I have many memories of sitting on the floor and eating the paratha with my uncle on a banana leaf." She reflects on these memories fondly. Vimala grew up in the US but thinks often about the time that she spent with family in India. Hearing sounds of traffic in the US, brings back these memories of food and being Tamil. Many participants connected sounds of food to memories or experiences of being in Tamil Nadu, India, with Tamil family, or specific Tamil influences in their lives.

Though memories of India and Tamil Nadu were significant, food sounds were also not always connected to these places. Some participants described these as connected to their American identity. One participant mentioned found that the sounds of the market, like the West Side Market in Cleveland, reminded them of being in the US, but also getting 
fresh vegetables and fruits in India. In this case, the participant described a hybrid identity, because she associated these sounds with being American and being Tamil. She said these sounds overlapped and reminded her of both identities, which sometimes blurred. Many described the experience of sounds overlapping with identities, especially in regard to food. Participants might recall street food vendors and think of India. Yet, they would simultaneously think of food cooking at local American festival food trucks at fairs or carnivals. Sometimes, participants would either think of US festivals or Indian or Tamil festivals yet would describe the same sounds and similar experiences with different reference points. For some, it was American, for many it was Indian or Tamil, and for others, it was many blurred identities.

Many participants also mentioned smell. Some described smell as being more important to memories than sound, but often that all these senses were interconnected. Smell reminded them of food, or reminded them of the process of cooking food, and thus triggered memories of sounds of cooking. One participant even mentioned the smell of Indian grocery stores in the US triggered her memories of grocery stores or buying food in India. While I am highlighting the importance of sound, I recognize that senses are very interconnected, as many participants described - much like how various identities are connected. Even at participant observation events, the sounds and smells of cooking or food were quite connected.

Sounds like music, language, or accent were not often connected to smell, but for environmental sounds, smell was quite important. Often, when describing environmental sounds like traffic or nature people would describe the smell of traffic and pollution or the smell of nature. For nature, Punniya, who came to the US from Chennai in 2004, describes 
the "natural" smell of rain connected to her Tamil identity. "Before the rain starts, some kind of smell comes. I love that smell. I don't know to explain it, but it is very rare that we smell that here (in the US)." While Punniya did not associate the smell of rain with the US or being American, others described the smell of the rain as integral to their experiences as an American in the rural US. Again, smells, like sounds, also depended on the individual experience and context.

Environmental sounds, especially, more than music, accent, or language, were connected to smell. Food and memory strongly shaped participant responses related to sound. Food was also present in every Tamil and Indian event I attended. Even in many of the Northeast Ohio Tamil Sangam events described, food and memory, coupled with environmental sounds saturated the space. In NEOTS events, a variety of Tamil dishes, that change at each function for variety, are served before the events, plays, or performances and the smell overpowers the event. Food was a significant part of environmental sounds. The sounds of cooking particular foods were intertwined with smells, memories, and a multi-sensory experience. Though this study does not directly focus on food, its connections to identity are quite significant. Overall, environmental sounds were significant to defining identities at events and through Sangams, but also through individual participant experiences.

\section{Music and identity}

Participants sing the state song of Tamil Nadu in a local high school in Northeast Ohio Cleveland suburb during the cold months of November. Soon after, the voices shift to a lower tone, and participants sing in unison the Star-Spangled Banner. Then, just as fluidly, 
announcements in Tamil ring out over the microphone, as the Deepavali, the festival of lights (known as Diwali in other parts of India) continues with hours of musical performances, arranged by the Northeast Ohio Tamil Sangam. As the audience prepares to depart, the Indian National Anthem concludes the program. A few months later in the cold, snowy month of January, this repeats as the Harvest Festival of Pongal is celebrated and few months after that, the Tamil New Year, Chithirai Thiruvizhia.

In late March and early April often within weeks of Chitirai Thiruvizhia, the Waetjen Auditorium of Cleveland State University in Cleveland, Ohio, Tamil-language conversations echo as music of the pancharathna krithis - the beloved South Indian music composer Thyagaraja's five greatest compositions -flood through the hallway. The mridangam, ghatam, kanjira, Saraswati veena, violin, and other South Indian or Karnatak instruments hum while singers from Chennai, India bend their voices to complement the music. Many of Thyagaraja's compositions are written in Sanskrit or Telugu, but Tamil rebounds from the chattering audience. The culmination of sounds reverberates in the hall for a central performance of Karnatak musical heritage, that many Tamils associate with Tamil identity. ${ }^{25}$

Music is also very much connected to memory and emotion. Many scholars that engage with NRTs and beyond have looked into music and its emotional effects on listeners and/or bodies (Anderson et al., 2005; Gilroy, 1993; Jazeel, 2005; Saldanha, 2007; S. Sharma, 2006; Smith, 1994; 2000). Smith (2000) and Revill (2016) acknowledge music's importance socially and politically to identities and geography. Music is a major art form that "inform(s) geographical interpretations of the cultural landscape" (Smith 1994, p. 235)

25 I will discuss the complexities of this association with identity further in Chapter 8. 
while "musical aesthetics" differ between regions and 'cultures', creating a collective difference in these aesthetic preferences (Jazeel 2005).

But while music is a collective experience, it is also very much individual. Sounds that evoke emotion or memories for one person may not have the same effect on another (Jazeel 2005). Music can also bring memories of the past into the present moment, connecting people to specific periods in their personal life experience (Anderson 2004). Music is referred to as universal, yet it is also very much individual (Jazeel, 2005). Music can relate people collectively yet is grounded in individual experience and interpretation (Jazeel, 2005).

In events and performances, music was important to distinguishing the regional importance of Tamil identity from other parts of India. This surfaced through political songs like the state song of Tamil Nadu, or through popular songs based out of Tamil Nadu's movie industry, Kollywood. If more popular Hindi songs were present in events, the purposeful change of the lyrics to Tamil was evident. For example, any and all songs originally recorded in Hindi were played in Tamil throughout events. Aside from the national anthem of India, Hindi language songs did not surface in any events. This was true for Tamil Sangam events as well as for the Cleveland Thyagaraja Aradhana.

Music was also emphasized through its historic connection to Tamil Nadu through literature, architecture, and landscape. This connection was not just reinforced in events but was also referenced by many participants. Some participants referenced the links to music and temple architecture. Rittika, for example, sent me multiple articles and websites with information of many Tamil Nadu temples that have been influenced by music in design and structure. She described how this was unique and very important to Tamil 
Nadu's history. Rittika said that this incorporation of sound is crucial to her identity, and especially to being Tamil.

Music was also linked to landscape and literature. Previously, I outlined the concepts of tinai as well as the importance of the literature from the Sangam Age. Much of this referenced literature connected directly to music in multiple ways. For example, references to early texts such as the Silapaddikaram that describe many musical scenes or references to the origins of Karnatak music. Often highlighted at events like the Cleveland Thyagaraja Aradhana or by participants in interviews, is that Karnatak music emerged in the Kaveri Delta in Tamil Nadu, making it significant to some interpretations of Tamil identity (Viswanathan and Allen, 2004). Though, as I will discuss in Chapter 8, many did not share the view that Karnatak music was significant to Tamil identity, but rather reinforced an upper-class, Brahmin view of Tamil. Nevertheless, regardless of caste or class, there was a consensus that Karnatak music did have connections to Tamil Nadu and Tamil identity, whether those were positive or negative.

Beyond classical music, popular and folk songs were also referenced by participants. Classic artists like Ilayaraaja, were highlighted as important to participants. In performances and event, artists like these were also highlighted. Many filmi songs in the movie industry of the South challenge dominant views and visuals established in Bollywood (Ravi, 2008). Live music performances at specific places and times create "a sense of community identity" that resists homogenization into larger identities (Smith 1994, p. 236). Thus, these can be enacted regional scales that emerge for the purpose of not just performing identity, but also resisting homogenizing, dominant identities. So, in Tamil 
events and functions, the regional lens of Tamil Nadu was often highlighted through preference for state political songs and popular film songs in Tamil.

In the events, Hindi was never used nor referenced, except at the end of the functions to reify that the event was Indian Tamil, not Sri Lankan. Sri Lanka was never directly referenced, but the idea of India and Tamil being Tamil Indian was subtly reinforced throughout all events and performances. As mentioned in Chapter 3, Sri Lankan Tamil associations are often separate from Indian Tamil. Though, sometimes, participants from Sri Lanka will participant in Tamil Sangam events in Cleveland. Again, these categories and identities overlap or differ depending on situation or context. But nonetheless, these events were defined as Indian Tamil through music, language, direct statements, visual items like dress or flags, and even food. Music, especially, was first used highlight the regional identity of Tamil and subsequently, the national identity of Indian.

Thus, musical sounds can be central to performances of Tamil identity in the United States and more specifically, to highlight enacted regional scales that resist threats of homogenization. Music was intricately weaved into many performances, events, and everyday lives of Indian Tamils and many, if not most maintained some emotional connection to music. Music was not just symbolic for Tamil identity, but it also served as a marker for other identities as well. It was a purposeful scalar mobilization, as demonstrated in these events, and also demonstrated hybridity, blurring identities.

For example, for some participants, music symbolized overlap and blurring between Tamil identity and Southern US identities or even urban American identities. What were described as universal forms of music like 'hip hop' or 'rap' some participants mentioned they saw blurring in both Tamil and American music. Other participants 
compartmentalized music. Matthew, for example, thought of Bollywood music when discussing Indian identity, but thought of country music, which he loves, as part of his American identity. Nazeem, thought more about hip hop, but also thought about country or pop music as related to American identity. Though, Nazeem mentioned, that although sound was significant to her identity (rating it a 5 out of 5), she thought of music as more of an environmental sound, rather than an emotional connection.

Nazeem said she did not really like music. Maari, on the other hand, thought of himself as a universal music lover. He did not always distinguish between scalar identities when referring to music, describing more of a blurred, hybrid experience. He mentioned that music is global because in many cases, local music often influences other music around world, connecting these types of music. He described how the influence of 90s grunge/rock bands in the US can be seen in some of the new scenes of Tamil or South Indian music.

Music can "disrupt ideas of purity and origins" as (Sharma, 2006, 318) shows through his case study of British and Asian fusion music. Bhangra music embodies hybridity in the Punjabi British diaspora that fuses traditional folk sounds with some Western elements. Sharma (2006, p. 324) suggests that the music goes beyond the "mainstream" to become an important part of "a lived diasporic identity". Music is a way, according to Sharma (2006, p. 324), to affirm an "Asian identity" and "agency" while also reacting to multiple layers and levels of racism. He suggests that music can be hybrid, and "defies national boundaries and cultural authenticities" (Sharma, 2006, p. 325) to be a source of resistance to homogenizing Eurocentricity.

While Maari did not directly express these sentiments, his understanding of music echoed much of what Sharma (2006) suggests. Though, for Maari, he described himself as a 
universal music lover, yet still compartmentalized music into various identities. His experiences with music were both hybrid and scaled. They were scaled in moments when, for example, he mentioned that he enjoyed Karnatak music and it reminded him of being home in Trichy. They were hybrid in moments, for example, when he described that some of the best rock bands around were from Tamil Nadu but blurred with many 90s rock band styles. These bands were not like the traditional Tamil artist Ilayaraaja, that other participants referenced. ${ }^{26}$ They were rock bands that he compared to Blink 182 or Incubus. I listened to many of the songs he sent me. For some bands, the lyrics were in English, and they did sound like Blink 182 or another American band from the 90s. Even bands with Tamil lyrics, was stylistically similar to a 90s US band. These were examples of a blending of music. They were not traditional Tamil music that many participants described, but they were not American either. They fit what Sharma (2006) describes as "defying" authenticity or categories, making them quite hybrid. Maari described this type of music as important to his overall identity of being a music lover. He described this as neither Indian, Tamil, or American, but instead a more universal, connected identity that defies these categories. Music is a way to assert or remember specific parts of identity, yet also remains hybrid and non-binary. For example, Maari describes that music is global because it is influenced by local. Local and global are interconnected. While Maari may not have described this as a hybrid process, his interpretation of global music described the conditions of hybridity and in-betweenness. It also reflected Antonsich's (2018) findings that the local and global are hybrid and in-between, not necessarily in opposition. Music,

26 Though, it is worth noting that Ilayaraaja also hybridized music, drawing from classical Western music, Tamil folk music, and other sources. 
for many participants, at times, traversed multiple identities. In many ways, it was hybrid and in-between. Music for some, was a personal journey that became a hybrid experience, bridging multiple identities and even senses. Sam, for example, came to the US in the 1960s start a music career and eventually became a photographer. He initially pursued sound and through a variety of circumstances, found his way to visuals. He says that he has always seen these senses as connected.

Sam's love of music led him to become a world-renown photographer for the United Nations. Sam describes himself as "global citizen beyond borders". Yet, also acknowledges that he is "probably Tamil". When asked to describe Tamil, he referenced Tamil literature, food, attitude, dance, and music. Chennai, Tamil Nadu is where he spent much of his early life. Yet, while he was in Chennai, he describes that "I love blues and even when I was young growing up in Chennai, I was exposed to American blues music. One of the reasons I came to America was to be a folk singer." Sam even had a talent for yodeling and was featured on TV show in the 1970s showcasing his yodeling talents. He sent me a video of this clip as he was describing his connections to music.

Music, in general, Sam describes as a "universal language." Sam also describes that he loves the sound of the ghatam, ganjeera, and "all the south Indian percussions." He loves to listen to Konakol which he describes as "our scat singing". He even connects his overlapping, blurred experience with sound and visuals to his career. He describes a time when "our famous singer MS Subbulakshmi came to NY" and he spent the day photographing her for an album. This was a day where he merged his love of sound and visuals. Photography became "a way of life" for him. Yet, simultaneously, he is also tied to the aural. Music was a significant part of his life experience and was intricately weaved into 
his identities. He referred to music as South Indian, at times, and others as Tamil and Indian. Simultaneously, he describes his experiences with music as also American, compartmentalizing the influence of blues and folk music.

Folk music, though, bridges many scalar identities - Indian, South Indian, Tamil, and American. In many ways, folk music became a symbolic bridge between these various identities that blurred. Folk music was not just symbolic of American or Indian or Tamil music, it was connected to all of these identities in a very hybrid way. It was folk music from various localities in the US, India, and other parts of the world that influenced a larger, global connection between his identities. These identities were scaled and also hybrid. While environmental sounds and food seemed to bring out scaled identities, music demonstrated how these scaled identities were hybrid, connected, and even in-between. Almost all participants referenced a variety of music relating to both scaled and hybrid identities. They also related music to feelings, moods, and some even described music as reaching an intangible part of their identities that could not be described. Each participant's responses and discussion of music varied. While music demonstrated hybridity, it also reinforced hierarchical scale. For example, many participants thought of Bollywood and Hindi music as connected to a broader, Indian identity. Though, some who described this connection mentioned that they personally did not feel connected to that music or language and that is when they identified regionally as Tamil.

But connecting to Tamil through music was complex because it disrupted the idea of a universal Tamil. Music was not universal for all participants and no one had the same definition of "Tamil" music. Even with the state song of Tamil Nadu, those born in the US who never attended Tamil school did not know this song, nor conceptualized it as part of 
their identities. Yet, in official events and performances, it was a staple to opening all events. Many participants did not know or could not remember the state song of Tamil Nadu, but yet to Tamil Sangams, these was the first song, often emphasized as the most important.

Another significant barrier to a universal Tamil identity was the connection of Tamil identity to Karnatak music. Many suggested that Karnatak music was connected to Tamil identity, but some were adamant that it should not represent Tamil identity. At events and performances, Karnatak music was often utilized as backdrop or even for dance performances of Bharatanatyam. In fact, at festivals like the CTA, Karnatak music was mobilized to demonstrate the differences between North and South India. South India, in this event, was often tied to Tamil identity and to Karnatak music's origins in the Kaveri Delta in Tamil Nadu. But more importantly, Karnatak music signified South India while Hindustani, a branch of North Indian classical music, signified North India. For artists and event leaders, Karnatak music was pure. It resisted the influence of any invaders or cultures that permeated the North. Additionally, many stressed that Karnatak music was much older than Hindustani and its roots were much deeper. A map of the divide between Hindustani and Karnatak music was printed in the 2015 programs. Since then, it has been removed. In fact, in the last program, a few Hindustani music concerts were showcased.

Karnatak music did not just demonstrate a scalar link to Tamil and South Indian identity. It also described a new, hybrid identity for American Tamils living in the United States, especially the second generation. These identities were described and represented as blurred and in-between Tamil and American. Simultaneously, they emphasized the blurring between local origins and global influence. In this way, global, local, national, and 
regional co-existed, creating hybrid, blurred identities. During the opening performances of the 2016 and 2017 festivals, emphasis was placed on the next generation of IndianAmericans to preserve the heritage of Karnatak music. Regional, South Indian music was to be preserved by Americans in the United States, and more specifically, in the locale of Cleveland, $\mathrm{OH}$. One famous musician introducing the 2016 festival, who last came to the US for CTA 43 years ago, V.V Subrahmanyan, described that "North America will continue the legacy of divine and God-inspired Karnatak music." North America, and more specifically, Cleveland, $\mathrm{OH}$ was a bridge, a hybrid space, that created a new chapter of this South Indian, Tamil identity.

The cover of the 2016 CTA official program guide reified the message of hybrid, inbetween identities in Cleveland, OH. It referred to Cleveland as "America's home of Carnatic [sic] Music." The new map inscribes a heart around Thyagaraja highlighting Cleveland, Ohio as the center location emphasizing travel from East, West, and North, presumably Canada, India, and perhaps parts of Southeast Asia (Figure 2). In essence, it demonstrated that all Karnatak musical roads lead to this in-between, hybrid space of Cleveland. This was more than just a space and place, but also an identity in need of preserving and maintaining, as emphasized throughout the program. 


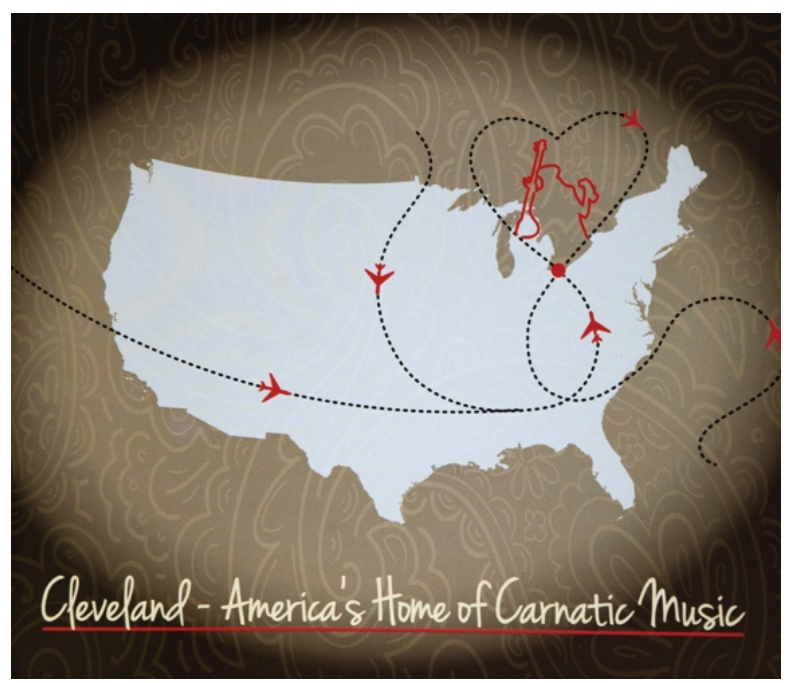

Figure 6: 2016 Cleveland Thyagaraja program guide with map of Cleveland, OH at the center. Source: 2016 Cleveland Thyagaraja Aradhana Program guide.

Tamil identity was hybrid, blurring local, global, regional, and national identities. It was South Indian, American, and even Cleveland, but certainly not homogenous. Karnatak music was mobilized to bridge Tamil, South Indian, and American identities in a hybrid and interconnected culmination in the Cleveland Thyagaraja Aradhana. In recent events, Karnatak music has shifted from being Tamil identity in the US, to now representing the US, and even more specific scales like Cleveland, $\mathrm{OH}$. The locality of Cleveland is purposefully mobilized to define the future identity of Karnatak music. It was hybrid, but also emphasized traditional scalar identities.

In interviews, many participants said that Karnatak music was part of Tamil identity. Many were proud that Karnatak music is Tamil, but also American. Others were proud of Karnatak heritage and connections to Tamil history. Others, just referred to it to describe Tamil contributions to the art world. But, while some said that Karnatak music was a staple of Tamil identity and others, suggested that it was offensive to represent it as Tamil identity. While scales like Tamil or South Indian were mobilized against North India, 
Bollywood, and Hindustani music, Tamil scales began to break down as well. Local scale became more important that regional scale of Tamil because many critiqued Tamil for homogeneity and reifying upper class narratives.

In December of 2016, I traveled to India for the December Music Season for a second time (the first time was in 2013 as I was working on my Master's Thesis) to watch as performers and audience members from the US were involved in many of the Karnatak music performances. In the US, the local connection to Chennai is emphasized at the Cleveland Thyagaraja Aradhana, where many people describe that its traditions are important to pass onto the second generation. Yet, in Chennai, I had about 15 conversational interviews with non-Brahmins who described the festival as very "Brahmin" and upper class. These conversations ranged from University professors to Auto and Uber drivers. To them, Karnatak music had nothing to do with Tamil Nadu. It was a Sanskritized ${ }^{27}$ version of music that claimed to represent Tamil.

One of my expert interviews, an individual who specializes in music in Tamil Nadu, suggested that Karnatak music is part of larger caste politics.

So, in Tamil Nadu, sound is a caste marker. The kind of sounds you use formally and informally distinguish you as a person who belongs to a particular caste. You have the right to that particular sound, so others cannot use that particular sound. So the sounds (referring to Karnatak music) ....only belong to the Brahmins. These sounds

27 This was a term used by a few different individuals that I interviewed. Sanskrit in Tamil Nadu is only accessible to upper class or Brahmin families. Most people do not understand Sanskrit. 
once upon a time belong to Devadasis ${ }^{28}$, then they were classified as - from that community Brahmins appropriated all these sounds.

In my interviews in the US, people were not as direct about Karnatak music but did suggest that it only represented a specific group of Tamils. Some individuals described that Karnatak music always took the spotlight, rendering local folk music traditions invisible in broader representations of Tamil, especially in the US. One individual, who used to live in the US, but has since relocated to Tamil Nadu said, "See Thyagaraja Festival not every Tamil will go. The Brahmin groups only will go.... These are the groups that understand the music. Wheras none of us can understand Karnatak music. The base of that music is Sanskrit." In other words, he suggests that people in events like the CTA represent Tamil identity through Karnatak music, which ignores local village folk traditions and many people who are not Brahmin or upper class. Sanskrit also comes from the North and is very different from Southern Dravidian languages. In some ways it is a scalar problem that is connected to caste politics. It is homogenizes and ignores local, non-Brahmin traditions, reifying upper class, Brahmin, urban narratives in places like Chennai. It also in some ways highlights subnational scales like North India over South India. I elaborate on these issues more in Chapter 8.

Music had many implications for scalar and hybrid identities but was important to many participant's experiences. It was symbolic of complex identity, which I demonstrated was scalar, hybrid, or both. In events and performances, music was used to highlight and reinforce particular identities and, in some cases, purposefully mobilize regional identities

\footnotetext{
${ }^{28}$ Servants of the temples, often lower caste.
} 
against national identities as in the case of Tamil and Indian. It also mobilized regional identities against other regional identities as with South Indian and North Indian. It reinforced the importance of the local, like with Cleveland, $\mathrm{OH}$, or erased the importance of the local, as with Karnatak and folk music. Simultaneously, it was also hybrid - blurring local and global identities. Music that represented the larger "India" was also used to define Tamil as nationally Indian rather than Sri Lankan. Yet, Tamil identity was represented in many circumstances by Karnatak music that many argued was symbolic of a particular caste - Brahmin and silenced other definitions of Tamil.

\section{Language}

Most participants, when asked about language, focused on Tamil. Some mentioned Hindi as the national language of India and described its importance to maintaining unity, but the majority of participants focused on Tamil. For example, the tensions between Lakshmi and Arvind centered on identifying as Tamil or Indian, but often referenced language - like Tamil or Hindi. It was Lakshmi's father's fight for Hindi and unity of India that inspired her to identify as Indian before Tamil. It was also partly Arvind's family's experience with speaking Tamil, that made them feel different than North Indians. Nonetheless, when it came to language, most participants chose to discuss Tamil more than any other language. Many said that it was special and unique, different from other languages.

Linguistic and language geographies have examined language quite robustly, especially through semiotics (Dewsbury, 2010). Yet, NRT scholars like Dewsbury (2010) stress the importance of moving away from semiotics to examine language as a raw 
experience. Dewsbury (2010) advocates for understanding language and words as an "experience and event", rather than focusing on their inherent meaning alone. To demonstrate, he describes the difference between doing art and a work of art - one is the act, the other is a representation of a moment. He suggests that language can be looked at as a representation, but it also must be examined as an event within a moment - that is to say that we experience language in tones, timbre, tonality, inflection, pitch, texture, etc. Kanngieser (2012, p. 339) stresses thinking about language as "sonic phenomena" rather than purely "linguistic phenomena subsidiary to speech." In other words, she advocates for examining the way that language sounds, not just focusing on what languages means.

For instance, people often described Tamil as a beautiful language, giving an emotional response in a moment that reflected a feeling of beauty. As mentioned, throughout events and performances, many songs were changed from Hindi to Tamil. In this case, people at the events have mentioned that it is not the meaning of the song, but the language and sound of the language that was important. The sound of Tamil is "mighty" or "ancient" as some have characterized it. This evoked feeling and emotion of awe and even pride at the sounds of Tamil.

For most participants, there was an incredible emotional connection to Tamil. Many described that when they heard Tamil, they felt an immediate sense of comfort, even if they were not fluent in it. There was something familiar about the sounds of the language that created a sense of peace for many or brought back memories of family. Jaya mentioned, "When you talk about Indian sounds, when I hear people speaking Hindi or some other language that is not Tamil, I don't feel a connection to it. It is interesting, when I was 
growing up there were fewer Tamil people. I hear people speaking Tamil at the airport, my ears perk up and I definitely notice it and I get drawn back to a Tamil identity."

For those not as fluent in Tamil, they mentioned that the sound of Tamil reminded them of their parents, grandparents, family members, or memory they had when they were younger. For those more fluent in Tamil, they also described a sense of familiarity or feelings of peace or happiness when they heard Tamil spoken, because it is not common to hear in the US. Maari describes it, "Tamil is a very intricate language which 200 plus characters. There is a phonetics for every sound we can make. It is a beautiful language - it makes you feel good. There are words that cannot be said in English."

Other participants, like Rittika, described specific sounds that are unique only to Tamil. "You know the special zh in tamil - that is a special sound that is not in the Northern languages and not in Sanskrit or Hindi." Rittika was not the only one to mention this sound. ${ }^{29}$ Many participants described this as being uniquely Tamil. The "zh" in Tami(zh) is produced when reaching the tongue far back in the throat almost touching the palate, but not quite. It is known as a retroflex consonant, and one that is very difficult for non-native Tamil speakers. In TMM, this is the hardest consonant for students to pronounce.

Participants varied in how they described Tamil, often based on level of fluency. For Durga, the lack of Tamil became part of her identity. She can recognize Tamil, but because she is not fluent, it serves as a placeholder for an identity that is connected to language, but she does not have the ability to access. Other participants, like Vikraman, described that Tamil, for him, was "the sound of people speaking it." The way that it sounded in the mouth was different than North Indian languages. Matthew, like Vikraman, describes that it

29 Though, this sound is not unique to Tamil and appears in other South Indian languages. 
sounds different than other languages, even other South Indian languages. "My wife always teases me about my Tamil language - she said it sounds crass compared to Malayalam."

Matthew also says that he struggles with the different versions of Tamil, the ones that he describes as "poetic". He says they not only sound different, but he is completely lost when listening to "poetic" Tamil. In the Northeast Ohio Tamil school, this was addressed. Instructors often addressed differences between classical, formal Tamil and colloquial Tamil. Especially when reciting the Aatachudi, several translations ensued. First, the lines were translated to colloquial Tamil and then translated to English. Tamil, as explained, has several layers and sounds. On TV programs and news stations, Tamil is much more formal, and many described, even those who grew up in Tamil Nadu, that they don't always understand it. Yet, even this Tamil is not the level that one might see in poetry or prose. The sounds and way that Tamil is spoken changes depending on context. Many, often, were familiar only with colloquial Tamil.

Even if participants did not speak Tamil fluently, understanding the differences between North and South Indian languages was significant to identifying as Tamil. Many participants, if not all, were aware that there were significant differences in the way that North and South Indian languages sounded. Additionally, at Tamil schools and many events, often in passing, people would mention significant history of the Tamil language and how it was different from other languages in India. Regional and scalar distinction of the Tamil language and the rest of India was emphasized and underscored through events, performances, and within interviews.

Many linguists point out differences between North and South Indian languages as well. While Hindi, Bengali, and many of the Northern languages are based on the Indo- 
Aryan language family, the Southern languages, the oldest recorded languages on the subcontinent, come from the Dravidian family and have their own characters, script, and literature and thus are unique sounds in the broader Indian soundscape (Ramaswamy, 1997; Wolf \& Sherinian, 2000). In other words, Indo-Aryan and Dravidian, more specifically Hindi and Tamil, even though they are both languages within India, sound very different from one another. Depending upon the listener's native language, one language sounds like it is coming from the chest, while the other sounds heavy in the mouth. After independence, Hindi became one of the national languages and its soundscapes now dominate representations of India, especially in Bollywood (Devadas and Velayutham 2008). However, in Tamil Nadu Hindi is not often spoken or emphasized (Schwartzberg \& Bajpai, 1992).

Some participants even mentioned that Tamils living in the US, change the structure of their sentences to reflect English. So, in essence, many said that this changed the way that Tamil sounded. The meanings were the same, but the sound and how it flowed from the mouth sounded like English, not Tamil. Some described that they unintentionally changed the way they spoke Tamil after living in the US after so many years. However, it was not just the sentence structure that changed, but the way that the voice was moderated. They described that this also changed the perception of their speech as well.

Kanngiser (2012) builds on this explaining that geography has looked heavily into music and sound, but not necessarily into how people "listen". In other words, voices, speeches, and the way people talk have meaning. In Chapter 2, I mentioned how she shows that Obama's perceived speech tones as "black" or "white" were heavily criticized during his campaign. Additionally, she explains how higher-pitched, softer voices are thought of as 
feminine, while lower pitched, louder voices are thought of as masculine or how the speed of speech changes. The loudness of a voice can also determine power, while silence can be a sign of protest. She posits that " $(t)$ he inflections and modulations of the voice contain forces that we must become more conscious of" (Kanngiser, 2012, p. 348).

Decibels, for some, could determine the way that they are perceived by others. Pandian, for example, compared the loudness of Tamil with the softness of English "I don't know if this is true, but I think if conducted research we would find that different languages have different average decibels when people speak those languages. I, for myself, have come to realize that English is one of the softest languages that is spoken around the world and Tamil might be one of the loudest languages around the world. Because people who speak Tamil tend to be more loud, so the sound kind of gives certain characters to the person it comes from."

Many participants described the loudness of Tamil. In fact, almost all participants described Tamil as loud. Arvind, who studied sound in a quantitative way for his research, said that if one was to look at all the frequencies of the vocal chords, a Tamilian's voice would show very different patterns than someone speaking native English. He also mentioned that Tamil is "unmoderated." He said that English emphasizes and deemphasizes, while Tamil does not go "up and down." Pandian, however, described that Tamils place emphasize on everything, unlike when people speak English. This emphasis, he says, is a characteristic of Tamil identity.

Not all participants agreed that a characteristic of being Tamil was being loud. Fathima, for example, grew up in the US with what she describes as a quiet family. She said she realized this when she was introduced to her husband's Lebanese culture. "The decibel 
level is a lot lower (in Tamil). My husband's family was like screaming at each other. I feel like I always grew up in a much quieter environment. My husband's culture is so loud." Fathima describes being Tamil as being quiet, but many others describe it as very loud and outspoken. Simply put, for many participants loudness/softness of language was important to Tamil identity.

\section{Language at events}

At Tamil Sangam events in the Cleveland area, the entire program is conducted in Tamil. Tamil leads conversation and official events. The event facilitators speak only in Tamil and if he or she calls anyone on stage to speak, they also speak only in Tamil, occasionally using a few English words within their sentences. Beyond official programs and conversations, Tamil music, often in Tamil, is also integral to events. As mentioned in the previous section, most of the events of the Tamil Sangam open with the Tamil state song of Tamil Nadu (the invocation to the Tamil Mother, Neerarum Kadaludutha). Throughout the events, Tamil language songs are performed, acted out, or sung either on stage or by audience members and many Hindi-language Bollywood hit songs are changed to Tamil.

At events like the Cleveland Thyagaraja Aradhana, English is the default language that leads events. Yet, most performers and audience members speak in Tamil, even on stage. Telugu is audible but is often overpowered by Tamil at events. The history of Tamil is important to contextualizing the push for Tamil. Tamil has often been viewed as marginalized in greater India and also throughout its history (Selby, 2008). It is important 
to note that for centuries, Tamil Nadu was continuously occupied by outside groups that had profound influence on its customs and traditions (Kalidos 1976).

Tamil speakers account for $93 \%$ of the population in Tamil Nadu, making it the majority of the state and the fifth most spoken language in the country (COI, 2001). However, linguistically, Telugu-speaking authorities ruled Tamil Nadu for years (Selby 2008). This is in part why many of Thyagaraja's compositions, celebrated at the CTA, were written in Sanskrit and Telugu (Viswanathan and Allen, 2004). Yet, the connection to Tamil and the Tamil language was pushed as historically important. Tamil was not seen as the default, more sophisticated language, and for years, many felt that it was marginalized in the Karnatak arena (Viswanathan and Allen, 2004). Thus, after years of Karnatak concerts performed only in the languages of Telugu and Sanskrit, the Tamil Isai ${ }^{30}$ Movement in the 1940s, pushed for integrating Tamil into Karnatak culture (Viswanathan and Allen 2004). In today's performances, Tamil is now visible, but not a majority.

But, Tamil as a language was not just important to the classical music arena. Despite its status as the dominant language in the state, Tamil was a source of strife after independence. After India's independence, members of the Indian National Congress party governed the region of Tamil Nadu (then known as Madras State), which created a rise in regional parties that supported Tamil language against what was seen as Hindi-speaking domination in the nation (Chidambaram, 1987). The Tamil political party Dravida Munnetra Kazhagam (DMK) formed in 1949, promoted Tamil history and Sangam period literature, and fought for separatism (Chidambaram 1987). While Tamil speakers are the

${ }^{30}$ Isai is music in Tamil. 
dominant group in Tamil Nadu, they are not within the larger nation of India (Velayutham, 2008).

Stereotypes of Tamils as dirty, backward, or stereotypes of the language as rough or harsh persist in films and other mass media representations (Devadas and Velayutham, 2008; Jacob 2009). Devadas and Velayutham (2008) argue that cultural dominance as, for example, through the influence of Bollywood, creates a hegemonic nationalism and national identity of India, largely shaped around North Indian narratives, thus marginalizing counter-narratives and histories of the South. It is no surprise then that broader implications of regional difference are apparent in many efforts in Tamil communities to preserve Tamil culture, particularly aural aspects of spoken language and music. Sounds of language and music serve as markers of these identities and define them throughout events and performances. Though some events, like the 2016 CTA, display the importance of hybridity, other events still reinforce scale through language as seen in the 2016 Pongal festival discussed in Chapter 5. In NEOTS events, regional scale is used to distinguish clear boundaries of Tamil identity. Yet, in CTA events, hybrid identities like Indian-American, though deployed through a Tamil lens, are emphasized.

For many participants, speaking Tamil well is a source of pride for many and a sense of shame for others. Many described that they wished they spoke Tamil better, or that they felt that their level of Tamil affected the ability to live comfortably as a Tamil in the US. Others, fluent in Tamil, spoke the same of English, suggesting that they would be more comfortable if they could only speak English better. Participants often overlapped discussions of language and accent which I address further in Chapter 6 and 7. 
Accent

Accent is often discussed simultaneously with language. Accent, a part of language, involves both "tonal qualities" and "word choice" (Dave, 2013). For Dave (2013), however, accent is representative of a larger process of constructive norms and difference. Dave (2013) suggests that accents are generally compared to what is considered standard, normal speech and key to the process of "othering". In this section, I introduce accent's importance to interviews, building up to its significance in discrimination and "othering" much like Dave (2013) suggests, of Indian Tamil Americans. This end of this section bridges the discussion of sound and discrimination, further discussed in Chapters 6 and 7.

Accent was significant to both participant observation and interview data. Most participants described that accent was significant, though they thought of accent in at least four different ways. First, often those in the first generation or those who spoke Tamil more fluently, identified differences in Tamil accents. These Tamil accents were also indicative of more local scales. Participants described the Madurai, Coimbatore, Chennai, Tirunelveli and other accents as significant to more local identities within Tamil Nadu. They described these accents in two ways - how they are represented in films and movies, and how they identify local/scalar differences of Tamil Nadu. Many participants discussed how different accents were represented by Tamil cinema and how each film tries to replicate an accent based on the area in which the film is taking place. Some participants sent me clips of their favorite films where accents like Tirunelveli or Madurai were highlighted. Overall, participants acknowledged that accents were represented in films, but focused mainly on their personal experiences with accents and how they contributed to these more local identities. 
These accents seemed to indicate to some, certain cultures or characteristics of Tamil speakers. I've been using the term regional to refer to regions within India, so I will use the term local to refer to the regions within Tamil Nadu that participants described. Local scales within Tamil Nadu were highlighted through accent and quite significant. In fact, when discussing Tamil sounds, small-scale, more local identities were highlighted through language and accent. Many identified Tamil based on region and area in which someone was from, imparting them with not only with specific sounds, but also with characteristics. These identities had a more local focus than the broader, more regional Tamil identity that participants described. For example, Raman used accent to describe certain characteristics of Tamils:

The place where I am from is the Southernmost part, we have a special slang. In the North, we have a different slang. I would be able to identify 3 or 4 different people which area they are from their unique accent. This identifies which portion of Tamil Nadu like "yella" or "bee" kind of slang. In places like, Coimbatore, they give more respect... In Chennai, we can see lot of words from diff languages being used. Hindi, Telugu - some of those words get mixed.

Many described the Chennai accent and subsequently identity as more cosmopolitan, global, and hybrid - influenced by other parts of India and even the world. Because of this, it was also less respectful than other accents. Vijaya elaborates on this point. "Chennai is completely different than Madurai Tamil or Coimbatore..." She described that the Chennai 
accent has a lack of grammar and respect, while Madurai Tamil is very pure Tamil31. She said people joke about places like Coimbatore because they give so much respect to everyone, "even newborns." Participants, regardless of what part of Tamil Nadu they were from, generally suggested that people from Madurai speak the best and purest form of Tamil. Similarly, they also suggested that Chennai had the worst Tamil accent because it was mixed with so many other languages lie English and Telugu. Most people I interviewed from Chennai described this characteristic as funny and joked about it throughout the interview.

Yet, it was not always seen as a joke, but had real implications for some. Puran, for example, described how his Chennai accent, was associated with negative qualities, which he found frustrating. He described these experiences in relation to moving to another part of Tamil Nadu.

I am from Chennai. You are marked as a person from Madras. That also implies so many other things like as a person from Madras, you do not know respectful behavior. These are implied things. Associated things. So, the further associations are that you are impolite to others, and as a person from city, you are greedy. So, your sounds mark you as a greedy person, as an uncivil person... there are so many other associations. So, all these bundle up as the status that is you are a foreigner. We are different. You are different. You are othered. Without you being aware of it.

\footnotetext{
31 Vijaya is referring to the way that pronouns or verbs are constructed. In English, no differentiation is made, but in many other languages, distinctions are made between elders and those who are considered equals or younger.
} 
Many described the characteristics relating to the way that they spoke. Pandian also referenced characteristics when describing his origins in Madurai:

We all know that India is a very diverse nation so there are like 28 states which have 21-22 different languages. And they all have different kinds of characters which they cultivate in those regions. But when you zoom into that state, even in a single state, when you go to different districts you can see people exhibit different characteristics, speaking different dialects of the same language....I'm from Madurai, but by the way he speaks you can clearly identify if he or she is from Madurai or not.

He mentioned that there are both positive and negative characteristics associated with Madurai and people from Madurai. The language is strong, but people sometimes associate Madurai with violence.

Madurai has always been known to the other places in TN as a violent district or something like that, because I don't know, in the history we might have had some popular anti-social people. Also, people who would get into a fist fight more often than other people. Who could not control their anger or something like that. When you travel outside Madurai, it doesn't matter if you identify yourself as one of the people who cannot tolerate something, people will start to identify you as someone because you are from so and so place. That is one of the things that you get identified with. 
For Pandian, his accent reveals that he is from Madurai, yet he is also identified with characteristics and stereotypes. He says that not everyone makes these assumptions, but they are common associations. The local-scale identification of Madurai Tamil or Chennai Tamil brings with it, a plethora of associations that factor into the way one speaks. This is important to understanding levels and layers of discrimination and sound within the Indian diaspora which I will further discuss in Chapter 7.

Second, beyond the traditional scalar markers that created stereotypes or identified difference, I found that people identified particular accents with caste. In my previous research and current research, people often described these differences in detail. For example, people who were Brahmin would use the word "jalum" instead of "Thanni" for water. Those in lower castes would never use the word "Jalum". I will discuss this further in Chapter 7, but it is important to note that caste politics was seen as significant by many participants to both music, as discussed before, and accent.

Third, beyond differences between Tamil accents, people also discussed how Tamil changes in the US. This Tamil is no longer associated with a local spoken Tamil, but instead with a more global, hybrid and mixed Tamil. People who recently moved to the US describe that those who have been living here for a while or their children, don't speak Tamil in the same way that they might in Tamil Nadu. Much like the discussion on language that Pandian and Arvind commented on separately, morphing accents was significant to the shift in language. Pandian commented on this in more detail.

I mean if their Tamil got somehow mixed with English, and they start developing English accents of Tamil language because they have English accents. See the way 
construct a sentence in Tamil is different than the way you construct a sentence in English. When you start applying this rule to construct a sentence in Tamil you can easily differentiate that he or she has forgotten how to construct a sentence in Tamil. And is trying to make use of the same tone. You feel like he or she is speaking English while they are actually speaking Tamil.

This observation was not confined to first generation participants but was noticed by second generation participants as well. Kumaran, for example, said that he definitely noticed a difference in the way Tamil was spoken growing up versus when he visited India. “Even for my parents, their Tamil has devolved into liberal English. They morph into a Tamil English thing...That is the Tamil that I'm used to - my parents watered it down. I can understand my family for the most part. I can't understand formal Tamil." Participants like Kumaran, Durga, and many other second-generation participants explained that the Tamil that they heard growing up, was very much filtered by English. They mentioned that when visiting Tamil Nadu, it was obvious that they had American accents, but many family members and strangers accused their parents of developing American accents as well. Their accents became a marker that, though they might look Tamil, they were no longer purely Tamil, but instead were American and easily identifiable as NRIs (non-resident Indians). Accent was a marker that their identities were blurred and hybrid, not fitting fully into any categories. They were no longer "purely" Tamil but had been influenced by their time and life in the US, even despite any efforts to maintain connections to "pure" Tamil. Accent was both a marker of otherness and hybridity. 
The fourth conceptualization of accent referred to how people described the internal complexities of living with changed accents or being aware of their accents when in new locations. Thus far, I have discussed Tamil accents in the context of Tamil communities. However, accent was significant to experiences with being Indian and being American living in the United States. For example, both Matthew and Nazeem described complexities of living with a Southern accent in the United States and then relocating. Matthew, when he first came the US as a 9-year-old, was made fun of for his "Indian" accent. Later in his life, after developing a Southern, Alabama accent, was then made fun of for his Southern accent when he moved to the Northern parts of the US. I elaborate on this story a bit more in Chapters 6 and 7, but it is important to note, that changing accents was significant to many participants.

\section{Summary}

While sound overall was significant, the ways in which it was significant varied based on sound type. Environmental sounds often linked with memories and food, while accent was often discussed in relation to discrimination. Music was also associated strongly with memories and emotions as was language. Language was also described in relation to discrimination, but also in a more visceral, abstract way. Environmental sounds, music, and language were all described in a very visceral, emotional way at some point in most interviews. Yet, accent, was more often than not, associated with more tangible, lived experiences. Most importantly, as I have noted, it was significant to the ways in which participants perceived and/or experienced discrimination. This is a significant discussion that I will delve into in the next chapter. In Chapter 6, I will elaborate on how music, 
language, and accent influenced or shaped participants' experiences with discrimination. In Chapter 6 and 7, I will demonstrate that discrimination is not just experienced through visuals, but also through sound. 


\section{Chapter 6: Discrimination and "Othering" in the US for Indian Tamils}

In Chapters 4 and 5, I outlined how scale - hierarchical and sometimes multiple hybridity, and sound informed participant identities. I first focused on the nuances of scale and hybridity in Chapter 4 and then on the links between identity and sound in Chapter 5. I showed how environmental sounds, music, language, and accent added to the experience and representation of identity, both through participant observation and interviews. In the last section of Chapter 5 on language and accent, I briefly discussed sound's importance for participants discussing discrimination, which I develop further in this chapter along with the intersection of scale, hybridity, and discrimination.

First, to revisit scale and hybridity, participants often theorized scale as hierarchical and rigid - global, like world-citizen, national, like Indian or American, regional, like South Indian or Tamil, local, like Madurai or Chennai, and or even more local with a specific town or village. At the same time, in other moments, these scales were blurred and hybrid. These scalar identities were hybrid at it was not only because they blurred or were in-between at times. In fact, many times, they were hybrid because they were products of a postcolonial legacy of diaspora. In other words, the binaries established by colonialism made some identities seem binary or opposing identities, when they were in fact, hybrid, fluid, hyphenated, and blurred. As Antonsich (2018) describes, local and global scales (and those in-between) of identity, are intertwined and blurred, yet also at times hierarchical. They are hybrid, in-between in moments, and hierarchical in others, yet all dependent on moment. 
These scales also link identity and discrimination as I will demonstrate in this chapter. Participants who initially scaled up to identify as "global" or "world-citizen", scaled down to identify as Indian, Tamil, American, South Asian, etc. when referring to instances of discrimination in the US. Or, participants who identified with local identities, scaled up to identify as Indian or brown when describing discrimination in the US. Sometimes, they identified with large scales and other times with small scales, depending on the context. Even those who said that they did not really identify with national or regional identities like Indian, Tamil, or American, but instead with more large scale identities like global, firmly identified with smaller scales when describing instances of discrimination. They suggested that these identities were the cause of why they faced discrimination. Some participants who did not see themselves as Indian or Tamil in the first part of the interview, for instance, described that they were at times marginalized because they were Indian or Tamil. Even if others discriminated against them because they misrecognized them as 'Middle Eastern,' many participants suggested that they faced discrimination precisely because they were Indian or Tamil. They linked these identities often to brownness, but which included multiple markers of difference.

Brownness, as many scholars suggest, is not just based on visuals, but on a set of characteristics that differentiate from being the standard, white norm (Bhatia, 2007; Dave, 2013). Brownness can be multi-sensory (Simonsen, 2010; Dave, 2013). For example, accent, language, and in some cases even taste and smell were a part of these discussions. Brownness also became scalar in that it referenced a supranational group of non-white individuals living in societies where whiteness is viewed as standard or normative. Brown could include those who were South Asian, but some participants also described 
brownness as connected to those from Central or South America and even the "Middle East." In other words, brownness surpassed South Asian in reference to scale in a larger, somewhat global scale of brownness.

Sound was also especially noteworthy. For example, I would ask if participants had ever experienced discrimination. Those who often responded with no, changed their answer, or described an experience after I brought up language or accent. Most participants who initially denied experiencing discrimination had at least one example of discrimination or othering regarding accent or language. They did not always associate these with discrimination, however, and often couched these examples under the idea that they needed to improve their accents or language and it was their fault. Yet, they did describe these incidents as making them uncomfortable.

It is important to note that discrimination had different meanings for each participant. Discrimination for some, was physical - a hate crime or an act of physical violence. For others, discrimination was active - they were denied opportunities because of their identities. For some, they described it as a form of "othering" - they were looked at, treated differently, spoken to in a derogatory way, or scrutinized because of specific identities. In many ways, othering was linked to microaggressions. From this point forward, when referring to othering, I also include microaggressions. As Joshi, McCutcheon, and Sweet (2015, p. 300) describe, "Racial microaggressions are not overt racist acts and sometimes occur without the perpetrator or even the victim being aware of them." Microaggressions were directly identified as a form of discrimination by some participants. Joshi, McCutcheon, and Sweet (2015, p. 305) are clear that microaggressions, ranging from "overt" to "subtle" create emotional turmoil for many people of color. As the authors 
suggest, microaggressions can be so subtle at times, that perpetrators can deny them, causing victims to "second-guess" themselves while constantly questioning the validity of their experiences.

While "othering" for was banal compared to physical acts of violence for some, it was not for others. "Othering" associated with discrimination created a sense of fear, emotional turmoil, and sometimes a manifestation of physical ailments from psychological trauma. Many researchers have documented the psychological effects of discrimination and othering on bodies (Carter, 2007; Carter, Sant-barket, Carter, \& Sant-barket, 2014; Franklin, Boyd-Franklin, \& Kelly, 2006; Joshi et al., 2015; Lee \& Ahn, 2011). Several studies in psychology have examined how discrimination has emotional, psychological, and physical effects on Asian Americans and the ways in which they identify (Tummala-Narra, Alegria, \& Chen, 2012; Yoo \& Lee, 2005). In fact, many of these studies show that discrimination significantly affects identity and how Asian Americans experience daily life based on these identities (Yoo and Lee, 2005).

In this chapter, I build on and analyze discrimination in its many definitions outlined by participants - physical, active, and even as a form of "othering". I weave discussions of scale, hybridity, and sound into a larger discussion on discrimination and identity. In general, most participants viewed discrimination and othering in the US through largescales like South Asian, Indian, or desi ${ }^{32}$ rather than regional scales like Tamil, local like with a specific village, or even broadly as global or world citizen. In fact, participants who did describe themselves as global or world-citizen, did not reference these identities when

32 "Desi" refers to a person of South Asian descent and originated from the Sanskrit term deśa meaning land or country (Oxford Dictionary, 2018). 
describing discrimination. Sometimes, participants would interchange "brown" with Indian or desi. Only when describing discrimination within the Indian community did most participants shift to a smaller, regional scale like Tamil. Even more so, when describing discrimination within Tamil communities, participants then identified with with more local scales like Madurai Tamil, or from their village (see Chapter 4). Smaller scales were more important for participants to identify discrimination within Indian communities and larger scales were important to identifying discrimination within the US.

I found that discrimination and othering was connected to scale and hybridity, but also to whiteness. For example, in the context of discrimination and othering in the US, participants viewed themselves more broadly as being brown or from India as opposed to American whiteness, even though in other circumstances, they may have viewed themselves as more closely linked to whiteness in opposition to other minority communities. Whiteness is significant to discussions of discrimination in the Indian diaspora as well. In Chapter 7, I connect whiteness to the Indian diaspora, but for this chapter, I discuss whiteness in relation to the United States.

Hybridity was important because, although participants identified identities in a very hierarchical scalar way at times, in other moments, they described very blurred or even in-between identities. For example, many participants described that they experienced discrimination because they did not fit fully into any category, i.e. what popular culture, Sangams, Indian communities, family, peers, governments, and even academics represented as Indian, American, or Tamil. They felt in-between these definitions. These were also connected with sound and more specifically, music, accent, and language, as shown in Table 1 in Chapter 4. 
Throughout interviews, participants discussed discrimination in two ways - first through experiences within the US and US politics, and second, through internal complexities of broader Indian communities. This chapter focuses on the relationship to the US, while Chapter 7 focuses on discrimination within Indian communities. I have provided a figure to demonstrate these complex relationships and provide an overview of the discrimination discussions ahead.

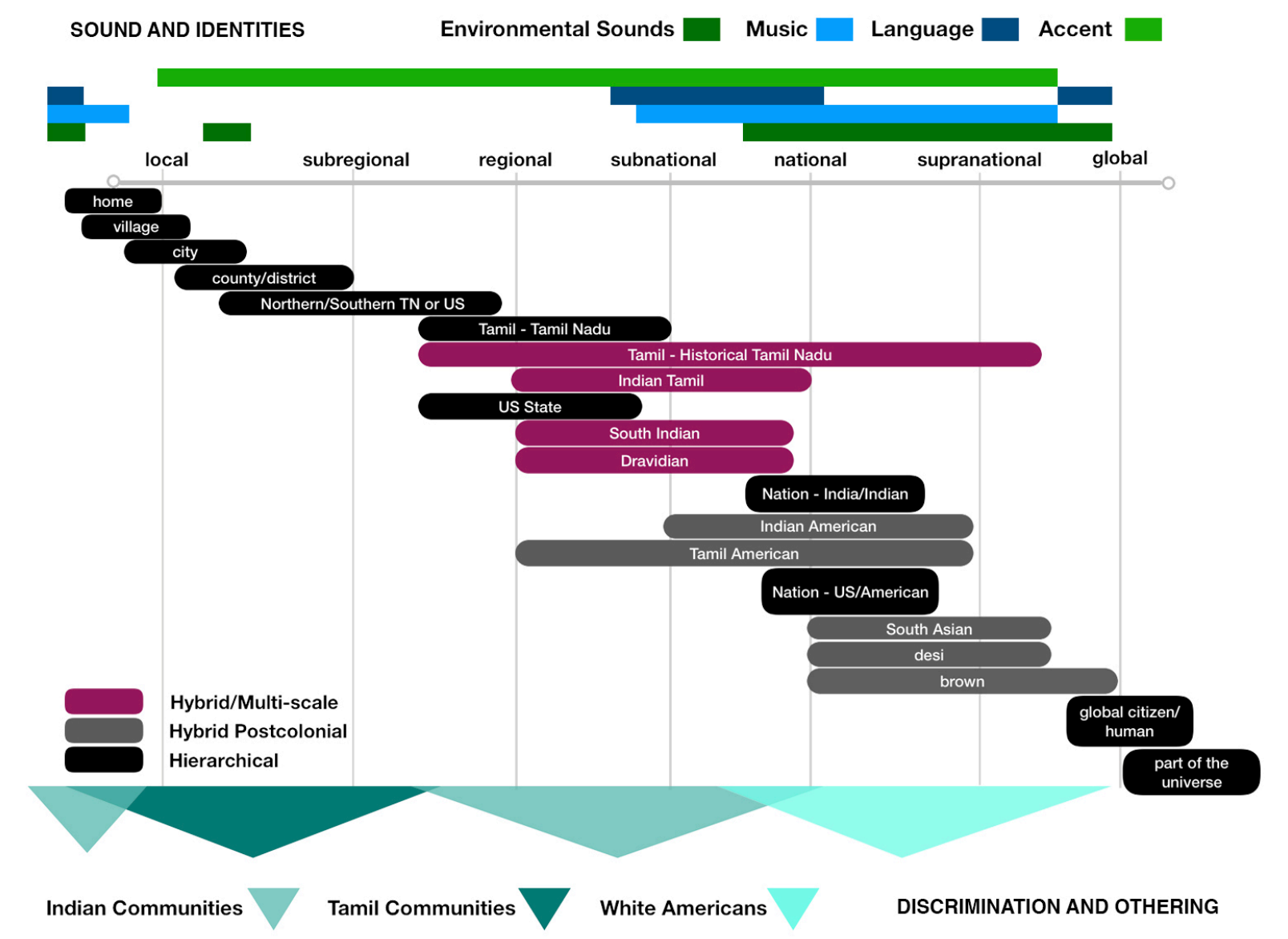

Figure 7: Ways that discrimination and othering intersect with spatial/hybrid identities and sound*

*Note: Though shown on this chart, environmental sounds rarely intersected with discrimination and othering. Music, accent, and language did, but environmental sounds did not. Areas of discrimination are shown below. This figure demonstrates the various identities that participants 
described discrimination and othering with respect to Indian, Tamil, and American communities and the types of sounds associated with these instances of discrimination or othering.

\section{South Asians and stereotypes in the US}

How identities are represented can shape stereotypes and how people experience these identities within specific contexts (Dave, 2013; Hopkins et. al, 2017). For example, South Asians post-9/11 are more likely to experience discrimination than before 9/11 according SAALT (South Asian Americans Leading Together), an organization that focuses on policy and action on South Asian discrimination. Safran and Sahoo (2008), Hopkins et. al (2017) and other scholars have also noted the rise in hate crimes against South Asian communities that correlate with the rise in Islamophobia post 9/11. But as SAALT and many others acknowledge, violence against Asians is not new and occurred pre-9/11. It is more pronounced with increased Islamophobia and anti-immigrant sentiments following post-9/11 shifts in immigration and security rhetoric (Safran and Sahoo, year; Bhatia, 2007; Gökarıksel and Smith, 2016; Hopkins et. al, 2017)..

SAALT (Sridaran et al., 2017) indicates that while Hindus, Christians, and other South Asians do experience hate violence, usually from misrecognition, those most likely to experience hate crimes are middle-class Muslims or Sikhs in the South Asian community. Hate crimes against South Asians, particularly Indian Americans in the United States, have increased post-2015 (Sridaran et al., 2017). Ninety-four percent of hate crimes since 2014 were based on anti-Muslim discrimination or misrecognition (Sridaran et al., 2017). SAALT (Sridaran et al., 2017) found that in the US in 2015, there was a 67 percent increase in hate crimes since 2014, reaching some of the highest levels since 2001, post-9/11. I interviewed 
Lakshmi Sridaran, SAALT's director of national policy and advocacy in October of 2017. She explained that the number of hate crimes has spiked since the election of Trump, surpassing the numbers of 2016 as of October 2017. In other words, as Sridaran indicated, they have entered a period in which hate crimes against South Asians may reach unprecedented levels. SAALT more recently reported that in 2018 (Modi, 2018) hate crimes against South Asians have likely surpassed 9/11 levels, with an additional 64 percent increase following the election of Donald Trump. One of out every five hate crime incidents made direct reference to Trump, his campaigns, or policies, while others were connected to a rise in white supremacy groups or active references to "brownness", immigration, Islamophobia (Modi, 2018).

SAALT (Sridaran et al., 2017) also expresses concerns that many hate crimes go underreported and that these are about 20-30 times higher than reported numbers. SAALT (Sridaran et al., 2017) attributes this to multiple factors including distrust of law enforcement. As I observed in my interviewees, underreporting may also be influenced by the fact that many people do not think they experience hate crimes, or they explain that things can be a lot worse, so there is no reason to worry about these incidents. Many participants described that their experiences were not that bad, or with first generation participants, described that they deserved some scrutiny precisely because they were immigrants.

South Asians have historically experienced discrimination in the US even pre-9/11 (See Chapter 3). Previously, Indians experienced stereotypes such as the 'model minority', which silenced underlying discrimination that many Indians faced. Simply put, governments, media, and other dominant narratives initially represented Indians 
convenient store or hotel owners, but began to discuss them as doctors, lawyers, or highlyeducated people with immigrant success stories (Bhatia, 2007). But, in 2014, India had the fourth largest source of unauthorized migrants to the US who do not occupy upper-class roles in US society (Passel \& Cohn, 2016). Conversations on Consideration of Deferred Action for Childhood Arrivals (DACA) and Deferred Action for Parents of Americans and Lawful Permanent Residents (DAPA) are not usually associated with Indian Americans, but SAALT (2015) estimates that 41 percent of undocumented Indian immigrants would be eligible for these programs that grant deferred legal action renewable every two years and authorization for work permits. SAALT (Sridaran et al., 2017) also estimates that this will significantly affect many Indian Americans in the United States, but because the model minority stereotype often masks Indians, issues associated with other minority communities, go unnoticed in relation to South Asians.

Both undocumented and documented Indian immigrants face challenges with recent political rhetoric. In 2017, US Citizenship and Immigration emphasized "targeted site visits" to detect "fraud" - suggesting that H1-B visa fraud is rampant across the US (USCIS, 2017b, 2017a). This is not just a product of misrecognition, but also one of blatant targeting as well. People have created websites like "Save IT Jobs" (see Introduction) which has since been taken down, to target Indian Americans, or any minority that they fear might take away "American" jobs from white Americans. New policy initiatives and popular culture references have emphasized messages of Indians stealing jobs, fraud, and disrupting American culture.

American popular culture has stereotyped Indians as threatening for stealing IT jobs, running convenient stores or hotels, stealing jobs as doctors, as outsiders trying to 
take over America, or alternatively, being funny sidekicks who supplement or serve to boost the narrative of white characters (Dave, 2013). Dave (2013) examines how the "Indian" accent as an aural marker shows non-white "otherness". Dave (2013) points to characters like Raj in The Big Bang Theory, who serve as the stereotype for the H1-B visa Indian coming to the US. His contribution to the comedy is his "Indian" accent, reinforcing the stereotype of being nerdy, techy, and a sexually undesirable "other." She describes that the Indian accent is associated with these undesirable characteristics, which media representations in the US continually reinforce. Even in the show, Raj's character never changes or develops, but continues to reproduce and reinforce a typical punch line for American audiences of stereotypical "Indians."

Dave (2013) draws parallels to the representation of Indian Americans through the character of Apu on the Simpsons. She describes that this is typical of many Indians, represented in Hollywood. What makes Indians funny, tolerable, or non-threatening is the accent that signifies the undesirable or "other" characteristics that do not conform to white standards or norms. After all, as comedians like Aziz Ansari and Hari Kondabolu have discussed in various comedy sketches, Indians have to be marked as Indian, they can't play Americans because they aren't white enough. Hari Kondabolu, recently released a film (Melamedoff, 2017), "The Problem with Apu" commenting on the "Indian" accent and stereotypes that he argues affected many Indian Americans living in the US in the last 30 years. He suggests that Indian representation became funny, a punch-line, and after 9/11 even became represented or conflated with terrorism. He suggests that Indians in media could not take on roles as Americans, because they were continuously deployed in media through these incessant "Indian" stereotypes. Tying this to hybridity, these reinforced 
stereotypes suggest that binaries between the colonizer, in this case the whiteness in the US, and the colonized, Indians, persist in many media outlets in the US.

In my own research, many participants reflected these sentiments and even commented on how they did not like the representation of the "Indian" accent. Some said they did not know how they felt about it. Durga, for example, described that it depended on who she was with at the time. "If we are watching TV, if there is an Indian accent there for comedic effects, I don't know how to feel. Depending on who I'm watching it with. If that person is Indian I can laugh. If person is not, I feel like I need be an ambassador for Indians." Accent was not just something that my participants saw represented on TV. It was and is significant to how many of them experience discrimination and stereotyping.

\section{Constructions of "other"}

As mentioned in the beginning of the chapter, discrimination had multiple meanings for participants, from being "othered", including microaggressions, to active hate crimes. In all such definitions, participants were at least somewhat concerned with discrimination. I broke discrimination into five sections. First, for readability and second, because participants seemed to think about discrimination in five ways. These were often discussed as related to significant events like the election of Donald Trump or 9/11. Yet, they were not always connected to a specific time period, but rather themes like Islamophobia that emerged after significant events.

First, I situate discrimination with an important factor of geographical location often referenced by participants. This was important to how participants discussed othering and discrimination in the US. The second section discusses the implications of 
Islamophobia, anti-immigrant sentiments , non-whiteness, the rise of white supremacy, and/or stereotyping that culminated with the election of Donald Trump - something that nearly all participants referenced. Responses ranged from slight concern to extreme concern. Some actively experienced discrimination like having someone threaten them while others worried about it, describing more of a psychological trauma. Many participants, though, described in detail, instances where non-Asian, often white Americans caused them psychological or emotional fear after or during the election.

The third section is based on themes that participants referenced in relation to discrimination post-9/11 and implications of brownness. Those who lived in the US before and after 9/11 referenced a change in rhetoric and increased fear of discrimination. Participant interpretations of post-9/11 effects varied. Most who had lived in the US preand post-9/11 said that they noticed a difference in how they were treated. Those who had not lived in the US prior to $9 / 11$ did not have such reference points. Those who lived in the US longer generally shared more stories than those who had lived in the US for a shorter amount of time. This does not mean that those who lived in the US for a shorter time experienced fewer instances of discrimination, but perhaps felt less comfortable sharing their experiences. Another key factor was if a participant lived in or grown up in the US before and after 9/11. Those who experienced living, and often growing up, in a post-9/11 US were more likely to share instances of discrimination or othering with me. The types of discrimination and what counted as discrimination varied between participants. Second generation participants framed discrimination more around the concept of othering, while first generation participants more often framed discrimination as an active hate crime. 
Fourth was pre-9/11 discrimination, revolving around Orientalism and "thirdworld" stereotypes, which participants referenced the least in my interviews, but was still overall significant to conversations on discrimination. These stereotypes were like those that Jazeel (2006) describes of the Sri Lankan Women's Association in the UK, described in Chapter 2. Though focus changed post-9/11, these stereotypes nevertheless persisted post-9/11 and post-Trump election. Again, these categories serve as reference point to specific types of discrimination, not necessarily the events themselves. This language was used by participants and thus, I have used it for the analysis as well.

I am not providing an extensive overview on these points of discrimination in terms of addressing temporal discrimination. My purpose in outlining these is merely reflecting the ways in which participants divided the types of discrimination they felt. In other words, the discrimination they felt was loosely connected to events such as $9 / 11$ or post-Trump election, but more often was described as a type of discrimination or othering such as Islamophobia, orientalism, and/or anti-immigration and white supremacy. Many of these categories blended and blurred, but for the purpose of readability, I have separated them.

Finally, I discuss the web of discrimination, which shows how discrimination is fluid and situated through multiple life experiences. In many ways, these five sections overlap, but for clarity, I have separated them. I organized the analysis into these sections because participants recognized these as significant events and framed their discussion of themes like Islamophobia, xenophobia, and "third-world" stereotypes around the events of 9/11 and the Trump election. However, these sections serve more as ways to categorize these different types of discrimination. Much of this overlapped and discrimination was not discretely confined to specific time periods. In fact, the discussion of discrimination was 
much more fluid and overlapped. Discussions of scale, hybridity, and sound are weaved throughout.

\section{Importance of geographical location}

I found that most participants experienced more othering or discrimination in rural areas rather than urban areas. This was also related to diversity. Participants described that they experienced discrimination, othering, or stereotyping in less-diverse places, which were often more rural. This is not new. Many scholars, even outside of geography, have found that geographical location has been significant to experiences of discrimination in many “Western” countries (Chacón \& Davis, 2018; Fennelly \& Federico, 2008; Neal, 2002). Neal (2002) argues that rural areas have been represented as white through literature, film, and other forms of discourse, which she then argues, are reified in daily practices and experiences. Fennelly and Frederico (2008) argues that in the United States, nativism expressed as whiteness, is heightened in rural areas, especially in recent decades when immigration and anti-blackness has become central to political debates. Much research surrounding rural areas and racism has focused on the effects of immigrant populations moving to rural areas for agricultural or plant-processing work (Chacón \& Davis, 2018; Fennelly \& Federico, 2008; Winders, 2005). Rural areas have also made headlines most recently with Attorney General Jeff Sessions, who is also known for his controversial rulings in Alabama that reified anti-black and racist policies as well as his anti-black comments and jokes such as justifying the existence of the Ku Klux Klan (Phillips, 2017). Sessions brought rural towns like Albertville, Alabama in the political spotlight as 
examples of how immigrants ruined wages and quality of life for white "Americans" (Glass \& Meek, 2017).

So, it was not surprising that most participants described incidents of discrimination happening in rural areas or in areas where they were singled out as a visible or audible minority. In other words, if they were the only brown person visually, aurally, and through other senses, other Americans, often white, pointed this out. These incidents of discrimination varied from being pointed out as an "other" to verbal assaults or threats. When looking to organizations like SAALT though, Sridaran (2017) suggested that their reported hate crimes came from urban areas. But, this does not necessarily reflect the frequency of hate crimes, but the number of people reporting hate crimes. As Sridaran (2017) indicated, knowledge of organizations like SAALT are much less likely in rural areas.

Those growing up in more rural areas, what some described as "non-diverse" areas, or Southern states, were also more likely to experience instances of othering or discrimination than those who lived in more diverse environments like college towns or large urban centers. I interviewed participants from a variety of areas - urban, rural, university, small town, large city, mostly connected to the specific sites of Morgantown, WV, Northeast Ohio and Northern New Jersey. But, these participants had also lived in multiple areas of the United States - from the West Coast, South, to the North and East coast.

For many participants, even if they had never lived in rural areas, rural areas were associated with being white and white culture and they became the visible and audible minority. Anusha describes her experiences growing up in a rural area. She said she could 
not exactly describe why it was significant, only that it made her very aware that she was not white or that she was different from the norm. It affected her identity and her life as she describes:

So I grew up outside of Washington D.C. So like from four to the first year of high school we lived in a town called Germantown, so when I was growing up it was for young families, and was just getting developed so it was still pretty rural compared to the suburbs of D.C. It was pretty white. Realistically speaking, it was kind of diverse... but I felt that the culture was still very white. And strongly so and there was an urge to preserve that. In some ways that I can't necessarily put my finger on, that was very present for me, like very pivotal parts of my life...Like I remember walking down the street with my brother, at the time, I thought they were adults, they (referring to some people in the neighborhood) yelled out the window and called us the $\mathrm{N}$ word.

Although many participants described othering and discrimination in rural areas, it was not limited to these areas. Even in college towns and large urban centers, participants, though fewer described incidents, still described concern with discrimination. They worried that eventually, hate crimes and racism would become open and blatant, especially with the rise of the Trump administration. For example, Preeti, who grew up in New Jersey, describes how her experiences have shifted depending upon where she was in the US. 
I grew up in New Jersey which is a pretty diverse state. I've had some microaggressions or whatever you want to call it. It is just kids being assholes. I did get some of that teasing - like Indians smell bad or comments about my food. That kind of stuff, growing up I got some of that. Generally, it wasn't that terrible. My experience as an adult traveling to other parts of the country, is somewhat lessfriendly. It is an uncomfortable staring like they never seen you before. One time, my friend and I were in rural Pennsylvania, not like State College, like the confederate flag-waving areas. We wanted to know where to get flowers for our friend and no one would talk to us. She was black and I'm brown so two people of color, we were just completely ignored. One time we were eating, they looked visibly scared like they like moved away from us. They were visibly afraid. It was kinda funny to me but also like, is this real? I've had isolated experiences like that usually in the south or in a really rural area but for the most part, I've been in pretty diverse areas I've generally been in more accepting communities, but definitely not as bad as others probably had.

Many participants beyond Preeti described the importance of location in relation to discrimination and othering. I discuss this further in the next few sections.

\section{Recent Islamophobia, xenophobia, and whiteness}

When I asked about discrimination, almost all participants mentioned the Trump election at least once. Some discussed the implications for 1-2 hours and other, 1-2 minutes. Regardless of the discussion length, the Trump election was significant to many 
participants in some way. From my interviews, most discussed Trump and the new administration very negatively. Many participants described disdain and frustration with the events and racial tensions that they had experienced post-Trump election. But, even if a participant disliked the current administration, their day to day experience was quite complex and often not clear-cut. During informal conversations, some participants discussed political leanings of other participants that I had interviewed, pointing out that so and so voted for Trump, supported Trump's policies, etc., or so and so "voted for Trump and now regrets it." Even with interview data, the discussion on this subject was complex and emotionally-loaded for many participants.

Not all participants directly encountered discrimination or racism post-Trump election but knew others that had. Maari describes what happened to his friend after the election" My friend had the worst experience after Trump got elected. He was filling tanks and the guys shouted at him go back to his country or else he'll make him." This concerned Maari and he said that he hoped that he would not run into anyone racist like his friend did because he is Indian. Arvind also described that he was worried about discrimination as he had heard that it had happened to others. He said that especially after the shootings of Indian Americans in the US, he became concerned.

I was so worried about my son and grandson. They go to Tamil school. Now in front of the car we have unmarked police protection. It would be a good target for people because there are a lot of Indians gathering. 
Both participants described fear of discrimination and active violence. It is also worth noting that the way in which they described themselves in relation to the regional scale of Tamil changed. Earlier in conversation, Arvind referred to himself as Tamil, but when discussing discrimination in the US, he began to use Indian and Tamil interchangeably. The same was true for participants like Maari. Maari initially identified locally from Trichy but began to refer to himself more broadly as Indian when discussing discrimination. The use of these terms often surrounded the fear that participants had of discrimination. Both Arvind and Maari said they worry that something will happen. Maari, though, said that he has experienced discrimination before and this is because he is from India, not necessarily because he is from Trichy.

He began to identify on the larger scale of Indian in relation to discrimination in the US. This could be for a variety of reasons. As one participant described, identifying as Indian, South Asian, or even brown instead of Tamil when threatened in an unwelcoming country is a way to establish solidarity within a larger group. In other words, Indianness or brownness creates a safe space with others who experience or understand the same issues. For some though, it was simply an acknowledgment of their experiences living in a very white society and identifying as these things was necessary because "white people would look at you that way". This was an acknowledgement that Americans scale up and cannot distinguish nuance or diversity within brown communities. So, in these instances, because other Americans were homogenizing these identities, participants also homogenized identities. When discrimination was projected upon them, it was at the scale of the nation, or supra-national like desi or South Asian. As Raj described in Chapter 5 of Americans, "they know little geography of India." 
Maari says that he has not experienced anything in a directly violent way. He describes it as connected to both the way he looks and the way he speaks. "That happens to me a lot of time, why is your English good? I studied English as a first language in India too. These questions are generally asked by people who are above 50 because of the stereotypes." Aural markers were significant to how people interacted with Maari and the ways in which he felt marked as "other." Maari says that these stereotypes persist throughout the US, especially with "older" Americans. He says he is somewhat apprehensive about the Trump era because of these stereotypes in regard to language, accent, and other characteristics. For many like Maari, language, accent, and visuals signify certain characteristics, mostly negative, that others project onto him through stereotypes. As Kanngiser (2012) notes, language and accent inform the way that people hear and as Haldrup et. al (2006) suggest, these can influence and shape experiences of discrimination.

While Maari describes accent and language as significant, he also describes incidents of how he was misrecognized. He points out that what happened to him was not as significant as what happened to his friend after Trump's election. He does not always make a distinction between post-9/11, pre-9/11, or post-Trump election and says that stereotypes or discrimination resurface, depending on the political climate. But, he still says that these are significant points to how the treatment of Indians in the US has changed over time. He ties his experiences to the post-Trump election era, but says that he has experienced it before the election, during post-9/11 rhetoric:

I've had people talk to me as if I'm an Arab or from the Middle East. That always happens. One thing I believe in is that we cannot change people or the way people 
look at us. Not just in America. There are racist people everywhere. I'm not going to change them they are not going to affect me. The media portrays is worse than it is. I expected it worse when I first came. When I travel to California or South to Florida or Texas that is when it is a little worse. Not in Akron. There are a lot of issues in Texas.....

Like Preeti and Anusha, he also connects these to geographical location. I ask him what he means by issues in Texas. He elaborates:

Well, there is people who always, let's say you are travelling in a bus, there is always somebody who will get up when you sit next to them. I have also seen ones in flight when I was coming back from Dallas. A person requested another seat when I sat next to them. I'm a big dude, so maybe they wanted a different seat with more space. I don't know. So, it does happen a lot. Same thing happens in India but in a different way. Only in India, they won't shoot you ${ }^{33}$. Like they would here. It is always like when there is an industry or University center there is not much racism because they know there will be a lot of different people. So those small counties, there are always issues. Though Dallas is a big fricking city and there are still issues.

For Maari, geographic location is also important to when and how he experiences discrimination. For Maari, while discrimination is an issue, he was not as directly

33 Maari was referring to the 2017 shooting in a Kansas bar of two Indian Americans, described in the introduction. 
concerned about it. Maari's view of discrimination was that it happens and sometimes a lot, but nothing can be done about it. He indicated that his experiences with discrimination varied, but geographic location matters. California was on his list of places he experienced discrimination, but other participants had described California as a place where they experienced fewer instances of discrimination. But, each participant's life experiences contextualized their views on discrimination and the part of the state that they visited. Some had visited very urban areas, while others had been to more rural areas. Texas, overall, was not a surprising answer to my question regarding discrimination. Many participants mentioned discriminatory incidents in Texas or places in the South. Often, these related to accent, looks, or markers that made them different.

Matthew, raised in Alabama, describes that he had heightened concerns after the 2016 election. He no longer lives in Alabama but considers it a significant part of his experiences with identity. He equates "the South" with more inwardly racist behavior.

I have not experienced anything personally...I'm sort of sheltered from that. Although, certainly I get into political arguments at work. It isn't anything racerelated... but I certainly worry about it. I worry about the trending of more outward racism. I remember watching a documentary about how there is this underlying racist behavior... Southerners are great at that. Outwardly they are nice to your face, but inward they are pretty racist. I am making a general statement. Not saying everyone, but I worry about more inward racism becoming more outward. There are really smart people and they voted for Trump and it blew my mind. 
Matthew's experiences growing up in Alabama, described later in this chapter, informed his views with the 2016 election. So, although he did not experience any direct violence, like many participants, he worried about it and about the implications of justifying and normalizing racism, which he tied into his prior experiences. His experience with discrimination post-Trump election was more psychological. SAALT and other institutes often focus on direct violence and reported hate crimes. Yet, for many participants who had not experienced direct violence following the Trump election, psychological violence was much more significant.

Many participants, while they did not personally experience hate crimes postTrump election, describe the psychological issues associated with Trump's election. In other words, their discussion of discrimination focused on emotional impacts and the fear regarding discrimination. Bharathi, who came to the US in 1971, identified with a regional identity of Tamil. But, when discussing discrimination, again referred to herself with as national identity of Indian within the context of the current political climate. She said that the current political climate has affected her emotionally and she feels the effects daily. It is not something that she can just put out of her mind but is always with her.

The current climate... that, I don't even want to listen to anything. The more I listen the more I aggravate myself so I don't want to listen. Every day, I hear very hurtful things and sayings from them (Trump administration), so it is better not to listen. The previous presidents I never had this kind of emotional disturbance. He is really disturbing me emotionally. I want him to resign, but I know it isn't going to happen. 
This has caused her a great deal of psychological stress which she links to discrimination. She said since the election, she has a lot of anxiety and sometimes cannot sleep at night. I interviewed her in October of 2017, almost a year after the election. She says that although she is not as upset as she was initially, the feelings have not subsided. She worries often about the future and state of the country. Because she is Indian, she worries about her family, her friends, and other immigrants in general. She describes that current administration is attacking her and her identity as an Indian Tamil, especially the anti-immigration rhetoric. The Trump administration's immigration ban in February of 2017 especially caused her a significant amount of stress and worry. She says that when her husband turns on the TV to watch the news, she has to walk out of the room. She says, "I can't listen. It is too much." The country that she came to so many years ago is now turning on her and her family because of this administration. She said that she and her husband once felt welcome as Indians in the US, but do not feel this way currently. She initially described them as Tamils, but when discussing current issues with discrimination, shifted to a national lens of Indian.

Many participants brought up the emotional and psychological impacts of the Trump election. Earlier experiences that participants had or representations of their identities that they saw in popular media often influenced their current views. The Trump election affected some participants slightly, and others much more. Some, in fact, did not even want to talk about the election because they said that they still could not face it a year later. Much like what SAALT suggested, religion was a significant concern for some participants, especially those who identified as Indian Tamil and Muslim. Being Indian Tamil was significant, because it made them "brown," but being Muslim added another 
layer to the way that they experienced identity, especially during periods of heightened Islamophobia. Brown became a scale used interchangeably with Indian, South Asian, desi, Tamil, and others. Brown encompassed all these scales and was used interchangeably with large-scale identification. Simply put, brownness became synonymous with South Asian, desi, Tamil, Indian - almost exhibiting a hybrid, multi-scale discussion of identity. Brownness could be hybrid at times, blurring all of these identities, but also remaining inbetween them. Brownness could also be multiple, encompassing all of these identities simultaneously. The distinction between Indian and Tamil, which was important to lives and experiences, became less important during instances of othering or discrimination. Othering and discrimination cultivated a more hybrid understanding of identity that involved a blurring of identities - such as religion and brownness.

For example, Fathima and Suhail are siblings. I interviewed them separately, but they both expressed their concerns about being brown and Muslim in the current political climate. Both Suhail and Fathima identified as Muslim, American, and Tamil, but were especially concerned about being Muslim and brown after 2016 election. Fathima came to the US when she was 6 months old, while Suhail was born in the US. They grew up in Northeast Ohio. Suhail says that as a Muslim American he worries when he watches the news and sees a terrorist attack. He says he thinks, "Please God, don't let it be a Muslim." Though, he says that compared to Europe, the US is doing much better in terms of handling Islamophobia. Many participants often compared themselves or their situations to others who were not as fortunate or who experienced racism more than they did. Often, I found that these participants would make their experiences seem less important when compared 
to others. Maari, for example, continued to compare his experiences to his friend's experience with racism, suggesting that his friend had it worse.

Fathima, did not compare her experience to anyone else. She says that being Indian growing up in Ohio was hard, but being Muslim and brown is even harder, especially now. She says, "My mom's goes, 'just another bad thing is going to come out about Muslims'...You know, it's just rough being Muslim." Fathima has moved around the US says that from her experience, that it is much easier to be Muslim on the "coasts" of the US, rather than the "internal, red" states. Fathima considers geography significant to her perception of discrimination. She feels safer away from areas that she sees as "red." The coasts are places where she has not only personally experienced less discrimination, but they have also been represented through news media as having fewer instances of discrimination because diversity is more likely.

Nazeem, who also identifies as Muslim and more broadly desi, described the frustrations with post-Trump election America and says that it affects her in daily life. She considers geographical location significant to her experiences as well. She grew up in Texas and lived in Indiana for a while. She would have been much more concerned living in states like Indiana or Texas, but now that she is in Boston, she feels a bit better. "I'm in a very happy bubble in Boston right now with the kinds of opinions around. It is a bit of an echochamber which is a problem, but at least it is a safe space." She says that there are others who think like her and she feels that she does not have to constantly worry about not being white like she did in other states. But, for Nazeem, even though she lives in what she considers a "bubble", she is quite concerned about the Trump election rhetoric. She says that the damage of the Trump election is irreversible. 
What bothers me is that the damage is done. There is no going back. One the one hand, this guy is president and you figured out which of your friends is racist. It is like they aren't bad people, but they didn't give enough of a fuck about other people's problems. Like if you are struggling to put food on the table, you don't give a fuck. Then, there are people who are doing well, who are like, this is better for my bottom line. People you work with every day, bosses, people in power, and that is scary. They are not going away. People say, at least they are honest. I'm like no, keep that shit hidden. It was better that way because at least the stigma was there to keep you silent. But now, well, if the President was ok with it so am I. I'd rather you be silent than assault or harass someone. Now they feel like they are right.

She says that she does worry about how it will affect her family, friends, and even situations at work. She finds that she is always wondering what someone is thinking, if they support Trump, or if they are secretly racist. Though, this depends on the situation and context. Her geographic location is safe for the moment, but she is not sure what the future holds and that concerns her. I found that many participants expressed concern and caution in public life, works spaces, and other aspects of their lives. They described uncertainty in that they did not know what to expect from employers, co-workers, other people around them. Many participants went into detail about the emotionally-laden aspect of the Trump election. But some participants were much for responsive than others. Some said that they worried, but they did not really want to discuss it. It created too much emotion and they were still working through their feelings and reactions. Many mentioned that they 
continuously worried about what people, generally white, were thinking about them. This is often when they began to use Indian, South Asian, or other broader terms along with brown. Their concerns before about being "other" or "different" were heightened significantly.

Vimala and I talked further about how the election was still difficult to process and what it meant for South Asians living in the United States. We discussed what it really meant to be American and the idea that while anyone who was born in the US is American, the dominant representation of American is visibly, audibly and culturally white (though there are some changes happening). Many participants mentioned that Indians are no longer associated with being white and that concerned them. Some participants mentioned that before 9/11, people did not think of Indians as brown like they do now, and Trump is making it worse. The Trump administration further emphasizes these dominant representations of whiteness. Though academia has addressed issues of whiteness in Trump's America, they have also surfaced in popular media. Articles like Toni Morrison's (2016) “Making America White Again," or Ta-Nehisi Coates (2017), "Donald Trump is the First White President" have been shared, referenced, and discussed on social media, news, podcasts, and other outlets. In fact, many participants, while not directly referencing articles, brought up these sentiments. The phrase "Make America White Again" was significant to many participants who feared that they could no longer be American because they were not white. But, whiteness is not just a skin color and while being Indian is associated with being brown through a variety of senses, particularly sound, Indians can also be associated with being white as I will discuss in Chapter 8. 
The rise of Islamophobia and implication of brownness

For those who lived in the US before and after 9/11, many, like Vimala, mentioned that other Americans, often white, started to treat them differently. Participants reacted in a variety of ways. Participants like Nazeem said that at first, she sympathized with people's reactions, but slowly became aware of the implications of being brown and Muslim in the US. She especially now draws parallels between post-9/11 and post-Trump election.

The week of 9/11 I had a teacher pull me aside and say if someone says something, you tell me. I was like ok, thinking it wouldn't be a problem... I understood the fear response from 2001-2005. I kind of understood that. Now, I just don't have sympathy for that anymore.

After 9/11, many participants became aware that they were different, that they were not white, or they became conflated with the 'Middle East.' Even though many from the 'Middle East' are considered white according to the census, 'Middle Eastern' is often associated with non-whiteness (Naber, 2012). Participants often attributed their differences to being Indian. This happened in many ways. For Lakshmi, she said she was always aware that she was different. "I know if you live in some other country you kind of have to adapt to belong with them. First of all, in (University town name) ${ }^{34}$, I didn't feel like a foreigner. There is a lot of diversity." But, she said that the Trump election and 9/11 changed the way that

34 I omitted this town name because it was a bit too specific and might have given away the identity of the participant. 
people acted around her and reinforced that she and her children, were not really American.

Even now, I feel like people do talk with a different attitude. Was I treated bad? People assume you don't speak English and you try to understand them. Anyway, we are always treated as second-class citizens. That, you feel. You have to work 10 times more and that I still worry about that for my own children even though they were born here. I don't think they will be treated equally like the Americans.

She makes a distinction between white Americans and Indian Americans. For Lakshmi, and many others, they did not feel completely American and felt that they white Americans will never really accept them. Lakshmi attributes this to a variety of factors. Accent is also a significant part of this experience for her. She was marked as "other" or different precisely because she spoke with an "Indian" accent. She said that people notice accent right away and you cannot hide it. For some, skin color was an issue, others, accent and language were also significant, and for many, both. All of these markers of non-whiteness were often linked. For example, Prisha described many incidents, but one was quite important for her.

What I can remember is just as soon after 9/11, I was at a nursey and I got the products I wanted and went to counter. Two people at the counter, they were both guys at the other end. There was this lady wearing dark sunglasses and they kept talking to her. After they were done, they still didn't come to me. This lady puts her hand on counter and stares at me. I ignored it and these guys kept laughing and 
staring. This is the last time I went to the nursey. I realized she didn't know who I was, but my darker skin color bothered them.

Prisha, like many others, connects this to accent and language. While this is not necessarily a direct result of 9/11, many distinguished heightened security climate of a post-9/11 United States and rising anti-immigration sentiments. They noticed that Indians become much more associated with brownness - through accent as well. Prisha said that she also experienced discrimination with accent, before and after 9/11.

When I came early on when I spoke early I'm sure I had accent. I spoke quite fluently. (I'm sure I still do, but it is better). Here is what I thought. People predecide that they are not going to understand you. They don't listen or stop listening. I can speak, I'm sure you can understand me. So, they made me feel uncomfortable, bad... Even my own advisor, she would correct me. 'Hey, you need to make sure you pronounce these names correctly because it is disrespectful', yet they would mispronounce my name all the time.

Prisha, describes how attention to accent makes her uncomfortable, sometimes more uncomfortable than skin color. She says that once someone brings attention to her accent, it is hard not to be aware of it. "As soon as they put you on the spot, your accent thickens. You get more uncomfortable. I would consciously make an effort to relax when I realized what was happening." 
As Kanngiser (2012) and Dave (2013) have discussed, accent is significant to the ways in which people perceive others or experience discrimination. As Dave (2013) suggests this often comes from how popular mass media represents accent. For example, "Indian" accents are homogenized, but also branded as "other" or as deviating from a standard norm, which is often associated with whiteness in the United States. As Kanngiser (2012) argues, accent is also important to the way people experience daily life. People perceive speech tones to be indicative of certain characteristics, like how Obama's speech tones during his election influenced the way that many people viewed him, either as black or white (Kanngiser, 2012). Beyond these implications, accent was significant to how some participants identified discrimination. For example, as mentioned before, in many cases, participants were more likely to acknowledge discrimination or identify discrimination when discussing accent or language. For many participants, accent was significant to how they experienced discrimination. In some cases, participants only acknowledged discrimination through accent or language.

For example, Punniya, initially said that she never experienced any issues in the US where she felt people discriminated against her. I then asked her if she had any experiences like this with accent or language. She responded:

I'm truly worried about my English. There are words I can't catch. Or if I can't understand or I can't answer, what to do? This is very bad thinking, but it is the main reason I don't go out. Even if they are making fun of me, I can't understand. If they make fun of me, I can't understand it. Maybe they aren't in in a mean way, but they are still joking...Sometimes I face issues, but I try to forget about them. 
She says that people have made fun of her for her accent before and she says she understands because back in India, she, and others sometimes for fun, would joke about the way others speak Tamil. This makes her so afraid to go outside. She said she goes to the library and volunteers at a hospital and there, she does not worry about people teasing her. These places are more diverse, she describes. It is only when she goes outside that sphere that she has had those experiences and those are the places that she fears.

There are many others who initially said that they did not experience discrimination often discussed accent as being an issue. Vijaya says that she does not go out much, but when she does, people sometimes cannot understand the way that she speaks. She came to the US in the last 5 years so she says she cannot speak much to the post-9/11 or postTrump election climate. I found that those living in the US longer were more likely to describe discrimination than those living here for a shorter amount of time.

Ruth, who came to the US in 1982, says her experiences with discrimination also involve accent. In her church, she says she doesn't feel any discrimination, but, in public, she says she felt it especially after $9 / 11$ and even after the Trump election. In church, she says the people who know her, know her culture. She says the people that do not know her, she doesn't talk to them anyway. While the looks and the stares come often, she says that accent and language have been a large part of her experience with discrimination. She does notice that people in public still sometimes pretend that they don't understand her, and she noticed this especially after $9 / 11$. She said it used to be worse, but she still sees it. "They switch off their ears. I can understand them, they should understand me. If they see a different color, they think you don't speak English." For Ruth, and a few other participants, 
accent and looks often intersect. It is not that she just looks brown, it is also that she sounds brown and vice versa.

Many participants mentioned that if you look white and have an accent that it is much different than if you are brown and have an accent. Even second-generation participants that I interviewed, who grew up in the US described instances where white Americans assumed that they had an accent or had commented on how "they speak English so well." Vimala says that it happens to her all the time. "I've gotten that, you speak English very well or you are very articulate. Why? And relative to what? I've been the recipient of that 'compliment."' Nazeem also experienced this. She says that her experiences with accent reinforce her American identity and she becomes more assertive. She said working retail in New York, she has run into a lot of issues with accent:

'Oh I really like your accent.' I'm like what accent? She (the person checking out) assumed that because I'm not American-looking, she assumed that I didn't ...(pause) she clearly attributed something to me that wasn't there. Also, the 'where you from, no where you really from?' Working at retail people feel like they need to ask. I'm usually like America. That is when I really strongly identify as American. They are like really, no, where you really from, I'm like no really, fuck off.

Both Vimala and Nazeem described events in detail, but many others, especially other second-generation participants said that they had experienced this as well. Vimala said that she also ran into issues when she was with her parents. 
I experienced it through my parents. My parents still have their Indian accent. We've gone shopping together. I'll repeat what they are saying to the attendant. There was this one time this person made my mother repeat herself over and over and over again. This person had a smirk on their face. It was one of those times I called them out on it. Please stop. I've experienced through them and I don't really have an accent.

Anusha also described this, but while she was concerned with accent, she was much more concerned when her parents spoke Tamil in public. "When we are traveling when my parents are talking in Tamil, I get nervous. We are very brown and no one can miss it, but the way we sound and dress brings attention to us." While participants recognized the effects and shift in discourse post-9/11, it was very much connected to ideas of brownness and Islamophobia. 9/11 was a catalyst for heightened anti-immigrant and anti-muslim discourses that manifest in participants' everyday lives through brownness. Brownness was also connected to sound within these examples. Accent, specifically, became another marker of difference that identified participants as brown and non-white. Again, while the discussion of brownness is relevant beyond 9/11, many participants described that Indians became more associated with brownness after 9/11. Prior to 9/11, many participants thought that Indians were not as closely associated with being brown.

\section{Orientalism and othering}

Many participants described that they did not experience as much misrecognition or stereotyping associated with Islamophobia, but instead with persistent stereotypes of 
poverty, backwardness, or "third-world" lack of development. These ideas were very much a reflection of Orientalism. But, these stereotypes persist today and for Indians, now often coincide with ideas of spiritual exoticism - the idea that India is exotic because it is spiritual, often idolized in the West through yoga, Hindu symbols used on or as clothing, and other forms of cultural appropriation (Bandyopadhyay \& Morais, 2005; Jain, 2014). So, although some participants described these as pre-9/11 stereotypes, they are relevant and continue even in a post-Trump election era. Many described how people think of India as poor or backward or as a place for spiritual tourism. Ruth described a few instances when Americans asked her, what she refers to as "stupid" questions. "They asked, did you change your name after coming here, Ruth? No. I've been christened with that name. They said, we didn't know there are Christians in India." She speaks in a sarcastic, witty tone as she describes these incidents. She finds them to be amusing and frustrating at the same time. She then lists a series of questions.

Do you have snakes on the road? We hear that animals are on the road. Oh, you used to have electricity? I said yea. Because they have never moved out of this country that's why... Here, people are so ignorant. In India, even in the village people are smart. They won't have gone to school, but they are smart. That is one thing I found out. Ask them about US and they will tell you everything. Ask these people and they don't even know where India is.

Ruth is not the only one to describe these sentiments. Many linked stereotypes of India to othering and discrimination. Bimal, who teaches at a high school in the Cleveland 
area says that stereotypes of India are persistent in education. This, in turn, fuels othering and discrimination in the US, because it creates single stories of people and places and creates a culture that cannot recognize nuance. He says that schools in the US teach people that India is a place of poverty or backwardness. He said that many do not know that India is on the forefront of development or that it has malls or things that you might only see in the "Western" world.

During my participant observation, I also had conversations with a few people on the way that Americans see India. During a 2017 Deepavali celebration, the table discussed how people in the US tend to only see the negative aspects of India - that it is dirty, poor, backward, or does not have any technology or luxuries. A few members of the table were high school teachers in Northeast Ohio. They described concern about what Americans are taught about countries like India. They felt high schools and high school education reinforces many stereotypes about India and other developing countries. ${ }^{35}$ This is turn causes many Americans to not understand or recognize nuance, fueling misrecognition and even hate crimes.

Some participants even described that they were often mistaken for Native Americans or at least, people would joke to them about it. Arvind, for example said that when he first came to the US in the 1970s, people made jokes that he was Native American. “They were calling me chief. They used to say, 'A good Indian is dead Indian.'” Arvind said though, that he took it as a joke and did not actually worry about someone killing him, like

35 While this is not directly related to my research, I did serve as a reader on the AP Human Geography exam. I have argued that the AP Exam does indeed reinforce and reward representations of "third-world" countries in this way. 
he worries about now, post-Trump election. Like many others, he says that he was "welcomed with open-arms" when he first came to the US. Yet, he also describes incidents like this one.

Others also experienced these stereotypes growing up in the US, which many viewed as catalysts to discrimination. Some even described stereotyping as a form of discrimination. Often, they had different views, depending on circumstances. Some did not see discrimination as an issue until recently, like Arvind, and others stressed that it defined their identities. Regarding discrimination, Fathima and Suhail recalled different experiences while growing up in the US. Suhail said that he never thought he experienced much othering or discrimination.

I almost wonder if it is the way that I am. I feel that my Americanism is very genuine. Like, oh ok that guy is on our side. Whereas some people aren't pro American and other people can tell. I worry about other people though.

He later says that he wonders if it has happened and he just has not realized it. Even when he went to medical school, he said that at least half of the students were Indian, so he did not really pay attention.

Suhail describes being genuine American. I heard this from a few other participants too. Though the definition varied, being American, in many ways, was assimilating to dominant, white culture (Bhabha, 1994; Dyer, 2010). Those who are products of the legacy of colonialism, Indians or others, mimic the colonizer in behavior, attempting to gain the same power as the colonizer or the dominant group. This is evident with US politicians like 
Nikki Haley and Bobby Jindal, or conservative spokespersons like Dinesh D’Souza or Ramesh Ponnuru who mimic dominant white, Christian, American values (Chand and Tung, 2014). Furthermore, many US Hindu nationalist associations are some of the biggest supporters of Donald Trump and promoting dominant white culture (Peters, 2016). This is in part an effort to "assimilate" or to keep from being marked as different or "other" (Bhatia, 2007) and as Bhabha (1994) might argue, it is a way to achieve some form of colonial power. I discuss the concept of mimicry further in Chapter 7 during my discussion of discrimination within Indian communities.

Unlike Suhail, Fathima, on the other hand, who strongly identified as Tamil, not Indian, when first describing identity, recalled many experiences with discrimination because she was "Indian." She also connected these to geographic location. She describes that her views were shaped by the fact that she was in school in a rural area, while Suhail was not. She says because she is 5 years older than her brother, by the time he was in elementary school, they had already moved to a more diverse neighborhood. "For him, his memories of that part of our life were very few." She said that her parents desperately tried to get them in a different school system because they were worried about the interactions with the rural neighborhood. Fathima recalls a few comments:

I was in like second or third grade, we lived in a more rural town...Someone being like, oh my god they are so poor that their mom wears sheets every day. I was like oh my god, you guys just have no idea. And in fact, I think we were probably the wealthiest family in the block there, and I think people just didn't get it. Kids used terms that they didn't even know what it meant. Derogatory terms like "tar baby" 
and stuff. I don't even think I knew what it meant at the time...I had a couple things like that that I'll never forget.

They lived in the same area, yet because they were 5 years apart and went to different school districts not even 20 minutes apart, they had vastly different experiences. Suhail attributes this to personality and how each perceived various interactions and situations, while Fathima says that it is because of the difference in the geographic location.

A few other siblings that I talked to had similar discussions. But these discussions were not just related to geographic area. They also revolved around growing up in pre-and post-9/11 periods and markers of difference that made them non-white as well a nonstereotypical Indian. Vimala and Sakhti were siblings about 10 years apart and described both similar and different experiences with discrimination. Both acknowledged that their experiences were different in relation to 9/11 and when they grew up. Sakhti said that while she experienced discrimination, Vimala might have experienced more because she was still in school after 9/11. Vimala, says that though 9/11 was significant, said that she experienced a lot of racism with pre-existing stereotypes and being one of the only minorities in school.

Growing up in a predominately white, middle class neighborhood, I felt like I didn't belong. I was teased a lot. I was very aloof, so didn't fit that stereotypical Indians are smart. I was in a whimsical state where I didn't know what was going on. We prayed a lot. My parents would put pottu on our foreheads and I tried to wipe it off .... I can 
still be very, very self-aware. I can tell I'm very different still. When I'm in predominately white spaces I can feel uncomfortable.

Markers of brownness were significant to Vimala's experience and to many others. She said that students would make fun of her for her curly hair, which became a marker of difference. Like many other participants, her body was constantly policed. Sakhti described the same experiences, but in more detail.

Out of the four Indian people (in her school), I had uncontrollable curly hair. I was always trying to manage my hair. My mom would put coconut oil on it. It would just look greasy...I was sitting at lunch and a girl tried to wipe my hair down with a dirty sponge. I lost it. One time in high school this kid took a lighter to my hair.

Both sisters described that the stereotypical view of Indians is that they have straight, beautiful hair. Neither of them fit this stereotype and in a mostly-white school, this was a marker of difference. Vimala also said that another marker of difference is also in the way that white Americans sexualize "Asian" women, especially Indian women. She said feels uncomfortable with the type of sexualization that grown, older, white men have of Asian women in general. The fact that they describe her as exotic or comment about how she is somehow more sexual because she is not white. She is frustrated that when she confronts someone on issues like this, they respond with “It's a compliment so why are you taking it that way?" Vimala says they do not understand why it is offensive and describe her as a being too overreacting or angry. 
Anusha also describes this discomfort and discrimination with being marked as Indian or brown, which she used interchangeably, in the US. While she experienced racism for being brown and Indian, she also experienced it in the form of sexualization. "I was being exoticized and overtly sexualized because I was brown." For Anusha, being brown affected many aspects of her life.

I've definitely gotten similar harassment for being queer, I don't present as gender non-conforming, but when I'm out with a woman or something, I feel pretty strongly that because I'm like darker-skinned, and you don't pass for anything else, it definitely impacts my life. I'm happy being brown, but I definitely think in professional settings, it has an impact in asking for promotion or new opportunities or things like that.

In other words, being Indian or brown, impacts almost all aspects of her life. In many ways, she cannot forget her identity or choose not to identify as non-Indian because she is brown. Aural discrimination and stereotypes were significant for participants as well and often intertwined with their experiences of brownness. Earlier, Vimala had described how people told her that she spoke English well or even perceive that she was supposed to have some kind of accent. But, she also he described the kind of questions that make her uncomfortable often related to the way that she looked as well as the perceived way in which she spoke. "Where are you from? New Jersey. No, where are you really from? Just being in places and people looking at me like I didn't belong. Why are you here?" 
Vimala, among others described how accent was significant to their experiences of discrimination and became another marker of difference or brownness. But, for some participants like Matthew, accent, was key to how he faced discrimination both before and after $9 / 11$. While the 2016 election was a significant source of concern for Matthew, he experienced discrimination much earlier. Growing up in Alabama pre-9/11, Matthew says he has experienced a lot of discrimination focused on his accent. "I got made fun of for my Indian accent. And the way I dressed. Quite often on multiple occasions." He recalls a specific instance in school that led them to change their last name, "I thought it was weird, on an 8th grade science test the bonus question was how do you spell (Matthew)'s last name? We changed our last name because of that."

For Matthew, it was not just the way he spoke, but also the way that his name sounded. The pronunciation of his name and the way that people heard it was significant. He said that if you have a more white or American sounding last name, you are less likely to experience discrimination in job hiring, schools, among other things. Though this incident drew attention to the last name, it was not the only reason that they changed it. The last name he has now is no longer "Indian", but sounds quite American, like a Smith, or Jones. He said this made it easier for them to find employment, for people to pronounce the name easier, and in the end, be viewed as more "American." In other words, it made them more culturally and aurally white. He mentions that his teacher was trying to make other students more culturally aware, but she still brought attention to how he and his family were different.

For Matthew, accent was quite significant to many of his experiences with discrimination and being "othered" His Indian accent caused him to experience 
discrimination on multiple occasions. One incident, he recalls in detail, "When I first moved here I had a strong Indian accent... I remember coming out of a movie theater, the people in front of me faked an Indian accent and I snapped back in a fake southern accent which they got the point." Matthew, when he first came to the US, described that being Southern American and Indian were often contradictory. Yet, later in life, he said that he can sometimes inhabit both of those identities. He says that even though he experienced discrimination based on accent, he is also guilty of it as well. "Certain sound-based discrimination, like I'm guilty of that too. If I call into a call center, and it's another accent, I also find it difficult. I'm not completely immune to discriminating against someone."

\section{Web of discrimination}

For participants, discrimination in the US was incredibly complex. Participants often scaled up to describe discrimination, but also began using some scales like Indian and Tamil simultaneously. Participants, like Matthew, used regional scale even to describe American identities, pointing to American identities like Southern, which he described as being in direct opposition to identities like Indian or even Tamil. Yet, geographical location was also important to his experiences and the way that he framed his identities. While participants weaved in and out of various scalar identities, they also used them simultaneously and in relation to specific geographical contexts in what Kaplan (2018) describes as "multi-scalar in a geographically-complex world."

For example, Matthew identified as Indian in the context of the marginalization he felt in Alabama, but when he was in other parts of the US, he identified as Southern, specifically because of the marginalization he felt based on his Southern accent. Matthew 
said that he came to the US when he was 9, so he initially felt discriminated for his Indian accent and thus identified as Indian in those circumstances. But, he was able to change his Indian accent and developed a Southern accent. Subsequently, he experienced issues with his Southern accent. He says that it was noticeable when he changed geographical location. He moved out of the South to Northern states. People would point out that he sounded funny or Southern. He then described his identity as Southern. He says that his oldest brother still has an Indian accent, but he describes his accent and his youngest brother's accent as "super Southern." He finds it fascinating that they all have different accents and that creates different world views and experiences for each of them. For Matthew, accent and geographical location informed many of his experiences with discrimination.

Matthew is not the only participant to experience othering based on his American, Southern accent. Nazeem also experienced this layer of othering along with her other identities like being Muslim, desi, Pakistani, or Tamil. She describes how this affects the way that she experiences all her identities. Geographical location also influenced her experiences. Especially, when she moved from Texas to Indiana.

So, I had at the time, not really a drawl, but I'm from Texas, like y'all. So, I would get made fun of a bit for the drawl. There are people who had a bit of discrimination (figured a bit of minority - for sure in Indiana), when I was like 10. ‘Oh, you sound funny.' I feel like had I been there post-9/11, it would have been worse, so I'm glad I didn't live there post-9/11. It was seen as like a country bumpkin type thing. That was because of my Southern accent. 
Both Matthew and Nazeem had experienced othering, which they thought of as a type of discrimination, based on their Southern accents and also in the South for either real or perceived "Indian" accents. Jansson (2010) argues that Southern identities experience Orientalism because they are deemed not part of the norm or inferior to the rest of the US culturally, and therefore become associated with regional resistance. What Jansson (2010) also acknowledges is that these identities of Southern are primarily identified with being white. For Nazeem and Matthew, they were identified as Southern based on their accents, but also clearly identified the discrimination that they felt while being in the South for not being white both visually and aurally. In many ways, they separated themselves from the Southern identity, but also experienced being part of it through accent.

The ways in which participants experienced discrimination or othering was much more in part for being non-white but also affected by accents associated with whiteness. For some, experiences with discrimination were more prevalent in other places that they had lived prior and they often compared these experiences to what they faced in the United States. For example, Santhya said that while she worries about discrimination in the US for her children, she had experienced more discrimination in Europe. She says the US is a little more open and she feels less isolated. She says despite what is happening politically in America, she feels more able to be a part of the politics.

"Definitely, I think that the current climate here has made me more sensitive to that happening to my children. They are light skinned, but don't look like a light-skinned American." She says it is prominent in the US, but not like what she felt in Europe. Here, she is more concerned for her children than herself. In Europe, more specifically Switzerland, she had a difficult time. "When I was growing up, I don't think there was a single day that I 
ever didn't feel marginalized." She describes a few instances in detail. “There was a stationary store, the owner was a horrible guy, he really made it clear that he didn't like me or any boy or girl of color. He treated me differently than a white or Western girl. Even a few times, he charged me more."

For Santhya, she experienced both visual and aural discrimination. It was not just the way that she looked, but also the way that she talked. "There was a time in 2 nd grade where people didn't sit next to me because they said I was dipped in poo. I remember being made fun of for looking different or that my parents sounded funny when I (and they) talked." For Santhya, it was both issues with accent as well as language. She said she remembers being embarrassed when her mom tried to speak to her in Tamil in public or even around non-Tamil speakers. People would notice and make comments. It was another marker that she was different and "other."

In the US, she says that she did not directly encounter that level of discrimination. But, she said that her children will have very different experiences in the US than she had. She says the same was true for her experiences compared to those of her parents when she was in Europe and regarding the first-generation immigrant experience. But, she says that even though she is a first-generation immigrant in the US, she is not a first-generation to what she describes as "Western" culture. For her parents, the difference was in that they did not want to draw attention to themselves. She says she sees that her parents are still affected and she notices through discussions and body language. She says that they have an attitude of "we know we are not supposed to be here, but we aren't trying to be bad." This is similar to what Suhail describes as being more of a "genuine" American. The idea that they are trying to assimilate, not go against any norms, or trying to fit in to "whiteness." 
Many others, not just Santhya, described that they had experienced more discrimination in other places. Murugan said that he experienced more discrimination, not with skin color, but more with the Tamil language, when he lived in Malaysia. His grandparents were from Tamil Nadu and he grew up in Malaysia before coming to the US. He says that he experienced it a lot when he was in primary school and high school but began to notice it more as he got older. He compares this to what he has experienced in the US.

People are like “oh you speak Tamil” and I wouldn't say I divide myself, people divide each other. Maybe it happens still, but I don't pay much attention anymore. I got immune to that. I wouldn't know. I mind my own business. First few times, you feel like "Oh." After several times though, you get used to it. Basically, you're immune to it. It is better in US than in Malaysia. Here, it is not right in front of my face. I don't know what happens behind my back.

Many said that discrimination may happen and they just do not know about it because it happens privately in the US. Many are concerned with the current political climate because they worry that this type of discrimination would become more culturally and openly acceptable. Even those who described that this discrimination was worse in other places, also acknowledge that they have faced some discrimination in the US as well. Santhya, for instance, recalls a few occasions where she felt discriminated against in the US. In these experiences, language or accent were key components. 
'How do you know English so well?' I've encountered it a few times in the US. And also, when I was living in New Jersey and I had joined a French speakers' group. In that group one of the women asked me where I learned French. She had the assumption that my husband must be white and the only reason I knew French was because my husband taught me. In her mind, it was that I had been brought by a white husband and that is why I spoke French. I wouldn't necessarily be myself without being rescued from a life of misery from a developing country.

Accent and language were significant to many participant's experiences with discrimination or othering. Many scholars have written on the significance of visual discrimination. But, other senses are significant to experiences of discrimination. In some cases, smell and other senses were also significant, much like Haldrup et. al (2006) have theorized. Many participants also discussed how food and smell were significant to their experiences growing up, especially because others had marked it as different or not part of the norm.

Kumaran, for instance, described how his peers commented on smell and food. “'Why do you smell like curry?' The way that your house smells is weird." Quite a few participants had described this curry smell or the weird smell that related to "Indian" food. But, they did not just mention smells and described foods as different. He said that one time, kids even asked him if he ate monkey brains because they had seen Indiana Jones and the Temple of Doom and this was their only exposure to "Indians."

For many participants, discrimination was a multi-sense experience that included sound and at times, even smell. One participant even described that they experienced being 
othered through the idea that someone thought Indian food "tastes" bad. Although, other participants mentioned that many Americans like them because they like the taste of "Indian" food. The stereotypes and homogenization of "Indian" food also became problematic for many participants. While I did not ask about smells, tastes, or other senses specifically, they came up often, reinforcing that discrimination and othering are a multisense experience. I will discuss the implications of investigating additional senses in Chapter 8, as well as their relevance for future research.

\section{Context and US Discrimination}

Overall, discrimination was a multi-sense experience which stems in great part from visual and accent markers of difference. Sometimes, it was informed by smell and even taste. Geographic location was also incredibly significant. Participants identified that they experienced more encounters with discrimination in geographically specific areas - often described as non-diverse, rural, "red," and small-town. In many ways, these shaped how participants viewed identities and how they addressed discrimination.

Participants still at times, used hierarchical scales to define their identities, but also described them as more blurred, hybrid, and at times multiple. Most participants identified with larger scales like Indian when describing discrimination in the United States, even if they had previously identified with smaller scales like Tamil or more local scales like from Tiruchirappalli (Trichy). Even those who did not identify as Indian when discussing identity or who identified globally, scaled down to identify as Indian when describing othering or discrimination. In general, participants identified more strongly as Indian or even American when describing their othering or discrimination experiences, even if they 
were misrecognized as non-Indian. A case in point being when Maari described that he was misrecognized, he acknowledged that this was because he was Indian and had visual or aural cues that others deemed as 'Middle Eastern.' Even when discussing identity, some participants did not directly identify as Indian, Tamil, or American, but did so when describing personal experiences with discrimination. For example, when Lakshmi described her fears for her children, she continuously referred to Indian, Tamil, and American, using them in different contexts.

Even though they are all Americans, they look Indian, so immediately they won't be treated exactly like another American person. That is why I tell them they should keep up our culture as an Indian, not just a Tamil, but at the same time not change stand up and be proud of our background. I want my children to be proud of being Indian and American and myself too.

Tamil is referred to as a subset and regional identity, one that needs to be minimized to identify with a broader, national scale of Indian. Initially, she describes Indian and American as opposed, but suggests that ideally, they should co-exist. Many participants shifted between identities using them in specific contexts. The discrimination that they faced throughout their lives and especially in the United States for being non-white through a variety of senses, affected the ways and moments in which they identified with specific identities or scales. Discrimination was connected to scale. For example, participants identified as Indian in the context of discrimination faced in the US, even if they had previously identified with smaller scales like Tamil. Yet, as I will demonstrate in the next 
section, when facing discrimination in the Indian community, they again began conceptualizing identity through smaller scales.

It was also important for many participants to try to exist in hybrid spaces with multiple identities, embracing multiplicity for solidarity. For these participants, there was something incredibly important about brownness and communities of color sticking together. This was precisely because the pervasive cultural whiteness of American identity became incredibly problematic, both in the US and within Indian communities. Nazeem describes the importance of marginalized communities coming together, and how it is crucial to her identity:

For me, context is everything in my identity. The first issue was $9 / 11$, how to balance Muslim, desi, etc. The next was having to deal with a very open, public display of the country you thought you were part of now doesn't want you here. It doesn't matter that you born here or lived here, or spent your whole life here, you will never be part of them. That is very disheartening. In a funny way, my mom was right, you will never be one of them no matter what you do, so why try? It is times like this where you need to blend in more or lean on your cultural heritage and you are like I'm this and American, what of it? That is a lot of what our generation is like. I'm gonna fight for your rights as hard as I'm going to fight for mine. I've noticed a lot of marginalized communities coming together because they (the current political leadership) don't like anyone who is different from the Anglo American. Then there are the others who have justify it and are like we are in a post-racial blah blah. 
Nazeem brings up the implications of brownness and how it has been defined post-9/11 and post-Trump election, which leads to her discussion of the importance of marginalized communities coming together. For quite a few participants, this was important. Yet, many acknowledged that Indian American communities have internal discrimination issues and often try to distance themselves from other communities of color. I continue this discussion in Chapter 7, while discussing marginalization within Indian and Indian Tamil communities. 


\section{Chapter 7: Discrimination, "Othering," and Indian Identity}

This chapter builds on Chapter 6's focus on discrimination by addressing discrimination within Indian communities in the US. Participants described experiences with discrimination both with non-Indian communities and within Indian communities. As mentioned in Chapter 6, when discussing discrimination, participants tended to identify through national or supranational scales like Indian, desi, or South Asian. Yet, they became more nuanced and referred to regional and even more local scales in their descriptions of discrimination within Indian communities. Thus, while the focus of this chapter is discrimination, both scale and hybridity appear throughout. Finally, sound was important to discrimination and informed many participants' experiences.

Like in the last chapter, discrimination had different meanings for each participant. To reiterate - for some, discrimination was a hate crime or an act of physical violence. For others, discrimination was simply active - they were denied opportunities because of their identities. Yet, some participants described it as a form of "othering"- they were looked at, treated differently, spoken to in derogatory ways, or scrutinized because of specific identities. "Othering" was also connected to microaggressions, whereby subtle and pervasive forms of racism create numerous psychological effects on those who experience these subtle forms of discrimination and even contributed to more active forms of discrimination (Joshi, McCutcheon, \& Sweet, 2015). Many participants said they experienced discrimination and "othering" within Indian communities. Those who identified using large scales like Indian, South Asian, or desi in the last chapter, began to 
use smaller scales like Tamil, when describing experiences with other Indians. Many participants said that they felt marginalized based on being Tamil in a larger Indian community. Participants also identified more locally, especially when describing that they felt marginalized within a larger Tamil community because they were from certain regions of Tamil Nadu and thus, did not fit specific normative categories. They relied on small-scale identities to describe discrimination and "othering" relating to region. Some discussed how North Indians marginalize South Indians or how the rest of India views Tamils negatively.

Hybridity was also useful to understand participant identities in a post-colonial world. Colonial binaries and categories were reinforced throughout their experiences. For example, some participants described that others criticized them for being too Tamil, too Indian, or too American. In other words, even though at times, though some described their identity as hybrid, participants said that they still felt that identities were often represented as categories and binaries. These categories and binaries became a bit more complex when participants shifted from discussing discrimination and othering in US communities to Indian and Tamil communities, yet they were all in some way connected to whiteness, which I discuss in more detail in this chapter. Some also described the colonial legacy that marginalized people from the South, deeming them as inferior or backward, and how that persisted and was mimicked even today. Finally, some participants described the relationship between Indian communities and other minorities as being problematic. Participants mentioned discrimination and "othering" against other minority communities, which was also connected to whiteness. The discussion of internal discrimination, in many ways, fits is very much related to mimicry. 
In this chapter, I elaborate on Chapter 6's discussion of the importance of mimicry and how it fits into narratives of Indian whiteness. As mentioned in Chapter 3, Indians often strive to be white, yet people often mistake them as Middle Eastern, which according to the census is white, but effectively non-white in Islamophobic societies (Hopkins et. al, 2017) or black. The authors suggest that these tensions between white and black are mimicked between dark-skinned Indians and light-skinned Indians, often embodied in North vs. South, something I elaborate on further in this chapter. In other words, antiblackness and brownness are issues within Indian communities, which contribute to internal discrimination of dark-skinned Indians (Koshy, 1998). Whiteness or what Bhatia (2007) refers to as "brown privilege" are significant issues within Indian communities. Brown privilege is the idea that a group experiences some privileges of whiteness but does not necessarily reap all of the benefits of being white (Bhatia, 2007). 'Anti-blackness', a term that Sridaran (2017) uses, describes racism toward other minority, often black communities, but is also connected to discussions on darkness and lightness within Indian communities.

Building from my discussion of Critical Race Theory in Chapter 2, I reiterate that whiteness is not just indicative of skin color. Dark skin is indeed a marker of difference, but I argue that sound and other senses are also incredibly important to discrimination. Scholars have linked language, accent, music, smell, and taste to how dominant groups in Western, often white, societies, construct "otherness." (Haldrup et al., 2006; Simonsen, 2010; Dave, 2013). As such, groups like Indian Americans, even though they have experienced discrimination based on skin color in post-9/11 climates, have also been linked to whiteness in many other ways through economic standing, profession, and other 
factors (Bhatia, 2007; Chand \& Tung, 2014; Inwood \& Bonds, 2016; Safran et al., 2008). In Chapter 7, I described how many participants said that they did not experience discrimination based on skin color ${ }^{36}$, yet described it based on language and accent. In other words, participants were being monitored by others and monitored themselves through sound. I continue this discussion throughout the next four sections.

The first section of this chapter outlines discrimination of Tamils in the Indian diaspora. The second section describes discrimination within the Tamil community in the US. The third examines the problem of whiteness or Bhatia's (2007) "brown privilege" within Indian communities in the US. Finally, the closing section addresses nuances of discrimination in Indian communities.

Whiteness and marginalization of Tamils in the Indian Diaspora

Many participants described that they felt some discrimination or "othering" as Tamil in the broader Indian community. Again, discrimination varied from intentional/overt discrimination to feelings of being othered or experiencing microaggressions. Some linked discrimination to skin color and others linked it to language and music. Koshy (1998) and Bhatia (2007) describe these tensions between North and South India as also being related to divides between Aryan, from North India, and Dravidian, from South India. "Aryan" is often described as being the true "white," superior race, while Dravidian is considered the darker, less superior race (Jacob, 2009; Koshy, 1998). These divisions are more than skin color and involve language and music as well

\footnotetext{
36 Even though they might have experienced discrimination based on skin color, they perceived that they did not.
} 
(Viswanathan and Allen, 2004; Jacob, 2009). Divisions between Aryan and Dravidian, reinforced by British colonialism, are also linked to languages as Aryan and Dravidian are also used to describe language categories (Bate, 2009; Jacob, 2009; Schwartzberg \& Bajpai, 1992). Aryan languages come from the North and Dravidian languages come from the South.

Tamil Nadu's history was significant for many participants (see Chapter 6), and also sometimes connected to debates regarding Aryan and Dravidian. The debate between whether to identify nationally as Indian or regionally as Tamil came up in many interviews and was debated between participants (See Chapter 4). Although most interviews described tensions using the terminology of North and South, the idea that North Indian is Aryan and South Indian is Dravidian surfaced in some interviews. Even in interviews where this terminology was not directly used, participants alluded to the Aryan/Dravidian discussion, suggesting that it initially defined the North/South divide. Many scholars have also suggested that the history of Aryan/Dravidian has informed current debates regarding North/South divide in India (Bhatia, 2007; Jacob, 2009). In other words, the internal politics of these debates are similar - they place North and South India as opposing and separate. Regardless of terminology, most conversations reinforced that North and South India had different histories and often, these histories were forced together because of colonial occupation. Lakshmi and Arvind argued about the importance of identifying as Indian or Tamil, and which should come first. This was situated in the larger argument of North vs South Indian. Arvind said these differences were important, while Lakshmi said they needed to overlook them for unity. Both participants, in arguments referenced the 
complex history of Tamil Nadu in relation to India as well as the impacts on the diaspora abroad. (See Chapter 5).

While broadly, participants and experts discussed the Indian community as "brown" or "in-between", they also pointed to variations within, especially on the issue of dark and light-skinned Indians. Both Koshy (1998) and Bhatia (2007) stress the tensions between dark-skinned and light-skinned Indians often embodied in as North vs. South through categories like Aryan and Dravidian. In fact, Bhatia (2007), building from Koshy (1998) found that Indian Americans justify their links to whiteness through identifying as "Aryan" and characterizing it as the true superior race. Koshy (1998) describes that the links to "Aryan" are intricately weaved into Hindu, Indian diasporic narratives.

Some participants identified as "Dravidian", meaning from the south of India or as South Indian. Divisions between "Aryan", broadly North India and "Dravidian", broadly South India have been significant to Indian politics (Koshy, 1998). Koshy (1998) also mentions that 'South Indian Tamils', more than other groups, have overwhelmingly used the concept of Dravidian identity. Aryans are often viewed as more "white," while Dravidians are viewed as more "black" (Koshy, 1998). Those who identify as Dravidian describe those in Tamil Brahmin communities as Aryans who invaded from the North and were often lighter skinned and usurped local positions of power to subjugate Dravidians (Koshy, 1998). In fact, a few participants that I interviewed hinted at this association. While most discussed the issue as a North/South divide, a few participants mentioned that Tamil Brahmins were Aryan, not Dravidian. Some participants even went as far as to say that people from the South, Dravidians, were often more educated than those in the North. 
But while the terminology of "Aryan/Dravidian" only surfaced in some conversations, many discussed the issue as North vs South or used these terms interchangeably. Many participants brought attention to the racism that they felt for being dark-skinned, having non-white features or exhibiting non-white aural markers through language or accent. In other words, certain accents made them aware that they were not white, but also made them aware that they were not North Indians. Similarly, speaking Tamil in broader Indian communities, for some, was a marker of difference, and sometimes created instances of active discrimination through threats. Some also mentioned that being Tamil was marked by language descriptions like rough and uncultured. Yet, it was also described as skin color and linked to whiteness. Santhya described her frustrations with how Indian communities deal with color, first referring to being dark, which she connects to being Tamil:

Even today I was reading some essay. A woman was writing about her experience being made fun of for being dark-skinned. I had to stop reading. People are in complete denial of who the Indian woman is what her skin looks like. Referring to it as "getting a tan" in the Western sense, we are just not addressing the audience. Getting a tan and being brown. Indians don't get tan.

She described this as linked to the domination of Hindi speakers in diasporic communities, where those who speak Tamil are often looked down upon. Language and skin color intricately connect for Santhya and many others within Indian communities (see Chapter 6). She said that North Indians, often Hindi speakers, seem to dictate the dominant 
narratives about Indians, with language and skin color. Sakthi and Vimala also connected markers of difference. They mentioned how their curly hair did not fit the narrative of straight, silky "Indian" hair, which they often saw with their North Indian friends (see Chapter 6). Sakhti then mentioned how they felt like minorities in Indian grocery stores, shops, etc. because everyone else was speaking Hindi around them. They would speak to one another in Tamil so that no one else would understand them and to purposefully mark themselves as non-Hindi speakers.

Some participants described that Tamils were marginalized in discussions of India as India was often defined by North Indian narratives through movies, popular culture, and even educational media. Tamil scholars criticize how other scholars have dealt with the Indian diaspora and Indian nationalism (Devadas, Vijay and Velayutham, 2008; Velayutham, 2008a). They argue that the context is often ignored regarding Tamil Nadu's history, and Indian communities are often discussed through North Indian lenses. Many interviews reflected this sentiment.

For example, beyond Arvind's discussion in Chapter 5, many participants mentioned how Tamil Nadu has a complex history and some semblance of identity before India existed as a nation-state. As mentioned in Chapter 5, pre-colonial Sangam Age literature gives cultural foundation for Tamil schools and Sangams in the US, thus highlighting a regional scale of Tamil first, and a national scale of Indian second, for cultural activities. But while Tamil Sangams and communities highlight Tamil events and programs, they still simultaneously connect to the national scale of India. While "Tamils" existed long before the colonial period (Selby \& Peterson, 2008), during struggles for independence, Tamil Nadu as a state through Tamil media outlets, focused on promoting broader unified 
national Indian identities to counteract and thwart British rule and domination (Devadas, 2008). The push for a regional split from the rest of India began only after independence. Many assumed that things would return to pre-independence and instead, felt that they were being subjected to an additional form of cultural imperialism, this time from the North (Devadas, 2008; Jacob, 2009)

Post-independence the Dravida Munnetra Kazhagam (DMK), which essentially stands for Dravidian Progress Federation, formed (Chidambaram 1987). The DMK promoted idealized Dravidian history and that Dravidians should have a separate state from Aryans and North India (Chidambaram, 1987; Jacob, 2009). Annadurai, then leader of the DMK, renamed "Madras State" to "Tamil Nadu" in 1969, promoting Tamil history and Sangam period literature to fight for separatism from what was viewed as Hindi-speaking domination (Chidambaram 1987). Language became important signifier for Tamil Nadu and boosted the regional opposition to Hindi-domination from the North (Chidambaram 1987). Protests against Hindi continued in the 1960s, becoming violent (Pinto 1999). These historic Tamil politics were reflected within the diaspora. For example, these are the protests that Lakshmi mentions in relation to her father. He, however, as she describes, he was a victim because he did not speak out against Hindi and was harassed and punished for it. She said his stance was important to unify India at the national level. His experiences have carried over to her own experiences living in the US and influence how she views her identities.

Since independence, Tamil Nadu has wavered between supporting Tamil Nadu politics and uniting with India to redefine India through a regional Tamil scale (Devadas, 2008). Tamil Nadu often wanted to define Indian, rather than be subsumed by Hindi or 
what was described as North Indian domination (Jacob, 2009). Devadas and Velayutham (2008) argue that the cultural dominance of the North portrays the South as backward or inferior (Devadas and Velayutham, 2008). While this is important for politics in India, it is also relevant to US Indian diaspora politics. Many participants said that they felt marginalized in the broader Indian community precisely because Bollywood, Hindi, North Indian food, or other items that were not significant to their experiences, represented Indians.

Many participants mentioned that they would reinforce their identities of being Indian or Tamil through watching films or listening to music. Many also mentioned that while Bollywood and Hindi music were popular in the US and seemed to represent the image of Indians abroad, they personally watched Tamil films and listened to Tamil music. Others, however, equated Indian with Bollywood music because that was the dominant narrative, even if it was not relevant to their own experience.

For instance, Anusha in Chapter 4 referred to the problem of being Indian rather than Tamil, “...my resistance to it is how people in America can lump me into a category or associate all these things that have nothing to do with me." Others agreed with this sentiment. Fathima said, "I think like people would think of Bollywood music or something. I know Tamil Bharatanyam, but that wasn't part of our lives... It was not as core to my identity." Even still, a few participants did associate Bollywood with their idea of being Indian. Nazeem, for example, sees Bollywood as part of her more hybrid, blurred South Asian identity, that she describes as desi. This blurs the lines between Pakistan, Indian, Tamil, and all of her South Asian/desi identities. Bollywood was a part of her experience growing up as desi. 
When I was a kid, I watched a lot of Bollywood movies. The classic 90s jams were a classic part of my childhood. Knowing what was going on in Bollywood was part of being desi to me - doing dances to songs at weddings was a big part of it.

But, others defined Indian by Kollywood films and songs. Film analysis has been significant in much research on the Indian diaspora (Alessandrini, 2001; Desai, 2004) and as Velayutham (2008b) argues, many scholars use film to understand the diaspora. Yet, it is often "almost always from the vantage point of Hindi films" (Velyautham, 2008b, 5). Many of the NEOTS functions highlight Kollywood and popular Tamil music, ranging from older songs to the latest hits, in programs through performances, skits, and shows. For example, in the 2016 and 2017 NEOTS Deepavali programs, pop stars from Tamil Nadu came to the US to perform popular songs for the event. Though many of these functions are represented as nationally Indian in the sense that they programs are described as Indian depending on audience, throughout programs, Bollywood is not significant. Once or twice in the 15 programs I attended, I heard a Bollywood song played, but the lyrics of those songs were purposefully changed to Tamil. As Devadas and Velayutham (2008) argue, the pervasive cultural dominance of Bollywood that also informs the Indian diaspora abroad marginalizes narratives and histories of the South. Thus, in events organized around regional identity such as Tamil, Bollywood is not relevant.

Some participants said they felt marginalized because they were Tamil. In general, this stemmed from experiences in India, but also carried over to the United States. Some participants like Arvind, described that racism in India is quite significant. In fact, he 
compared it to the white supremacy in the United States and the West. "In India, we (Tamils) are very discriminated against. They discriminate against color. If anyone is dark, they are looked down upon. That is, the North Indians look down on South Indians. That white supremacy you have here exists there." He said that this is in part why he does not go out of his way to be part of broader Indian communities. Sam also described instances where he felt actively discriminated against for being Tamil. He recalls one specific experience that happened in India:

I was in Mumbai for my Air India staff trading in 1966 and don't speak Hindi. The Shiv Sena ${ }^{37}$ group was getting prominence then. I was a paying guest in a home and somehow news spread and one evening when I got home, my belongings were out and the landlord said he cannot keep me in his house since the Shiv Sena group he'd informed him that they will burn his house if they accommodated a South Indian Tamil.

Discrimination to the level of Sam's experience was not commonly expressed, but did happen for some participants, though not as severely in the United States. Yet, it still seemed to carry over and influence how some participants interacted with other Indians in the US. Even Lakshmi, who identified as Indian primarily, said that she noticed times that she did not fit into certain Indian groups because she was not North Indian. But for

37 Shiv Sena is a far-right political party that promoted Hindu nationalism in the late 1960s and 1970s and discriminated against South Indians (Jayaram, 2010). Sam was not just a South Indian Tamil, but he was also a Christian South Indian Tamil. He described a few experiences where he felt persecuted as a Christian in Hindu-dominated areas as well. 
Lakshmi and others, this also connected to language. Lakshmi said that she feels bad because she cannot speak Hindi. "Coming from India, see our national language is Hindi, I feel bad that I never learned, because it brings unity, but I am 100 percent Tamil." In this moment, she identifies regionally as Tamil when discussing a broader scale of identity like Indian. This was especially connected to language. Earlier, she identified as Indian first. She emphasized one scale over another in a very hierarchical way. In some instances, she identified nationally, and while in others, regionally.

Diya, who also described herself with the larger scale of Indian because it was important for unity, also described herself with a smaller scale of Tamil when referencing language. At times, she had described herself as more hybrid, as an Indian-Tamil with blurred identities. But, in these moments, her identities were hierarchically scaled. She was Indian but became Tamil regarding language. Sound influenced how she scaled down. She said that she at times did not fit into broader Indian communities because she could not speak Hindi:

Here when you meet a fellow Indian, they automatically assume that I speak Hindi. I have to explain to them. Sometimes that is a little difficult. When you don't speak in the same language, it is hard to get close. They don't discriminate, but I feel like I should speak Hindi. There are a lot of North Indians....They assume that you know Hindi because that is expected. If you know Hindi it is a little easier to mingle. I didn't have the opportunity to talk fluently. 
Diya does not describe her experience as discrimination, but instead, places the blame on herself for not having the opportunity to learn Hindi. She, like Lakshmi feels like she should have learned Hindi. Others, however, are not as sympathetic. Many participants said that they were annoyed with the automatic assumption that they speak Hindi. Some said that it important to recognize that there are many other languages in India, not just Hindi. Jaya said that because of the divisions within the Indian community, especially related to language, she identifies regionally as Tamil more than Indian. She said that when growing up, other kids her age were often listening to Hindi and Bollywood songs, movies, languages in their homes, but she could not relate. "Hindi... that isn't my language."

In Chapter 5, participants like Anusha and Sakhti described how their experiences growing up were very different from their North Indian friends, which is why they identified regionally as Tamil instead of more broadly as Indian and much of this had to do with language. Madhavan describes that most people in the Indian community make similar assumptions about him as well. "Most of the people I ran into they are like hey do you speak Hindi or do you speak Telugu? First and foremost, yourself, you're from North India, and yea, I do think I'm going to be a Tamilian." He explains that in those situations, he identifies much more as Tamil than Indian. He said that in North India, people segregate South Indians and often distance themselves from them. Since he came to the US in 2015, he says that although he does identify as Tamil, he also sees that Indians have to be somewhat united in solidarity when they are a minority in a different country. Thus, these identities become much more blurred and hybrid. He is still Tamil, but also must be Indian or something in-between. In the US context, depending on the situation, he shifts between identifying as Tamil, Indian or both. 
But while some participants described these as only minor inconveniences, others described incidents where they felt active discrimination. Even Madhavan said that he experienced some discrimination for being Tamil. Many of these had taken place before they came to the US, but nonetheless, the experiences shaped the ways in which they interacted in diasporic communities as well. Santhya says that Hindi and expectations of speaking Hindi define the Indian diaspora, which makes her feel marginalized. "Hindi dominates the expat community... They are Hindi dominants. We already felt like a minority." She refers to the fact that she already felt like a minority within Western countries being non-white, described in Chapter 6. Yet, even within Indian diaspora communities, Tamils are still a minority and this is made apparent through various interactions that she has had especially regarding language and not speaking Hindi.

Some described this type of discrimination carried over from when they were in India and influenced how they saw their identities. Muthu, for example describes that he has experienced discrimination for being Tamil. However, he says that he was made more aware of this type of discrimination after growing up in Delhi as a Tamil.

Growing up in Delhi, definitely, there is a little bit of a North/South divide in India and that shows up in civil conversations and in bullying. Derogatory terms used against South Indians. And then you retaliate...But I've definitely been referred to as 'Madrasi' which is derogatory. When you hear a brother and sister talking in Tamil, the common insulting phrase that Hindi kids use "andla pondla ${ }^{38 "}$ and then it leads to arguments.

38 This is a phrase used to imitate the sound of Tamil in a derogatory way, but has no translation. 
Muthu was not the only one to experience this type of discrimination. Even Madhavan described these interactions. "It happened a lot in North India whenever I talked to someone, 'Are you Madrasi?' They classify. Other people do this. They classify most of the South Indians as Madrasis." While Muthu and others described the term "Madrasi" as derogatory, some participants directly identified as Madrasi, using it with a sense of pride. These participants said that they were proud to be Madrasi. Much like Nazeem described in Chapter 5 when she identified herself as Madrasi.

Those who did not use the term suggested that it was derogatory because it grouped all South Indians together without nuance. It suggested to them that all South Indians were the same because they were backward like "those Madrasi people, who spoke uncultured Tamil, and were all dark-skinned." The term "Madrasi" originated from the British Raj and was used during colonization to purposefully designate people from South India in a derogatory way (Arnold, 2010). It was appropriated by the Shiv Sena group (that Sam referred to earlier) in the 1960s and 70s to refer to and marginalize people from the South (Jayaram, 2010). Its colonial origins continue today in a form of mimicry. In this sense, it is a literal adoption of a colonial term used by those who were formerly colonized. As Bhabha (1994, p. 87) says, "mimicry repeats". “...colonial mimicry is the desire for reformed, recognizable Other, as a subject of difference that is the same, but not quite..." (Bhabha, 1994, p. 86). As Bhabha (1994) notes, it is the imitation of colonial behavior that keeps the legacy of colonialism alive. Thus, the same terms, words, and practices are imitated by former colonies and used in practice to repeat the othering and subjugating elements of colonialism. These terms have carried over beyond former colonies into diaspora 
populations. Participants said that even in the US, many in the diaspora still use the term "Madrasi." Sometimes, it purposefully used as a derogatory term, and other times, it is used as a term to describe South Indians, without awareness of its derogatory origins.

For participants, despite the colonial legacy, most encounters with the term were with North Indians. There was a consensus that if North Indians used the term it is much more derogatory than if South Indian use it. While most participants described discriminatory instances that happened in India, some described two things about the US. First, that discrimination in the US was still present, just often unspoken and second, that their experiences in India shaped the way that they saw broader Indian communities in the US. Some participants said that they have heard people call them Madrasi even in the US. 'Madrasi' also connected to language. Santhya describes this when discussing the internal politics of Indian communities.

Indians kind of micro-define themselves through caste, religion, etc...that tendency when people say everyone in the South is a Madrasi, that makes me mad and I need to rectify that. When Hindi speakers say 'oh when you go to the South they don't speak Hindi' and I'm like why should they? That is not their language.

Views on discrimination varied among participants. Some saw a North and South divide as extremely important. Many said this had to do with that North Indians looking down on South Indians, especially Tamils. When referring to North Indians, most participants referred to those who spoke Hindi from primarily Hindi-speaking states in North Central India, Delhi, or even Mumbai (which is in the state of Maharashtra where 
many people also speak Marathi). There was a consensus that those in Northeast India, places like Darjeeling, were different from those from North central India. But while some participants made these distinctions, others just referred to North India as a whole. Some said they had never interacted with North Indians before coming to the US or really known much about North India. Bharathi, for example, says she never spoke with North Indians until she came to the US. In fact, she said, she may have never even seen a North Indian before coming to the US.

Many participants said that North Indians described South Indian languages as hard or harsh-sounding. Also, they said that they felt that some North Indians saw Tamils as stubborn and inferior because they did not know Hindi. This sentiment was part of the unspoken discrimination, or microagression that they felt in the US. This intersects with the debate between Lakshmi and Arvind in Chapter 5. Some participants indeed, agreed with these sentiments about Tamils wanting to separate from other parts of India. Yet, others said that not learning Hindi and not trying to connect with the broader Indian community was a fault of those who identified as regionally Tamil first. Some described that these differences needed to be embraced to have agency within the Indian community and redefine Indian as Tamil. Others, said that there was no need to identify with the Indian community, and staying within an Indian Tamil community was most fulfilling and appropriate. Finally, some participants said that they did not want to be involved in any community so that they could avoid all drama and politics associated with Indian-American communities. These participants referenced these debates between North and South and said that they wanted no part of it, so they did not take part in broader Indian or even Tamil events or functions. 
Often, the Indian community was framed as a North/South divide by participants. Even beyond interviews, in my participant observation, the Tamil events I attended made distinctions between traditions in North India and traditions in South India. In Tamil festivals like Pongal, sometimes events reiterated that this was a Tamil festival, but still placed in the context of India. At events like the Cleveland Thyagaraja Aradhana, however, music traditions were highlighted within programs through maps and elaborated on during discussions (See Chapter 5). The divides between North and South India were reinforced through printed programs, events, and festivals.

\section{Othering within "Tamil" identity}

While participants discussed the effects of discrimination in the Indian community, often framed around a North/South divide, many also described frustration with Tamil communities for reproducing hegemonic representations of Tamils. They described this through intersections of religion, caste, scale, region, gender, sexuality, primarily/among many others. However, while these are important, for this dissertation, I focus on those that relate to scale and hybridity because these themes were most prominent in my data and analysis.

For example, those who identified at times with small-scale identities like Madurai Tamil, Coimbatore Tamil, and others, did so when they were speaking about marginalization within the smaller scale context of Tamil Nadu. Some even went as far as to describe their village as important to how they saw Tamil, because it was with that village that they felt most comfortable identifying (See discussion in Chapter 5). Both Raj and Maari in Chapter 4, used scale to strongly identify with their village or town. When 
describing the importance of Tamil identity or instances of discrimination within broader Indian communities, participants reverted to a larger-scale description of Tamil identity, referring to just Tamil. Yet, when discussing marginalization within Tamil communities, they would then add nuance again with villages, regions, cities, or other scales of identification. They often connected caste and religion to these descriptions, which I discuss further in this section. Finally, many participants stressed the importance of accent and language when navigating various identities. In Chapter 4, 5, and 6, I showed how many described accent as important to a variety of identities and experiences with discrimination. In fact, accent came up again in relation to how participants viewed discrimination and othering within Tamil communities. Language was more important to discussions of discrimination within broader Indian communities, but accent became more important within Tamil communities. In Chapter 5, I already discussed how participants associated regions like Madurai or Chennai with specific qualities based on accent. Many said that they worried about how their Tamil sounded after so many years living in the US or growing up in the US. More importantly, those who struggled to speak Tamil said that they felt marginalized for the way that they spoke Tamil. Jaya, for example, said that her cousins often made fun of her for her American accent when speaking Tamil. She said this caused a lot of emotional turmoil as they constantly ridiculed her for not speaking Tamil properly. Sound, more specifically accent, was important to her experiences of being othered and was in many ways hybrid. She said was made to feel as if it was her fault and that she was bringing shame to her family for the way she spoke. She could not be fully Tamil. In this way, she felt in-between identities. Hybridity is not just indicative of blurred identities, but also suggest that identities are in-between binary categories. 
Many participants expressed similar sentiments. While some said that they could not always identify as Indian because they did not speak Hindi, others said they could not always identify as Tamil because they did not speak Tamil or did not speak it well. They were often in-between. Finally, they could not always identify as American because they looked different or sounded different than white Americans. Almost all participants described one or more identities in which they felt they were not able to fit fully, creating in-between identities. The rigid categories established by colonialism and discourses like Bollywood, which many participants used to conceptualize their identities when they identified using traditional, hierarchical scales, made it difficult at times, to navigate identities. Hybridity is useful as an academic concept to investigate such experiences, but, in this study, was often theorized by the researcher rather than the participants. Participants often initially described identities as scalar, hierarchical, and separate, using very traditional scalar terminology. But, when describing life experiences, I noticed that they shifted to more multiple and hybrid descriptions of identity. Hybridity was important precisely because it captured the in-betweenness of identities that did not fit colonial binaries. For example, participants like Nazeem or Vimala in Chapter 4, described difficulties navigating these identities because they often felt in-between them. Sometimes, they described them as multiple and used multi-scalar descriptions of identity like South Asian, Indian, American, and Tamil simultaneously. At other times, they described themselves as a blurring of these identities or alternatively, in-between them. For many, they felt in-between because they had to choose or continuously reproduce characteristics, like speaking a certain way, that allowed them to fit into certain identities. 
This was very much tied to discrimination and othering. For example, many second generation participants said that they felt some discrimination through being othered for their accents. Jaya says that even though family made fun of her for how she spoke Tamil, when her cousins spoke English with Tamil accent, she never made fun of them. This suggested that she could not really be Tamil, at least not in the way that her family wanted her to be Tamil. Others had similar experiences. Durga, for example recalls these experiences often. "I go to English for specifics. English is much better than my Tamil. When I speak Tamil, people think it's cute. Look, she is trying even though she is American." No one takes her seriously. She says she doesn't feel American or Indian because of this and instead feels as if these identities are blurred. Sakhti described this happening to her as well. She felt othered because she was constantly hyper-aware of her accent. She says that others constantly criticized her for how she spoke Tamil.

I do speak Tamil, but my aunts, uncles, and cousins ridicule my accent. I'm like look people let's hear you speak English. Even for my parents, their Tamil has devolved into liberal English. They morph into a Tamil English thing. That is the Tamil that I'm used to. My parents watered it down. I can understand my family for the most part. I can't understand formal Tamil.

She said her mom signed her up, along with her sister, with a tutor to teach them formal Tamil when they visited India after moving to the US. But, she said, they did not keep up with it and only went briefly. For many who grew up in the United States, accent, when speaking Tamil, was a significant source of concern. It was a marker of difference that kept 
them in-between identities. For others who did not speak Tamil, language was also a significant source of concern. Some participants said that they felt isolated or othered from being Tamil because of these factors. Most second-generation participants said that they wished they spoke better Tamil or had less of an American accent.

Many described that growing up pre-2000, speaking Tamil was difficult because the community was not as large as it is now and did not have the same resources. In other words, they were being ridiculed or othered for something that they could not control. Many described that it is easier to fit into the community now with more resources. For example, Sakhti had to go to India to learn Tamil, but now, Tamil schools are appearing throughout the US. Some described a divide in the community between those growing up before 2010. Those who cannot speak well now or did not have Tamil resources to participate in are deemed more American or less Tamil. This made many participants feel left out or othered. Anusha says that she has recently noticed the difference after she started attending a Tamil Sangam.

Recently, I started attending Tamil Sangam events. Right now, there is such a huge critical mass of Tamil people. I feel like a lot of Indians are a lot wealthier, who have grandparents living at home. They picked up Tamil a lot easier...They don't realize there was a generation that came before the years 2000....It doesn't integrate our experience within their organizations as well.

When she describes "our" experience, she refers to what many participants described - a disconnect between what Tamil generations experience today versus what 
participants experienced in the US prior to 2000 or even 2010. This disconnect was related to how many participants felt othered within the broader Tamil community. Many secondgeneration participants said that they did not have access to many Tamil people, Sangams, or schools when they were growing up. They grew up in a very white or mixed environment with people from various parts of India. Simply put, they experienced discrimination in these mainly white spaces for being brown and now felt that they are othered within these communities for not being Tamil or brown enough.

Beyond accent, participants also described how caste and religion reinforced othering and discrimination within Tamil communities. Radhakrishnan (2003) shows how in the US, upper-class Hindu narratives define Indian communities. These communities often silence voices of Muslims, Christians, Sikhs, and others. He shows how diaspora communities can become very Brahmin-centered, silencing non-Brahmin Hindus as well. Throughout the interviews, participants connected Indian and Tamil identities and even scales to religion and caste (i.e., as mentioned in previous sections, the idea that Brahmins were from North India, a subnational scale, invading local and regional Tamil societies). Scholars like Radhakrishnan (2003) and Bhatia (2007) have described how these contribute to ideas of whiteness within Indian communities. In other words, caste and religion can reinforce mimicry in the US, which I describe later in this chapter.

When describing the "sounds" of Tamil identity in Chapter 4, many participants mentioned Karnatak music, or even specific accents associated with Brahmin Tamil. Again, many linked this to North India, suggesting that Brahmins came from the North and influenced local and regional politics. Some also expressed concern that communities represented Tamil as primarily urban, Brahmin, or upper class through language, accent, 
and even music. Jaya, who grew up in the US, says that she still recognizes that caste informs Tamil identities, especially through accent. "When it comes to Tamil there are different regional accents in Tamil. There are caste accents too." She described a few instances using where she heard people use specific words that indicated caste, but also said that these intersect. Regional accents can define specific areas where someone is from, but caste accents can show this as well. Some participants described the use of "jalum" instead of "thanni" for water. Thanni is a non-Brahmin word, while jalum is Brahmin. A non-Brahmin would not say jalum to describe water. Prisha also described how caste and region divide Tamils especially in the way that they speak. Tamil is not just Tamil but is divided along region and caste. She describes how tones, inflections, and even words are different.

They are Tamil based on caste. We are Tamil Brahmin... the words would be different...the tone and everything would be different. I visited the city my mom grew up in and we had a cab driver. I couldn't understand him at all. The way he annunciated everything. It is very strong in each city. Especially if you go deeper into like the Tirunelveli district, they have a very strong accent.

For Prisha, accent was not just based on caste, though that was significant, it was also based on region. In Chapter 4, I described how certain participants felt marginalized based on regional accents. Some had described that their Chennai accent marked them with negative qualities, especially with how well they spoke Tamil. Yet, others, who were from areas like Madurai, where Tamil was regarded as more sophisticated, described that they 
felt people also associated them with negative qualities. Constant tensions between scalar identities within Tamil Nadu were significant and religion and caste intertwined with these Tamil identities as well as broader Indian identities. Some participants mentioned that Brahmins would be more likely to live in wealthier areas of Tamil Nadu and they would similarly be more likely to live in wealthier areas of the US.

Participants like Prisha, who described caste as important to Tamil identity, attends festivals like the Cleveland Thyagaraja Aradhana and considers Karnatak music important to being Tamil. But, Pandian, Panthanakan, and others mentioned that music at festivals like the CTA are meant just for Brahmins. They said they might consider visiting this festival but would not go out of their way to attend it because it is meant for Brahmin Tamils. Others who did not attend these music festivals mentioned that they could not understand Sanskrit or that Sanskrit had North Indian origins.

Participants like Pandian or Panthanakan also said folk music was important to Tamil identity, but many participants, even second-generation, described that Karnatak music was part of Tamil identity. In fact, when describing sounds related to being Tamil, many participants described Tamil as related to Karnatak music. Yet, as described above, Karnatak music intertwines with upper caste, mostly Brahmin Tamil narratives. In Chapter 5, participants described varying views on the Karnatak connection to Tamil identity. In fact, many described it as Brahmin-centered, having little or nothing to do with lower castes or village life. Some participants well-versed in music, described that village or folk music was often erased by narratives of Brahmin, Karnatak music in many events and festivals in the United States. 
Karnatak music was also to region. Karnatak music is more dominant in certain areas of Tamil Nadu. It is prominent in urban centers like Chennai, but also in cities like Thiruvaiyaru, that host the Thyagaraja Aradhana to honor St. Thyagaraja (the CTA is the sister festival). Chennai's six-week long December music season, centers on Karnatak music and is the dominant classical musical celebration in the state (Viswanathan and Allen, 2004). Cleveland, OH's Cleveland Thyagaraja Aradhana, the second largest Indian classical music festival in the world, is connected to Chennai's music festival as performers from Chennai come to Cleveland and vice versa (Viswanathan and Allen, 2004). Simply put, some of the largest celebrations of Karnatak music in Chennai and Cleveland, $\mathrm{OH}$ are both urban areas. Some participants mentioned that these areas shape narratives of Karnatak music and Tamil communities in the US.

Many participants described the importance of Karnatak music to US Tamil communities. They mentioned that they loved to attend concerts or knew that it was a part of being Tamil. Yet, Karnatak music is also Hindu. Even some non-Hindu participants I interviewed connected Karnatak music to Tamil identity, suggesting that they often think of Karnatak music in relation to Tamil. However, they also suggested that it was not a part of their life experience or identities. Even Hindu participants described that upper caste narratives can define Tamil communities. They also said that it was not important to their experience of being Tamil. Raj, for example, when discussing the Cleveland Thyagaraja Aradhana, said that it was not significant to his experiences or to many other Tamils that he knew. "It is mostly a caste thing. You would see upper class people do that Thyagaraja organizing. Only certain types of Tamil people are participating in that. More like a Tamil 
program - but I don't see everybody going to that. It is one of the functions dominated by the upper class Tamil."

In Chapter 5, I discussed how some participants mentioned that many Tamils do not understand Karnatak music and that it is only for a certain group of people, Brahmins. They described frustration with the fact that the music was primarily associated with Brahmins and that was defining Tamil identity. In their views, this kept performances involving village or folk music silenced. But not all participants had issue with it. Some said that as an art form, regardless of where it started, what it represents, it should still be respected.

Some participants, when describing Tamil sent video, audio, or image references to folk music and particular instruments, including the parai, a drum that uses animal skin. It is important to many villages but described as offensive to upper castes. One of my expert interviews, who specializes in music at University of Madras, said that he recently brought drums made of animal skins on campus. Previously, they were banned, but he said that he is trying to change caste politics and the way that certain sounds are viewed. This drum, he said, is taboo, especially in places controlled by upper castes. So, for example, he said, you might never see this instrument in a performance like the Cleveland Thyagaraja Aradhana. Those in the CTA would consider this drum offensive.

Beyond issues with caste, many Muslim and Christian Tamil participants said they felt broader Indian and Tamil events often focused on Hindus - this in some ways, connects to whiteness, which I will describe in the next section. They described that they felt marginalization in broader Indian communities, but also within Tamil communities. In fact, many even said that they associated being Indian with being Hindu even if they were not 
Hindu. ${ }^{39}$ Participants rarely described Islam or Christianity as associated with being Indian. Some described it as associated with being Tamil, but not Indian. Others associated it with South Indian, but not in the national scale of Indian.

Fathima, Nazeem, and Suhail for example, made distinctions between the way that their Hindu friends experienced Tamil and the way that they experienced being Tamil as Muslims. Fathima and Suhail did not directly or openly associate being Tamil with being Hindu. Fathima especially mentioned that being Muslim influenced the way that she viewed being Tamil - it was blurred and hybrid. For Suhail, being Tamil was integrated into all his other identities, including Muslim - also in a very hybrid, blurred way. Nazeem, on the other hand, mentioned that the first time she met other Tamil Muslims, she was shocked. She said, “I didn't know y'all existed (referring to Tamil Muslims). That's cool. What's that like? That is news to me. That there were even Tamil Muslims at all. All the people we knew were Hindu." By default, Nazeem was only surrounded by Hindu Tamils and had never met any 'Tamil Muslims' until she was older. This influenced how she viewed both Indian and Tamil communities in general - as very Hindu.

Participants, both Hindu and non-Hindu, reflected Radhakrishnan's (2003) sentiments that both Indian and Tamil communities were defined by being Hindu. Some participants also echoed his sentiments that this was problematic because it marginalized other religions, especially Islam, which was currently associated with non-whiteness in the US. It connected to problem of whiteness or "brown privilege" in that many Indian and Tamil communities actively tried to distance themselves from additional markers of non-

39 In Tamil Nadu, Hinduism in the dominant religion with 87.58 percent of the population practicing Hinduism. Christianity makes up around 6.12 percent and Islam 5.86 percent (Census of India, 2011). 
whiteness like Islam, a religion marginalized in both the US and India. Yet, as SAALT (2017) indicates, overwhelmingly, Muslims and Sikhs experience the most hate crimes in the Indian diaspora. As Sakthi, who was raised Hindu, describes of Hindu communities:

Especially in this climate, I've noticed this in Indian people, there is a general weariness of Muslim people. The whole Trumps ban of Muslim people. It doesn't bother the older generation of Indians. They are like, it is not affecting us. That is not how this works people. Pretty sure this is how it starts with any major conflict.

Even Sakhti equates Indians with being Hindu and non-Muslim. She brings attention to the issue, but still refers to Indians as Hindus. Which, in many ways, does describe much of the US Indian community. Christian Tamils said that they felt that it was easier to integrate into the US because there were so many churches. Though, for some, these

churches were often white, like Ruth's church. Yet, associating with Christianity was a marker of whiteness as it was the dominant religion in the US. Most of the participants that I interviewed however, were Hindu. Yet, it did not define all the Indian community. Within both the Indian and Tamil community, many participants felt marginalized based on religious affiliations. For those who were Muslim, they felt marginalized both in Indian and Tamil communities, but also in the context of the broader US.

Problems of whiteness in the Indian diaspora

While discrimination and othering are weaved Indians and Tamil communities in the US, these communities also deal with the problem of whiteness or what Bhatia's (2007) 
"brown privilege." For this section, I build on earlier discussions to show how Indians more broadly, and Indian Tamils in the US are connected to whiteness. I show how whiteness contributes to discrimination within Indian communities. In instances where participants felt discriminated against in Indian communities, they described themselves as Tamils, relying on regional scales. Yet, when referring to themselves in relation to other communities, often considered themselves Indian, relying more on national scales.

Participants mentioned discrimination against dark-skinned Tamils. Being darkskinned is a marker of difference that include items like language or accent, and sometimes caste. As demonstrated in the previous section, accent also informed caste as different words and accents reinforced being Brahmin. Many participants also suggested that Brahmins are often lighter-skinned than other Tamils and make up the majority especially in events like classical Karnatak music festivals. Some participants also mentioned that Brahmins were Aryans that invaded the Dravidians, linking them to whiteness.

Scholars like Bhatia (2007) and Koshy (1998) frame racism within Indian communities through lenses like ethnicity and assimilation. Yet, it is also a product of mimicry in a post-colonial world. I recognize that the context of the United States is not directly post-colonial, but rather a product of settler colonialism (Inwood \& Bonds, 2016; Veracini, 2013). However, as Veracini $(2013$, p. 3) suggests, settler colonialism reproduces the conditions of colonialism, but instead of reinforcing difference, it erases it. In other words, it constructs the colonial as if it has ended with the settler colony. Yet, the effects of colonialism continue to shape current policies, practices, and borders around the world. The framework of settler colonialism is useful to understand social conditions and issues of 
race in the United States, yet, the links to colonialism are still meaningful and important because the Indian diaspora has been shaped by them.

As I described in Chapter 6, mimicry is important to theorizing the "problem of whiteness" in the Indian diaspora as it demonstrates how the language and binaries of colonialism are still reinforced within diasporic/migrant populations. For example, as Jazeel (2006) suggests, members of the UK Sri Lankan diaspora can be products of colonization, but also take on the role of the colonizer. Jazeel (2006) discusses mimicry and its connections to hybridity in the Sri Lankan diaspora. As mentioned in Chapter 2, the official Sri Lankan Association supports and reinforces gender roles set by colonialism before independence. The women in the Sri Lankan diaspora community see themselves as guardians and protectors of underprivileged. They view themselves as exoticized and Orientalized, but also play the role of the colonizer by exoticizing Sri Lanka. In other words, their binary views of Sri Lanka reproduce 'Western' values that separate them from those "others" in Sri Lanka. They have taken on the role of the colonizer, viewing themselves as above the oppressed.

Similarly, many participants mentioned that Indian communities see themselves as better than or more important than other minority communities. Some participants even described how Indian communities in the US reproduce colonial binaries, divisions, and markers of difference. The United States is not connected to colonialism in a direct way but is very much associated with whiteness and reproduction of colonial norms (Bonds and Inwood, 2016). In fact, instances of racism directed at other Indians within Indian communities mimic colonial categories and binaries (See earlier discussion of "Madrasi"). Outside of the Indian community, mimicry reproduces a direct racism and disengagement 
from other minority communities in the US through reproduction of what Chand and Tung (2014) describe as "white, Christian values," not limited to Christian communities. As Radhakrishnan (2003) and SAALT (2015) suggest, Hindu communities are also guilty of mimicking "white Christian values" and displacing Muslims and Sikhs.

For Chand and Tung (2014) Hindu and Christian values align much more when "other" religions fall into political spotlight. In this respect, the concept of 'misrecognition' can also be problematic. For example, in Hopkins et al. (2017) study, the danger of using misrecognition lies in that it subtly implies those who are not Muslim do not deserve discrimination precisely because they are not Muslim. In other words, being Hindu or Sikh is better than being Muslim. While the concept of misrecognition is incredibly useful to understand broader implications of Islamophobia, it should also be handled with caution. Hopkins et al. (2017) use misrecognition as a tool to understand othering, but many nonMuslim Indian communities in the US actively separate themselves from being Muslim, black, or as any other minority (Bhatia, 2007; Koshy, 1998).

During interviews, participants, often second generation, described "weariness of Muslims or minorities" and also mentioned what they referred to as "racism" in US Indian communities. They described it as Indians in Indian communities "thinking that they are white." Sakthi, for example, described this as an issue that divides communities.

Most Indian people identify more with white people than people of color. I feel like there should be more solidarity between people of color. That is a general theme. But I kinda feel like it is definitely something that is more prominent in people that are here and they are usually in the upper middle class. 
Sakthi mentioned that this connects to caste and class as those who come the US are often upper caste and upper class as they had more opportunities in India. Sakthi says these are the people that take the spotlight and represent Indians in the US. They are also more likely to speak English well. Speaking English with an Indian accent is a marker of difference that is exploited and emphasized in US popular media (Dave, 2013). Participants like Sakthi referred to not just divisions in the Indian diaspora, but also in the Indian Tamil diaspora. These divisions are underscored through accent and music - like certain accents or music linked to Brahmin Tamil, reifying narratives of upper class, caste, and closer connections to whiteness (Radhakrishnan, 2003; Bhatia, 2007). Whiteness was associated with certain characteristics like high-paying jobs, education, or command of the English language. Some participants mentioned that Brahmins and upper-class Indians and Tamils in the US were more likely to exhibit these characteristics. Bonds and Inwood (2016, p. 719) refer to the "social condition of whiteness", or conditions like wealth, education, etc. that are typically associated with privilege. ${ }^{40}$ Though racial constructions in the US have changed over time, many scholars suggest that the Indian diaspora was labeled the "model minority" because of economic status, giving the impression that they are "whiter" or more "American" than other minority groups (Bhatia, 2007; Safran, Sahoo, and Lal, 2008).

Bhatia (2007) describes that in his studies of Indian diasporic communities, there was an overwhelming tendency for participants to identify more with whiteness and

\footnotetext{
40 Bonds and Inwood (2016) do argue that scholars should be examining what lead to these conditions, rather than examining these social conditions. However, my analysis is more focused on recognizing that this condition exists within the Indian diaspora, but also acknowledging that it is complex and nuanced. Therefore, it is not in the scope of this dissertation to analyze the structures or institutions that lead to such conditions.
} 
Americanness (he mentions that participants used this interchangeably at times) than with anything associated with communities of color. In fact, Bhatia (2007) mentions that there was a concentrated effort to not identify with or as communities of color. Whiteness is often linked to "success" and characteristics that are associated with dominant, normative white groups (Bhatia, 2007; Dyer, 1997). So, it was not a surprise when I found for many, that the connection to whiteness was not just associated with skin color and did not always garner responses on discrimination, but instead elicited responses related to sound or aural markers.

Not all participants referenced the issue of whiteness within the Indian diaspora, however. Less than half of participants did, and the majority of those were secondgeneration. But, as Sridaran from SAALT suggests, issues of whiteness within the Indian diaspora are becoming more relevant as hate crimes for South Asians continue to rise. Sridaran (2017) said that connecting communities of color is a goal of SAALT:

That is one of the reasons we wanted to be really explicit about racial justice in general. We wanted to align with other communities of color. Help people see the similarities and our oppression is in relation to others that have faced a lot more violence. The surveillance program focused on Muslims now, that was designed to break down the Black Liberation Movement.

She discusses that minority communities are connected to one another through marginalization and making South Asian communities aware of this, might be helpful to combating racism on all fronts. Sridaran referred to incidents that made headlines in many 
US Indian community news outlets, specifically, with what happened to Suresh Patel.

Suresh Patel was an elderly Indian man beaten by police because he was misrecognized as black. She mentioned that suddenly, because this incident had direct impacts in the Indian community, Indian communities, briefly became more concerned about what happens to other minority communities. As one participant I spoke with described of Indian communities, "now that it is affecting me (referring to Indian communities), suddenly I care about this issue, but only how it affects me."

Arjun Gupta, a second-generation actor, and Akash Singh, a second-generation comedian in the US, also highlight the Suresh Patel incident in their 2015 podcast American Desis, that discusses issues in Indian-American communities in the US. But Gupta and Singh (2015) take this conversation further to point out the gap in how different generations of the Indian diaspora view whiteness, suggesting that is a larger problem for the immigrant generation.

AG: One thing I want to address is that there is a strong undercurrent of racism in the generation above us that are South Asians here... We strive to whitetify ourselves to the point that we distance ourselves from other minority issues as if that's not ours..."

AS: You know what is funny about our parents' generation, a lot of people, not speaking to our parents specifically, but we see a lot of racism toward dark-skinned people. Not just darkskinned Indians, although that definitely exists... White people do not see us as not being minorities. They may have a good perception of us right now because we are doing pretty well financially and professionally, etc etc., but especially 20 years ago when we didn't have all 
that and the only example of us was Apu, they didn't see us differently than black people really...I mean if I'm a racist white dude and I'm like I hate all these colored people except Indians?... We are all in the same boat...

Sridaran, who also was interviewed as an expert on the show, acknowledged in our interview that there is a generational disconnect between members of the Indian diaspora on the topic of other minority communities. She mentioned that recently, SAALT hosted a learning event to educate and inform diaspora members of the importance of working together with other minority communities.

We know it needs to be an intergenerational conversation, so we focused our young leaders on it...we felt if we are shifting our mission toward a commitment to racial justice that is one of the first things we have to do is address that issue (whiteness and anti-blackness) within our community.

Essentially, SAALT brought in professors, scholars, and organizations to create learning opportunities for the South Asian community. This included time for conversation where participants and leaders shared experiences and stories that Sridaran described as quite personal, intense, and difficult. "I think almost every single one of us cried at some point during that institute, talking about our own experiences talking about anti-blackness in our own families."

Anti-blackness is a significant issue in South Asian communities but has only recently being addressed (Bhatia, 2007; Sridaran, 2017). Both SAALT and some 
participants acknowledged that this is a significant issue within Indian communities. While misrecognition and racism from white Americans are concerns for many, they often linked this with racism within the Indian communities. Santhya, Sakthi, and others connected these experiences of marginalization within Indian communities to how Indian communities treated other people of color. Many participants who experienced and recognized discrimination were more likely to identify with the struggles of other minority communities.

Santhya, for example, said that the prejudice against dark-skin bridges both Indian and black communities. "My whole childhood, I experienced racial discrimination. At this point, I feel that I have a right to be anywhere in the world. If I see anyone being victimized, I would stand up for them." She notes that Indians in the US experience far less discrimination than other minority communities. She describes that in the US, "The bulk of the racism is directed against black Americans. At the end of the day, everyone of color is the same. They aren't any different." She clarifies to say that this is in terms of their humanity, but also acknowledges that Indians often ignore other people's experiences and have better treatment. “Many Indians feel that, 'Oh because we are treated better than blacks we should distance ourselves from them.'”

Most participants who discussed "Indian racism" described that racism within Indian communities was both related to dark skin in Indians, but also directed at other communities of color, mainly black communities. Sakhti said that because her husband is black, she has struggled continuously with her family. She says that their racism is at times quite blatant, but also subtle. 
I've seen passive racism toward John by Indian friends and family. His dad is very fair-skinned, but his mom is darker, and curlier hair. And I remember at the wedding, so many of my parents' friends said John looks Indian and his dad looks Indian, they could sit on the Indian side. Even my mom said, John looks Indian, we don't have to tell our Indian friends that he is black... I'm like oh my god.

Sakhti said that her family has experienced racism as dark-skinned South Indians, yet still reproduces racism toward other minorities. Though most conversations on racism against black communities were with second-generation participants, first-generation participants also discussed it. Arvind, for example, after describing the racism he felt as a Tamil, described a specific instance that he said he could not forget. When working at a university, he said he had an incident that forced him to think about the Indian community in general and how Indians represent themselves. "A black girl who is very brilliant told me you guys won't talk to me because I'm black. You are more prejudice than white Americans." He said this comment really made him think about Indian communities in general. I did not say this to him, but it was interesting that he qualified her by mentioning that she was very brilliant. As if prejudice was already present and he was trying to figure out how to deal with and acknowledge it.

In most events and performances, these issues of "brown privilege" in relation to other non-Indian communities were not as clear. Issues of prejudice were present in festivals like the CTA. Yet, these were also intertwined with caste, religion, and language. As Raj said, "only certain kinds of Tamils" attend the CTA. These issues were not as clear-cut as black and white, nor could they be dichotomized in communities as external versus 
internal issues. They were very much hybrid and blurred issues. Issues of prejudice between dark-skinned Indians and other minority communities overlapped with one another. These issues were not the same, but they did connect and echoed broader connections to whiteness and "brown privilege" as Bhatia (2007) suggests.

In many ways, "brown privilege" and issues of whiteness within the diaspora were connected to mimicry. The diasporic subject, a product of colonization, never becomes white nor maintains the full privilege of being white; yet, mimics colonial power, gaining more than they had before, as Bhabha (1994) suggests. Many participants said that racism is reinforced with the mentality of "people have it worse than us so we should feel good about our position." In the US context, a marginalized group tries to mimic white Americans, yet, do not reap the benefits of being white. The Indian subject within the US is a "subject of difference that is the same, but not quite..." (Bhabha, 1994, p. 86). Drawing again from Bhabha (1994), it is the imitation of whiteness that then reinforces the same power structures. It is also important to recognize that not every Indian in the Indian community has the same experience and this depends on situation and context. For instance, Indians, both the "model minority" in some cases, and simultaneously now the fourth largest undocumented group of immigrants, will not have the same experience. In fact, these experiences will differ significantly. Nevertheless, issues of whiteness pervade in the overall representations, discussions, and everyday lives of both Indian and Tamil communities. 


\section{Summary}

Each participant described racism within broader Indian communities, but also in that Indian communities as a whole do not identify with other communities of color. Nevertheless, while Indian diaspora communities in the US do experience a significant amount of racism as shown in Chapter 6, some also reap the benefits of being part of a privileged group that often identifies more closely with whiteness than with color. While the primary focus of this chapter is discrimination within Indian communities, elements like sound, scale, and hybridity are still informative. In terms of scale, participants were much more likely to identify as South Indian or even Dravidian in relation to what they perceived as North Indian discrimination. Yet, they also identified with a smaller scale of Tamil when referring to instances of language or even skin color. In the broader Tamil community, participants were more likely to identify with even smaller, more local scales like with a particular village or city when describing the marginalization they felt in broader Tamil communities often connected to religion and caste.

These experiences with scale were also hybrid as the binaries established by colonialism also created instances where participants said they felt in-between. They did not always fit into specific, clear-cut scales like South Indian, Indian, South Asian, Tamil, or others. Accent was significant to this hybrid experience for many second-generation participants who felt othered by the way that they spoke Tamil. Some felt in between identities as they could not be fully American, yet also could not speak Tamil in a way that made them fully Tamil,

While internal discrimination within Indian communities is important, it is nevertheless connected to outside discrimination within the broader US. Whiteness and the 
privilege of "brownness" were incredibly pervasive in both experiences of discrimination in the US, but also within Indian communities. It also informs how Indian communities view other communities of color. In the final chapter, I discuss this further along with the implications of the last four chapters to demonstrate the politics of identity within Indian Tamil communities in the US. 


\section{Chapter 8: Conclusions}

January 11, 2017 (9 days before Trump's inauguration)

It was 11:30am and I had been sitting on a bench for hours outside of customs in Washington DC, where my dad had been held up. 'Maybe my dad's accent is too thick for customs? Does he sound American enough?' These paranoid questions had manifested over the last 5 weeks in India. Looking brown is one thing, but sounding brown is another. If you look 'brown' and sound American, sometimes security does not pull you aside or harass you. But looking brown and sounding brown can be a beacon that draws unwanted attention. He had spent the last few weeks speaking in Tamil, so I worried that his accent was thicker. These thoughts consumed me in great part because I had been studying the relationships between sound, identity, and discrimination. Simultaneously living through the very experiences that I was investigating, brought a heightened awareness to the importance of sound.

When he finally emerged from customs he told me that he would have to look into US citizenship. He didn't have to say anything else. I knew what that meant to a person who said he "would be Indian until he died" - it was like he would need to give up a part of his Indian identity.

Less than three weeks later, Donald Trump called for an immigration ban from seven countries majority Muslim states. People from the seven countries and beyond were signing away their green card rights. Like many of my participants, I reacted to this 
incident with fear and dread. I immediately wondered - could that have been my dad? Shortly thereafter, a shooting in Kansas made headlines. A white American man saw two Indians in a bar and thought they were from Iran. He told them "get out of my country," and then pulled out a gun and shot them. Alok Madasani survived, but Srinivas Kuchibhotla died leaving behind his wife who subsequently had to battle deportation because her resident status was tied to her husband. The tragic irony of current US politics was clear to me - her husband was murdered for being a brown immigrant, and yet, instead of aiding her, the US government deemed her "illegal" and tried to deport her. And while my empathy was present, I still couldn't help but personalize it, thinking that it could have been my brother or my father who were shot. Later I would learn that many of my participants had reflected similarly on this incident. While these incidents do not all occur simultaneously, they nevertheless converge in a culminating feeling of worry and angst for many participants that persists in a post-9/11 and post-Trump election climate.

My initial foray into understanding the relationships between sound, identity and discrimination began when I was a child watching my father navigate his identity. However, it was not until I began writing and analyzing these experiences that the recent acts of discrimination and violence against immigrants and brown people reaffirmed the harrowing implications of being 'othered'. In many ways, my worry and fear is minor compared to what so many others around the world face, yet, like many of my participants, my anxiety and fear has significantly heightened. Given the rising anti-immigrant and white supremacist political climates - climates which participants made direct reference to in almost all of my interviews, I hope that my dissertation has made a significant contribution 
to breaking down stereotypes, revealing the complexity of identities, and underscoring the multiple forms of discrimination that Indian (and Tamil) Americans experience.

Participant interviews talked in details about the recent political climates of xenophobia and white supremacy in relationship to identity as well as how discrimination and othering exist within Indian and Indian Tamil diasporas. Moreover, popular media, government, and academic homogenization of Indian diaspora communities and legacies of colonialism have long contributed to discriminatory discourses and practices .

\section{Findings}

My research revealed 14 findings about the complexity of identity. These findings were concentrated in five specific areas: 1) Those related to the emergence of hierarchical scalar identities 2) Those related to hybridity and the emergence of postcolonial identities 3) The links between hybridity and scale 4) Those related to sound and identity politics and 5) The ways in which discrimination is multi-layered and contributes to both internal and external politics within the Indian and Indian Tamil diaspora.

\section{Emergence of hierarchical scalar identities}

My first finding was that hierarchical scale was important to how participants conceptualized identities. There are continuing debates about the relevance and usefulness of scale in geography (Haikli, 2018, p. 273). My research, though it complicates the concept of hierarchical scale, demonstrates that hierarchical scale is relevant. As I discussed in Chapter 4, many participants reinforced the fixity of hierarchical scales. In some moments, participants identified with national scales like Indian, while others identified with regional 
scales like Tamil, more local scales like Madurai, or specific villages/cities. As Maari described of himself, "In my heart, I'll always be a guy from Trichy.” When participants identified nationally, regionally, or locally, they suggested a fixed hierarchy in which one identity was separate from the other. An individual had to either identify nationally as Indian or regionally as Tamil. Arvind and Lakshmi's discussions suggested that Indian and Tamil were fixed and could not overlap. Arvind emphasized that Tamil differentiated and counter-narrated the national scale of Indian and to identify as Indian, would undermine identifying specifically as Tamil. Lakshmi suggested that the regional scale of Tamil needed to be deemphasized for the purpose of national unity. In the context of their discussion, neither participant suggested identifying both regionally and nationally - it had to be one or the other.

My second finding builds from my first finding and complicates the static nature of hierarchical scalar identity. Participants often thought about identities as fixed hierarchies, but they also described these scales as multiple (i.e. Indian, Tamil, and American), hybridscaled (Indian-American, Indian-Tamil), or hybrid (the national scale of Indian as resistance to colonial rule). In this way, my participants demonstrated that hierarchical scale was flexible and fluid relying on situation and context (Marston, 2000; Ferber and Harris, 2013). This second finding also highlights two important points: first, as I introduced above - hierarchical scalar identity is simultaneously fluid and hybrid at times, and second, this fluidity often occurred when participants experienced othering (including microaggressions) and discrimination (identified by participants ranging from active hate crimes to feelings of being othered). When participants discussed discrimination or othering, they changed how they identified within a hierarchical scale. For example, in the 
context of US discrimination or othering, some participants who identified as regionally (Tamil) or locally (Trichy), began to identify with national scales like Indian or supranational scales like South Asian or desi. Some even used terms like "brown," 41 suggesting that there was a broader global scale of "brownness" that incorporated South and Southwest Asia and Central and South America.

But, in the context of Indian, Indian American, and Indian Tamil communities, participants often scaled down. As described in Chapter 7, participants who may have identified nationally as Indian, described that they were regionally Tamil in the context of Indian communities. Even Lakshmi, who described herself as Indian, recognized that she was Tamil in Indian communities precisely because she could not speak Hindi and only spoke Tamil. Arvind, Santhya, and others described that the Indian community is dominated by North Indians who often other or discriminate against Tamils based on language or accent. Thus, for them, identifying regionally as Tamil was a way to counternarrate this discrimination. Sometimes, this scaling down was not always purposeful or intentional, but instead a result of active discrimination. Like Sam described, he was denied housing in Mumbai, precisely because others defined him as "a South Indian Tamil."

Even further, within Indian Tamil communities, participants identified with local villages and towns as a reaction to marginalization in Tamil communities in both the US and India. Puran and Pandian both said that other Tamils associated them negative characteristics because of their more local identities within Tamil Nadu - i.e. people from Chennai are greedy or people from Madurai are angry. Participants identifying nationally, subnationally, supranationally, regionally, and locally was contingent upon their

${ }^{41}$ I discuss "brown" later in this chapter. 
perceptions of discrimination or othering within the US (often by other Americans) or within Indian or Indian Tamil communities (often by other Indians or Indian Tamils).

My third finding was Indian and Indian Tamil events and functions used hierarchical scale to purposefully differentiate and dehomogenize Indian identities. For example, events like the CTA used maps and event programs to purposefully distinguish South India from broader India (See Chapter 6). These maps highlighted the region of South India, showing its music as both unique within broader India and also separate from North Indian music. North and South Indian musical tradition differences were also highlighted in speeches and performances. In other words, the CTA represented itself not as Indian, but more specifically as South Indian. CTA events framed identity first and more as subregionally South Indian, and then more locally as Tamil. Much popular representation of Indian music draws from North Indian traditions (Viswanathan and Allen, 2004). Yet, these events emphasized and celebrated Karnatak music's Southern origins. Subnational South Indian identity was recreated throughout the events with language. The lead facilitator spoke English, sometimes using a few Tamil words, but never used Hindi. During performances, most musicians and performers spoke Tamil and much of the audience spoke in conversational Tamil. In this way, the audience recreated this differentiation between North and South India, ultimately disrupting homogenized, national Indian identities.

My fourth finding is that sound - music, accent, and language - reinforced hierarchical scale. Language was one key way that many participants identified as Tamil and accent underlined specific villages or areas in Tamil Nadu. For example, Lakshmi, though she identified nationally as Indian, said that she was Tamil because she spoke Tamil and could not speak or understand Hindi. Pandian, for example, said that his accent reveals 
that he is from Madurai, which he emphasized as part of his local identity. He said that people would know him by the way he talks.

Language, accent as well as music were not just important to participants, but also key to events and performances. For example, NEOTS events emphasized the regional scale of Tamil in multiple ways. The state song of Tamil Nadu took precedence over the national anthems of India and the US. Lyrics and performances were all conducted in Tamil. Songs that were originally in Hindi, were purposefully changed to Tamil. Yet, the national scale of India was not absent. The Indian national anthem was part of the program and most performances or stories took place in India - but India was emphasized through the regional lens of Tamil. For instance, many performance stories that took place in India, took place in Tamil Nadu.

\section{Hybridity - postcolonial identity}

Participants' often referred to their identities in scalar and hierarchical ways, but also demonstrated that these identities were hybrid. My fifth finding is that hybrid identities were often influenced by both colonial binaries and homogenization in the US. Participants like Durga described a hybrid, blurred identity - i.e. neither fully Indian or American, but somewhere in-between these two identities - liminally linking this identity

to both binaries and homogenization. For example, Durga said that she identified as IndianAmerican because she did not have to "explain" her identities. Many participants repeated this sentiment and said that Americans especially like easy answers to "what are you questions" or described that US society prefers "boxed" categories - i.e. either Indian or 
American, not both. Yet, many said their experiences did not fit within these fixed identity categories and thus, they felt somewhere in-between or blurred between these identities.

Some participants linked their in-between, hybrid identities directly to colonial struggle, linking identity to freedom-fighting efforts of family members who resisted colonization. In other words, hybrid identities were born out of colonialism. Lakshmi, for example, tied her identity of being both Indian and Tamil (though she sometimes switched to identifying as one or the other like in the previous example) to her father's experience fighting British colonization in India. If she only identified regionally as Tamil, it undermined his freedom-fighting efforts to unite India against the British. He resisted the British with a uniform national Indian identity. But yet, Lakshmi found that she could not always identify as just Indian, but instead had to be Indian and Tamil. Though colonization in India ended, Lakshmi still carries the remnants of these colonial struggles (that differentiated and binarized Indian/Tamil, British/Indian, West/Non-West) in her identities.

Participants also demonstrated the continuing struggle of still living in a colonial system (Sparke 1998, Gregory, 2004) where colonial binaries like Indian/the West, colonized/colonizer (also adapted and reflected in binaries like immigrant/'citizen,' Indian/American) ${ }^{42}$ persist and shape understandings of the post-colonial world (Gregory, 2004; Radcliffe, 2017; Sparke, 1998). For example, as Radcliffe (2017, p. 24) demonstrates, previous colonial structures of government, citizenship, representation, and knowledge production which separate and categorize, are still deeply embedded in the "colonial present" (including current US society), making colonialism an "ongoing process." She

${ }^{42}$ Many of these categories are linked to whiteness, which I describe in later sections. 
describes how countries like the US ${ }^{43}$ continue to reinforce colonial structures through specific categorizing and representing of groups and people. Homogenization in popular and governmental discourse can reinforce colonial ideas of separation and othering in the US. For instance, like many participants described, Indians are associated with so many stereotypes, many of which do not fit within their personal experiences or views of their own identities. Anusha described frustration with how "people in America can lump me into a category or associate (me with) all these things that have nothing to do with me." Homogenization is one remnant of colonialism that represents "others" in simple, grossly stereotyped forms that emphasize difference from a standard, white norm. Nevertheless, it was important to participant's experiences with their identities.

Hybridity, both as a caveat/challenge to homogenization and colonial binaries and like many participants demonstrated, can create feelings of separation and of loss because authenticity and fixity of identity is unachievable. As Bhabha (1994, p. 119) describes:

It is a 'separate' space, a space of separation - less than one and double - which has been systematically denied by both colonialists and nationalists who have sought authority in the authenticity of 'origins'. It is precisely as a separation from origins and essences that this colonial space is constructed.

While participants demonstrated hybridity in different ways (Lakshmi's identities that still connect to a colonial past, Durga's feeling of always being in-between because

\footnotetext{
${ }^{43}$ This also ties into settler colonialism, something I do not have time to develop in this
} dissertation, but plan to explore in future research. 
"you don't have to explain", or Anusha's resistance to the generalizing of Indian identities in the US) it was nevertheless connected to colonial binaries and homogenization of identity.

\section{The tenuous relationship between scale and hybridity and identity}

My sixth finding related to the relationship between scale and hybridity. This relationship was not clear-cut, but a liminal connection. It was a transitional state, occupying boundaries of scalar and hybrid. Yet, participants often crossed these lines when experiencing and describing their complex identities. My findings illustrate that in some moments, identities were multi-scalar and hierarchical, but in others, they were hybrid. Participants described identities as hierarchical in instances where they felt discrimination or othering, shifting from broader scales like Indian or South Asian with regard to the US to smaller scales like Tamil or Madurai Tamil within Indian or Indian Tamil communities. Events and performances represented identity as hierarchically scalar (Indian, Tamil, South Indian), but simultaneously demonstrated hybridity. For example, the CTA program materials and speeches, though emphasizing the scale of South India, simultaneously highlighted South Indian identity as Cleveland South Indian identity - visibly demonstrating hybrid connections through maps and program guides (see Chapter 6). Furthermore, speeches described that South Indian Karnatak music was now "up to Cleveland to keep the fire burning." In other words, these identities are not fixed within a scalar hierarchy, but quite hybrid at times.

Sometimes, these scales were multiple - existing together and alongside one another (i.e. Tamil and Indian, American and Tamil). Yet, they were also hybrid in that they were in-between, hyphenated, and informed by the leftover politics of colonialism or 
popular homogenization that reinforced colonial binaries. Scale demonstrated nuance yet fixity in identity, often tied to discrimination or othering, while hybridity undercut fixity to show how these identities were theorized as fixed precisely because of both former colonial legacy and broader homogenized narratives in the US, which I discussed in the previous section.

\section{Sound and identity politics}

My seventh finding was that sound - music, accent, or language - could serve as a marker of difference. Sound affected how people thought about, monitored, and even performed identities. For example, many participants felt that if they did not speak Hindi in the larger Indian community, other Indians might ostracize them. In Sam's case, it signified more than just ostracization, because not speaking Hindi and speaking Tamil resulted in being evicted from his house. An "Indian" accent (not sounding American) in the US caused anxiety and fear being in a public setting for many participants. For example, Punniya and Vijaya were uncomfortable leaving their houses, fearing that Americans might make fun of them or even threaten them. Likewise, Matthew attributed his "Indian-sounding" surname, ${ }^{44}$ as a reason his family could not initially find employment in the US. How Americans or even Indians perceived sound had potential to homogenize large groups of people, perpetuate stereotypes, and isolate certain people. For example, the "Indian" accent, Bollywood songs, the Hindi language - were all reasons that many Tamils like Anusha said that they were grouped with things "that had nothing to do with (them)." Thus, participants felt that identifying with 'Indian' music or languages was just a way for white

${ }^{44}$ Not disclosed to protect the anonymity of the participant. 
Americans to identify Tamils as brown, non-white Americans marking them as different or other.

My eight finding was that sound, music, accent, and language, could disrupt both homogenized and traditional (like how NEOTS or CTA represent Indian or Tamil identity) narratives of Indian and Tamil identities, revealing how they are diverse and variegated. For example, some participants broke the stereotypes of Indian and Tamil music. While many participants and organizations like NEOTS or the CTA, associated or represented Tamil identity with Karnatak, filmi ${ }^{45}$, or classical music, Maari thought about Tamil identity as quite different. Instead, he associated his Tamil identity with music from Tamil Nadu that sounded like American 90s grunge bands or Tamil metal bands, breaking stereotypes that 'Indian' or 'Tamil' music are only film or classical songs. Participants like Maari challenged the ways in which traditional Tamil or Indian identities are represented in popular media, academics, governments, and even events and performances. NEOTS or the CTA always include filmi songs or classical songs to represented Indian or Tamil identity in programs, but never such music that Maari described.

My ninth finding was that some participants only recognized othering or discrimination in relation to sound. To elaborate, initially, some participants denied that they had experienced discrimination. Yet, when I brought up accent or language, they began to describe experiences of othering and discrimination. For example, participants like Punniya said that she never felt discriminated against in the US. Yet, when I asked about language or accent, she subsequently described fears of going out in public precisely

\footnotetext{
${ }^{45}$ A term that refers to "of films" in Indian cinema.
} 
because her accent. She feared people might make fun of her or even threaten her. This prevented from leaving her house except to go out to the hospital for her volunteer work.

My tenth finding was that language, music, and accent were significant to discrimination and affected how some participants navigated their lives in the US. Accent was a marker of difference in the US that participants felt many Americans used to designate them as "other" or actively discriminate against them. Speaking Tamil or any Indian language within the US would bring attention to them as 'other', brown, or foreigner. Anusha describes how she feels angst when her parents speak Tamil in airports because it makes them stand out - they look and sound "brown." Vimala describes how a store cashier harassed her parents by pretending that she did not understand them, laughing, and making Vimala's mother repeat herself over and over. Ruth describes how cashiers in Target would not even acknowledge or speak to her because of her accent. Some participants said that when someone (often a white American) brought attention to their accent, it became more pronounced, making them feel extremely uncomfortable. Participants also that Americans had a double standard - Indians were required to 'fix' their accents, but white Americans could butcher the pronunciation of Indian names with no consequences.

Meanwhile, in Indian communities, some participants mentioned that they were ridiculed for not speaking Hindi or described with derogatory terms like "Madrasi." Speaking Tamil in Indian communities marked them as different, less-cultured, or even stubborn, or backward. In Tamil communities, accent designated someone with certain characteristics - like cosmopolitanism, laziness, greediness (a Chennai accent), violence (a Madurai accent), or others. It intersected with caste, marking some as Brahmin and others 
as non-Brahmin, i.e. with the use of words like "jalum" (Brahmin word for water) instead of "thanni" (non-Brahmin word for water. Music, like Karnatak music, was described as 'Tamil' by many participants and used in many events and programs. However, some participants said that the use of this music represented Tamil identity as upper caste and Brahmin and discriminated against local folk music and local rural Tamil identities.

My eleventh finding was that participants linked environmental sounds to memories, emotions, and a multi-sensory experience. Sounds like spices popping in a pan, pouring of tea, and whistles of a pressure cooker reminded participants of memories with family members sharing food or cooking in the kitchen. These sounds also invoked feelings of happiness, sadness, loss, or nostalgia - i.e. thinking about the memories of sharing food with a loved one who had since passed. In some instances, participants connected the sounds of food to a broader multi-sensory experience that included smell and taste - i.e. described by multiple participants was that the sound of boiling tea led them to imagine the taste of tea. The sound of a pressure cooking led them to imagine the smells of Indian spices.

Overall, sound was pervasive to participants' experiences of identity manifesting through music, accent, language, and environmental sounds. Many times, sound linked to discrimination or othering. However, sometimes, sound, especially environmental sounds, linked more closely to feelings and memories.

\section{Multi-level discrimination, othering, and identity}

My twelfth finding was that, unsurprisingly, fears of discrimination and othering, from microaggressions to active hate crimes, were heightened for many participants in 
current political climates. Many had experienced othering or active hate crimes in the past, and after the election of Donald Trump, began to fear the reemergence of these threats. Participants described that they felt that xenophobia, white supremacy, and Islamophobia were heightened after Trump's election. Some referenced specific incidents like the 2017 travel ban or the shooting of Srinivas Kuchibholta in Kansas. These participants, though they had not directly experienced hate crimes during the Trump administration, nevertheless, had concerns that active hate crimes could and would happen to them. For some participants, this fear manifested emotionally, psychologically, and sometimes even physically. For example, quite a few participants said that they felt physically ill (nausea, faintness, panic) after the election or when they heard about some new "horrible thing" Trump had done. Bharathi said that she developed insomnia as well as an inability to leave her house comfortably (worrying that someone would say or do something to her). As Joshi, McCutcheon, and Sweet (2015) have argued, physical effects of microaggressions and discrimination (i.e. fear or anxiety) change the way that people of color operate in their daily lives (avoiding certain situations, areas, confrontations). Just as microaggressions serve as institutional white supremacy that disadvantages and psychologically affects people of color in all areas of American life, (Joshi, McCutcheon, \& Sweet, 2015), so, for many participants, did fear of discrimination or othering.

My thirteenth finding was that participants viewed geographical location as significant to their experiences with discrimination and othering. Many said that they were more likely to experience discrimination or othering in rural or "red" areas than cosmopolitan, diverse areas. A few participants described these diverse areas as generally concentrated on the "coasts". For example, Fathima said that she had experienced more 
instances where people stared at her or threatened her in rural areas that she characterized as "internal, red states (referring to political leaning of conservative or Republican)." Preeti described these areas (where she had experienced stares and threats in rural Pennsylvania) as "rural, confederate flag-waving areas." Discrimination was also associated with the US South. Maari described incidents where he was othered in Florida and Texas, Nazeem described how people in Texas were secretly racist to her, and Matthew described active discrimination, based on his surname (along with other incidents) in Alabama. Most participants agreed that they were more likely to experience discrimination or othering in rural or Southern areas. Many scholars have noted before that areas with less diversity, especially rural areas, are more likely to support anti-immigration and xenophobic views (Chacón \& Davis, 2018; Fennelly \& Federico, 2008). In these areas, scholars have noted that non-white, visibly, audibly, and culturally, residents stand out in homogenous areas. Not surprisingly, many participants associated rural areas with whiteness and white culture because when they were in those spaces, they noticed that they became the visible and audible minority. Although many participants described discrimination in rural areas, discrimination was not limited to these areas and happened even in urban areas. Participants still described fears of discrimination in college towns and large urban centers, though fewer described active hate crimes. Many participants viewed coastal areas, or larger, more diverse areas - like urban centers - as safer because "diversity" was more likely. In other words, participants viewed areas that supported a diverse population as safer than rural or "red" areas.

My fourteenth finding was that the Indian and Indian Tamil diaspora mimicked/replicated the discrimination and othering that many Indians or Indian Tamils 
experienced in the broader US or that their families experienced during colonial occupation. Indian communities in the US primarily replicated discrimination and othering through ideas of whiteness and anti-blackness, often remnants of colonial practice.

Colonizers used concepts of race (whiteness and blackness) to subdue and subordinate colonial subjects (Fanon, 1967; 1963). Bhabha (1994) suggests that the diasporic subject, a product of colonization, is still subject to these categories of race, striving to achieve the privileges of whiteness, but never fully attaining them. Indians mimic white Americans, even if only reaping some of the benefits of being white (because they still experience racism and discrimination). The Indian subject within the US is a "subject of difference that is the same, but not quite..." (Bhabha, 1994, p. 86). The Indian subject imitates whiteness that reinforces racial power structures. For example, participants described that Indians tend to identify with specific qualities like economic standing, profession, political opinions, command of English - that made them more deserving of their place in American society than other immigrant groups. Many of their descriptions of these qualities fall under the category of the "social condition(s) of whiteness" (Bonds and Inwood, 2016, 719), perpetuating whiteness and racism within Indian communities.

Scholars like Bhatia (2007) have suggested that Indians in the US tend to identify more with white communities to actively distance themselves from communities of color and maintain what he calls "brown privilege", or a subdued version of white privilege. For example, many participants described experiences where family members or relatives looked down on other communities of color because they felt that they were in a better position that these communities. These friends or family, participants said, would use their 
economic position or command of the English language to justify that they somehow earned their place and privilege within American society. Santhya or Sakthi, for example, described how their family or friends had often made many anti-black remarks, specifically directed at black Americans.

Whiteness and racism in Indian communities also applied to other Indians (not just other communities of color). For example, some participants felt that North Indian communities were privileged because they were whiter than South Indians. Some described that this was heightened by colonial rule that prefaced whiteness over darkness. To the colonizers, Indians, were dark savages, "backward", and the opposite of the white, civilized colonizer (Seth, 2010). Some participants said that in India and Indian diaspora communities, Tamils were associated with blackness and backwardness. In many ways, this related to the leftover politics of colonialism, such as how North Indians would call South Indians "Madrasi" - a derogatory term used by the British to describe South Indians.

In the Indian and Indian Tamil diaspora, whiteness also maintained a liminal link to caste and religion. Some participants associated higher castes with more with privilege and whiteness. For example, some participants said that Brahmins were often lighter-skinned than non-Brahmins and they had more economic wealth. Other participants centered on concepts like Aryan (from the North) and Dravidian (from the South) to describe that darkskinned Tamils were often non-Brahmin Dravidians that were othered and even silenced by the Brahmin (potentially-Aryan - even if they were Tamil) community. Many participants (Hindu, Muslim, and Christian), mentioned that they felt that Indian communities overall try to distance themselves from Islam or anything that would deem them as an "other" to a white, Christian society. Islam was a marker of "brownness" or non- 
whiteness that separated Indians from maintaining positions of privilege in US society. Overall, whiteness was pervasive, not just in US society, but also within both the Indian and Indian Tamil diaspora.

\section{Future Work}

In the process of conducting research, I also identified additional concepts or factors that need further examination and inquiry. These include geographical location, religion, gender, sexuality, and caste. Participants identified geographical location, particularly rural areas, as places that they were more likely to experience discrimination or othering. A more robust analysis with a variety of demographic groups and a larger sample could shed additional insights on the relationship between geographical location and discrimination and othering.

Factors like religion and caste, though they were not the focus of my research, influenced participant responses and ways that they thought about identity, discrimination, and othering. While I only touched on them briefly, I could further develop the links between religion, caste, and whiteness in a follow-up study.

Gender and sexuality also informed participant identities. These were not always the first factors participants mentioned regarding their identities, but they were often connected to their identities. For example, some participants identified as heterosexual (without being asked about sexuality), almost as a statement to let me know that they were not homosexual (sometimes suggesting negative connotations of homosexuality) ${ }^{46}$. Other

\footnotetext{
${ }^{46}$ Though, some would follow-up with "not that there is anything wrong with that."
} 
participants who identified as queer, briefly alluded to the difficult experiences of identifying as queer within the South Asian community - a very heteronormative, cisnormative, and gender-binary community. Participants like Nazeem (and many others) described the difficulties with what they described as South Asian gender roles (i.e. a woman needing to find a husband, have children, or carry on cultural traditions) ${ }^{47}$. In future research, I want to address these factors which were important to contextualizing many participants' identities.

Lastly, I also plan to examine the links between the Sri Lankan Tamil diaspora and the Indian Tamil diaspora, which is not discussed in this research. I have already started preliminary interviews on this project.

\section{Delimitations and Limitations}

I focused on Indian Tamils in the US and more specifically, Indian Tamils connected to Northeast Ohio, northern New Jersey, and Morgantown, WV. I did so because these sites reflected thee different sizes of Tamil communities ranging in size from medium, large, to small (respectively). I also limited my focus to Indian Tamils rather than the broader Indian community in order to bring attention nuance within the Indian diaspora while also shedding light on an understudied subgroup.

First, as mentioned above, I have a lack of discussion on gender and sexuality. These are important to understanding discrimination, marginalization, or even homogenization of identities within communities. The breadth and scope of this study did not adequately

\footnotetext{
47 Though some also pointed out the irony in those same gender roles in conservative
} Christian communities in the US. 
address these factors but did lay the groundwork for future research regarding these factors.

Second, I discovered Critical Race Theory (CRT) towards the end of my research and would have liked to develop my work more directly in connection with this literature. I intend on developing the links of CRT and postcolonialism

A third area in need of further development was with respect to postcolonialism and settler colonialism. As much of my research takes place in the United States, I feel that my future work will need to discuss the implications of a postcolonial diaspora in a settler colonial state. I did not address settler colonialism because this was not something I uncovered until the latter stages of the dissertation and it has been left somewhat undeveloped.

A fourth limitation relates to the Introduction where I discuss the importance of representation and discourse analysis formed by government, media, and academics. I did not analyze such representations, but instead used them as a way to identify my problem of homogenization. In future work, I hope to examine representational discourses more.

A fifth limitation is the breadth and depth of my sample. I was able to interview 55 individuals, but only 39 of these interviews met my qualifications for NVivo analysis. My methods allowed me to conduct in-depth, detailed interviews - some lasting almost 3 hours. In my proposal, I discussed using a survey to obtain broader samples. However, after administering 2 or 3 of these, I found that these surveys and questionnaires were much too superficial. For example, explaining complex concepts like sound or identity require a two-way dialogue between the researcher and participants. Additionally, I felt that the questions on the survey repeated much of the data that I was gathering in my 
interviews. A survey would have created a larger sample size, but 55 interviews provided the depth I was seeking.

\section{Concluding thoughts}

Identities are multi-layered, hierarchical, hybrid, complex, and influenced by a variety of factors. Many of these factors - sound, hierarchical scale, and hybridity - are also connected to discrimination and othering. As Dyer (1997), Bonds and Inwood (2016), Joshi, McCutcheon, and Sweet (2015) and many other CRT scholars suggest, whiteness is pervasive and threaded in all aspects of society (economics, politics, institutions, media, governments, and everyday interactions). Whiteness is heightened in current political climates, dominating political rhetoric, institutional administration, and everyday interactions for people of color (Gökarıksel \& Smith, 2016; Joshi et al., 2015). Much academic and activist research on discrimination and Indian communities revolves around discrimination onto these communities. But as I've demonstrated in my research, whiteness pervades into the Indian diaspora in the US and discrimination is both experienced and mimicked by Indians within Indian communities. Mimicry of whiteness and racism in Indian communities contributes to pervasive issues of whiteness in broader US society.

By identifying nuance of identity through scale and hybridity and underscoring the importance of sound in identity and discrimination, I highlight some links between homogenization, whiteness, and colonialism. Whiteness is inextricably linked to colonialism and colonialism is embedded in our ways of knowing and understanding the world. Sparke (1998) has noted that current societies cannot escape colonialism precisely 
because they are trapped within colonial structures and ways of thinking. The first step to changing colonial structures is to recognize them.

I hope my dissertation becomes part of the process of changing a discourse that affects (even in subtle ways) so many Indians and people of color in the US. I believe that this process can happen by revealing the richness of identity and the subtle and overt forms of discrimination that are much too common. After completing this dissertation, I plan to publish articles in academic journals detailing the importance of recognizing nuance in Indian diasporas as well as pointing to the ways that whiteness is mimicked within these diasporas. I also plan to distribute an executive summary of my findings to my participants, especially to those who expressed interest in a follow-up discussion of the results.

Current homogenization in academia and media representations of identities masks the many pervasive issues of whiteness that are nuanced and layered. I am not suggesting that homogenization is not useful for communities to build solidarity (as demonstrated through participants identifying with broader scales or identities like Indian, desi, or South Asian). Instead, I hope for greater action and awareness that (even slowly) accomplishes three goals: 1) (de)homogenizes identities and discussions of othering and discrimination in academic and media circles, 2) decolonizes academic, governmental, and popular approaches to identity, and 3) advocates for a future that recognizes, and values marginalized groups (not just Indians), giving them true equality. I hope that my dissertation becomes a part of making these goals a reality. 


\section{References}

Aitken, S. (2010). “Throwntogetherness": Encounters with Difference and Diversity. In D.

DeLyser, S. Aitken, S. Herbert, M. Crang, \& L. McDowell (Eds.), Sage Handbook of Qualitative Geography (pp. 46-69). London: SAGE.

Alessandrini, A. C. (2001). "My Heart's Indian for All That": Bollywood Film between Home and Diaspora. Diaspora, 10(3), 315-339.

Anderson, B. (2009a). Affective atmospheres. Emotion, Space and Society, 2(2), 77-81.

https://doi.org/10.1016/j.emospa.2009.08.005

Anderson, B. (2009b). Non-Representational Theory. In D. Gregory, R. Johnston, G. Pratt, M. J. Watts, \& S. Whatmore (Eds.), The Dictionary of Human Geography. Malden, MA: Wiley Blackwell.

Anderson, B., \& McFarlane, C. (2011). Assemblage and geography. Area, 43(2), 124-127. https://doi.org/10.1111/j.1475-4762.2011.01004.x

Anderson, B., Morton, F., \& Revill, G. (2005). Practices of music and sound. Social \& Cultural Geography, 6(5), 639-644. https://doi.org/10.1080/14649360500298282

Anderson, K. (1987). The Idea of Chinatown: The Power of Place and Institutional Practice in the Making of a Racial Category. Annals of the Association of American Geographers, 77(4), 580-598. https://doi.org/10.1111/j.1467-8306.1987.tb00182.x

Anderson, K., \& Smith, S. J. (2001). Editorial: Emotional geographies. Transactions of the Institute of British Geographers, 26(1), 710. https://doi.org/10.1111/1475-5661.00002 Antonsich, M. (2018). Living in diversity: Going beyond the local/national divide. Political Geography, 63, 1-9. 
Anzaldúa, G. (1987). Borderlands/La Frontera: The New Mestiza. San Francisco: Aunt Lute Books.

Arnold, D. (2010). British India and the "beriberi problem", 1798-1942. Medical History, 54(3), 295-314.

Attali, J. (1985). Noise: The political economy of music (Vol. 12). Manchester University Press Vol. 16.

Bandyopadhyay, R., \& Morais, D. (2005). Representative dissonance: India’s self and western image. Annals of Tourism Research, 32(4), 1006-1021.

Barrett, K. (2018). Asian-American and Pacific Islander Heritage Month: May 2018.

Bate, B. (2009). Tamil Oratory and The Dravidian Aesthetic: Democratic Practice in South India. New York: Columbia University Press.

Besio, K. (2003). Steppin'in it: postcoloniality in northern Pakistan. Area, 35(1), 24-33.

Bhabha, H. K. (1994). The Location of Culture. London: Routledge.

Bhabha, H. K. (2015). Foreword. In P. Werbner \& T. Modood (Eds.), Debating Cultural Hybridity: Multicultural Identities and the Politics of Anti-Racism (New, pp. ix-xiv). London: Zed Books.

Bhardwaj, S., \& Rao, N. M. (1990). Asian Indians in the United States: a geographic appraisal. In C. Clarke, C. Peach, \& S. Vertovec (Eds.), South Asians Overseas: Migration and Ethnicity (pp. 197-217). Cambridge: Cambridge University Press.

Bhat, C., \& Narayan, K. . (2010). Indian Diaspora, Globalization and Transnational Networks: The South African Context. Journal of Social Science, 2, 13-23.

Bhatia, S. (2007). American karma: Race, culture, and identity in the Indian diaspora. NYU Press. https://doi.org/10.1007/s13398-014-0173-7.2 
Blunt, A. (2007). Cultural geographies of migration: mobility, transnationality and diaspora. Progress in Human Geography, 31(5), 684-694. https://doi.org/10.1177/0309132507078945

Bobo, L. D. (2017). Racism in Trump's America: reflections on culture, sociology, and the 2016 US presidential election. British Journal of Sociology, 68, S85-S104. https://doi.org/10.1111/1468-4446.12324

Bonds, A., \& Inwood, J. (2016). Beyond white privilege. Progress in Human Geography, 40(6), 715-733. https://doi.org/10.1177/0309132515613166

Bose, P. (2018). Indian Diasporas and the Creation of Subnational Identities. In D. H. Kaplan \& G. H. Herb (Eds.), Scaling Identities: Nationalism and Territoriality (pp. 255-271). Lanham, Md.: Rowman and Littlefield.

Braziel, J. E. (2008). Diaspora: An Introduction. Oxford: Blackwell Publishing Ltd. Brenner, N. (2004). New State Spaces: Urban Governance and the Rescaling of Statehood. Oxford: Oxford University Press.

Brubaker, R. (2005). The 'diaspora' diaspora. Ethnic and Racial Studies, 28(1), 1-19. https://doi.org/10.1080/0141987042000289997

Brunea, M. (2010). Diaspora, transnational spaces and communities. In R. Bauböck \& T. Faist (Eds.), Diaspora and Transnationalism : Concepts, Theories and Methods (pp. 35-50). Amsterdam: Amsterdam University Press.

Carles, J. L., Barrio, I. L., \& De Lucio, J. V. (1999). Sound influence on landscape values. Landscape and Urban Planning, 43(4), 191-200. https://doi.org/10.1016/S01692046(98)00112-1

Carter, R. T. (2007). Racism and Psychological and Emotional Injury: Recognizing and 
Assessing Race-Based Traumatic Stress. The Counseling Psychologist, 35(1), 13-105. https://doi.org/10.1177/0011000006292033

Carter, R. T., Sant-barket, S. M., Carter, R. T., \& Sant-barket, S. M. (2014). Traumatology Assessment of the Impact of Racial Discrimination and Racism : How to Use the RaceBased Traumatic Stress Symptom Scale in Practice Assessment of the Impact of Racial Discrimination and Racism : How to Use the Race-Based Traumatic Stress Symp, 21(1), $32-39$.

Chacko, E., \& Menon, R. (2013). Longings and belongings: Indian American youth identity, folk dance competitions, and the construction of "tradition." Ethnic and Racial Studies, 36(1), 97-116. https://doi.org/10.1080/01419870.2011.634504

Chacón, J. A., \& Davis, M. (2018). No one is illegal: Fighting racism and state violence on the US-Mexico border (2nd ed.). Chicago: Haymarket Books.

Chand, M., \& Tung, R. L. (2014). Bicultural identity and economic engagement: An exploratory study of the Indian diaspora in North America. Asia Pacific Journal of Management, 31(3), $763-788$.

Chidambaram, M. (1987). Cultural Entrepreneurs and Language Strategists: DMK In Tamil Nadu. The Indian Journal Of Political Science, 418-427.

Chishti, M., Hipsman, F., \& Ball, I. (2015). Fifty Years On, the 1965 Immigration and Nationality Act Continues to Reshape the United States. Washington DC.

Chou, R. S., \& Feagin, J. R. (2015). Myth of the Model Minority: Asian Americans Facing Racism. Routledge.

CNN. (2013, September 17). Miss America crowns 1st winner of Indian descent. Cable Network News, p. 1. Retrieved from https://www.cnn.com/2013/09/16/showbiz/miss-america-racist- 
reactions/index.html

Coates, T.-N. (2017). The First White President. The Atlantic. Retrieved from https://www.theatlantic.com/magazine/archive/2017/10/the-first-white-president-ta-nehisicoates $/ 537909 /$

Cohen, R. (1997). Global diasporas : an introduction. Seattle: University of Washington Press.

COI. (n.d.). Comparative rankings of Scheduled Languages in descending order of speaker's strength-1971, 1981, 1991 and 2001. New Delhi. Retrieved from http://www.censusindia.gov.in/Census_Data_2001/Census_Data_Online/Language/Stateme nt1.aspx

CTA. (n.d.). Website.

Culcasi, K. (2011). Cartographies of supranationalism: Creating and silencing territories in the “Arab Homeland.” Political Geography, 30(8), 417-428.

Dave, S. S. (2013). Indian accents: Brown voice and racial performance in American television and film. Champaign: University of Illinois Press.

Davidson, J., \& Milligan, C. (2004). Embodying emotion sensing space: introducing emotional geographies. Social \& Cultural Geography, 5(4), 523-532. https://doi.org/10.1080/1464936042000317677

De Certeau, M. (1984). The Practice of Everyday Life. (S. F. Rendall, Ed.), Practice.

Delgado, R., \& Stefancic, J. (2017). Critical race theory: An introduction (Third). New York: NYU Press.

Desai, J. (2004). Beyond Bollywood. New York: Routledge.

Devadas, Vijay and Velayutham, S. (2008). Encounters with "India": Ethnonationalism in Tamil cinema. In S. Velayutham (Ed.), Tamil Cinema: the cultural politics of India's other film 
industry.

Devadoss, C. (2017). Sound and identity explored through the Indian Tamil diaspora and Tamil Nadu. Journal of Cultural Geography, 34(1), 70-92.

https://doi.org/10.1080/08873631.2016.1231383

Dewsbury, J.-D. (2010). Language and the Event: The Unthought of Appearing Worlds. In B. Anderson \& P. Harrison (Eds.), Taking-Place: Non-Representational Theories and Geography (pp. 147-160). Farnham: Ashgate. Retrieved from http://dro.dur.ac.uk/6946/

Dickinson, J., \& Bailey, A. J. (2007). (Re)membering diaspora: Uneven geographies of Indian dual citizenship. Political Geography, 26(7), 757-774.

https://doi.org/10.1016/j.polgeo.2007.03.007

Doel, M. (2010). Representation and Difference. In B. Anderson \& P. Harrison (Eds.), Taking Place: Non-representational theories and geography (pp. 117-137). Farnham: Ashgate. https://doi.org/10.2307/25563511

Dowling, R. (2010). Power, Subjectivity and Ethics in Qualitative Research. In Iain Hay (Ed.), Qualitative Research Methods in Human Geography (pp. 23-37). Don MIlls, ON: Oxford University Press.

Dufoix, S. (2008). Diasporas. Berkeley: University of California Press. Retrieved from http://site.ebrary.com/id/10229946

Dunn, K. (2010a). Embodied transnationalism: Bodies in transnational spaces. Population, Space and Place, 16(1), 1-9. https://doi.org/10.1002/psp.593

Dunn, K. (2010b). Interviewing. In I. Hay (Ed.), Qualitative Research Methods in Human Geography (Third Edit, pp. 101-138). Don MIlls, ON: Oxford University Press.

Dyer, R. (1997). White. London: Routledge. 
Eck, D. (2018). Asians and Asian Exclusion. The Pluralism Project. Cambridge. Retrieved from http://pluralism.org/encounter/historical-perspectives/asians-and-asian-exclusion/

Edney, M. (1997). Mapping an empire: the geographical construction of British India 17651843. Chicago and London: University of Chicago Press.

Faist, T. (2010). Diaspora and transnationalism: What kind of dance partners? In R. Bauböck \& T. Faist (Eds.), Diaspora and Transnationalism : Concepts, Theories and Methods (pp. 934). Amsterdam: Amsterdam University Press.

Fanon, F. (1963). The Wretched of the Earth. (Translated by C. Farrington, Ed.). New York: Grove Press.

Fanon, F. (1967). Black skin, white masks [1952]. New York: Grove Press.

Farina, G. (2014). Some refl ections on the phenomenological method. DIAL PHIL MENT NEURO SC, 50-62. Retrieved from www.crossingdialogues.com/journal.htm

Fennelly, K., \& Federico, C. (2008). Rural residence as a determinant of attitudes toward US immigration policy. International Migration, 46(1), 151-190. https://doi.org/10.1111/j.1468-2435.2008.00440.x

Ferber, M. P., \& Harris, T. M. (2011). Resurrecting Scale in Emergent Geography of Religion. International Journal of Humanities and Social Science, 1(12), 1-9.

Ferber, M. P., \& Harris, T. M. (2013). Critical Realism and Emergence in a Scaled Geography of Religion. Journal of Critical Realism, 2(12), 183-201.

Franklin, A. J., Boyd-Franklin, N., \& Kelly, S. (2006). Racism and Invisibilty: Race-Related Stress, Emotional Abuse and Psychological Trauma for People of Color. Journal of Emotional Abuse, 6, 9-30. https://doi.org/10.1300/J135v6n02

Gallagher, M., Kanngieser, A., \& Prior, J. (2016). Listening geographies. Progress in Human 
Geography, 41(5), 030913251665295. https://doi.org/10.1177/0309132516652952

Ganti, T. (2013). Bollywood: a guidebook to popular Hindi cinema. Routledge.

Gems, G. R. (2006). Sport, Colonialism, and United States Imperialism. Journal of Sport History, 33(1), 3-25.

Gibson, M. A. (1988). Punjabi orchard farmers: an immigrant enclave in rural California. The International Migration Review, 22(1), 28-50. Retrieved from http://ovidsp.ovid.com/ovidweb.cgi?T=JS\&PAGE=reference\&D=med3\&NEWS=N\&AN=1 2281049

Gilmartin, M., \& Berg, L. D. (2007). Locating postcolonialism. Area, 39(1), 120-124. https://doi.org/10.1111/j.1475-4762.2007.00724.x

Gilroy, P. (1993). The Black Atlantic Modernity and Double-Consciousness. Cambridge: Harvard University Press.

Glass, I., \& Meek, M. (2017). Our Town - Part One. United States: WBEZ Chicago. Retrieved from https://www.thisamericanlife.org/632/our-town-part-one

Gökarıksel, B., \& Smith, S. (2016). "Making America great again"?: The fascist body politics of Donald Trump. Political Geography, 54, 79-81.

https://doi.org/10.1016/j.polgeo.2016.07.004

Gregory, D. (2004). The Colonial Present. Malden, MA: Blackwell Publishing Ltd. Retrieved from https://books.google.com/books?id=DejCbO1mvCYC

Grewal, I., \& Kaplan, C. (1994). Scattered hegemonies: postmodernity and transnational feminist practices. In I. Grewal \& C. Kaplan (Eds.) (pp. 1-37).

Grosz, E. (2008). Vibration, Animal, Sex, Music. In Chaos, territory, art: Deleuze and the framing of the earth (pp. 25-63). New York: Columbia University Press. 
Groth, P. (1997). Frameworks for Cultural Landscape Study. In P. E. Groth \& T. W. Bressi (Eds.), Understanding Ordinary Landscapes (pp. 1-25). New Haven: Yale University Press.

Häkli, J. (2018). Afterword: Transcending Scale? In D. H. Kaplan \& G. H. Herb (Eds.), Scaling Identities: Nationalism and Territoriality (pp. 271-283). Lanham, Md.: Rowman and Littlefield.

Haldrup, M., Koefoed, L., \& Simonsen, K. (2006). Practical orientalism--bodies, everyday life and the construction of otherness. Geografiska Annaler: Series B, Human Geography, 88(2), 173-184. https://doi.org/10.1111/j.0435-3684.2006.00213.x

Hall, S. (1996). Ethnicity: Identity and Difference. In R. G. Eley, Geoff and Suny (Ed.), Becoming national: a reader (pp. 339-349). New York: Oxford University Press.

Hall, S. (2003). Cultural Identity and Diaspora. In J. E. Braziel \& A. Mannur (Eds.), Theorizing Diaspora: A Reader (pp. 233-247). Malden, MA: Theorizing Diaspora: A Reader.

Harris, C. (2004). "How Did Colonialism Dispossess? Comments from an Edge of Empire,.” Annals of the Association of American Geographers, 94(1), 165-182.

Harris, L. M. (2014). Imaginative Geographies of Green: Difference, Postcoloniality, and Affect in Environmental Narratives in Contemporary Turkey. Annals of the Association of American Geographers, 104(4), 801-815. https://doi.org/10.1080/00045608.2014.892356

Harrison, P. (2010). Testimony and the Truth of the other. In B. Anderson \& P. Harrison (Eds.), Taking Place: Non-representational theories and geography (pp. 161-179). Farnham: Ashgate.

Hartman, S. (2008). Lose Your Mother: A Journey Along the Atlantic Slave Route. New York: Farrar, Straus and Giroux. 
Herb, G. H. (2018). Power, Territory, and National Identity. In G. H. Herb \& D. H. Kaplan (Eds.), Scaling Identities: Nationalism and Territoriality (pp. 7-31). Lanham: Rowman and Littlefield.

Herod, A., \& Wright, M. W. (2002). Placing scale: an introduction. In A. Herod \& M. W. Wright (Eds.), Geographies of power: Placing scale (pp. 1-14). Malden, MA: Blackwell Publishing Ltd.

HLCID. (2001). Report of the High Level Committee on The Indian Diaspora. New Delhi.

Hoeffel, E. M., Rastogi, S., Kim, M. O., \& Shahid, H. (2012). The Asian Population: 2010.

Hopkins, P., Botterill, K., Sanghera, G., \& Arshad, R. (2017). Encountering Misrecognition: Being Mistaken for Being Muslim. Annals of the American Association of Geographers, 107(4), 934-948. https://doi.org/10.1080/24694452.2016.1270192

Hyndman, J. (2012). The geopolitics of migration and mobility. Geopolitics, 17(2), 243-255. https://doi.org/10.1080/14650045.2011.569321

Ifekwunigwe, J. O. (2003). Returning (s): Relocating the critical feminist auto-ethnographer. In J. E. Braziel \& A. Mannur (Eds.), Theorizing Diaspora: A Reader. Oxford: Blackwell. Inwood, J., \& Bonds, A. (2016). Confronting white supremacy and a militaristic pedagogy in the U.S. settler colonial state. Annals of the American Association of Geographers, 106(3), 521-529. https://doi.org/10.1080/24694452.2016.1145510

Iyer, S. H. S. R. D. (2014). Under Suspicion, Under Attack: Xenophobic Political Rhetoric and Hate Violence against South Asian, Muslim, Sikh, Hindu, Middle Eastern, and Arab Communities in the United States.

Jacob, P. (2009). Celluloid Deities: The Visual Culture of Cinema and Politics in South India. Lanham: Lexington Books. 
Jain, S. (2014). Spiritual India: restoring the white savior with purpose. Dialogue Humanities Review, 1,91 .

Jansson, D. (2010). Racialization and “Southern” Identities of Resistance: A Psychogeography of Internal Orientalism in the United States. Annals of the Association of American Geographers, 100(1), 202-221. https://doi.org/10.1080/00045600903379109

Jayaram, N. (2010). Revisiting the City : The Relevance of Urban Sociology Today. Economic And Political Weekly, XLV(August 2009), 50-57.

Jazeel, T. (2005). The world is sound? Geography, musicology and British-Asian soundscapes. Area, 37(3), 233-241. https://doi.org/10.1111/j.1475-4762.2005.00626.x

Jazeel, T. (2006). Postcolonial geographies of privilege: Diaspora space, the politics of personhood and the "Sri Lankan Women's Association in the UK." Transactions of the Institute of British Geographers, 31(1), 19-33. https://doi.org/10.1111/j.14755661.2006.00192.x

Jenkins, K. (2007). The Best of Multimedia Photojournalism: The Era of the Ear. Retrieved May 3, 2013, from https:/www.poynter.org/2007/the-best-of-multimedia-photojournalism-theera-of-the-ear/81493/

Johnson, C., \& Coleman, A. (2012). The Internal Other: Exploring the Dialectical Relationship Between Regional Exclusion and the Construction of National Identity. Annals of the Association of American Geographers, 102(4), 863-880. https://doi.org/10.1080/00045608.2011.602934

Jones, L. D. R. (2001). Geographies of languages/Languages of geography. Social \& Cultural Geography, 2(3), 261-264. https://doi.org/10.1080/14649360121793

Jones, R. (2012). Border Walls: Security and the War on Terror in the United States, India, and 
Israel. London/New York: Zed Books.

Jones, R. (2016). Violent borders: Refugees and the right to move. London: Verso Books.

Jones, R., \& Merriman, P. (2009). Hot, banal and everyday nationalism: Bilingual road signs in Wales. Political Geography, 28(3), 164-173. https://doi.org/10.1016/j.polgeo.2009.03.002

Joshi, S., McCutcheon, P., \& Sweet, E. L. (2015). Visceral geographies of whiteness and invisible microaggressions. Acme, 14(1), 298-323.

Kalidos, R. (1976). History and culture of the Tamils: from prehistoric times to president's rule. Dindigul: Vijay.

Kalra, A., \& Asokan, S. (2014, June 19). Modi's push for Hindi struggles to translate in some states. Reuters, p. 1. Retrieved from https://in.reuters.com/article/india-language-hindmodi/modis-push-for-hindi-struggles-to-translate-in-some-statesidINKBN0EU1B720140619

Kalra, V., Kaur, R., \& Hutnyk, J. (2005). Diaspora and Hybridity. London: SAGE.

Kanngieser, A. (2012). A sonic geography of voice: Towards an affective politics. Progress in Human Geography, 36(3), 336-353. https://doi.org/10.1177/0309132511423969

Kapchan, D. (2016). Slow Activism: Listening to the Pain and Praise of Others. International Journal of Middle East Studies, 48(01), 115-119.

https://doi.org/10.1017/S0020743815001506

Kaplan, D. H. (2018). National Identity and Scalar Processes. In G. H. Herb \& D. H. Kaplan (Eds.), Scaling Identities: Nationalism and Territoriality (pp. 31-49). Lanham: Rowman and Littlefield.

Kaplan, D. H., \& Herb, G. H. (2018). Introduction: Scaling the Nation. In D. H. Kaplan \& G. H. Herb (Eds.), Scaling Identities: Nationalism and Territoriality (pp. 1-5). Lanham, Md.: 


\section{Rowman and Littlefield.}

Kearns, R. (2010). Knowing seeing? Undertaking observational research. In I. Hay (Ed.), Qualitative Research Methods in Human Geography (Third, pp. 241-259). Don MIlls, ON: Oxford University Press.

Keith, M., \& Pile, S. (2004). Place and the Politics of Identity. Routledge.

Kishi, K. (2017). Assaults against Muslims in U.S. surpass 2001 level. Washington DC. Retrieved from http:/www.pewresearch.org/fact-tank/2017/11/15/assaults-against-muslimsin-u-s-surpass-2001-level/

Kobayashi, A., \& Peake, L. (2000). Racism out of Place: Thoughts on Whiteness and an Antiracist Geography in the New Millennium. Annals of the Association of American Geographers, 90(2), 392-403.

Kompridis, N. (2005). Normativizing hybridity/neutralizing culture. Political Theory, 33(3), $318-343$.

Koshy, S. (1998). Category Crisis : South Asian Americans and Questions of Race and Ethnicity1.

Kraidy, M. (2005). Hybridity, or the cultural logic of globalization. Philadelphia: Temple University Press.

Krishnan, R. (2008). Imaginary Geographies: the making of South in contemporary Tamil cinema. In Tamil Cinema: the cultural politics of India's other film industry (pp. 139-154). New York: Routledge.

Kulke, H., \& Rothermund, D. (2016). A history of India. Routledge.

Kurien, P. A. (2005). Being Young, Brown, and Hindu: The identity struggles of secondgeneration Indian Americans. Journal of Contemporary Ethnography, 34(4), 434-469. 
https://doi.org/10.1177/0891241605275575

Lal, B. (2006). The Encyclopedia of the Indian Diaspora. (B. Lal, Ed.). Singapore: Editions Didier Millet.

Lee, D. L., \& Ahn, S. (2011). Racial Discrimination and Asian Mental Health: A Meta-Analysis. The Counseling Psychologist, 39(3), 463-489. https://doi.org/10.1177/0011000010381791

Lewis, Martin W. and Wigen, K. E. (1997). The Myth of Continents A Critique of Metageography. Berkeley: University of California Press. Retrieved from http://www.ucpress.edu/book.php?isbn=9780520207431

Longstreth, R. (2008). Introduction: The Challenges of Cultural Landscape for Preservation. In R. Longstreth (Ed.), Cultural Landscapes (p. 218). Minneapolis: University of Minnesota Press.

Loomba, A. (1998). Colonialism/Postcolonialism (2nd ed.). New York: Routledge.

Lorimer, H. (2005). Cultural geography: the busyness of being "more-than-representational." Progress in Human Geography, 1, 83-94. https://doi.org/10.1191/0309132505ph531pr

Lorimer, H. (2008). Cultural geography: non-representational conditions and concerns. Progress in Human Geography, 32(4), 551-559. https://doi.org/10.1177/0309132507086882

Mackinnon, D. (2011). Progress in Human Geography Reconstructing scale : Towards a new scalar politics. https://doi.org/10.1177/0309132510367841

Manuel, I. (1997). Literary Theories In Tamil: With Special Reference To Tolkäppiyam. Pondicherry: Pondicherry Institute Of Linguistics and Culture.

Marston, S. A. (2000). The social construction of scale. Progress in Human Geography, 24(2), $219-242$.

Marston, S. A., Woodward, K., \& Jones, J. P. (2009). Scale. In D. Gregory, R. Johnston, G. 
Pratt, M. J. Watts, \& S. Whatmore (Eds.), The Dictionary of Human Geography (5th ed., pp. 664-666). Malden, MA: Wiley Blackwell.

Massey, D. S., \& Pren, K. A. (2012). Unintended consequences of US immigration policy:

Explaining the post-1965 surge from Latin America. Population and Development Review, $38(1), 1-29$.

Mavroudi, E. (2007). Diaspora as Process: (De)Constructing Boundaries. Geography Compass, 1, 467-479. https://doi.org/10.1111/j.1749-8198.2007.00033.x

Melamedoff, M. (2017). The Problem with Apu. USA: truTV.

Miller, N. P. (2008). US National Parks and management of park soundscapes: A review. Applied Acoustics, 69(2), 77-92. https://doi.org/10.1016/j.apacoust.2007.04.008

Mishra, V. (1996a). The Diasporic Imaginary: Theorizing the Indian Diaspora. Textual Practice, $10,421-447$.

Mishra, V. (1996b). The diasporic imaginary: Theorizing the Indian diaspora*. Textual Practice, 10(3), 421-447. https://doi.org/10.1080/09502369608582254

Modi, R. (2018). Communities Under Fire. SAALT.

Morris, A. (2017). W. E. B. Du Bois at the center: from science, civil rights movement, to Black Lives Matter. British Journal of Sociology, 68(1), 3-16. https://doi.org/10.1111/14684446.12241

Morrison, T. (2016). Making America White Again. The New Yorker.

Mountz, A. (2010). Seeking Asylum (NED-New). University of Minnesota Press. Retrieved from http://www.jstor.org/stable/10.5749/j.ctttv40b

Mountz, A., Coddington, K., Catania, R. T., \& Loyd, J. M. (2013). Conceptualizing detention: Mobility, containment, bordering, and exclusion. Progress in Human Geography, 37(4), 
522-541. https://doi.org/10.1177/0309132512460903

Myers, G. (2010). Representing the Other: Negotiating the Personal and the Political. In D. DeLyser, S. Herbert, S. Aitken, M. Crang, \& L. McDowell (Eds.), Sage Handbook of Qualitative Geography. London: SAGE.

Naber, N. C. (2012). Arab America: Gender, cultural politics, and activism. New York and London: NYU Press.

Nash, C. (2000). Progress reports, Performativity in practice: some recent work in cultural geography. Progress in Human Geography, 24(4), 653-664.

https://doi.org/10.1191/030913200701540654

Nash, C. (2002). Cultural geography: postcolonial cultural geographies. Progress in Human Geography, 26(2), 219-230. https://doi.org/10.1191/0309132502ph365pr

Ngai, M. M. (2004). Impossible subjects : illegal aliens and the making of modern America. Politics and society in twentieth-century America; Politics and society in twentieth-century America. TA -. Princeton, N.J. : Princeton University Press.

Nicley, E. P. (2009). Placing blame or blaming place? Embodiment, place and materiality in critical geopolitic. Political Geography, 28(1), 19-22.

https://doi.org/10.1016/J.POLGEO.2009.01.006

NJTS. (2017). New Jersey Tamil Sangam. Retrieved September 7, 2017, from http://njtamilsangam.net/web/

Paasi, A. (2003). Region and place: regional identity in question. Progress in Human Geography, 27(4), 475-485. https://doi.org/10.1191/0309132503ph439pr

Papastergiadis, N. (2015). Tracing Hybridity in Theory. In P. Werbner \& T. Modood (Eds.), Debating Cultural Hybridity: Multicultural Identities and the Politics of Anti-Racism (New, 
pp. 257-282). London: Zed Books.

Passel, J. S., \& Cohn, D. (2016). Overall Number of U.S. Unauthorized Immigrants Holds Steady Since 2009. Washington DC. Retrieved from http://www.pewhispanic.org/2016/09/20/overall-number-of-u-s-unauthorized-immigrantsholds-steady-since-2009/

Passel, J. S., \& Cohn, D. (2017). As Mexican share declined, U.S. unauthorized immigrant population fell in 2015 below recession level. Washington DC.

Peters, J. W. (2016, October 14). Among Donald Trump's Biggest U.S. Fans: Hindu Nationalists. The New York Times. Retrieved from https://www.nytimes.com/2016/10/15/us/politics/indian-americans-trump.html Pew. (2015). What Census Calls Us: A Historical Timeline. Washington DC.

Phillips, A. (2017, January 10). 10 things to know about Sen. Jeff Sessions, Donald Trump's pick for attorney general. The Washington Post. Retrieved from https://www.washingtonpost.com/news/the-fix/wp/2016/11/18/10-things-to-know-aboutsen-jeff-sessions-donald-trumps-pick-for-attorney-general/?utm_term=.cc5c806942f9

Pickles, J. (2009). Phenomenology. In D. Gregory, R. Johnston, G. Pratt, M. J. Watts, \& S. Whatmore (Eds.), The Dictionary of Human Geography. Malden, MA: Wiley Blackwell. Pijanowski, B. C., Villanueva-Rivera, L. J., Dumyahn, S. L., Farina, A., Krause, B. L., Napoletano, B. M., ... Pieretti, N. (2011). Soundscape Ecology: The Science of Sound in the Landscape. BioScience, 61(3), 203-216. https://doi.org/10.1525/bio.2011.61.3.6

Pile, S. (2010). Emotions and affect in recent human geography. Transactions of the Institute of British Geographers, 35(1), 5-20. https://doi.org/10.1111/j.1475-5661.2009.00368.x PRC. (2014). More hate crimes motivated by victims' ethnicity. Washington DC. Retrieved from 
http://www.pewresearch.org/fact-tank/2014/02/21/more-hate-crimes-motivated-by-victimsethnicity/

PTI. (2017, March 7). This Creepy Website In Ohio Stalks Indians Hanging Out At Parks, Accuses Them Of "Stealing Jobs" From Americans. Huffington Post India, p. 1. Retrieved from https://www.huffingtonpost.in/2017/03/06/this-creepy-website-in-ohio-stalks-indianshanging-out-at-parks_a_21874871/?utm_hp_ref=in-homepage

Rabaka, R. (n.d.). Forms of Fanonism : Frantz Fanon's critical theory and the dialectics of decolonization TT -. Lanham, Md. : Lexington Books,. Retrieved from https://www.dawsonera.com/guard/protected/dawson.jsp?name=https://passport01.leeds.ac. uk/idp/shibboleth\&dest=http://www.dawsonera.com/depp/reader/protected/external/Abstrac tView/S9780739140352

Radcliffe, S. A. (2017). Geography and indigeneity I : Indigeneity, coloniality and knowledge, 41(2), 220-229. https://doi.org/10.1177/0309132515612952

Radhakrishnan, R. (2003). Ethnicity in the Age of Diaspora. In Theorizing Diaspora: A Reader (pp. 119-132). Malden, MA: Blackwell Publishing Ltd.

Raimbault, M., \& Dubois, D. (2005). Urban soundscapes: Experiences and knowledge. Cities, 22(5), 339-350. https://doi.org/10.1016/j.cities.2005.05.003

Ramanujan, A. K. (1967). The Interior Landscape: Love Poems From A Classical Tamil Anthology. Bloomington: Indiana University Press.

Ramaswamy, S. (1997). Passions of the tongue: language devotion in Tamil India, 1891-1970. Berkeley: University of California Press.

Ramaswamy, S. (2009). The goddess and the nation: Mapping mother India. Duke University Press. 
Rathinasabapathy, V. (2008). From Language To The Life-World: Perspectives Of The Tolkāppiyam. In R. Balasubramanian (Ed.), The Life-World Of The Tamils: Past and Present (pp. 139-173). New Delhi: Project Of History Of Indian Science, Philosophy and Culture.

Ravi, S. (2008). Tamil identity and diasporic desire in a Kollywoood comedy: Nala Damayanti (2003). South Asian Popular Culture, 6(1), 45-56.

Ray, J. K., \& Mishra, B. K. (2009). Interpreting the Indian diaspora: Lessons from history and contemporary politics. Centre for Studies in Civilizations for the Project of History of Indian Science, Philosophy and Culture.

Revill, G. (2016). How is space made in sound? Spatial mediation, critical phenomenology and the political agency of sound. Progress in Human Geography, 40(2), 1-17. https://doi.org/10.1177/0309132515572271

Riesenweber, J. (2008). Landscape Preservation and Cultural Geography. In R. Longstreth (Ed.), Cultural Landscapes (p. 218). Minneapolis: University of Minnesota Press.

Rose, G. (2003). On the Need to Ask How, Exactly, Is Geography "Visual”? Antipode, 35(2), 212-221. https://doi.org/10.1111/1467-8330.00317

Rose, G. (2016). Visual Methodologies (4th ed.). London: SAGE.

SAALT. (2015). A Demographic Snapshot of South Asians in the United States, (December), 2. Retrieved from http://saalt.org/wp-content/uploads/2016/01/Demographic-Snapshotupdated_Dec-2015.pdf

Safran, W., Kumar Sahoo, A., \& Lal, B. V. (2008). Indian Diaspora in Transnational Contexts: Introduction. Journal of Intercultural Studies, 29(1), 1-5. https://doi.org/10.1080/07256860701759907 
Sahoo, A. K. (2006). Issues of Identity in the Indian Diaspora: A Transnational Perspective. Perspectives on Global Development and Technology, 5(1), 81-98. https://doi.org/10.1163/156915006777354482

Said, E. (1978). Orientalism. New York: Pantheon Books: Random House.

Said, E. (1993). Culture and Imperialism. New York: Vintage Books: Random House.

Said, E. (1997). Covering Islam: How the Media and the Experts Determine How We See the Rest of the World. New York: Vintage Books: Random House.

Saldanha, A. (2005). Trance and visibility at dawn: racial dynamics in Goa's rave scene. Social \& Cultural Geography, 6(5), 707-721. https://doi.org/10.1080/14649360500258328

Saldanha, A. (2007). Psychedelic white: Goa trance and the viscosity of race. Minneapolis: University of Minnesota Press.

Saldanha, A. (2010). Politics and Difference. In B. Anderson \& P. Harrison (Eds.), TakingPlace: Non-Representational Theories and Geography (pp. 283-302). Farnham: Ashgate. Retrieved from http://www.amazon.com/Taking-Place-Non-Representational-GeographyBen-Anderson/dp/0754672794

Samuels, D. W., Meintjes, L., Ochoa, A. M., \& Porcello, T. (2010). Soundscapes: Toward a Sounded Anthropology. Annual Review of Anthropology, 39(1), 329-345. https://doi.org/10.1146/annurev-anthro-022510-132230

Sarah Neal. (2002). Rural landscapes, representations and racism: examing multicultural citizenship and policy-making in the English countryside. Ethnic and Racial Studies, 25(3), 442-461. https://doi.org/10.1080/01419870020036701

Sastri, K. A. N. (1975). A history of South India from prehistoric times to the fall of Vijayanagar (3rd ed.). London: Indian Branch, Oxford University Press. 
Schafer, R. M. (1994). The Soundscape: Our Sonic Environment and The Tuning of the World. Vermont: Destiny Books.

Schrank, B. (2007). “Cutting Off Your Nose to Spite Your Race”: Jewish Stereotypes, Media Images, Cultural Hybridity. Shofar: An Interdisciplinary Journal of Jewish Studies, 25(4), $18-42$.

Schwartzberg, J. E., \& S.G. Bajpai. (1992). A Historical Atlas of South Asia (2nd ed.). New York: Oxford University Press.

Segrott, J. (2001). Language, geography and identity: the case of the Welsh in London. Social \& Cultural Geography, 2(3), 281-296. https://doi.org/10.1080/14649360122936

Selby, M. A., \& Peterson, I. V. (2008). Tamil Geographies Cultural Constructions of Space and Place in South India. Albany: University of New York Press.

Seth, V. (2010). Europe's Indians: producing racial difference, 1500-1900. Durham: Duke University Press.

Sharma, R., \& Annamalai, E. (2003). Indian diaspora : in search of identity. In Languages., Central Institute of Indian (Mauritius), Mahatma Gandhi Institute. Mysore: Central Institute of Indian Languages.

Sharma, S. (2006). Asian Sounds. In A postcolonial people: South Asians in Britain.

Sharp, J. (2009). Geography and gender: what belongs to feminist geography? Emotion, power and change. Progress in Human Geography, 33(1), 74-80. https://doi.org/10.1177/0309132508090440

Sharp, J. (2011). A subaltern critical geopolitics of the war on terror: Postcolonial security in Tanzania. Geoforum, 42(3), 297-305. https://doi.org/10.1016/j.geoforum.2011.04.005 Sharp, J., Briggs, J., Sharp, J., \& Briggs, J. (2006). Postcolonialism and Development: New 
Dialogues? The Geographical Journal, 172(1), 6-9.

Sidaway, J. D. (2000). Postcolonial geographies: an exploratory essay. Progress in Human Geography, 24(4), 591-612. https://doi.org/10.1191/030913200100189120

Sidaway, J. D. (2012). Geography, Globalization, and the Problematic of Area Studies. Annals of the Association of American Geographers, 5608(April), 120411125724008. https://doi.org/10.1080/00045608.2012.660397

Silvey, R. (2014). Gender and mobility Critical ethnographies of migration in Indonesia. In Cultural Geography in Practice (pp. 105-119). Routledge.

Simonsen, K. (2010). Encountering O / other Bodies : Practice, Emotion and Ethics. In B. Anderson \& P. Harrison (Eds.), Taking-Place: Non-representational Theories and Geography (pp. 221-2139). Farnham: Ashgate.

Skop, E. (2012). The Immigration and Settlement of Asian Indians in Phoenix, Arizona, 19652011: Ethnic Pride Vs. Racial Discrimination in the Suburbs. Edwin Mellen Press.

Skop, E., \& Adams, P. C. (2009). Creating and inhabiting virtual places: Indian immigrants in cyberspace. National Identities, 11(2), 127-147.

Skop, E., \& Li, W. (2005). Asians in America's suburbs: Patterns and consequences of settlement. Geographical Review, 95(2), 167-188.

Smith, N. (1996). Spaces of Vulnerability: The space of flows and the politics of scale. Critique of Anthropology, 16(1), 63-77. https://doi.org/10.1177/0308275X9601600107

Smith, S. (2000). Performing the (sound) world. Environment and Planning D: Society and Space, 18(5), 615-637. https://doi.org/10.1068/d225t

Smith, S. (2011). "She says herself, “I have no future "': love, fate and territory in Leh District , India. Gender, Place \& Culture, 18(February 2015), 455-476. 
https://doi.org/10.1080/0966369X.2011.583344

Smith, S. J. (1994). Soundscape. Area, 26(3), 232-240.

Southworth, M. (1969). The Sonic Environment of Cities. Environment and Behavior, 1(1), 4971.

Sparke, M. (1998). A Map that Roared and an Original Atlas: Canada, Cartography, and the Narration of Nation. Annals of the Association of American Geographers, 88(3), 463-495. https://doi.org/10.1017/CBO9781107415324.004

Spivak, G. C. (1988). Can the Subaltern Speak? In C. Nelson \& L. Grossberg (Eds.), Marxism and the Interpretation of Culture (pp. 271-317). University of Illinois Press.

Sridaran, L., Raghunathan, S., \& Trivedi, V. (2017). Power, Pain, Potential: South Asians at the Forefront of Growth and Hate in the 2016 Election Cycle. Takoma Park.

Srivastava, P. (2013, August 24). Why Bollywood still can't get over cultural stereotypes. Daily Mail, p. 1. Retrieved from http://www.dailymail.co.uk/indiahome/indianews/article2401067/WEEKEND-ENTERTAINMENT-Why-Bollywood-culture-stereotypes.html

Steinberg, P. E., Page, S., Dittmer, J., Gökariksel, B., Smith, S., Ingram, A., \& Koch, N. (2018).

Reassessing the Trump presidency, one year on. Political Geography, 62, 207-215. https://doi.org/10.1016/j.polgeo.2017.10.010

Sthapati, V. G. (2008). Sthāpatya-Veda and traditional building architecture. In R. Balasubramanian (Ed.), The life-world of the Tamils: past and present (pp. 777-883). New Delhi: Project of History of Indian Science, Philosophy and Culture.

Tacchi, J. A., Slater, D., \& Hearn, G. (2003). Ethnographic action research: A user's handbook. Talbot, M. (2015). The Story of a Hate Crime. The New Yorker, 14. Retrieved from https://www.newyorker.com/magazine/2015/06/22/the-story-of-a-hate-crime 
Thien, D. (2005). After or beyond feeling? A consideration of affect and emotion in geography. Area, 37(4), 450-454. https://doi.org/10.1111/j.1475-4762.2005.00643a.x

Thrift, N. (2008). Non-Representational Theory. New York: Routledge.

Tolia-Kelly, D. P. (2006). Affect - An ethnocentric encounter? Exploring the "universalist" imperative of emotional/affectual geographies. Area, 38, 213-217. https://doi.org/10.1111/j.1475-4762.2006.00682.x

Tölölyan, K. (1996). Rethinking Diaspora(s): Stateless Power in the Transnational Moment. Diaspora: A Journal of Transnational Studies, 5(1), 3-36. https://doi.org/10.1353/dsp.1996.0000

Tummala-Narra, P., Alegria, M., \& Chen, C. N. (2012). Perceived discrimination, acculturative stress, and depression among South Asians: Mixed findings. Asian American Journal of Psychology, 3(1), 3-16. https://doi.org/10.1037/a0024661

UN. (2015). International Migration Report. New York. Retrieved from http://www.un.org/en/development/desa/population/migration/publications/migrationreport/ docs/MigrationReport2015_Highlights.pdf

USCIS. (2017a). Combating Fraud and Abuse in the H-1B Visa Program. Retrieved October 8, 2017, from https://www.uscis.gov/working-united-states/temporary-workers/h-1b-specialtyoccupations-and-fashion-models/combating-fraud-and-abuse-h-1b-visa-program

USCIS. (2017b). Putting American Workers First: USCIS Announces Further Measures to Detect H-1B Visa Fraud and Abuse. Retrieved April 17, 2017, from https://www.uscis.gov/news/news-releases/putting-american-workers-first-uscis-announcesfurther-measures-detect-h-1b-visa-fraud-and-abuse

Velayutham, S. (2008a). Introduction. In S. Velayutham (Ed.), Tamil Cinema: the cultural 
politics of India's other film industry.

Velayutham, S. (2008b). The diaspora and the global circulation of Tamil cinema. In Tamil Cinema: the cultural politics of India's other film industry (pp. 172-188). New York: Routledge.

Veracini, L. (2013). 'Settler colonialism': Career of a concept. The Journal of Imperial and Commonwealth History, 41(2), 313-333.

Vertovec, S. (1999). Three Meanings of "Diaspora," Exemplified among South Asian Religions. Diaspora: A Journal of Transnational Studies, 6(3), 277-299. https://doi.org/10.1353/dsp.1997.0010

Viswanathan, T., \& Allen, M. H. (2004). Music in South India: The Karnatak concert tradition and beyond. New York: Oxford University Press.

Voigt-Graf, C. (2004). Towards a geography of transnational spaces: Indian transnational communities in Australia. Global Networks, 4(1), 25-49. https://doi.org/10.1111/j.14710374.2004.00079.x

Waterman, S. (2006). Geography and music: Some introductory remarks. GeoJournal, 65(1-2), 1-2. https://doi.org/10.1007/s10708-006-7047-2

Winders, J. (2005). Changing politics of race and region: Latino migration to the US South. Progress in Human Geography, 29(6), 683-699. https://doi.org/10.1191/0309132505ph577oa

Winders, J. (2007). Bringing Back the (B) order: Post 9/11 Politics of Immigration, Borders, and Belonging in the Contemporary US South. Antipode, 39(5), 920-942. https://doi.org/10.1111/j.1467-8330.2007.00563.x

Winders, J. (2008). An "Incomplete" Picture? Race, Latino Migration, and Urban Politics in 
Nashville, Tennessee. Urban Geography, 29(3), 246-263. https://doi.org/10.2747/02723638.29.3.246

Wolf, R., \& Sherinian, Z. (2000). No Title. In B. Nettl \& A. Arnold (Eds.), The Garland encyclopedia of world music: South Asia, the Indian subcontinent (pp. 903-929). New York: Garland.

Wylie, J. (2005). A single day's walking: Narrating self and landscape on the South West Coast Path. Transactions of the Institute of British Geographers, 30(2), 234-247. https://doi.org/10.1111/j.1475-5661.2005.00163.x

Wylie, J. (2010). Non-Representational Subjects? In B. Anderson \& P. Harrison (Eds.), TakingPlace: Non-Representational Theories and Geography (pp. 99-114). Farnham: Ashgate.

Yoo, H. C., \& Lee, R. M. (2005). Ethnic identity and approach-type coping as moderators of the racial discrimination/well-being relation in Asian Americans. Journal of Counseling Psychology, 52(4), 497-506. https://doi.org/10.1037/0022-0167.52.4.497

Young, R. J. C. (1995). colonial desire: Hybridity in Theory, Culture and Race. London: Routledge.

Zeitlyn, B. (2013). Making sense of the smell of Bangladesh. Childhood, 21(2), 175-189. https://doi.org/10.1177/0907568213488965

Zong, J., \& Batalova, J. (2015). Frequently requested statistics on immigrants and immigration in the United States. Migration Policy Institute, 26, 1-18. 


\section{Appendix A}

\section{Interview Guide}

*Note - this was a rough interview guide. I asked questions related to this but deviated based on responses and the amount of time for participant responses.

1. Introductions

2. Present Cover Letter and explain the research

3. Ask if they have questions

4. Ask for Verbal Consent (and consent to record)

a. Interviews are totally anonymous (unless you want me to use your name)

b. You do not have be recorded - it is for my benefit - so I don't misinterpret

5. Can you tell me a little bit about your Tamil background?

a. What your Generation?

b. How long have you/family been in the US?

c. Where are you from?

d. Where are your parents from?

6. Define identity as a social scientist. identity is the qualities, beliefs, personality, looks and/or expressions that make a person (self-identity) or group (particular social category or social group). Things like race, class, sexual orientation, gender, education, origin, ethnicity, etc.

7. Can you write down some words that come to mind to describe your identity? Include things that you feel are important to your identity -categories that might be social, cultural, economic, might pertain to work, religions, home, age, gender, sexuality. For the sake of this project I am going to focus on the aspects that have to do with places.

a. In your opinion, what does it mean to be (point to identity) and what qualities are associated with this?

b. How does being Tamil affect your sense of identity of being Indian?

8. What sounds (define) are associated with being ?

9. Can you think of some sounds that you might consider part of your identity, i.e. language, music, environmental sounds (associated with these national identities?).

a. How do these sounds make you feel?

b. Are there sounds songs/languages/accents that invoke memories?

c. Or make you feel Tamil? 
d. If you want to feel more Tamil is there something you listen to?

10. Another goal of my project is to understand how some Americans feel marginalized with these very complicated identities) Have you ever felt marginalized (biases, discrimination) based on your identities?

a. Does sound work as a marker of identity that they feel discriminated against?

b. Do you intentionally or sub-consciously speak differently or listen to different music to disguise or accentuate certain identities?

c. Demographic questions: What is your education level?

d. Would you like to tell me your age?

11. Ask them if they want to participate in the follow up AudioVoice/Photovoice where they spend 2-3 weeks recording sounds or images that are meaningful to them or their identities. Or recording instances where they may have felt marginalized.

12. How would you rate to importance of sound to your everyday identities on a scale of 1-5 ( 5 being the highest and 1 being the lowest)?

13. Thank them and tell them to contact if they have any questions. 


\section{Appendix B}

\section{Initial Codes}

*These are the basic coding categories I developed in NVivo. I looked to see where they intersected - i.e. accent's intersection with discrimination. These reflect the very beginning of my analysis. These codes developed into broader themes within the dissertation.

- Descriptors of Identity

$\begin{array}{ll}\circ & \text { active reference to sexuality } \\ \circ & \text { Clothes } \\ \circ & \text { Diversity } \\ \circ & \text { Economic } \\ \circ & \text { Emotion } \\ \circ & \text { Family } \\ \circ & \text { Food } \\ \circ & \text { Generation } \\ \bigcirc & \text { Literature } \\ \bigcirc & \text { Media } \\ \bigcirc & \text { Memory } \\ \bigcirc & \text { Place } \\ \bigcirc & \text { Religion } \\ \bigcirc & \text { Self } \\ \circ & \text { Smells }\end{array}$

- Identity

- American

- Dravidian

O Indian

- Indian American

- Other Identity

- Malaysia

- Pakistan

- South Indian

- Tamil identity

- Brown

○ Desi

- Village

- Region (N/S TN)

○ City

- County/district

- Home

○ US state
- Global +

- South Asian

- Tensions between identities

- Indian/American

- Indian/Tamil

o Tamil/etc.

- Power Relations

- Caste

- Discrimination

- Othering

- Hate Crimes

- Threats

- Orientalism

- Gender

o Hegemony

- Indian racism

- Within communities

- Outside of communities

- Pronunciation-Name

- Skin-Looks

- Trump

- 9/11

- Sound

- Accent

- Environmental Sounds

- lack of sound

$\bigcirc$ Language

- Music 


\title{
Appendix C
}

\author{
Recruitment Letter
}

\section{WestVirginiaUniversity.}

This is a request your participation in a research project to assess how music, accent, and language affect identity. This project is being conducted by Christabel Devadoss, PhD Candidate in the department of Geology and Geography at WVU with supervision of Dr. Karen Culcasi, an associate professor in the department of Geology and Geography, for a Doctoral Degree in Geography. I am looking for participants 18 years of age or older, have the ability to speak English, and identify as Tamil or a Tamilspeaking member of the Asian Indian community to answer some questions related to these issues. Participation in this project is greatly appreciated and will be completely anonymous. It will take approximately 30 minutes -1 hour to complete the interview. If you are interested, please contact me at cadevadoss@mix.wvu.edu or 440-994-9102.

Phone: 304-293-7073 Fax: 304-293-3098

http://oric.research.wvu.edu
Chestnut Ridge Research Building

886 Chestnut Ridge Road

PO Box 6845

Morgantown, WV 26506-6845 
